BOSTON UNIVERSITY

GRADUATE SCHOOL OF ART'S AND SCIENCES

Disscrtation

\title{
A MEASUREMENT OF THE MASS OF THE TOP QUARK IN THE DI-LEPTON CHANNELS USING THE DØ DETECTOR AT FERMILAB
}

by

\section{SAROSH NOSHIR FATAKIA}

B.Sc., University of Calcutta, 1994

M.Sc., University of Calcutta, 1996

M.S., Syracusc University, 1999

Submitted in partial fulfillment of the

requirements lor the degree of

Doctor of Philosophy

2005 
Approved by

First Reader

Llrich Heintz, Ph.D.

Associate Professor of Physics

Sccond Reader

J. Scott Whitaker, Ph.D.

Professor of Physics 
(c) Copyright by SAR0SH NOSHIR FATAKIA

2005 


\section{Dedication}

To those who have nurtured me and inspire me:

my Mother, $\mathcal{F}$ ather, and Sister,

Master-Moshai,

Bapuji, Rustomji, Aderbad,

Desaiji, Ratanshah, Rustomji,

$\& \mathcal{T}$ ina Aunly.

To those who guide me:

$\mathcal{B}$ apaiji, $\mathcal{M}$ amaijis, $\mathcal{B}$ apawaji, $\mathcal{M}$ amawaji, $\mathcal{F}$ reny $\mathcal{M}$ asi, $\mathcal{H}$ omi $\mathcal{K}$ aka, Homi $\mathcal{F}$ ua, Dali Kaka. 


\section{Acknowledgments}

It has been a wonderful experience to be a part of the Boston University community as well as the Fermilab community. I would like to thank both communitics which have nurtured me and helped me become a better and brighter person. However, I want to thank everyone individually who has helped me. Omission of any individual acknowledgment is purcly accidental and unintentional.

First, I would like to express my sincere thanks and gratitude to my academic adviser and mentor, Professor Ulrich Heintz for his invaluable guidance and support. I have been particularly fortunate to be Ulrich's first graduate student and absorb and experience his knowledge and expertise in this field. It has been a pleasure.

I would like to express my heartfelt thanks to the Professors in my dissertation committee as well. I am grateful to Professor Ileintz, Professor Whitaker, Professor Chivukula, Professor Erramilli, Professor Bansil and Professor Brower for valuable suggestions and help during ny Ph.D and also during the formulation of my disscrtation. Special thanks to Prolessor Heintz, Dean Whitaker as well as Prolessor Brower, for patiently and carefully correcting the same.

I would also like to thank Tom Ferbel, John Butler, Meenakshi Narain, Marek, Aurclio, Lars, Ia, Chris, Rcgina, Erich, Ivor, Ela, Cccilia, Arnulf, Regina, Suyong, Marco, Avto, Lisa, Christophe Clement, Christophe Royon, Jean-Laurcnt Agram, Christian Schmitt, Jodi, Jason, Alcx Zabi, Lorcnzo, Aricl, Amitabha, Kevin and each and every one in our collaboration. In particular, for the assistance with event selection, I would like to thank Alan, Stefan, Jeff, Joe, Ashish, Kirti, Sara, Danicl and Prolay. Thanks to Abid, Alexander Kupco, Alcx Melnitchouk, Dennis, Greg, Junjie, Markus Klute, Nirmalya and Supriya for providing some plots for the dissertation. 
Spccial thanks to Rene Brun, Philippe Canal, Cclina Paul, Guoan Hu, Mirtha Cabello, Martha Khan and Brendan for their expertise and technical support. Thanks to the folks at Fermilab's Bcams Division, and the Housing Office: Jackic, Cheryl, Linda, Tom and many more.

I would like to thank all at my Alma Mater Syracuse University and at Saha Insititute of Nuclcar Physics. I am gratcful for the knowledge and expericnce I received from my Professors and friends there. I am indebted to Bal, Joe, Carl, Eric, Ralacl, Cristina, Don, Shcldon, Tomasz, Marina and Sacha. Special thanks to Jianchun and Gobinda.

I would like to take this opportunity to thank my friends and well wishers. I am especially indebted to Himel, Minu Uncle, Roshan, Rashna, Benaifer and Mahazabeen. I would like to thank my friends from all over the globe. The twinkle in their cyes and their smile always light my life: Avan and Zarin, fricnds Natcrcia, Rashna, Valentina, Kamalrukh, Renata, Ana, Helena, Amber, Lydia, Emily, Tamsin, Miruna, Cristina, Benailer, Binailcr, Mahazabecn, Rushna, Decnaz, Dilshad and Sanghamitra. Thanks to Arnavaz Aunty and Marazban, Mehroo Aunty, Gul Aunty, Shahrokh Uncle, Dolly Aunty, Jangoo Uncle, my Masi ji, Masajis, Kakijis and Kakaji, Fuijis, Fuaji, Arunda and Boudi, Ayan, my Professors and my buddics during M.S. and B.S. at Calcutta. Thank you David, Hiroki, Shantenu, Farshid, Animesh, Bani, Avdhesh, Jyothsna, Piyali, Scrge, Anatoly, Jiri, Rob, Ricardo, Jorge, Juan, Pcdro, Eduard, Sameet, Pradeep, Rostem, Edul, Yezad, Ariz, Urvaksh, Jamshed, Pedrame, Hoshedar, Kersi, Khurshed, Rohinton, Navaz, Hormuzd, Viraf, Zubin and Keki.

The discipline and education I received from primary school to high school has helped shape my life. To all my family, Master-Moshai and his family, my teachers and fricnds at Calcutta Boys' School, thank you very much. 


\title{
A MEASUREMENT OF THE MASS OF THE TOP QUARK IN THE DI-LEPTON CHANNELS USING THE DØ DETECTOR AT FERMILAB
}

\author{
(Order No.
}

\section{SAROSH NOSHIR FATAKIA}

Boston University Graduate School of Arts and Scicnces, 2005

Major Professor: Ulrich Heintz, Associate Professor of Physics

\begin{abstract}
$\Lambda \mathrm{BST} \mathrm{T}^{\prime} \Lambda \mathrm{Cl}^{\prime}$
This dissertation describes a measurement of the mass of the top quark using events consistent with the hypothesis $t \bar{t} \rightarrow b W^{+} \bar{b} W^{-} \rightarrow b l^{+} \nu \quad \bar{b} l^{-} \bar{\nu}$, where $(l=e$, $\mu$ ). The events are obtained from nearly $230 \mathrm{pb}^{-1}$ of $p \bar{p}$ collision data collected by the Dø experiment between 2002 and 2004 during Run II. In this decay channel two neutrinos remain undetected. Extraction of the mass of the top quark by kincmatic reconstruction is not possible because the event is under-constrained. Therefore, a dynamical likelihood method is developed to obtain the mass of the top quark. The mass of top quark obtained from the candidate events selected in the di-clectron channel and the $e \mu$ channel is:
\end{abstract}

$$
154.1_{-12.8}^{+14.2} \text { (stat.) } \pm 6.6 \text { (syst.) GoV. }
$$




\section{Contents}

Acknowledgments $\quad$ v

Abstract vii

Table of Contents viii

List of Tables $\quad$ xiv

List of Figures $\quad$ xviii

List of Abbreviations $\quad$ xxvi

1 Introduction 1

1.1 The fronticr of particle physics . . . . . . . . . . . . 2

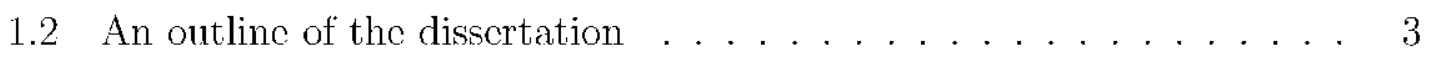

1.3 Conventions and terminology ................. 4

$\begin{array}{lll}2 & \text { Theoretical Context } & 6\end{array}$

2.1 Synopsis of the SM of particle physics . . . . . . . . . . . . 6

2.1.1 The free parameters in the SM . . . . . . . . . . 9

2.1.2 Fvidence for the existence of the top quark . . . . . . . . . 10 
2.2 Some lindamental propertics of the top quark . . . . . . . . . . . . 11

2.3 Significance of the top quark mass . . . . . . . . . . . . . 15

2.3.1 An indirect consequence of the top mass:

radiative corrections and indirect constraints ........ 15

2.4 Top quark production in proton anti-proton collisions,

and thcir subscquent decay modes. . . . . . . . . . . 17

3 Experimental Context $\quad 22$

3.1 SM measurements in the $\mathrm{EW}$ sector . . . . . . . . . . . . . . 22

3.1.1 The mass of the top quark . . . . . . . . . . . . . 22

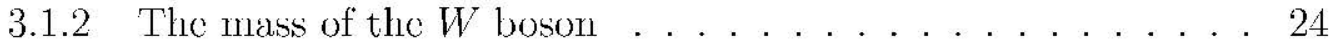

3.2 SM analysis of the free parameters . . . . . . . . . . . . 27

3.2.1 The SM predictions . . . . . . . . . . . . . . 29

4 The Expcrimental Sctup $\quad 32$

4.1 The Fermilab Tevatron accelerator . . . . . . . . . . . . . . 32

1.1.1 Gcneration and accelcration of protons and anti-protons . . . 33

4.2 The I) $\varnothing$ detector $\ldots \ldots \ldots \ldots$. . . . . . . . . . . . . . . . . . . .

4.2.1 The DØ detector coordinate system . . . . . . . . . . 38

4.2 .2 The detector sub-systems . . . . . . . . . . . . . 40

5 Simulations $\quad 54$

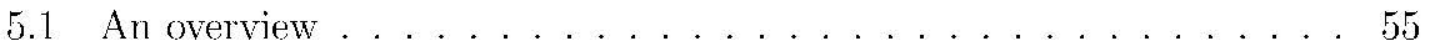

5.2 Simulation of the physics processes . . . . . . . . . . . . . 61

5.3 Simulation of the $\mathrm{D} \emptyset$ detector . . . . . . . . . . . . . 62

5.4 Additional corrections on simulated events . . . . . . . . . . . . 64 
6 Data Selection $\quad 65$

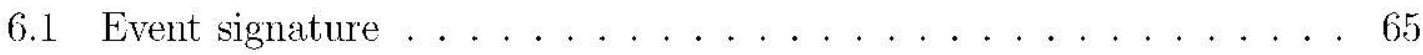

6.2 The strategy of cvent selection . . . . . . . . . . . . 68

6.3 On-line trigger selection . . . . . . . . . . . . . . 70

6.3.1 L1 EM objects, jet objects and muons . . . . . . . . . . . 71

6.3.2 L2 EM objccts, jet objects and muons . . . . . . . . . . . 72

6.3.3 L3 EM objects, jet objects and muons . . . . . . . . . . . . . 74

6.4 Ol[-line reconstruction . . . . . . . . . . . 75

6.4.1 Reconstruction of a track object . . . . . . . . . . 76

6.4 .2 Reconstruction of the primary vertex . . . . . . . . . 76

6.4.3 Reconstruction of muon objects . . . . . . . . . . 77

6.4.4 Reconstruction of electron objects . . . . . . . . . . . . 78

6.4.5 Reconstruction of jet objccts . . . . . . . . . 79

6.4.6 Corrections to off-line objects . . . . . . . . . . . 80

6.4.7 Determination of the unbalanced transverse momentum . . . . 80

6.5 Selection cuts . . . . . . . . . . . . . . . . . . 80

6.5.1 Selection cuts for track objects used in reconstructing

the primary vertex. . . . . . . . . . . . . 81

6.5.2 Selection cuts for the primary vertex identification . . . . . . 81

6.5.3 Sclection cuts for muon identification . . . . . . . . 81

6.5.4 Selection cuts for electron identification . . . . . . . . . . 83

6.5.5 Selection cuts for jet identification . . . . . . . . . . 83

6.6 Expoctod signal and background yiclds . . . . . . . . . . . . . . 84

6.7 Selected data sample . . . . . . . . . . . . . . . . 86 
7 Detector Calibration and Resolution $\quad 87$

7.1 Calibration of the electron energy scale . . . . . . . . . . . 89

7.2 Calibration of the muon momentum scale . . . . . . . . . . . . 93

7.3 Calibration of the jet energy scale . . . . . . . . . . 96

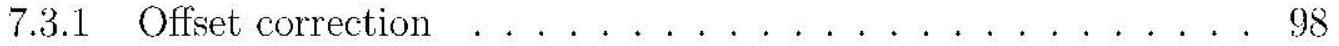

7.3 .2 Responsc correction . . . . . . . . . . . . . . 98

7.3.3 Showering correction . . . . . . . . . . . . . 99

7.1 Evaluation of the missing transverse momentum . . . . . . . . . . 102

7.5 Correcting the jet 4-vector to represent the parton 4-vector . . . . . . 103

7.6 Electron energy resolution . . . . . . . . . . . . . . . . 108

7.7 Muon momentum resolution . . . . . . . . . . . . . . . . . . 112

7.8 Jet energy resolution . . . . . . . . . . . . . . . . . . 116

7.9 Summary of the object resolutions . . . . . . . . . . . 118

8 Mass Measurement $\quad 119$

8.1 The di-lepton event topology . . . . . . . . . . . . . . . . 119

8.1.1 Constraints from the event topology . . . . . . . . . . . 121

8.2 The Mcthod of analysis . . . . . . . . . . . . . . . . 127

8.2.1 The mass analysis of an event . . . . . . . . . . . . 127

8.3 The mass analysis: the first step . . . . . . . . . . . . 130

8.3.1 The peak weight as the mass estimator . . . . . . . . . . . 131

8.4 Mass analysis using an ensomble of cvents . . . . . . . . . . 133

8.4.1 The Maximum Ijikelihood Function . . . . . . . . . . . . 133

8.4.2 Statistical uncertainty from ensemble studies . . . . . . . . 138

8.4.3 Tomplate construction . . . . . . . . . . . 110 
8.1 .4 The data enscmble . . . . . . . . . . . . . . 145

8.4.5 Construction of simulated ensembles for sell-consistency tests . . . . . . . . . . . . . . 146

8.5 Evaluation of the Maximum Iikelihood Fstimate. . . . . . . . . 147

8.5.1 The Maximum Likelihood Estimate using simulated enscmbles . . . . . . . . . . . . . 147

8.6 Self-consistency tests using fast MC . . . . . . . . . . . 150

8.7 Self-consistency tests with simulated events incorporating the full detector resolution effects . . . . . . . . 154

8.8 The Maximum Likelihood Estimate using the data ensemble . . . . 158 8.8.1 Results from the data ensembles: $e \mu$ and ee channels . . . 158

8.8.2 MLE from data ensemble with bias correction. . . . . . . . . 163

8.8 .3 A cavcat . . . . . . . . . . . . . . . . . 165

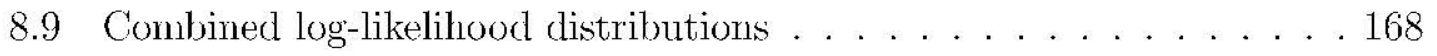

8.10 Systcmatic unccrtaintics . . . . . . . . . . . . . 169

8.10 .1 The jet energy scale $\ldots \ldots \ldots \ldots$

8.10.2 Electron energy and muon momentum scale . . . . . . . . 176

8.10 .3 Multiple parton intcractions . . . . . . . . . . . 176

8.10.4 Signal event generator . . . . . . . . . . . 176

8.10.5 Enscmblc Calibration Curve . . . . . . . . . . 177

8.10.6 Signal and background estimation . . . . . . . . . 177

8.10 .7 Miscellaneous issues . . . . . . . . . . . . . . . . . . 177

8.11 The combincd systcmatic uncertainty . . . . . . . . 178

8.12 The measured mass . . . . . . . . . . . . . . . . 180

8.13 Salient features of the mass analysis . . . . . . . . . 180 
9 Comparison With Other Measurements

9.1 Independent measurements in the di-lepton channel . . . . . . . . . 183

9.2 Independent measurements from Run II . . . . . . . . . . . . . 186

9.2.1 Recent results from the I) $\varnothing$ experiment . . . . . . . . . 186

9.2 .2 Recent results from the CDF experiment . . . . . . . . . . 188

$\begin{array}{lr}10 \text { Conclusion and future outlook } & 189\end{array}$

$\begin{array}{ll}\text { A Glossary } & 191\end{array}$

B A brief history of my efforts 193

C Interactions of final-state particles in the detector 195

D An illustration of the application of simple topological criteria towards optimizing the Monte Carlo production $\quad 197$

E Some L1 TT studies $\quad 206$

F Additional information regarding average corrections to the jet 4-vectors for representing parton 4-vectors $\quad 217$

G Additional information regarding template distributions

H Additional information regarding the Maximum Likelihood Estimates using the negative log-likelihood fits to event ensembles

I Additional information from simulated ensemble studies: the MLE and pull distributions 
J A study of the bias in ensemble calibration

K Kinematic information of candidate events

L Normalized weight distribution of candidate events

Bibliography

Curriculum Vitae 


\section{List of Tables}

2.1 Some properties of the constituent particles of the SM of particle physics. ...................... 7

2.2 Possible decay modes for the $W^{+} W^{-}$daughter pair from the $t \bar{t}$ pair. . 20

3.1 Overvicw of some electroweak parameters. . . . . . . . . 28

3.2 Global SM fits to electroweak parameters from data. . . . . . . . 29

4.1 Table of various detector sub-systems and their geometrical acceptance in pseudorapidity. . . . . . . . . . . . . . 41

6.1 Triggers applicd at the L1 stages for selecting di-lepton events. . . . . 71

6.2 Triggers applied at the Is stage for selecting di-lepton events. . . . . 72

6.3 A list of electron ID definitions used in reconstruction algorithms. . . 79

6.1 The expected signal and background yiclds, observed number of events for the di-electron channel. . . . . . . . . . . . . . . . . . . 84

6.5 The expected signal and background yiclds, and observed number of events for the di-muon channel. . . . . . . . . . . . 85

6.6 Expected background yields, expected signal yield and observed number of events for the $e \mu$ channcl. . . . . . . . . . 85 
6.7 Rum numbers and cvent numbers for the selected events in the dielectron channel. .................... 86

6.8 Run numbers and cvent numbers for the selected events in the $e \mu$

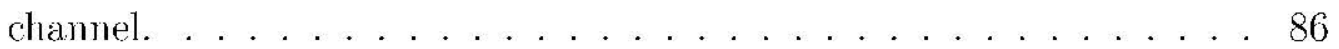

7.1 Physics processes used in the energy-momentum calibration of the

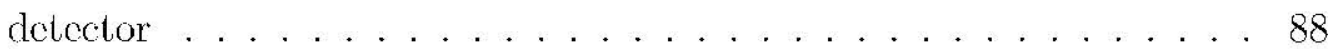

7.2 Scale and oversmearing corrections for simulated events. . . . . . . . 91

7.3 Ranges of detector $\eta$ used to obtain the jet energy scale. . . . . . . . 102

7.4 The average fraction of the jet encrgy contained in the fixed cone algorithms as a function of detector $\eta$ (from Tevatron data). . . . . . 102

7.5 The average fraction of cnergy contained in fixed cone jets as a function of detector $\eta$ (from $\mathrm{MC}$ events). . . . . . . . . . . . . 103

7.6 Coefficients for jets matched to light quarks, as a function of detector

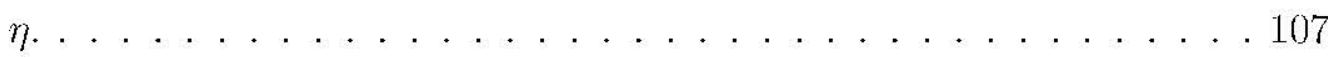

7.7 Coefficients for jets matched to $b$ quarks, as a function of detector $\eta . .107$

7.8 Coefficionts for jets without muon matched to $b$ quarks, as a function of detector $\eta$. . . . . . . . . . . . . . . . . . . . . 107

7.9 Coefficients for jets with muons matched to $b$ quarks, as a function of detector $\eta$. . . . . . . . . . . . . . . . . . . . . 107

7.10 Energy resolution parameters for the central calorimeter (CC) and end calorimcter (EC) as a function of $\eta_{\text {del }}$. . . . . . . . . . . . . . 109

7.11 The parameterization of resolution for reconstructed muons. . . . . . 118

7.12 The parameterization of resolution for reconstructed electrons. . . . . 118

7.13 The parametcrization of resolution for reconstructed jets. . . . . . 118 
8.1 The composition of templates and simulated cnsembles expressed as a percentage of the total composition. . . . . . . . . . . . 147

8.2 Results from sinmlated cnscmbles of 8 e $\mu$ cvents. . . . . . . . . . . . 119

8.3 Results from simulated ensembles of 5 di-electron events. . . . . . . . 149

8.4 Results from simulated ensembles with $8 \mathrm{e \mu}$ events. . . . . . . . . . 154

8.5 Results from simulated ensembles with 5 di-clectron cvents. . . . . . . 151

8.6 Log-likelihood versus input MC $m_{t}$ for the $e \mu$ ensemble. . . . . . . . . 158

8.7 Log-likelihood versus input MC $m_{\ell}$ for the ee ensemble. . . . . . . . . 158

8.8 Systematic uncertainties in the e $\mu$ channel. . . . . . . . . . . . . . 170

8.9 Systematic uncertainties in the $e \mu$ channel. . . . . . . . . . . . . 171

8.10 Summary of the measured uncertainties . . . . . . . . . . . . . 178

D.1 Table projecting the expected number of signal and background events in Run II. . . . . . . . . . . . . . . . . . . . . . . . . . . . . . . . . 199

D.2 Class I exporiment using the $Z \rightarrow$ ee sample. . . . . . . . . . . . . . . 201

D.3 Class I experiment using the $Z \rightarrow \mu \mu$ sample. . . . . . . . . . 202

D.4 Class II cxperiment using the $2000 Z \rightarrow$ ee events. . . . . . . . . . . 203

D.5 Class II cxperiment using the $1000 Z \rightarrow \mu \mu$ events. . . . . . . . . . . 203

D.6 Class I experiment using 288 non-hadronic $Z \rightarrow \tau \tau$ event sample. . . 204

D.7 Class II experiment using 288 non-hadronic $Z \rightarrow \tau \tau$ event sample. . . 204

D.8 Class I experiment using 2112 hadronic $Z \rightarrow \tau \tau$ event sample. . . . . 205

D.9 Class II cxperiment, using 2112 hadronic $Z \rightarrow \tau \tau$ cvent sample. . . . . 205

E.1 Assignment of calorimeter cell $\eta$ indices into TT $\eta$ indices. . . . . . . 206

F.2 Assignment of calorimeter cell $\phi$ indices into TT $\phi$ indices. . . . . . . 207

G.1 Statistic of template distributions used in the analysis . . . . . . . 221 
J.1 Results from simulated ensembles with 10\% background contamination. 211

J.2 Results from simulated ensembles with $\sim 19 \%$ background contamination. . . . . . . . . . . . . . . . . . 245

J.3 Results from simulated ensembles with $\sim 28 \%$ background contamination. . . . . . . . . . . . . . . . . 246

J.1 Results from simulated ensembles with $\sim 37 \%$ background contamination. . . . . . . . . . . . . . . . . 246

J.j Results from simulated cnsembles with (fixcd) $20 \%$ background con-

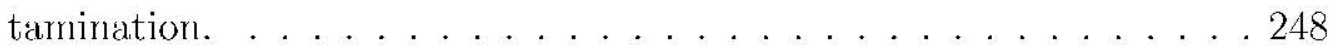

J.6 Results from simulated ensembles with (fixed) $40 \%$ background con-

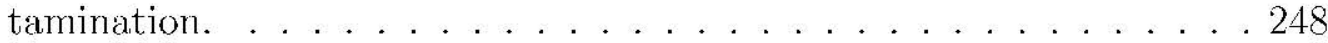

K.1 Four vectors of objects: event 1997007 in run 168393. . . . . . . . . . 250

K.2 Four vectors of objects: event 8710859 in run 174901. . . . . . . . . . 251

K.3 Four vectors of objects: cvent 15259654 in run $177826 . \quad$. . . . . . . . 251

K.4 Four vectors of objects: event 37315438 in run 178159 . . . . . . . . . 252

K.5 Four vectors of objects: event 8735139 in run 178733. . . . . . . . . 252

K.6 Four vectors of objects: cvent 11709332 in run $179141 . \quad$. . . . . . . . 252

K.7 Four vectors of objects: event 26386170 in run 179195 . . . . . . . . . 252

K.8 Four vectors of objects: event 19617819 in run 179331. . . . . . . . . 253

K.9 Four vectors of objects: event 121971122 in run $166779 \ldots$. . . . . . . 253

K.10 Four vectors of objects: cvent 13869716 in run 177681 . . . . . . . . 253

K.11 Four vectors of objects: event 26229014 in run 178152. . . . . . . . . 253

K.12 Four vectors of objects: event 13511001 in run 178177. . . . . . . . . 254

K.13 Four vectors of objects: cvent 14448436 in run $180326 . . . . .254$ 


\section{List of Figures}

2.1 Box diagram for the $B^{0} \rightarrow \bar{B}^{0}$ transition. . . . . . . . . . . . 11

2.2 leading order Feynman diagrams for the $e^{+} e^{-} \rightarrow b \bar{b}$ process. . . . . . 12

2.3 NLO Feynman diagrams for the $e^{+} e^{-} \rightarrow b \bar{b} . \ldots \ldots \ldots \ldots$

2.4 Fermion triangle diagram which could cause an anomaly. . . . . . 13

2.5 Feynman diagrams representing the processes which contribute to the mass of the $W$ boson. . . . . . . . . . . . . . . . . . 16

2.6 l seading order Feynman diagrams for the $t \bar{t}$ pair production. . . . . . 18

2.7 Leading order Feynman diagrams for the top quark production via weak interaction. . . . . . . . . . . . . . . 18

2.8 NLO $t \bar{t}$ production cross-section as a function of the mass of the top

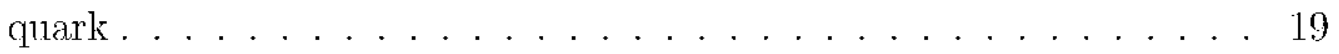

2.9 Probability of occurrences of the $t \bar{t}$ final-states. . . . . . . . . 21

3.1 Direct measurements of the mass of the $t$ quark. . . . . . . . . 23

3.2 Current world average for the mass of the t quark. . . . . . . 23

3.3 Results of the mass of the $W$ boson. . . . . . . . . . . . . 24

3.4 The $W$ boson as a function of the mass of the $t$ quark as well as the mass of the Higgs boson. . . . . . . . . . . . . . 26

3.5 Global $\chi^{2}$ fit to SM parameters. . . . . . . . . . . . . 31 
1.1 A schematic of the FNAL Tevatron complex. . . . . . . . . . . 35

4.2 A schematic of the $\mathrm{D} \varnothing$ detector in the $x-y$ plane $\ldots \ldots \ldots \ldots$

1.3 A schematic outlining the principles of operation of the unit SMT detector. . . . . . . . . . . . . . . . 44 43

4.4 The transverse view of the layout of the DØ tracking system. . . . . . 44

1.5 A transverse vicw of one quadrant of the Forward Preshower detector. 16

4.6 A quadrant of the $\mathrm{D} \varnothing$ calorimeter in the $x-y$ plane. . . . . . . 48

1.7 A read-out cell of the $\mathrm{D} \emptyset$ calorimeter. . . . . . . . . . . . 19

4.8 The layout of the Muon detector at 1$) \varnothing$ in the $x-y$ plane. . . . . . 51

5.1 Schematic illustration of the sequenees in a generic event generator. . 56

5.2 The proton parton distribution functions. . . . . . . . . 58

5.3 A schematic showing the $2 \rightarrow 2$ scattering process for a proton antiproton collision. . . . . . . . . . . . . . . . . 59

6.1 A sketch representing the signature of a typical $e \mu$ candidate event within the detector. . . . . . . . . . . . . 66

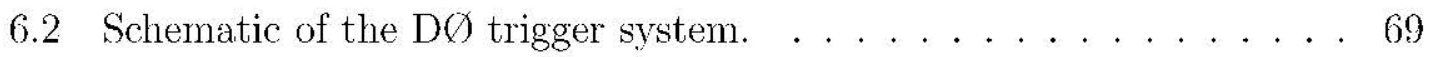

$7.1 \quad Z$ resonance from Tevatron data and simulated events. . . . . . . . . 92

7.2 The invariant mass reconstruction from di-muon cvents (central muon

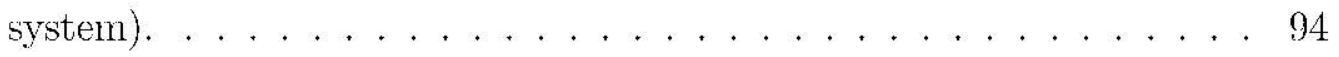

7.3 Invariant di-muon mass obtained from di-muon pairs detected in the forward muon system. . . . . . . . . . . . . . . . . 95

7.4 A schematic representing the stages in the evolution of a jet from

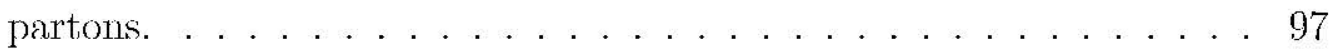

$7.5 E_{T}$ density per unit $\eta \times \phi$ as a function of detector $|\eta| \ldots \ldots . . .99$ 
7.6 Jet cnergy response for a 0.7 cone algorithms. . . . . . . . . . 100

7.7 Average energy density from $\gamma+$ jet events in central calorimeter. . . . 101

7.8 The energy spectrum of partons from the $b$ quark. as well as the light quarks. . . . . . . . . . . . . . . . . . . . . 104

7.9 Energy distribution of light-quark partons versus the reconstructed cnergy. . . . . . . . . . . . . . . . 105

7.10 The distribuion of the fractional electron energy resolution versus clectron encrgy in the central calorimeter. . . . . . . . . 110

7.11 The distribuion of the fractional electron energy resolution versus electron energy in the end-cap calorimeters. . . . . . . . . . . . . . 111

7.12 Muon $1 / p_{T}$ residuals for some $\Delta p_{T}$ and $\Delta \eta$ ranges. . . . . . . 113

$7.13 \sigma_{1 / p_{T}}$ of the muons as a function of $1 / p_{T}$ for the central region. . . . 114

$7.11 \sigma_{1 / p_{T}}$ of the muons as a function of $1 / p_{T}$ for the forward region. . . . 115

7.15 Parameteric representation of the resolution of jet energy scale. . . . 117

8.1 A schematic of the di-lepton event topology. . . . . . . . . . . 120

$8.2 \Lambda$ representation of the 3 -momentum for the $t \rightarrow b W, W \rightarrow l \nu_{l}$ decay scquence. . . . . . . . . . . . . . . . . . . . . 123

8.3 The geometrical representation of the paraboloid surface for all physical values of all $\vec{t}$ momenta. . . . . . . . . . . . . . . . . . . . . 124

8.4 Solutions for the $\vec{t}$ and $\vec{t}$ projected on to the laboratory transverse momentum planc. . . . . . . . . . . . . . . . . 126

8.5 Weight distribution of a simulated signal event. . . . . . . . . 131

8.6 An example of the maximum $\log$ likelihood fit. . . . . . . . . . 136

8.7 Statistical uncertainty from the $\log$ likclihood fit. . . . . . . . 138 
8.8 Template distributions for analysis in the $e \mu$ channel. . . . . . . . . 112

8.9 More template distributions for analysis in the $e \mu$ channel. . . . . . . 143

8.10 Some template distributions for analysis in the ee channcl. . . . . . . 111

8.11 Histogram of the $m_{p c a k}$ distribution from the selected data events in the $e \mu$ channel. . . . . . . . . . . . . . . . . . . . . . . . 145

8.12 Histogram of the $m_{\text {peak }}$ distribution from the sclected data events in the di-electron channel. . . . . . . . . . . . . . . . . . . . 146

8.13 Histogram of the most likely values from 100 simulated ensembles each with eight everts. . . . . . . . . . . . . . . . . . 148

8.14 Fast MC ensemble calibration using Pythia events . . . . . . . . . . . 152

8.15 Fast MC ensemble calibration using Pythia events . . . . . . . . . . 153

8.16 Ensemble calibration in the $e \mu$ channel. . . . . . . . . . . . . . . 155

8.17 Signal only ensemble calibration using di-clectron events. . . . . . . 156

8.18 Ensemble calibration in the di-electron channel. . . . . . . . . . . . 157

8.19 The maximum likelihood fit to the $e \mu$ data cnscmble. . . . . . . . . 160

8.20 The maximum likelihood fit to the di-electron data ensemble. . . . . . 160

8.21 The combined signal and background template for the $e \mu$ channel. . . 161

8.22 The combined signal and background template for the ee channel. . . 162

8.23 The log-likelihood distribution (after bias correction) as a function of the input MC value of the top quark mass, for the cnscmble of di-electron events. . . . . . . . . . . . . . . . . 164

8.24 The distribution of MLEs from unique and simulated e $\mu$ ensembles, the signal events having input $\mathrm{MC} m_{t}=175 \mathrm{GoV}$. . . . . . . . . 166

8.25 The distribution of MLEs from unique and simulated ee ensembles, the signal events having input $\mathrm{MC} m_{t}=175 \mathrm{GoV} . \ldots . . . . .167$ 
8.26 The combined log-likelihood distribution for the $e \mu$ and the di-clectron data ensembles. . . . . . . . . . . . . . . . . 168

8.27 Calibration curve from signal $e \mu$ ensembles with jet encrgy scaled higher. . . . . . . . . . . . . . . . . 173

8.28 Calibration curve from signal $e \mu$ ensembles with jet energy scaled lower. . . . . . . . . . . . . . . . . . . . 173

8.29 Calibration curve from $e \mu$ ensembles (signal \& background processes) with the jet encrgy scaled higher. . . . . . . . . . . . . . 171

8.30 Calibration curve from e $\mu$ ensembles (signal \& background processes) with the jet energy scaled lower. . . . . . . . . . . . . . . . . . 174

8.31 Calibration curve from signal di-electron ensembles with jet energy scaled higher. . . . . . . . . . . . . . . . . . 175

8.32 Calibration curve from signal di-clectron ensembles with jet encrgy scaled lower. . . . . . . . . . . . . . . . . . . . . . . . . 175

8.33 Combined results from the disscrtation. . . . . . . . . . . . . 182

9.1 Previous di-lepton channcl results from $\mathrm{CDF}^{\prime}$ and $\mathrm{D} \varnothing \ldots \ldots \ldots . . .184$

9.2 Current results from the CDF and $\mathrm{D} \emptyset$ collaboration. . . . . . . . 187

9.3 Current results from the CDF collaboration. . . . . . . . . . . 188

E.1 Sketch showing the supcrposition of the $E_{T}$ spectrum. . . . . . . . 208

F.2 Cartoon of a typical turn-on-curve. . . . . . . . . . . . . . 208

E.3 Frequency with which various CEM TTs have the highest $E_{T}$ for the CEM(1,15) trigger in runs 150408 and $150409 \ldots$. . . . . . . . 209

F.4 Frequency with which various CEM TTs have second highest $E_{T}$ for the CEM $(2,10)$ trigger in runs 150408 and $150409 \ldots \ldots$. . . . . 209 
E.5 Frequency distributions of maximum TT in a run. . . . . . . . . . . 210

E.6 Maximum $E_{T}$ spectrum of the TTs in the region of interest . . . . . . 211

E.7 The CEM1s turn-on curve . . . . . . . . . . . . . . . . . . . . 212

F.8 The turn-on curves for some TTs in an unbiased measurement of the CEM10 and CEM5 triggers using precision readout from the TTs. . . 213

E.9 The turn-on curves for some individual TTs. . . . . . . . . . . . . . 211

E.10 The turn-on curves for CEM15, CEM10 and CEM5 triggers after the removal of defective TTs. . . . . . . . . . . . . . . . . . 215

F.11 The corrected turn-on curve for CFM10 and CEM5 triggers overlaid with results from the trigger-simulator. . . . . . . . . . . . . 216

F.1 Reconstructed $W$ boson mass from simulated cvents. . . . . . . . . . 219

F.2 Reconstructed $t$ quark mass from simulated events. . . . . . . . . . . 220

I.1 Results from $e \mu$ ensemble tests: input $m_{t}=140 \mathrm{GeV} . \ldots . . . .233$

I.2 Results from $e \mu$ ensemble tests: input $m_{t}=160 \mathrm{GeV} \ldots \ldots . . .234$

I.3 Results from $e \mu$ cnscmble tests: input $m_{t}=175 \mathrm{GcV}$. . . . . . . . 235

I.4 Results from $e \mu$ ensemble tests: input $m_{t}=190 \mathrm{GeV}$. . . . . . . . . 236

I.j Rosults from $e \mu$ cnscmble tosts: input $m_{t}=210 \mathrm{GoV} \ldots \ldots . . . . .237$

I.6 Results from ee ensemble tests: input $m_{t}=140 \mathrm{GeV}$. . . . . . . . . 238

I.7 Results from ee cnscmble tests: input $m_{l}=160 \mathrm{GcV}$. . . . . . . . . 239

I.8 Results from ee ensemble tests: input $m_{t}=175 \mathrm{GeV} . . .2 . .240$

I.9 Results from ee ensemble tests: input $m_{t}=190 \mathrm{GeV}$. . . . . . . . . . 241

I.10 Results from ee ensemble tests: input $m_{l}=210 \mathrm{GeV} . \ldots . . .212$

J.1 Calibration curve for simulated cnscmbles with $10 \%$ background contamination. . . . . . . . . . . . . . . . . . 244 
J.2 Calibration curve for simulated ensembles with $\sim 19 \%$ background

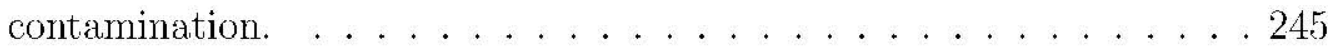

J.3 Calibration curve for simulated ensembles with $\sim 28 \%$ background contamination. . . . . . . . . . . . . . . . 246

J.4 Calibration curve for simulated ensembles with $\sim 37 \%$ background contamination. . . . . . . . . . . . . . . 247

J.5 Calibration curve for simulated ensembles with fixed $20 \%$ background contamination. . . . . . . . . . . . . . . . . . 249

J.6 Calibration curve for simulated ensembles with fixed $40 \%$ background contamination. . . . . . . . . . . . . . . . . . . . 249

L.1 Weight distributions of di-clectron channel candidate events. . . . . 255

L.2 Weight distributions of the remaining candidat events in the di-electron channel. . . . . . . . . . . . . . . . . . . . . . 256

L.3 Weight distributions of candidate events selected in the $e \mu$ channcl. . 257

L.4 Weight distributions of remaining candidate events selected in the $e \mu$ channcl. . . . . . . . . . . . . . . . . . 258 


\section{List of Abbreviations}

\begin{tabular}{|c|c|}
\hline Abbreviation & The complete phrase \\
\hline $\mathrm{CC}$ & Central Cryostat (Central Calorimeter) \\
\hline C.L. & Confidence Limit \\
\hline $\mathrm{CDF}$ & Collider Detector at Fermilab \\
\hline CEM TT(s) & Central Electromagnetic Trigger Tower(s) \\
\hline CERN & $\begin{array}{l}\text { Conseil Europeane de la Recherche Nucléaire, aka } \\
\text { Organisation Européenne pour la Recherche Nucléaire } \\
\text { (European Organization for Nuclear Research) }\end{array}$ \\
\hline $\mathrm{CH}^{\prime \prime} \mathrm{I}$ & Contral Fiber Tracker \\
\hline CPS & Central Preshower \\
\hline DCA & Distance of Closest Approach \\
\hline $\mathrm{EC}$ & End-cap Calorimeter \\
\hline ECAL & Elcctromagnetic(EM) Calorimetcr \\
\hline FNAT, & Fermi National Accelerator Laboratory \\
\hline FPS & Forward Preshower \\
\hline $\mathrm{GcV}$ & giga clcctron volt i.e. $10^{6} \mathrm{cV}$ \\
\hline
\end{tabular}




\section{(continuation)}

\begin{tabular}{|c|c|}
\hline Abbreviation & The complete phrase \\
\hline HCAL & Hadronic Calorimeter \\
\hline ICD & Inter-Cryostat Detector \\
\hline LEPEWWG & LEP ElcctroWcak Working Group \\
\hline $\mathrm{I}, \mathrm{HC}$ & Large Hadron Collider \\
\hline L.O. & $\begin{array}{l}\text { Leading Order (pertaining to physical } \\
\text { interactions expressed via Feynman diagrams) }\end{array}$ \\
\hline $\mathrm{MC}$ & Monte Carlo \\
\hline MLE & Maximum Likelihood Estimate \\
\hline MTC & Muon Track in Calorimeter \\
\hline$m_{t}$ & the mass of the top quark \\
\hline N.L.O. & Next-to-Leading Order \\
\hline N.N.I.O. & Next-to-Next-to-I eading Order \\
\hline $\mathrm{PCA}$ & Point of Closest Approach \\
\hline Preacc & Pre-Accelerator \\
\hline $\mathrm{QCD}$ & Quantum Chromodynamics \\
\hline RMS & (square-)Root of the Mean of Squares \\
\hline
\end{tabular}




\section{(continuation)}

\section{Abbreviation The complete phrase}

$\begin{array}{ll}\text { SM } & \text { (The) Standard Model (of Particle Physics) } \\ \text { SMT } & \text { Silicon Microstrip Tracker } \\ \text { stat. } & \text { statistical uncertainty } \\ \text { syst. } & \text { systcmatic uncertainty } \\ \text { TeV } & \text { tera electron volt i.e. } 10^{9} \mathrm{eV} \\ \text { TI(s) } & \text { Trigger Lower(s) } \\ \text { VI.PC } & \text { Visible light photon counter }\end{array}$




\section{Chapter 1}

\section{Introduction}

Most objects have atoms as their structural and functional unit. This was cstablished by Dalton[1] back in the early nineteenth century. By the 1920s experiments performed independently by Rutherford[2], Bohr[3], Geiger along with Marsden[1], Chadwick[5] and others[6] helped establish that atoms have sub-structure. Atomic electrons orbit the nucleus, whose constituents are protons and neutrons. The above mentioned experiments were performed by directing a beam of energetic charged particles (e.g. alpha particles, and beta particles) called the projectile, onto a target. The interaction of the projectile particles with the target caused the former to scatter in different directions. $\Lambda$ particle detector was placed around the target ${ }^{1}$ to measure the projectile's scattering angle. In these experiments an energetic stream of alpha particles (from a radioactive material) was used to 'probe' the atoms ${ }^{2}$. Since that era, the particle physicist's quest has been to learn about the fundamental building blocks of matter and their interactions.

The science of elementary particle physics helped us formulate a complete un-

\footnotetext{
${ }^{1}$ These target atoms (e.g. Au) were much more massive compared to the projectile

${ }^{2}$ The wave particle duality[7] was known by then.
} 
derstanding of the atom and its constituents. This scicnce took a giant leap lorward with the invention of the particle accelerator[8]. A contemporary particle accelerator is a machine which generates and accelerates particles to relativistic specds. Although we can now reach much higher energies than before, the strategy to probe by bombarding elementary particles remains the same. For the experiment relevant to this dissertation, the distance scale probed is nearly $10^{-16}$ metcrs. Collisions at such extreme energies are sufficient to cause interactions among the constituents of the proton and the anti-proton.

\subsection{The frontier of particle physics}

The Standard Model (S.M) [9] ol particle physics has stood the test of decades of stringent experimental scrutiny. However, it has not been proved that the S.M is a complete and sel[-consistent description of clementary particles[10]. According to the SM the top quark is one of the fundamental building blocks of matter. The value of the mass of the top quark is one issue that the SM does not address. This value is a frec parameter of the model. It is established cxperimentally.

Fermilab is the only place in the world where one can study top quarks until the Large Hadron Collider comes up at CERN. At Formilab, the collisions between a proton and an anti-proton are used to generate a pair of top and anti-top quarks. The first phase of the experimental program (Run I) began nearly fifteen years ago and the program culminated in the discovery of the top quark[11], as well as a precise measurement of its mass at nearly $180 \mathrm{GeV}$ with an uncertainty of about $5 \mathrm{GeV}[12]$. A sccond phase (Run II) in the cxpcrimental program began nearly four ycars ago, in spring 2001. The prime objective of the current program is the answer to the 
question: does the SM Higgs boson exist? We hope to answer this question here at the Tevatron. A measurement of the mass of the top quark to a greater precision than what was achicved in Run $I$ is another important objective for Run II. In the context of the SM, a more precise measurement of the mass of the top quark will allow us to indirectly constrain the mass of the Iliggs boson better than before ${ }^{3}$. This thesis is among the first fow to present measurcments of the mass of the top quark at the Tevatron in Run II.

Currently, at the Tevatron the mass of the top quark is measured from the decay of the top and anti-top quark pairs. The decay of these pairs can be via three principal modes. One of these modes is the di-lepton channel. In this channel, the final-state of the top and anti-top decay has two charged leptons ${ }^{4}$. This dissertation describes a measurement of the mass of the top quark using events consistent with the S.M hypothesis that the top and anti-top quark decay via the di-lepton channcl.

\subsection{An outline of the dissertation}

The layout of this thesis is outlined below. The next chapter, Chapter 2 introduces the theoretical framework needed to interpret the results to follow. The consequences of the measurement of the mass of the top quark in the context of the SM are illustrated in Chapter 3. Chapter 4 is devoted to the description of the Tevatron collider and the D $\varnothing$ detector at Fermilab. In Chapter 5 the tools based on computing resources and their applications in generating simulated events are outlined. The systematic and careful procedure of selecting candidate events from a large data

\footnotetext{
${ }^{3}$ This will be discussed later in Chapter 2.

${ }^{4} \mathrm{An}$ electron or a muon is only considered. The tau lepton decays before it interacts with the detector.
} 
set is described in Chapter 6. Chapter 7 illustrates the basic principles used in calibrating the kinematic quantities which are of interest in this analysis. The method of extracting the mass of the top quark is described in Chapter 8. The analysis algorithm is applied to numerous simulated events for self consistency tests. Then the mass of the top quark is measured using the selected candidate events. The statistical and systematic uncertaintics associated with the measurement are discussed. A comparison with other measurements is discussed in Chapter 9, along with the the implications of a precision measurement for Run II. Conclusions and the outlook for the future are presented in Chapter 10.

For the completion of this dissertation my personal contribution were manifold. They range from hardware efforts, software development and data analysis. With regards to this dissertation I was involved in establishing the out-of-cone showering corrections for jets. For the first time in Run II, the average corrections to jet 1vectors were established to represent the parton 4-vectors. A dynamical likelihood fitting algorithm was designed and implemented for analysis of data as well as simulated events. All these tasks were accomplished for this dissertation. A summary of some personal efforts during my Ph.D. program are highlighted in Appendix B. This has been a wonderful and an enjoyable collaborative venture.

\subsection{Conventions and terminology}

In this dissertation, unless otherwise stated, the units used to represent the energy of the fundamental particles is in $\mathrm{GeV}$. Following a common convention, the speed of light in vacuum $(c)$ is set to a dimensionless value of unity. Therefore, the units used to represent the momentum and mass of fundamental particles are GeV. 
In this disscrtation, the uncertainty in the statistic generally denotes the uncertainty in the mean measurement within $\sim 68 \%$ confidence limit. 


\section{Chapter 2}

\section{Theoretical Context}

'This chapter addresses some theoretical issues relevant to the phenomenology of the top quark. Some of its properties, which include the production and decay modes, are discussed in the context of the Standard Model (SM).

\subsection{Synopsis of the Standard Model of particle physics}

The mathematical framework which describes the dynamics of the elementary particles is the SM[9]. The constituents of this model are assumed to be point-like particles.

According to the SM, the fiundamental constituents of matter are fermions. There are 3 generations (families) of quarks and leptons (these are fermions). The top quark $(t)$ and the bottom quark $(b)$ constitute one such generation. The fermions interact with one another via the exchange of gauge bosons. The gauge bosons are the mediators of the fundamental interactions. The gluon $(g)$ is the mediator of the 


\begin{tabular}{|c|c|c|c|c|}
\hline $\begin{array}{c}\text { Particle } \\
\text { class }\end{array}$ & $\begin{array}{l}\text { name } \\
\text { (symbol) }\end{array}$ & $\begin{array}{l}\text { mass } \\
(\mathrm{GeV})\end{array}$ & $\begin{array}{c}\text { woak } \\
\text { iso-spin }\end{array}$ & $\begin{array}{c}\text { cm } \\
\text { charge } \\
\text { (e) }\end{array}$ \\
\hline \multirow{5}{*}{$\begin{array}{l}\text { Gauge } \\
\text { Bosons }\end{array}$} & photon $(\gamma)$ & 0 & - & 0 \\
\hline & $W^{+}$ & 80.2 & - & +1 \\
\hline & $Z$ & 91.2 & - & 0 \\
\hline & gluon $(g)$ & 0 & - & 0 \\
\hline & Higgs & $?$ & - & 0 \\
\hline \multirow{6}{*}{$\begin{array}{c}\text { Quarks } \\
\text { (fractional } \\
\text { charge } \\
\text { fermion) }\end{array}$} & down $(d)$ & $\sim 1 \times 10^{-2}$ & $-1 / 2$ & $-1 / 3$ \\
\hline & strange $(s)$ & $\sim 2 \times 10^{-1}$ & $-1 / 2$ & $-1 / 3$ \\
\hline & bottom $(b)$ & $\sim 4.5 \times 10^{0}$ & $-1 / 2$ & $-1 / 3$ \\
\hline & $u p(u)$ & $\sim 5 \times 10^{-3}$ & $+1 / 2$ & $+2 / 3$ \\
\hline & charm (c) & $\sim 1.5 \times 10^{0}$ & $+1 / 2$ & $+2 / 3$ \\
\hline & top $(t)$ & $\sim 1.8 \times 10^{2}$ & $+1 / 2$ & $+2 / 3$ \\
\hline Leptons & elcctron $(e)$ & $\sim \check{\jmath} .11 \times 10^{-4}$ & $-1 / 2$ & -1 \\
\hline (integer & muon $(\mu)$ & $\sim 1.06 \times 10^{-1}$ & $-1 / 2$ & -1 \\
\hline $\begin{array}{l}\text { charge } \\
\text { fermion) }\end{array}$ & $\tan (\tau)$ & $\sim 1.78 \times 10^{0}$ & $-1 / 2$ & -1 \\
\hline Leptons & electron neutrino $\left(\nu_{e}\right)$ & $<3 \times 10^{-9}$ & $+1 / 2$ & 0 \\
\hline (neutral & muon neutrino $\left(\nu_{\mu}\right)$ & $<1.9 \times 10^{-4}$ & $+1 / 2$ & 0 \\
\hline fermion) & tau neutrino $\left(\nu_{\tau}\right)$ & $<1.8 \times 10^{-2}$ & $+1 / 2$ & 0 \\
\hline
\end{tabular}

Table 2.1: Some properties of the constituent particles of the SM of particle plyysics. Each of the quarks come in 3 color familics. The set of S.M particles is listed here.

strong interaction, the $W^{ \pm}$and $Z$ bosons mediate the weak interaction, and the photon $(\gamma)$ mediates the electromagnetic interaction. The SM incorporates the physics of three of the four fundamental forces, viz. the strong force, the weak force and the electromagnetic force. Appropriate internal symmetries associated with physical observations have been identificd and they form the core of the mathematical formulation of the S.I. A unitary group $U(1)$, having quantum number $Y$, represents the weak hyper-charge symmetry. The special unitary group $S U(2)$, describes the the 'left-handed' $(L)$ weak iso-spin interactions. Lastly, the $S U(3)$ group describes the symmetries of the strong interaction, the quantum numbers of which are denoted 
by $C$. Collectively this results in a $S U_{C}(3) \times S U_{L}(2) \times U_{Y}(1)$ symmetry[9], [13],[14]. However, we know from nature that the $S U_{L}(2) \times U_{Y}(1)$ symmetry is not exact, but is broken spontancously to yicld electromagnetic interactions represented by $U_{E M}(1)$. This is manifest in the varied mass spectrum of the particles.

The SM succeeds in unifying the electromagnetic and weak interactions into a single clectro-weak interaction. These interactions come about if one demands that the Lagrangian be invariant under $S U_{L}(2) \times U_{Y}(1)$ symmetry. A problem that appears is that the mass terms for the gange bosons and fermions break the symmetry if added arbitrarily. The Higgs mechanism solves this problem. When an additional potential energy density term is added to the original Lagrangian density then the mass terms for the weak gauge bosons and fermions can be accommodated without breaking the symmetry[14]. The potential energy density term is

$$
V(\phi)=m^{2}\left(\phi^{\dagger} \phi\right)+\lambda\left(\phi^{\dagger} \phi\right)^{2}
$$

where $\phi$ is the complex scalar Higgs field. This gives rise to an additional massive (scalar) particle, the Higgs boson, which interacts with the gauge bosons and fermions involved in electro-weak interaction. All fermion masses in the S.h, including that of the $t$ quark, come as free parameters. We can establish these parameters cxperimentally. For a more exhaustive discussion on the S.M numcrous references are indicated here[15]. In the next sub-section the SM frec parametcrs are discussed. 


\subsubsection{The free parameters in the Standard Model}

Some of the free parameters of the SM are the:

- gauge couplings associated with the three independent gauge groups which manilest the weak, the electromagnetic and the strong intcractions,

- parameters which describe the Higgs potential,

- Yukawa type couplings between the IIiggs boson and SM fermions,

- CKM mixing parancters which relate the weak cigenstates to the strong eigenstates.

All SM free parameters are not experimental observables. Pseudo-parameters are used to re-express the free parameters in terms of experimental observables. The set of pscudo-parameters relevant to the measurements in the electro-weak sector are the:

- E.M coupling constant $\left(\alpha_{E M}\right)$,

- strong coupling constant $\left(\alpha_{s}\right)$,

- gauge boson masses $\left(M_{W}, M_{Z},\right)$,

- Higgs boson mass $\left(m_{h},\right)$,

- fermion masses.

It is known that, except for the mass of the top quark, all other fermion mass terms are very small compared to the energy collisions of interest in this thesis. Therefore, in interactions involving high momentum transfer (the 4-vector of which is denoted by $Q$ ) there are cssentially six parameters of interest. They are the: 
- EM coupling constant, $\alpha_{E M}\left(Q^{2} \approx M_{Z}^{2}\right)$,

- strong coupling constant, $\alpha_{s}\left(Q^{2} \approx M_{Z}^{2}\right)$,

- boson mass terms, $M_{W}, M_{Z}$, and $m_{h}$,

- top quark mass $m_{t}$.

For describing the physics of collisions involving high momentum transfers, the parameter $\alpha_{E M}$ is most dependent on the mass of the top quark $\left(m_{t}\right)$. from among all quarks. Therefore, $\alpha_{F M}$ is calculated as a function of $m_{t}$ and then added explicitly

to the five flavor $\alpha_{E M}$, which is denoted by $\alpha_{E M}^{(5)}\left(Q^{2} \approx M_{Z}^{2}\right)$. This is then taken as an input parameter for the SM. Similarly, for calculations involving $\alpha_{\text {had }}$, contributions from the five flavors are accounted for by $\alpha_{\text {had }}^{(5)}\left(Q^{2} \approx M_{Z}^{2}\right)$.

We now diseuss some issues pertaining to the SM which hinted at the existence of the $t$ quark before its discovery.

\subsubsection{Evidence for the existence of the top quark}

The discovery of the top quark in 1995 at the Tevatron[11] was not accidental. Before its discovery, experimental results hinting at its existence were available. The hints were consistent with the theory of the SM as well. T'his sub-section motivates some of this indirect evidence. The experiments were done at energies below the threshold for the $t$ quark production. The experimental evidence was based on the absence of flavor-changing neutral currents in $B$ meson decays and the measurement of the weak isospin of the $b$ quark. Furthermore, the absence of triangle anomalies provide theoretical consistency. 
A. Measurement of $\mathbf{B}^{0}-\overline{\mathbf{B}}^{0}$ mixing
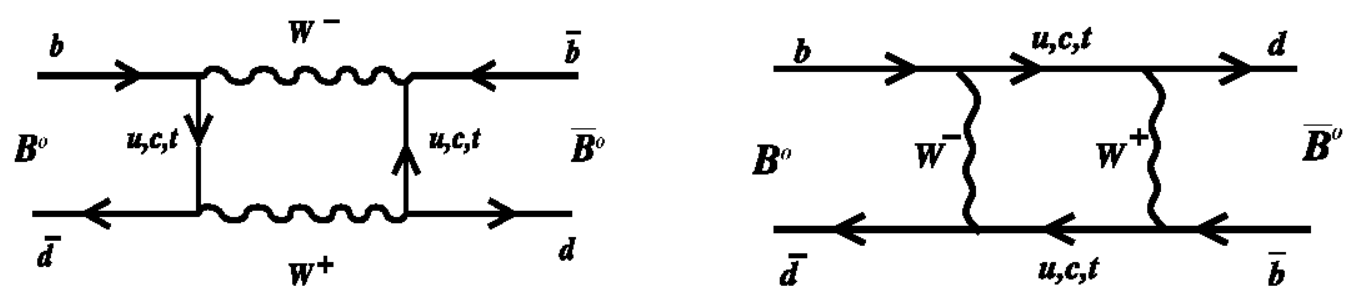

Figure 2.1: Box diagram for the $B^{0} \rightarrow \bar{B}^{0}$ transition. These Feynman diagrams illustrate the mixing in the $B$ meson sector, and the loop contribution from the $t$ quark is dominant since it is most massive compared to the others.

The $B^{0}$ and $\bar{B}^{0}$ mesons can $\operatorname{mix}[16]$ with each other through the interactions represented by the box diagrams in Figure 2.1. In order to match experimental data involving the level of $B^{0}-\bar{B}^{0}$ mixing it was necessary that the $l$ quark exist, and that its mass $\left(m_{t}\right)$ was constrained to be $m_{t}>45 \mathrm{GeV}[17]$. It was, however, possible to have models in which quarks from lower mass states contribute to the observed high levels of $B^{0}-\bar{B}^{0}$ mixing[18]. Hence, this evidence was not sufficient.

\section{B. Measurement of the forward-backward asymmetry $A_{F B}$ at $Z$ resonance and the partial decay width $\Gamma(\mathrm{Z} \rightarrow \mathrm{b} \overline{\mathbf{b}})$}

The forward-backward asymmetry in $e^{+} e^{-} \rightarrow b \bar{b}$ at the $Z$ boson resonance helped in investigating the iso-spin doublet nature of the $b$ quark. Figure 2.2 is the leading order contribution to $e^{+} e^{-} \rightarrow b \bar{b}$. However corrections from processes as shown in Figure 2.3 contribute as well. In the electroweak sector of the SM, particles are grouped into $S U_{L}(2)$ weak isospin multiplets. 'The helicity states associated with a left-handed particle $p$ have weak isospin quantum number $T_{3 L}^{p}$, and it can be measured under certain conditions. The process $e^{+} e^{-} \rightarrow b b$ can proced via $e^{+} e^{-} \rightarrow \gamma^{*} \rightarrow b \bar{b}$ as well as $e^{+} e^{-} \rightarrow Z \rightarrow b \bar{b}$. The interference between these two processes results in an asymmetric angular distribution for $b$ production. The value 
of the weak isospin quantum number $T_{3 L}^{b}$ for the $b$ quark influcnecs the amount of asymmetry. In particular the coupling of the $b$ quark to the $Z$-boson is proportional to $\left(T_{3 L}^{b}+\frac{1}{3} \sin ^{2} \theta_{W}\right)$, where $\theta_{W}$ is the woak mixing angle. For a wcak isospin singlet state $T_{3 L}^{b}=0$, the coupling would be +0.07 . However, for a doublet component of the weak isospin $\left(T_{3 L}^{b}=-0.5\right)$, one obtains a value of -0.43 . The experimentally detcrmined value of $T_{3 L}^{b}$, from $e^{+} e^{-} \rightarrow b \bar{b}$ below the $Z$ pole, is $-0.504{ }_{-0.011}^{+0.018}[19]$. This substantiated the claim that the $b$ quark is part of a weak isospin doublet, with the $l$ quark as its partner.

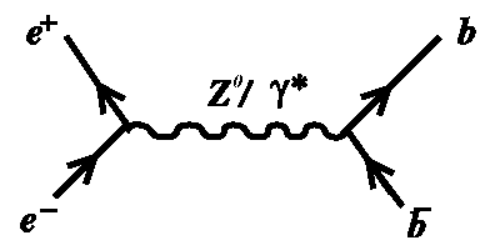

Figure 2.2: Leading order Feynman diagrams for the $e^{+} e^{-} \rightarrow b \bar{b}$ process.
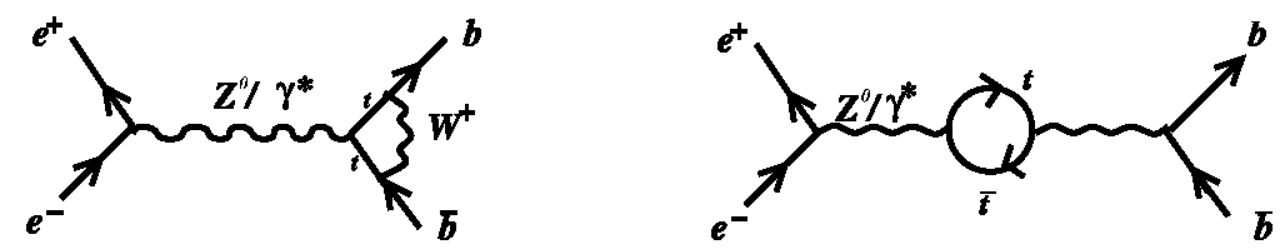

Figure 2.3: Next-to-leading-order Feynman diagrams for the $e^{+} e^{-} \rightarrow b \bar{b}$.

Precision measurements of the width $\Gamma_{Z}$ of the $Z$ boson have been made at LFP. Consider the production of $b \bar{b}$ via the decay of the $Z$ boson represented in Figure 2.2. The measurement is done at the $Z$ resonance production threshold, $e^{+} e^{-} \rightarrow Z \rightarrow b \bar{b}$.

The effect on the partial width $\Gamma_{Z \rightarrow b \bar{b}}$ due to the top quark, is due to the next to leading order process illustrated in Figure 2.3. 


\section{Absence of flavor-changing neutral current decays}

One of the most important features of the SM is the Glashow Iliopoulos Maiani (GIM) [22] mechanism which leads to the absence of flavor changing neutral current ( $\mathrm{FCNC}$ ) transitions at the tree level and the suppression of FCNC transitions at the one loop level in the quark sector. A large set of experimental limits on rare processes can be cxplained via this mechanism. This mechanism requires the presence of a second generation of quark pairs, the charm and the strange quarks.

Before the discovery of the second or third generation quarks, it was experimentally observed that the decay $K_{L}^{0} \rightarrow \mu^{+} \mu^{-}$was very rare:

$$
\frac{\Gamma\left(K_{L}^{0} \rightarrow \mu^{+} \mu^{-}\right)}{\Gamma\left(K_{L}^{0} \rightarrow \text { all modes }\right)} \approx 9 \times 10^{-9}
$$

However, with the introduction of a sccond generation of quarks it was possible to theoretically explain this feature.

The treatment could be extended to incorporate a third gencration of quark pairs. The existence of three pairs of quarks along with three pairs of leptons was significant in theory, since it could help explain the absence of certain 'triangle anomalies:

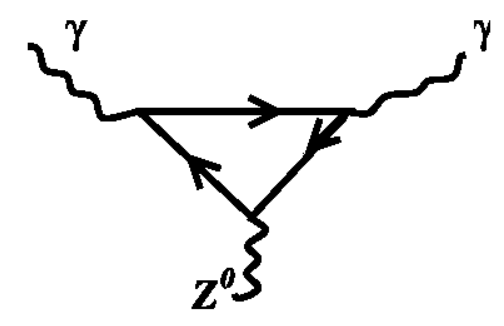

Figure 2.4: $\Lambda$ fermion (quark or charged lepton) triangle diagram which could causc an anomaly. 


\section{Absence of triangle anomalies}

In the electro-weak sector of the $\mathrm{SM}$, contribution from a triangular loop leads to a divergence. Consider the triangle diagram illustrated in Figure 2.1. The anomaly is proportional to the strength of the coupling of the weak neutral current times the square of the charge of the fermion. For a theory which is re-normalizable, the contributions from these diagrams must be zero. It can be shown that if the number of quark generations and the number of lepton generations is equal, then the anomaly will cancel out. This argument is the simplest way by which we can avoid the anomaly, but it is not necessarily the only one. Hence this lone argument for the existence of the $t$ quark is insufficient.

\subsection{Some fundamental properties of the top quark}

The top quark was discovered barcly 10 ycars ago[11]. 'The SM top quark

- is a fermion, with spin $1 / 2$,

- has electromagentic charge $+\frac{2}{3}$ times the electromagnetic charge of the electron, - has 1 unit of color charge.

These above mentioned characteristics were assigned even before the discovery of the top quark. However, these properties have not been verified for the signal events we call the top quark. Along with the above characteristics, it is also known that:

- the current world average value of its mass is measured to be $178.0 \pm 4.3 \mathrm{GeV}[20]$, - its mass is known to a much better relative precision than the masses of the light quarks,

- from the knowledge of its mass, it can be prodictcd that it decays in about $10^{-25} \mathrm{~s}$, before it can hadronize. This makes it possible to study the properties of the direct 
decay products of the $l$ quark without much influence of the strong interaction.

\subsection{Significance of the top quark mass}

Yukawa type couplings relate the matter content of the SM to the Higgs field. The top quark mass $\left(m_{t}\right)$ is related to the Higgs vacuum expectation value $v$ by $m_{l}=Y \frac{v}{\sqrt{2}}$, where $Y$ is the Yukawa coupling. Since $v \approx 216 \mathrm{GcV}$ and $m_{l} \approx 178 \mathrm{GcV}$ it yields the coupling constant $Y \approx 1$. A unity value of the coupling constant may perhaps yicld insight to physics that is not supported by the SM.

\subsubsection{An indirect consequence of the top mass: radiative corrections and indirect constraints}

In the SM, higher order (radiative) corrections to clectro-weak processes and self-energy terms depend on the mass of the $t$ quark, as well as mass of the Higgs boson via the Feynman loop diagrams. Consider the EW parancter $\rho$, which can be expressed as $[21]$

$$
\rho=\frac{M_{W}^{2}}{M_{Z}^{2}\left(1-\sin ^{2} \theta_{W}\right)} \equiv 1+\Delta r
$$

The contribution duc to radiative effects can be re-cxpressed as ${ }^{1}$ :

$$
\Delta r=\Delta r_{1}+\Delta r_{2}+\ldots
$$

Each of the above terms represent contributions involving higher order loop corrections from other EW parameters. In this context, it has been established[15]

\footnotetext{
${ }^{1}$ In the simplistic Born approximation the radiative eflects are absent and $\Delta r=0$.
} 

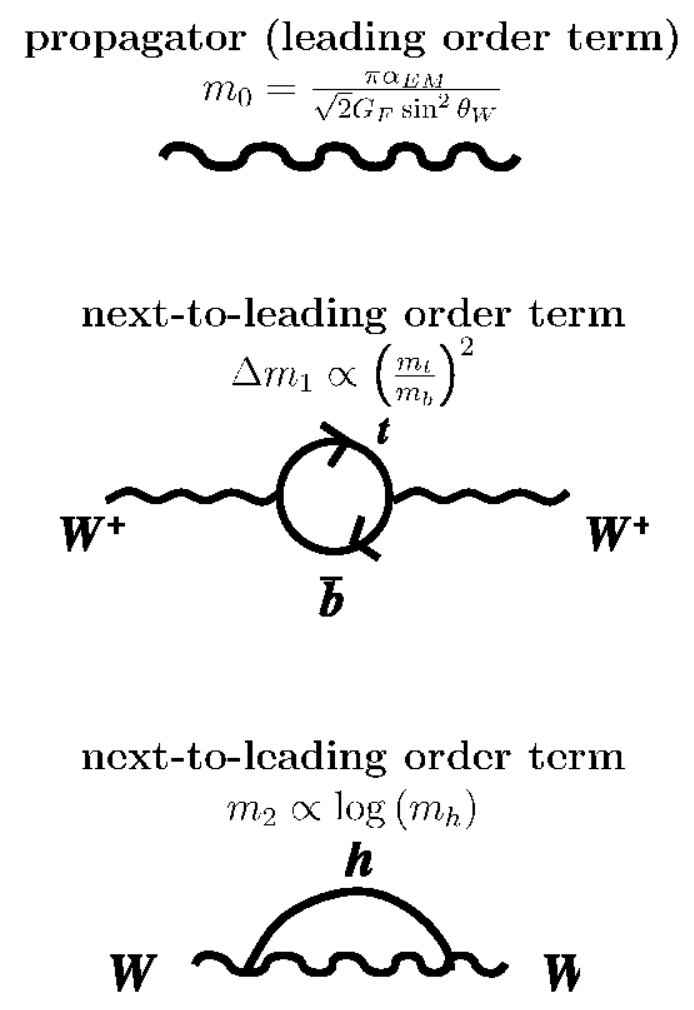

Figure 2.5: Feynman diagrams representing the processes which contribute to the mass of the $W$ boson. 'The mass (sell-energy) of the $W$ boson $M_{W}$ is represented as $M_{W}=m_{0}+\Delta m\left(m_{t}^{2} / m_{b}^{2}\right)+\Delta m\left(\log \left(m_{h}\right)\right)+$ higher order corrcctions.

that

$$
\Delta r_{1}=\frac{3 G_{F}}{8 \sqrt{2} \pi^{2}} m_{t}^{2}
$$

and,

$$
\Delta r_{2}=\frac{\sqrt{2} G_{H}}{16 \pi^{2}} M_{W}^{2}\left[\frac{11}{3} \ln \left(\frac{m_{h}^{2}}{M_{W}^{2}}\right)+\ldots\right]
$$

These radiative corrections are very sensitive to the mass of the top quark and are less sensitive to the mass of the ITiggs boson. If they were sufficiently sensitive, then by now we would know more about the mass of the Higgs boson.

As an cxample let us consider the precision mass measurement of the $W$ boson. 
The mass (sell-energy) of the $W$ boson can be attributed to the propagator term $\left(m_{0}\right)$, as well as loop contributions. Figure 2.5 describes the leading order (propagator tcrm) and the next-to-leading order (one loop diagrams) contributions that involve the mass term of the $W$ boson. The mass of the $W$ boson is expressed as a sum of contributions from these Feynman diagrams as:

$$
M_{W}=m_{0}+\Delta m_{1}+\Delta m_{2}+\ldots=m_{0}+\Delta m\left(\frac{m_{l}^{2}}{m_{b}^{2}}\right)+\Delta m\left(\log \left(m_{h}\right)\right)+\ldots
$$

Therefore, the electroweak corrections to the $W$ boson mass have a quadratic dependenec of the $l$ quark mass and a logarithmic dependence on the mass of the Higgs boson.

The ratio of the mass of the $t$ quark to that of the $b$ quark enters as the quadratic correction. The $t$ quark is nearly 40 times more massive than the $b$ quark. Therefore, the contribution from the $\Delta m_{1}$ term, which is proportional to $\left(\frac{m_{l}^{2}}{m_{b}^{2}}\right)$, is the dominant correction term compared to the logarithmic contribution, $\Delta m_{2}$, which is due to the mass of the Higgs boson ${ }^{2}$. If a precision measurement of the $W$ boson mass as well as the $t$ quark mass is obtained, we can constrain the Higgs mass better than what is known currently[12].

\subsection{Top quark production in proton anti-proton collisions, and their subsequent decay modes}

At the Tevatron the top quark is produced via the strong interaction as well as the weak interaction. However, the production of $t \bar{t}$ quark pairs occurs via the strong

\footnotetext{
${ }^{2}$ Quadratic terms $\sim G_{h}^{2} m_{h}^{2}$ only appear for two loop diagrams involving virtual Higgs boson, and their effects are too small.
} 

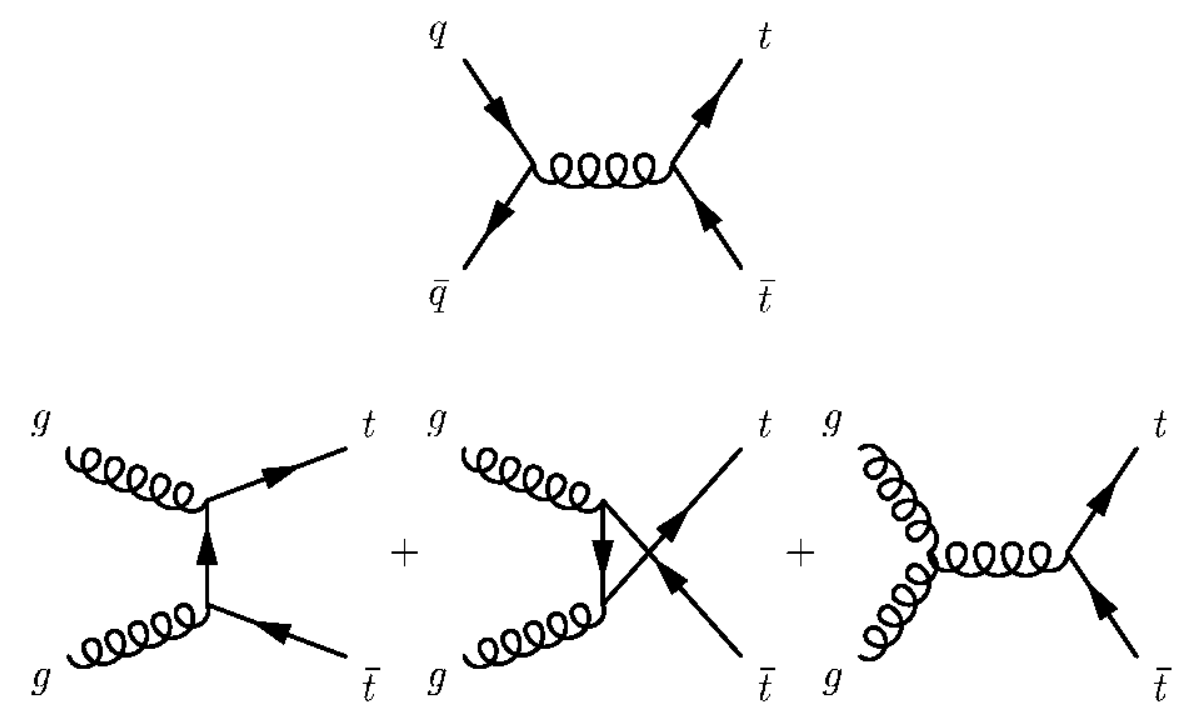

Figure 2.6: Leading order Feynman diagrams for the $t \bar{t}$ pair production. At the center-of-mass of $1.96 \mathrm{TeV}$, nearly $85 \%$ of the time the production mechanism is via $q \bar{q}$ annihilation (the diagram on the top), while the gluon-gluon fusion represents the remaining $15 \%$. The proton and anti-proton (valence) quarks are represented symbolically by $q$ and $\bar{q}$ respectively.
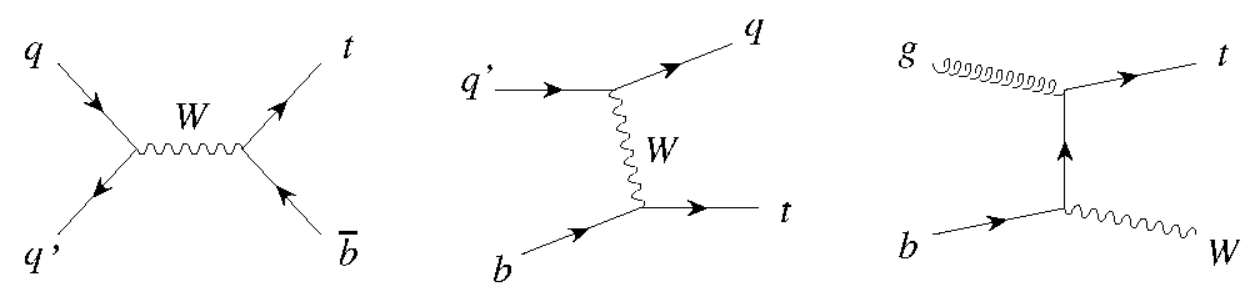

Figure 2.7: Leading order Feynman diagrams for the top quark production via weak interaction.

interaction. The leading order Feynman diagrams for the pair-production are shown in Figure 2.6. Production of a single $t$ quark occurs via the weak interaction. The weak processes arc illustratcd in Feymman diagrams in Figure 2.7. For this analysis, we rely on the $t \bar{t}$ pair production process. At $1.96 \mathrm{TeV}$ center-of-mass energy, nearly $85 \%$ of the $t \bar{t}$ pairs are produced by quark anti-quark annihilation, and the rest are produced via gluon-gluon fusion. 


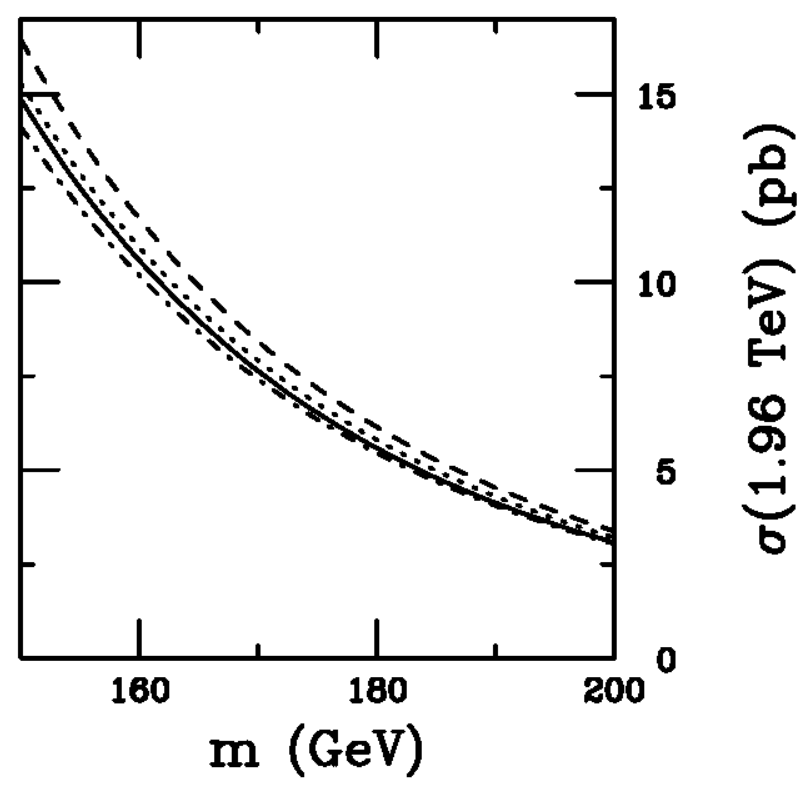

Figure 2.8: Next-to-next-to leading order $t \bar{t}$ production cross-section as a function of the $t$ quark mass. This plot is obtained from [23].

The SM $t(\bar{t})$ quark primarily decays into the $W^{+}\left(W^{-}\right)$boson and a $b(\bar{b})$ quark ${ }^{3}$. Threfore, the characterization of the decay channels of the $t \bar{t}$ quark is done following the subsequent decay channcls of the $W^{ \pm}$boson. Table 2.2 illustrates the branching fraction of the $W^{ \pm}$pair into hadrons and leptons. Two-thirds of the time the $W$ boson decays hadronically, while the remaining onc-third of the time it decays into charged leptons and their corresponding neutrinos ${ }^{4}$. When both the $W$ bosons (from the $t \bar{t}$ pair) decay into either $e$ and/or $\mu$ then the decay channel is called the di-lepton channel. This channel constitutes nearly $4.8 \%$ of the $t t$ decay. The chances of occurrences of all $t \bar{t}$ decay modes are graphically represented in Figure. 2.9. When both the $W$ bosons decay to electrons, then the final-state is the di-electron channel, but when they decay into mons then the final-state is the

\footnotetext{
${ }^{3}$ Nearly $99.9 \%$ of the time. In the SM, $t \rightarrow c W$ decay occurs nearly $0.001 \%$ of the time.

${ }^{4}$ From now onward, unless otherwise stated, reference to particles will also imply reference to their anti-particles.
} 


\begin{tabular}{|c|c|c|c|c|}
\hline & $\begin{array}{c}W^{+} \rightarrow e^{+} \nu_{e} \\
(1 / 9)\end{array}$ & $\begin{array}{c}W^{+} \rightarrow \mu^{+} \nu_{\mu} \\
(1 / 9)\end{array}$ & $\begin{array}{c}W^{+} \rightarrow \tau^{+} \nu_{\tau} \\
(1 / 9)\end{array}$ & $\begin{array}{c}W^{+} \rightarrow q q^{\prime} \\
(6 / 9)\end{array}$ \\
\hline $\begin{array}{c}W^{-} \rightarrow e^{-} \nu_{c} \\
(1 / 9)\end{array}$ & $1 / 81$ & $1 / 81$ & $1 / 81$ & $6 / 81$ \\
\hline $\begin{array}{c}W^{-} \rightarrow \mu^{-} \nu_{\mu} \\
(1 / 9)\end{array}$ & $1 / 81$ & $1 / 81$ & $1 / 81$ & $6 / 81$ \\
\hline $\begin{array}{c}W^{-} \rightarrow \tau^{-} \nu_{\tau} \\
(1 / 9)\end{array}$ & $1 / 81$ & $1 / 81$ & $1 / 81$ & $6 / 81$ \\
\hline $\begin{array}{c}W^{-} \rightarrow q^{\prime} \bar{q} \\
(6 / 9)\end{array}$ & $6 / 81$ & $6 / 81$ & $6 / 81$ & $36 / 81$ \\
\hline \hline
\end{tabular}

Table 2.2: Possible decay modes for the $W^{+} W^{-}$daughter pair from the $t t$ pair.

di-muon channel. However, when they decay to an electron and a muon, then the decay constitutes the $e \mu$ channel. 


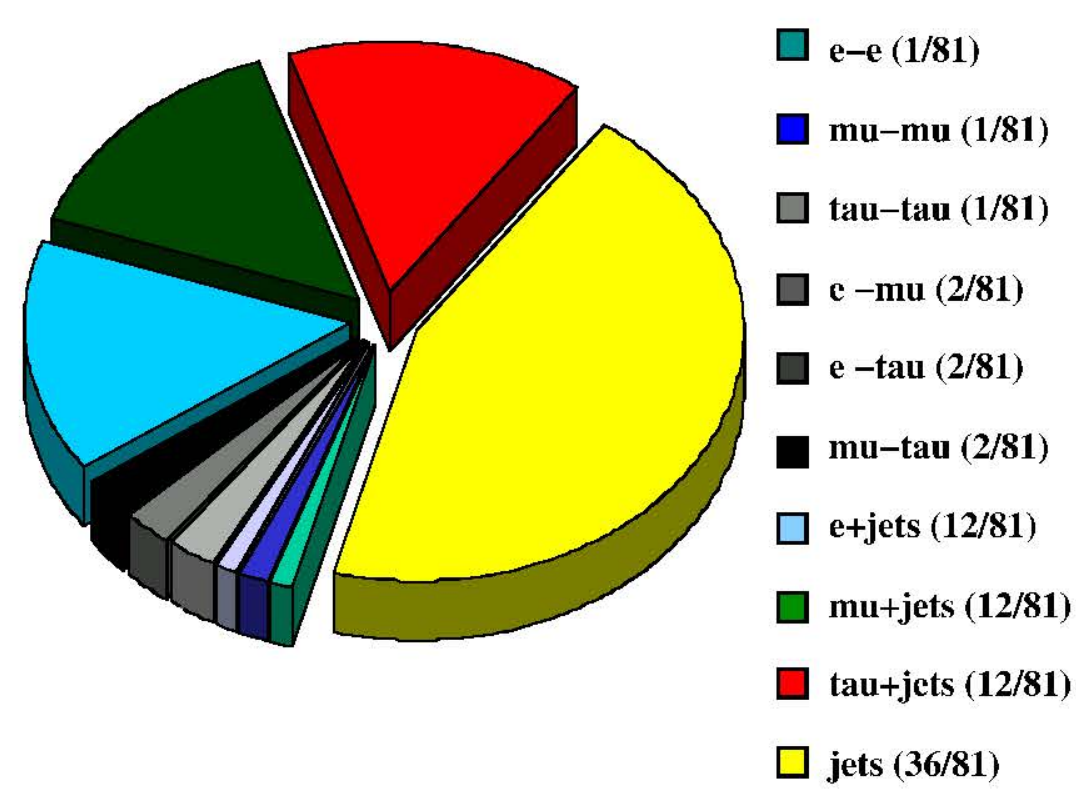

Figure 2.9: Probability of oceurrences of the $l \bar{l}$ final-states. The dominant decay mode $(\sim 11.4 \%)$ is to the all jets channel, while the (charged) lepton + jets channcl has noarly $28 \%$ contribution. The least likely decay mode is the (charged) di-lepton channel, which get only about $4.8 \%$ of the total occurrences. 


\section{Chapter 3}

\section{Experimental Context}

'This chapter develops an experimental perspective from the underlying theoretical concepts of the electro-weak (EW) parameters of the Standard Model (SM) already discussed. Some of these parameters are deterministic and are used to constrain other undetermined parameters.

\subsection{SM measurements in the EW sector}

The measurements of the mass of the $t$ quark and the $W$ boson are illustrated in this scetion.

\subsubsection{The mass of the top quark}

Figure 3.1 shows various direct measurements of the mass of the top quark at the Tevatron by the CDF and DØ cxperiments in Run I. The Run I measurement of the $t$ quark mass in the di-lepton channel by the 100 experiment was $168.4 \pm 12.3$ (stat.) \pm 3.6 (syst.) GeV[27]. The single most precise moasurement of the mass of the $t$ quark is $180.1 \pm 5.3 \mathrm{GeV}[12]$. 


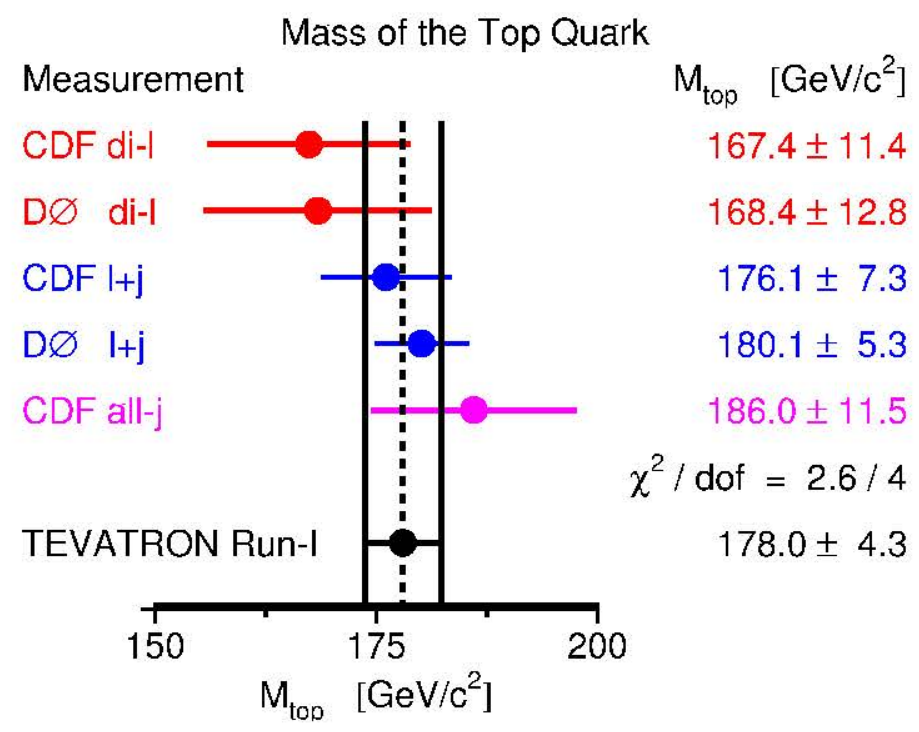

Figure 3.1: Direct measurements of the mass of the $t$ quark. Results from the measurement of the mass of the top quark are illustrated from direct measurements by the I) $\varnothing$ and the CISF experiments in various channels.

\section{Top-Quark Mass [GeV]}

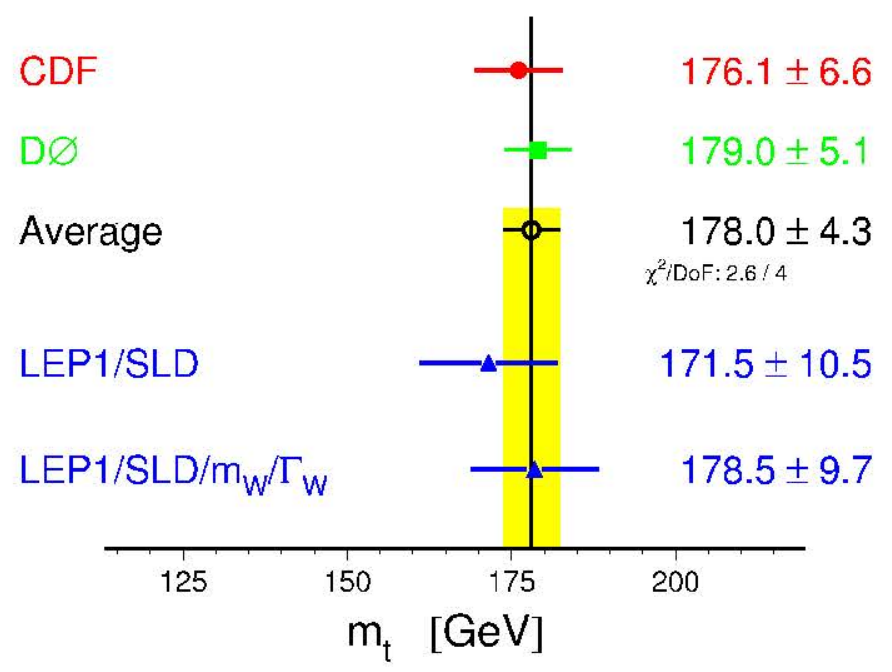

Figure 3.2: Current world average for the mass of the $t$ quark. This is the winter 2004 result from the Tevatron EW working group[25] and the LEP EW working group [21] 


\subsubsection{The mass of the $W$ boson}

\section{W-Boson Mass [GeV]}

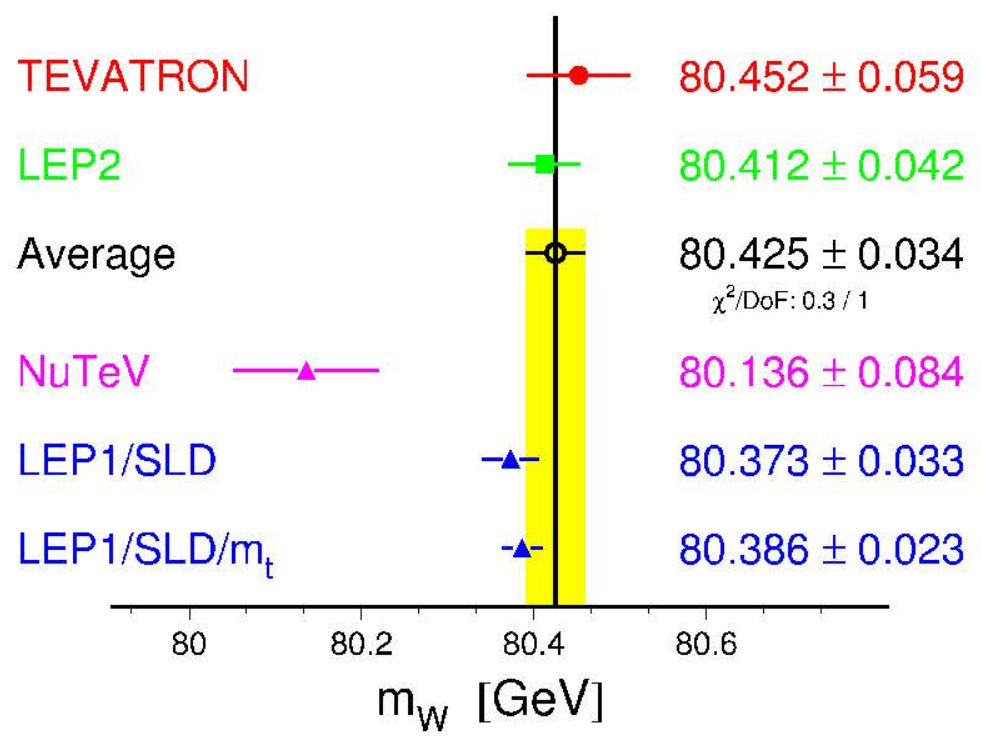

Figure 3.3: Results of the mass of the $W$ boson from LEPEWWG Results of the mass of the $W$ boson from the LEP electroweak working group[24].

Figure 3.3 illustrates the currently known information of the mass of the $W$ boson from independent experiments. The current world average from the direct as well as indirect measurcments is $80.412 \pm 0.042 \mathrm{GcV}[24]$.

Although direct measurements are possible for measuring the mass of the $t$ quark, it is of interest to check the self consistency of the SM by establishing indirect constraints from independent experiments. Figure 3.2 illustrates the measurements of the mass of the t quark which are used to extract the current, world average. These come from indirect constraints from the SM as well as from direct measurements just discussed. The current world average for the mass of the $t$ quark from the LEP clcctroweak working group[24] and the Tevatron clectroweak working group[25] is $178.0 \pm 4.3 \mathrm{GeV}[20]$. 
A precision measurement of the $W$ boson mass $\left(M_{W}\right)$ along with the top quark mass $\left(m_{t}\right)$ can be used to constrain the mass of the Higgs boson $\left(m_{h}\right)$. Figure 3.4 shows the plot of the mass of the $W$ boson versus the mass of the $l$ quark. Hypothetical values of the mass of the Higgs boson are illustrated as the shaded bands overlaid in the $m_{t}-M_{W}$ space. From current indirect measurements the $68 \%$ confidence level (CL) contour for a consistent set of $M_{W}$ and $m_{t}$ is shown as the dark line. The dotted contour indicates the set obtained via direct measurements at a $68 \%$ C.L. (for cither one of the parameters). Such constraints can be made tighter with more precise measurements of the $W$ boson as well as the top quark. The region overlapping the two contours is the region consistent with both direct as well as indirect constraints for a set of values of $m_{t}, M_{W}$ and $m_{h}$. 


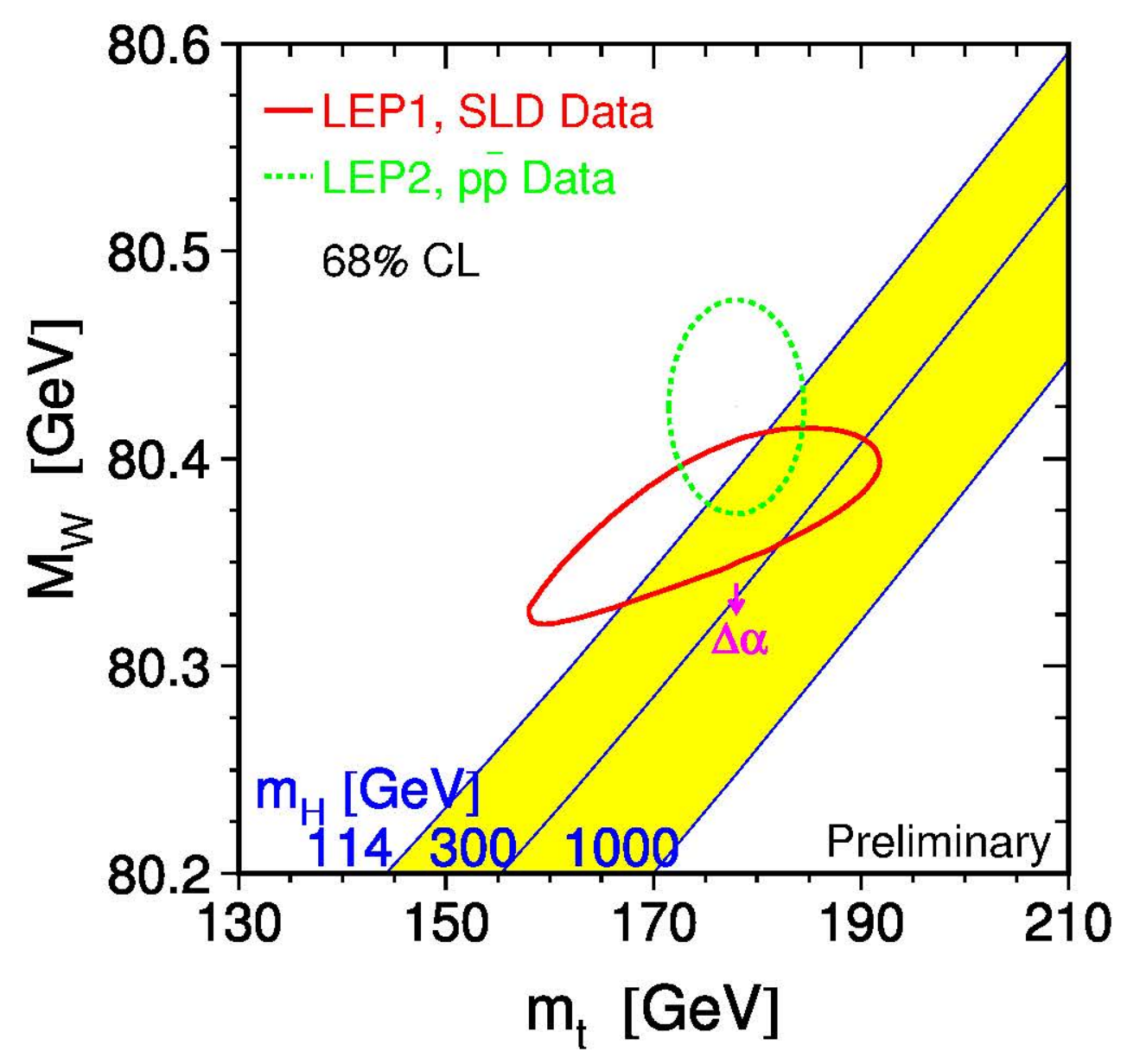

Figure 3.4: The mass of the $W$ boson expressed as a function of the mass of the $t$ quark and the mass of the Higgs boson. The mass of the $t$ quark is parametrically represented along the horizontal axis, and it ranges from $130 \mathrm{GeV}$ to 210 $\mathrm{GeV}$. The mass of the Higgs boson is parameterized along the shaded (yellow) band ranging from $114 \mathrm{GeV}$ to $1000 \mathrm{GeV}$. The combined LEP2 and the Tevatron data is represented by the dotted (green) contour, while the LEP1 and the SLD results are represented by the continuous (red) contour. While the former represents direct measurement of the mass of the $t$ quark, the latter represents an indirect measurement. This is the LEP Electroweak Working Group's (August) summer 2004 result. 


\subsection{SM analysis of the free parameters}

For the analysis of electro-weak data in the realm of the SM one uses a set of input parameters ${ }^{1}$. Some free parameters of the SM are less precisely known than others. The parameters $\alpha_{F M}\left(Q^{2} \approx M_{Z}^{2}\right), G_{F}$ and $M_{Z}$ are more precisely measured than $\alpha_{s}\left(M_{Z}\right), m_{u}, m_{d}$, and so on. One can trade a parameter which is less precisely known for another one which is better measured and this frectom is used to cxtract a set of the best measured ones as input parameters.

The contributions from the above mentioned parameters are replaced by QED running coupling at the $Z$ mass scale, $\alpha_{E M}\left(M_{Z}^{2}\right)$. The hadronic contribution to the running hadronic coupling constant at similar energy scales denoted by $\Delta \alpha_{\operatorname{had}}^{(5)}\left(M_{Z}^{2}\right)$, as illustrated in Table 3.1, is obtained through dispersion relations from data on $e^{+} e^{-} \rightarrow$ hadrons at low center-of-mass energies[33]. Using the input parameters of the SM, the radiative corrections can be established to a sufficient precision to match experimental accuracy. Theoretical predictions and measurements from data help derive constraints on some parameters, namely, $m_{t}, \alpha_{s}\left(M_{Z}^{2}\right)$, and $m_{h}$.

\footnotetext{
${ }^{1}$ As mentioned before in Chapter 2 , the masses and the couplings involved in the theory are ad-hoc
} 


\begin{tabular}{|l|l||c||c|}
\hline & Observable & . Measurement $(\mathrm{GeV})$ & S.M fit value $(\mathrm{GeV})$ \\
\hline \hline & $M_{Z}[\mathrm{GcV}]$ & $91.1875 \pm 0.0021$ & 91.1873 \\
& $\Gamma_{Z}[\mathrm{GcV}]$ & $2.1952 \pm 0.0023$ & 2.1965 \\
\hline & $\sin ^{2} \theta_{\mathrm{eff}}^{\text {lept }}\left(Q_{\mathrm{la} \mathrm{B}}^{\mathrm{had}}\right)$ & $0.2324 \pm 0.0012$ & 0.23140 \\
\hline \hline & $M_{W}[\mathrm{GcV}]$ & $80.425 \pm 0.034$ & 80.398 \\
& $\Gamma_{W}[\mathrm{GeV}]$ & $2.133 \pm 0.069$ & 2.094 \\
\hline & $m_{t}[\mathrm{GeV}](\mathrm{p} \overline{\mathrm{p}}[25])$ & $178.0 \pm 4.3$ & 178.1 \\
\hline & $\Delta \alpha_{\text {had }}^{(5)}\left(m_{\mathrm{Z}}^{2}\right)[33]$ & $0.02761 \pm 0.00036$ & 0.02768 \\
\hline
\end{tabular}

Table 3.1: Results of some electroweak precision measurements at high $Q^{2}$ from [26]. The first block shows the Z-pole measurements. The second block shows additional results from other experiments: the mass and the width of the $\mathrm{W}$ boson measured at the Tevatron and at LEP-2, the mass of the top quark measured at the Tevatron, and the the contribution to $\alpha\left(m_{\mathrm{Z}}^{2}\right)$ of the hadronic vacuum polarization. For the corrclations between the measurements, taken into account in the analysis[26]. The SM fit results are derived from the SM analysis of altogether 18 results, also including constants such as the Fermi constant $G_{\mathrm{F}}$ (fit 3 of Table 3.2), using the programs TOPAZ0 [31] and ZFITTER [32]. 


\begin{tabular}{|l||c|c|c|}
\hline Fit & 1 & 2 & 3 \\
\hline \hline Measurements & $M_{W}, \Gamma_{W}$ & $m_{t}$ & $m_{t}, M_{W}, \Gamma_{W}$ \\
$(\mathrm{GcV})$ & & & \\
\hline \hline$m_{t}(\mathrm{GeV})$ & $178.5_{-8.5}^{+11.0}$ & $177.2 \pm 4.1$ & $178.1 \pm 3.9$ \\
$m_{h}(\mathrm{GeV})$ & $117_{-62}^{+162}$ & $129_{-50}^{+76}$ & $113_{-42}^{+62}$ \\
$\log \left[m_{h}\right](\mathrm{GeV})$ & $2.07_{-0.33}^{+0.38}$ & $2.11 \pm 0.21$ & $2.05 \pm 0.20$ \\
$\alpha_{s}\left(M_{Z}\right)$ & $0.1187 \pm 0.0027$ & $0.1190 \pm 0.0027$ & $0.1186 \pm 0.0027$ \\
\hline$\chi^{2} / d o f$ & $16.3 / 12$ & $15.0 / 11$ & $16.3 / 13$ \\
\hline$M_{W}(\mathrm{MeV})$ & - & $80386 \pm 23$ & - \\
\hline
\end{tabular}

Table 3.2: Global Standard Model fits of electroweak parameters obtained from data. All fits use the $Z$ pole results and $\Delta \alpha_{\text {had }}^{(5)}\left(m_{Z}^{2}\right)$ as listed in Table 3.1, also including constants such as the Formi constant $G_{F}$. In addition, the measurements listed in cach column are included as woll. For fit 2, the cxpected $W$ mass is also shown. For details on the fit procedure, using the programs T'OPAZ0 [31] and ZFITTEIR[32]. More details can be found at [26] and [30]. This example is from Altarelli and Grunewald[29].

\subsubsection{The SM predictions}

The SM is tested by fitting the set of measured observables in order to extract the input parameters of the model. The probability of the fit is based on the $\chi^{2}$ value in the minimum and the number of degrees of frecdom. This is a yardstick to confirm the compatibility of the SM with all experimental results for the same set of input parameters. Having determined the input parameters, it is possible to calculate values for any obscrvable, measured or unmeasured.

Consider the example from Altarelli and Grunewald[29] shown in Table 3.2. In column 1 a fit of all $Z$ pole data in addition to the $M_{W}$ and $\mathrm{I}_{W}$ is presented. In column 2, the fit from all $Z$ pole data as well as the $m_{t}$ is presented, while in column 3 only $m_{h}$ is omitted from all other input parameters. The value of $m_{t}$ 
can be obtained indircetly from radiative corrections from column 1. From the fit we see that the extracted value of $m_{t}$ is in perfect agreement with the direct measurement in Table 3.1. Information from column 2 can be used to estimate $M_{W}$. The experimental measurement of $M_{W}$ in Table 1 is larger by about one standard deviation with respect to the value from the fit in column 2. From the fit in column 3 wc obtain $\log _{10}\left(m_{h}\right)=2.05 \pm 0.20$ which yiclds $m_{h}=113_{-42}^{+62} \mathrm{GcV}$.

Of particular interest is the constraint on the mass of the Higgs boson, because this is the only lundamental particle of the Standard Model which has not been observed yet. The Figure 3.5 shows the $\Delta \chi^{2}$ curve derived from high- $Q^{2}$ precision electroweak measurements, performed at LEP and by SLD, CDF, and D $\varnothing$, as a function of the Higgs boson mass, assuming the Standard Model to be the successful theory of the nature of elementary particles. The preferred value for its mass, corresponding to the minimum of the curve, is at $113 \mathrm{GcV}$, with an experimental uncertainty of $+62 \mathrm{GeV}$ and $-42 \mathrm{GeV}$ (at $68 \%$ confidence level derived from $\Delta \chi^{2}=1$ for the black line, thus not taking the theoretical uncertainty shown as the blue band into account). While this is not proof that the Standard-Model Higgs boson actually exists, it does provide a range of mass values for a possible discovery. The precision clectrowcak measurcments tell us that the mass of the Standard-Model Higgs boson is lower than about $237 \mathrm{GeV}$ (onc-sided 95 percent confidence level upper limit derived from $\Delta \chi^{2}=2.7$ for the shaded (bluc) band, thus including both the experimental and the theoretical uncertainty.

This thesis is a small step toward obtaining a more precise measurement of the mass of the top quark at the 'levatron in the near future. Indirectly, the more precise measurement will help constrain the mass of the Iliggs boson further, and help narrow its scarch in future particle physics experiments. 


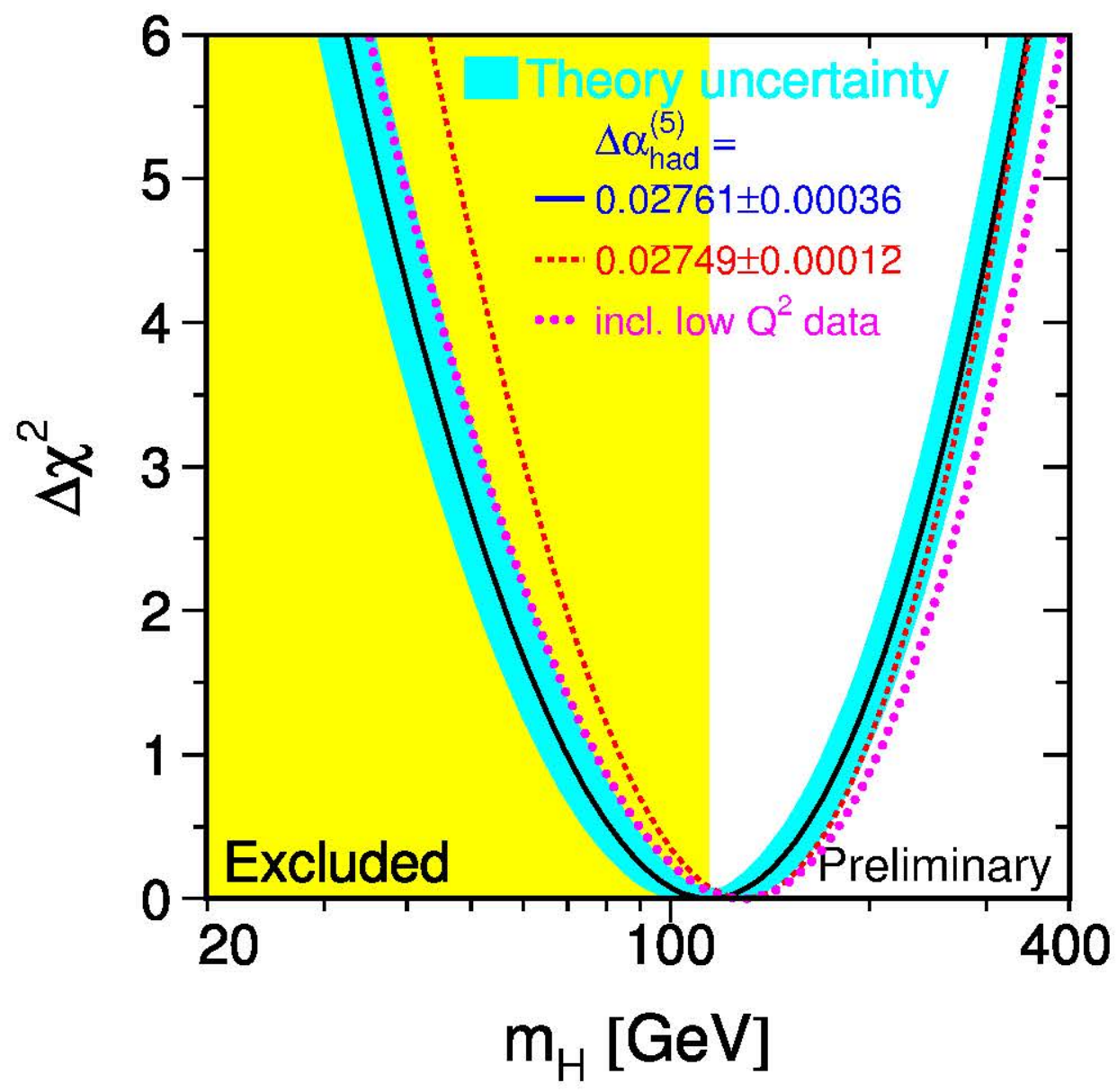

Figure 3.5: Global $\chi^{2}$ fit to all SM parameters except the mass of the Higgs boson, $m_{H}$. This is the summer 2004 result from the LFP Electroweak Working Group. The shaded (yellow) band is the range of hypothetical values of the mass of the Higgs boson which is excluded from our current experimental as well as theoretical knowledge of the SM. The fits are obtained using three input values of $\Delta \alpha_{\text {had }}^{(5)}$. The typical uncertainty in the fits is only shown for the continuous solid contour. The dis-continuos contours have uncertaintics which are similar in order of magnitude. 


\section{Chapter 4}

\section{The Experimental Setup}

The physics of clementary particles is studicd at specialized facilitics where clementary particle collisions are generated in controlled experiments. The work described in this thesis has becn done at one such facility, the Fermi National Accelcrator Laboratory (Fermilab).

\subsection{The Fermilab Tevatron accelerator}

The Tevatron at Fermilab[34], in Batavia, Illinois, is currently the world's most cnergetic particle accelcrator. In the carly 1990s the laboratory's main focus was the discovery of the top $(t)$ quark. The $t$ quark was discovered in 1995[11], and experiments continued collecting more data until 1997. The period of data-taking from the carly 1990 s to 1997 is called Run I. After an upgrade in the increased luminosity cnabled by the Main Injector, and the increased center-of-mass cncrgy $(\sqrt{s})$ of proton anti-proton collisions from $\sqrt{s}=1.8 \mathrm{TcV}$ to $\sqrt{s}=1.96 \mathrm{TcV}$, along with increased proton anti-proton bearn luminosity, Run II commenced in 2001. At the Tevatron Collider the focus of research on studies of interactions of protons and 
anti-protons continues at the highest encrgy fronticr.

\subsubsection{Generation and acceleration of protons and anti-protons}

Beams of protons and anti-protons are independently boosted to $980 \mathrm{GeV}$ energy in various stages. Some components involved in generating the highly relativistic beams are listed below and their role is discussed briefly.

- The Pro-accelcrator,

- the Lincar accelcrator,

- the Booster,

- the Main injector,

- the Anti-proton source,

- the Recycler, and

- the Tevatron.

The Pre-accelerator (Preace) is the source of $\mathrm{H}^{-}$ions which are eventually used to produce protons. The Preacc consists of a source of IIydrogen gas housed in an electrically charged dome. The source converts Hydrogen gas into $H^{-}$and this ionized gas is boosted to $750 \mathrm{keV}$ in a Cockroft-Walton accelerator. A continuous beam of $H^{-}$ions at $750 \mathrm{keV}$ is thus produced.

Lsing the beam of $\mathrm{H}^{-}$ions the Linear accelerator (Linac) boosts their energy by nearly 500 times to $400 \mathrm{MeV}$. The accelerator consists of copper cavities composed of drift tubes. The drift tubes are operated using power amplifiers generating radio frequency $(R F)$ signal voltage. RF voltage applied to the drift tube modules produce 
an clectric ficld which accelcrates the beam. Accoleration of the beam works much the same way as a parallel plate capacitor accelerates charged particles moving across it. As the velocity of the particles increases, the drift tubes (as well as the length of the gap between them) get larger. This allows acceleration of the beam of $\mathrm{H}^{-}$ions, in bunches. After the $I^{-}$beam is energized to $400 \mathrm{MeV}$ it is sent to either of the two sites:

(i) the Booster, for further acceleration, or

(ii) the Linac dump, lor beam tunc-up or diagnostic studies.

In the Booster the $400 \mathrm{MeV} H^{-}$ions are stripped of electrons, leaving only the proton core. The protons are then injected into the Booster synchrotron ring. The Booster is the first synchrotron, in the subsequent chain of accelerators. It consists of a series of magnets around a ring with a radius of nearly $75 \mathrm{~m}$ with 18 interspersed RF cavities. There are dipole magnets which are used to bend the trajectory of accelerating protons, while quadrupole magnets focus the particles into bunches. The electric ficld in RF cavities accelerate the beam to the high energy of $8 \mathrm{GeV}$, twenty times its initial energy. The beam is then led to the Main Injector (MI). The MI is a synchrotron nearly $530 \mathrm{~m}$ in radius with $18 \mathrm{RF}$ cavities. It boosts protons from energies of $8 \mathrm{GeV}$ to $150 \mathrm{GeV}$. However when the protons are used for producing anti-protons, the beam is then energized to $120 \mathrm{GeV}$ and led to the antiproton source from which $8 \mathrm{GcV}$ anti-proton bunches arc cxtracted (this is described in the next paragraph). These are led back into the MI where they are boosted to $150 \mathrm{GeV}$ just like the protons. Finally, the $150 \mathrm{GeV}$ proton and anti-proton beams are led from the MI to the Tevatron.

The beam of $120 \mathrm{GeV}$ proton bunches from the $\mathrm{MI}$ is led to the Target station for producing anti-protons. The proton bunches are smashed into a fixed nickel target 


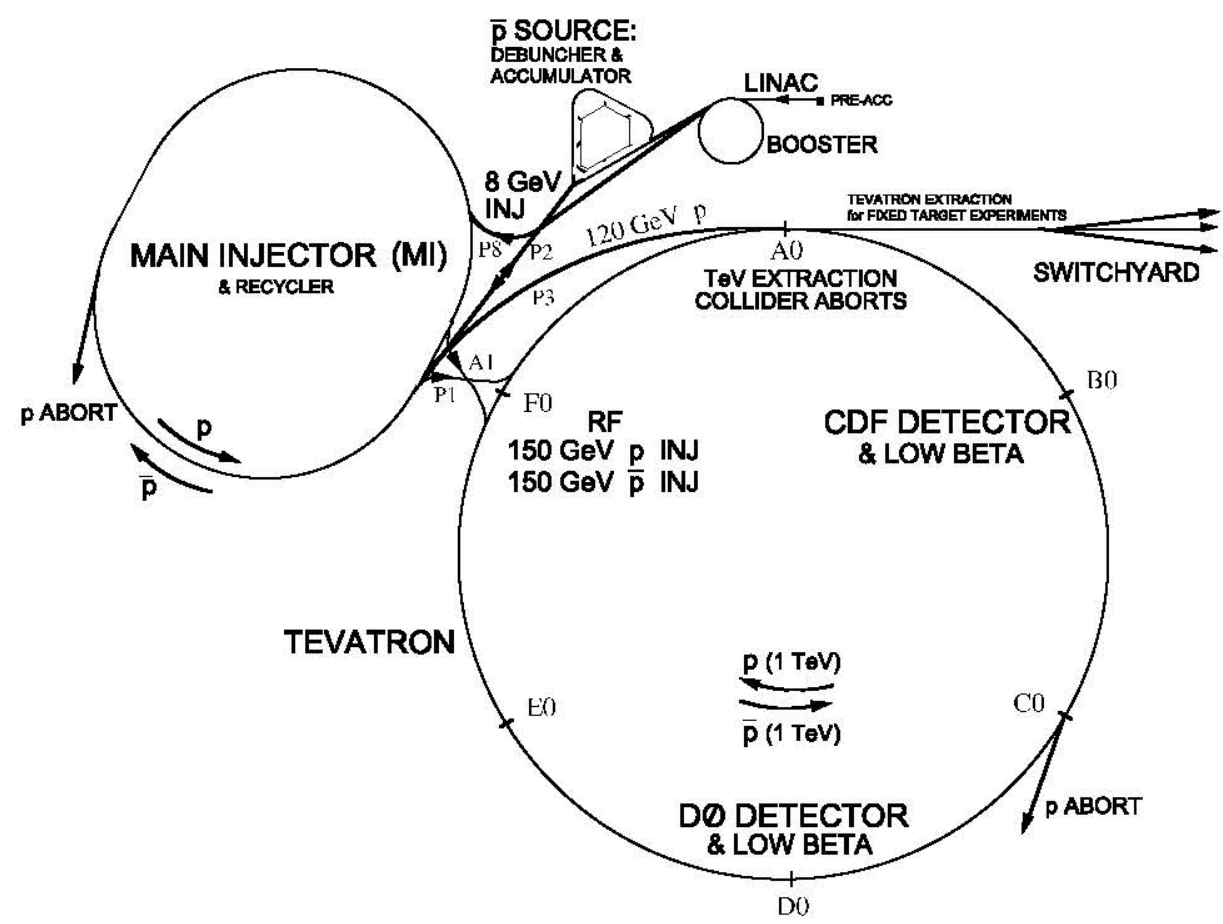

Figure 4.1: A schematic of the Fermi National Accelcrator Laboratory's proton anti-proton collider lacility.

every 1.5 seconds. The incident proton bunches interact with the target protons to yield a proton, anti-proton pair:

$$
p+p \rightarrow p+p+p+\bar{p}+X
$$

apart from a plethora of other products (represented as $X$ in the above equation). The anti-protons produced come out with relativistic energies and in all directions. They are focused into a linear bean with a lithium target acting as a lens[37], then they are sent through a pulsed magnet which acts as a charge-mass spectrometer. Here $8 \mathrm{GeV}$ anti-protons are collected from the spray of particles. The rest of the bearn is then dumped. On average, for every million protons that hit the target, 
only about twenty $8 \mathrm{GeV}$ anti-protons survive to make it to the next stage for further acceleration. Since the incident protons on the nickel target are bunched, the produced anti-protons are bunched too. The Debuncher accelcrator is used to reduce the large energy fluctuations in the beam[36]. These bunches are circulated here until the next component, the Accumulator, is ready to accept a new bunch.

The anti-protons which are circulating and not yet ready to be accepted by the Accumulator are stochastically cooled ${ }^{1}$. The $8 \mathrm{GeV}$ anti-proton beam is extracted from the Accumulator and sent to the VI for subsequent acceleration in a direction opposite to the proton motion as illustrated in Figure 4.1. After the proton and anti-proton bunches reach energies of $150 \mathrm{GeV}$, the beam is directed into the last synchrotron accelerator, the Tevatron.

The Tevatron boosts the proton and anti-proton beam energy from $150 \mathrm{GeV}$ to $980 \mathrm{GcV}$. Numcrous RF cavitics situatcd within the ring produce sinusoidal RF frequency to generate an increasing electric field. As the beam circulates the ring, it is accelcrated to eventually reach $980 \mathrm{GcV}$ cnergy in about 85 scconds. A high magnetic field produced by superconducting electro-magnets constrain the beam within the radius of the ring. For example, in approximately 20 seconds, as the beam energy increases from $150 \mathrm{GeV}$ to $800 \mathrm{GeV}$ after about $10^{6}$ turns around the Tevatron, the magnetic ficld in the Tevatron rises ncarly five fold (from 0.66 Tesla to 3.5 Tesla). On the average the beam gains $650 \mathrm{kcV}$ encrgy from the clectric ficld after each turn. For generation of the high magnetic field there are nearly 1000

\footnotetext{
${ }^{1}$ The anti-protons leave the target at a wide range of energies, positions and angles. This randomness is equivalent to thermodynamic temperature (not physical temperature) so we say that the beam coming off the target is 'hot'. The 'hot' beam will not pass completely into a beam pipe of reasonable dimensions. Also, this hot beam is very diffuse and not intense, or 'bright.' Intense beams are needed in the Collider in order to increase the odds of making a collision produce a rare event. Stochastic cooling is a technique that is used to remove the randomness of the 'hot' beam on a particle-by-particle basis. Simone van der Meer was awarded the Nobel prize for this procedure.
} 
superconducting magnets in the Tevatron, carrying nearly $1 \mathrm{kA}$ of current at low temperatures of about $4 \mathrm{~K}$.

Other than accelerating protons and anti-protons, the 'Tevatron also functions as a storage ring where oppositely moving protons and anti-protons can collide with each other. Once proton and anti-proton beams reach $980 \mathrm{GeV}$ energy the two beams are made to collide at a pre-determined position for hours at a stretch. The operation of generating and circulating the proton and anti- proton beam is called a 'store'. A continuous period of data accumulation during a store is called a 'run'. Fach run is identified by a serial number called the run number. The information obtained from a proton anti-proton collision ('event') during a run is identified via the event number. Once the number of collisions per second (described by the luminosity of the store) decreases to a rate that is too low to be useful for the experiments, the store is ended and the Tevatron is prepared for a new store. For this thesis, collisions are studied at the location called $D \varnothing$ which is shown in the lowest point on the Tevatron ring shown on the schematic in Figure 1.1. The 1) $\varnothing$ detector is housed at this site for our particle physics experiment.

\subsection{The $\mathrm{D} \emptyset$ detector}

The I)Ø Fxperiment[35] is a worldwide collaboration of scientists conducting research on the fundamental nature of matter. The experiment uses the D $\varnothing$ detector for the study and detection of fundamental particles e.g., the $t$ quark, the $W$ and $Z$ bosons, and their interactions, and the search for the IIiggs boson, and even to scarch for clucs to physical phenomenon not represented by the Standard Model.

Bunches of $980 \mathrm{GeV}$ protons collide at the center of the DØ detector with bunches 
of $980 \mathrm{GeV}$ anti-protons coming from the opposite direction. The two independent beams are focused to collide at a point called the beam spot, which is at the center of the detector. This point is the nominal intcraction point.

The proton anti-proton collisions at the Tevatron give rise to a plethora of finalstate particles. These energetic particles interact with the detector material yielding characteristic clues for their identification. Appendix A summarizes the interactions of high energy particles involved in this analysis.

Apart from identification of the particles produced in the proton anti-proton collision, it is essential that the measurement of the positions as well as momenta of these particles be as accurate as possible. In order to do so, we need to define a coordinate system for the detector, which allows us to locate the final position of these particles with respect to one another, as well as with respect to the nominal intcraction point.

\subsubsection{The $D \varnothing$ detector coordinate system}

By convention the direction of the proton beam defines the $+z$ axis of the detector's coordinate system. The origin of the coordinates is defined to be at the nominal interaction point, and a right-handed coordinate system is used. Figure 4.2 is a schematic of the $\mathrm{D} \emptyset$ detector in the $x-y$ coordinate plane.

Since the detector has cylindrical symmetry, it is convenient to use cylindrical polar coordinates for identifying the trajectory of the final-state particles, as well as to locate their final position in the detector. If $x, y$ and $z$ are the coordinates in a rectangular Cartesian coordinate system, the distance from the nominal inter-

action point is $r=\sqrt{x^{2}+y^{2}}$, the azimuthal angle is $\phi=\operatorname{lan}^{-1}\left(\frac{y}{x}\right)$, and for polar 


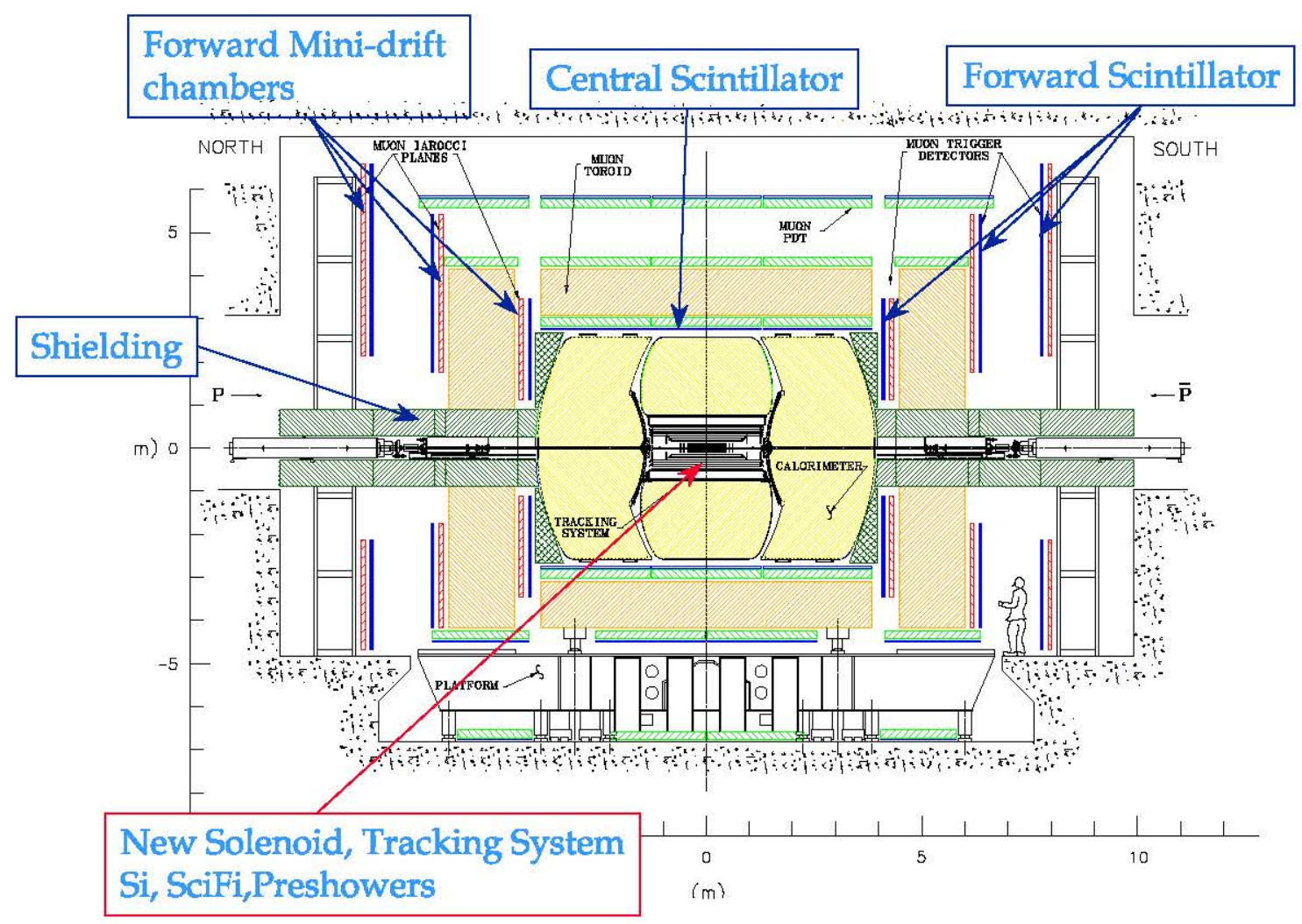

Figure 4.2: A schematic of the $\mathrm{D} \varnothing$ detector in the $x-y$ plane. The direction of the proton beam is from the left to the right and the anti-proton beam is from the opposite dircetion. The upgraded components for Run II are labeled in this plot.

orientation, instead of the angle $\theta$, the pseudo-rapidity variable $\eta$ is used ${ }^{2}$ where

$$
\eta=-\ln \left[\tan \left(\frac{\theta}{2}\right)\right]
$$

IIere, $\eta$ is a convenient choice for polar representation, since the multiplicity of parti-

${ }^{2}$ The rapidity $(y)$, of a particle is defined as

$$
y=\frac{1}{2} \ln \left(\frac{E+p_{z}}{E-p_{z}}\right) .
$$

where $E$ is the energy of the particle and $p_{z}$ is the $z$ component of the momentum of the particle. In the limit that the particle's rest mass cnergy is negligible compared to its total cnergy, we can approximate $y$ by $\eta$. 
cles produced as a function of $\eta$ is roughly unilorm. Fundamentally, the incremental pseudo-rapidity $(\Delta \eta)$ and incremental azimuthal angle $(\Delta \phi)$ are Lorentz invariant quantitics with respect to boosts along the beam direction, and therefore convenicnt for the study of the event topology in the laboratory coordinate system.

\subsubsection{The detector sub-systems}

The DØ detector is a typical multi-component collider detector. It envelops the region around the nominal interaction point. The detector is constructed to extract the maximum information possible about the trajectory of particles produced from the collision and flying outward from the point of interaction. It also provides enough information to enable a measurement of the momentum and in some cases the encrgy of the particles.

Geometrically the detector can be isolated into 3 distinct $\eta$ regions, the central region, the forward and backward regions, and the region between the central and the forward-backward regions, called the inter-cryostat region. The various subsystems are arranged in layers, overlapping symmetrically along the $z$ direction. The $\eta$ ranges of various sub-detectors in these regions are not the same for all, and thus they are able to cover gaps which exist at the boundary of the inter-cryostat region.

The sub-section below is an overview of the $10 \varnothing$ detector sub-systems and a more detailed description is available at[38]. Table 4.1 lists the $\eta$ ranges for the various independent sub-systems.

An event is acceptable if at least one charged particle from the proton anti-proton collision is detected by a pair of Luminosity Monitors within the lime window of consecutive proton and anti-proton bunch crossings. These monitors surround 


\begin{tabular}{|l|l|c|}
\hline functionality & detector & $|\eta|$ range \\
\hline Luminosity & Iuminosity Monitors (L.M) & $2.7<|\eta|<4.4$. \\
\hline Tracker & Silicon Microstrip Tracker (SMT) & $|\eta|<1.62$ \\
\hline & Central Fiber Tracker (CFT) & $|\eta|<1.2$ \\
& Central Prcshower Detector (CPS) & \\
& Forward Preshower Detcetor (FPS) & $1.4<|\eta|<1.6$ \\
& outcr planc FPS & $1.6<|\eta|<2.5$ \\
\hline inner planc FPS & $|\eta|<1.1$ \\
\hline calorimeter & Central Calorimeter (em) & $1.1 \leq|\eta|<1.4$ \\
& Inter-cryostat detector & $1.4 \leq|\eta|<2.4$ \\
& End Calorimeter (em) & $|\eta|<0.7$ \\
& Central Calorimetcr (hadronic) & $1.5 \leq|\eta|<\sim 3.1$ \\
\hline & End Calorimetcr (hadronic) & $0.7 \leq|\eta|<1.5$ \\
\hline Muon & Inter-cryostat detector & $|\eta|<1.6$ \\
& Central Muon System & $1.6<|\eta|<2.0$ \\
\hline Toroid magnct & Forward Muon System & $|\eta|<1.0$ \\
& contral & $1.0<|\eta|<2.5$ \\
\hline
\end{tabular}

Table 4.1: Table of various detector sub-systems and their geometrical acceptance in pseudorapidity.

the beam pipe at $z= \pm 1.35 \mathrm{~cm}$. Listed below are the detector sub-systems, going outward from the interaction point, that a particle produced would cncountcr.

\section{A. Tracking System}

The charged particles which are produced in the proton anti-proton annihilation interact with the components of the tracking system (called tracker for short). If the interactions are recorded by the electronic devices coupled to the detectors, we call the phenomenon a detector hit. Trajectories of the particles are reconstructed by combining the hits obtained from all detector sub-systems. The tracking system along with the magnetic ficld assists identification and the resolution of the tracks left by charged particles. Low momentum particle tracks have a much smaller radius of curvature compared to tracks with high momentum. 
The tracking system can be functionally subdivided into a Si detector, a scintillating detector and a solenoid for producing a magnetic field. The inner-most detector is the Silicon Microstrip Tracker (SMI). This is followed by the Central Fiber Tracker (CFT) which is the scintillating detector. Both the above detectors are immersed in the solenoid's constant magnetic field of 2.0 Tesla which is parallel to the detector's axis. An overall trajectory of particles in flight can be obtaincd using information from the tracker.

\section{i. Silicon microstrip tracker (SMT)}

The SMT detector consists of 6 barrel shaped detectors with silicon (Si) sensors parallel to the $z$ axis. These are closest to the nominal interaction point. There are 12 disk shaped detectors with Si sensors in between and at the end of the barrel segments, these are the $\mathrm{F}$ disks. These lie within the central region of the detector. There are 4 more, larger, disk detectors in the forward region with Si sensors in the transverse plane $(x-y$ plane) called the $\mathrm{H}$ disks. The detector covers a high $\eta$ range, so that it could detect tracks from longitudinally boosted short lived particles, e.g. $B$ hadrons. An added advantage is that it can also detect tracks from primary vertices which may be displaced ${ }^{3}$ from the nominal interaction point by nearly 25 cm.

The Si sensor detectors interact with charged particles produced in the proton anti-proton collision. Figure 1.3 is a schematic of the basic opcration of the detector. The SMT uses $n$-type Si wafers. These silicon wafers, which are 300 micro-meter thin, are probed with very closely spaced, but narrow conducting strips as shown in Figure 4.3. The probe is capacitively coupled (ac coupled) to a p-n semi-conductor junction. A charged particle (with sufficient energy) passing through the Si wafer,

\footnotetext{
${ }^{3}$ The root mean square of the spread in $z$ is $\sim 25 \mathrm{~cm}$.
} 


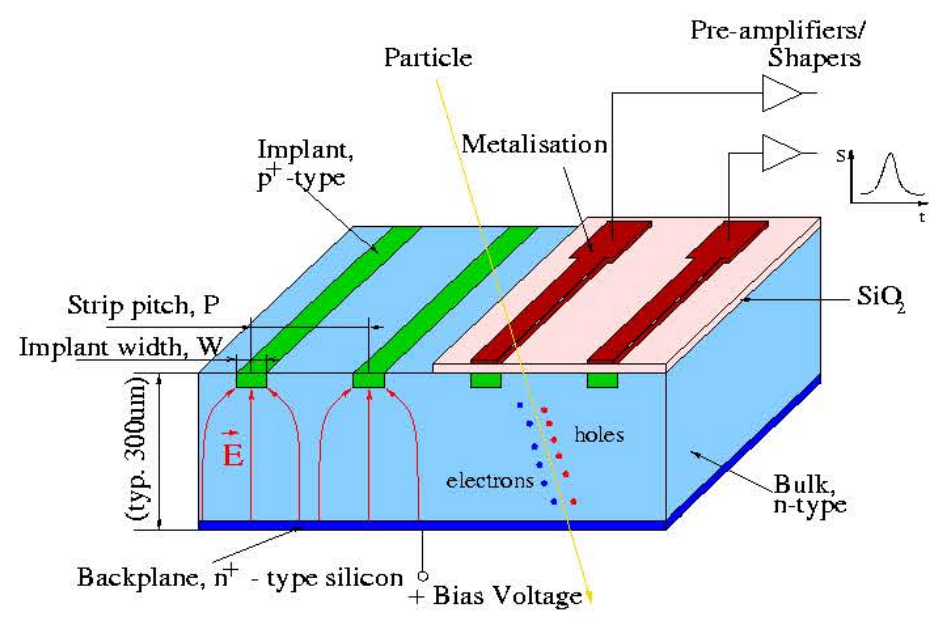

Figure 1.3: A schematic outlining the principles of operation of the unit S.VT detector.

will produce electron-hole pairs in the detector material. The electron-hole current is drawn to the strips by high electric fields. Across the p-n junction, opposite charge is induced on the conducting strips. This charge is then measured. The pair of probes yielding a favorable response indicate the passage of the charged particle within its vicinity. The distance between these strips (pitch of the detector) governs the spatial resolution achieved with the detector.

The barrel's response is used for identifying the trajectory of charged particles (track). $\Lambda$ series of barrel hits are used to depict the track $\eta$ in the ecntral region. They are useful for the identification of $r-\phi$ coordinates of the particles which are detected by the sensors, while the disks measure the $r-\phi$ as well as the $r-z$ coordinates. Due to its position the disk's response is used for the tracks with higher rapidity, or more forward tracks. Using overall information from the hits in the detector a 3 dimensional trajectory of the particles passage within the volume of the subsystem can be reconstructed. 


\section{ii. Central Fiber Tracker (CFT)}

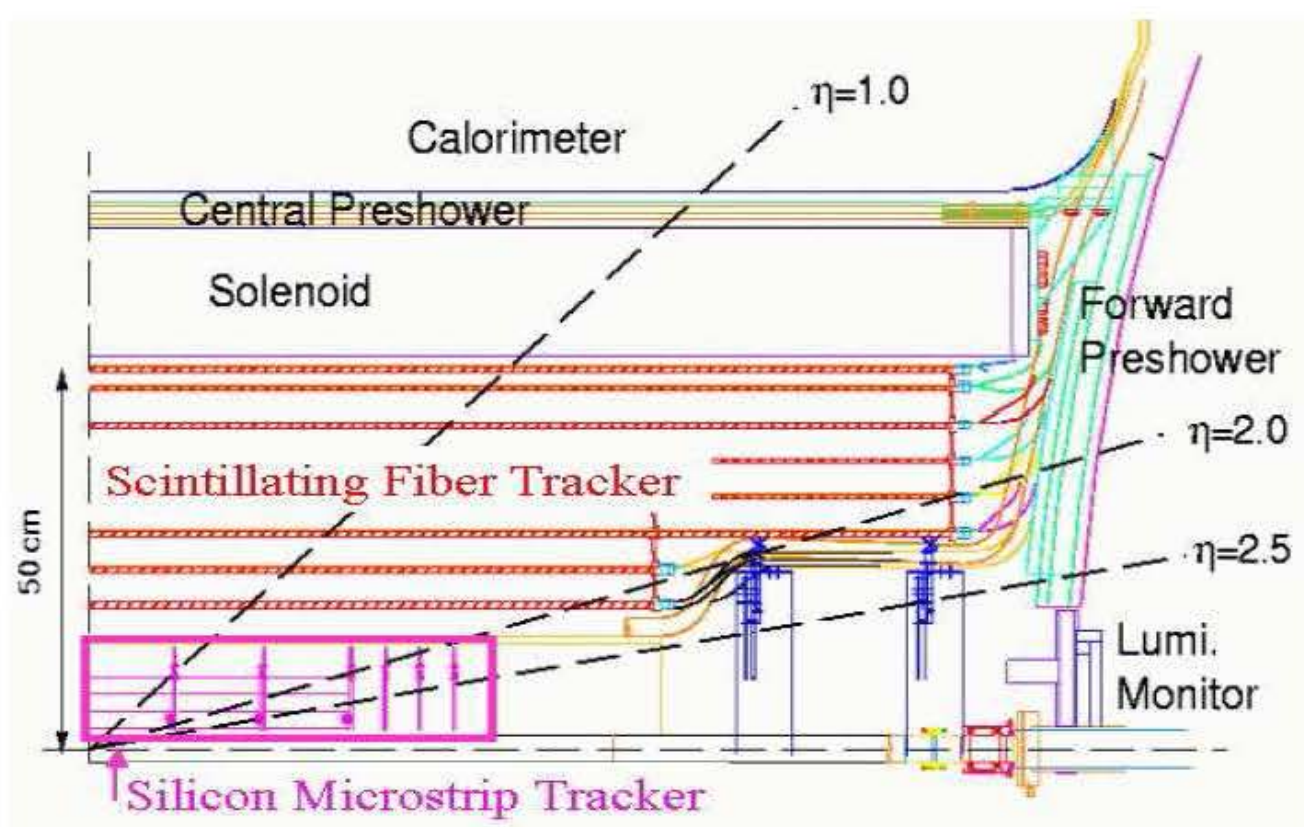

f'igure 1.4: 'Lhe transverse view of the layout of the DQ tracking system. The position of the SHI' and the CFT detectors, with respect to the solenoid housed within the entiral calorimeter core are depicted.

Scintillating fibers are arranged in 8 cylindrical super-layers around the beam pipe. The fibers detect charged particles flying off from the interaction region, and within $|\eta|<1.62$. The response from the fiber trekes is obtaned faster compared to the SMT, and thus the inlonnalion from this system is used to select potentially uschul events (make trigger decisions) from all proton anti-proton collisions. A charged enits photons as it traverses through the scintillating material. 'These photons are transmitted by total internal reflection to the end of the fiber. One end of the fiber is mirrored, and the other ond is optically compled to a wave guide thus enatling the reflected light to propagate via the wave guide to a light measuring device called the visible light photon counter (VIJ) C). This is an avalanche photo 
diode that is operated at liquid He temperatures. The device has a high quantum efficiency $(\sim 80 \%)$ and a high signal gain of over three orders of magnitude. A minimum ionizing particle creates on average cight photo-elcetrons per layer of scintillating fibers. The response from individual fibers in various layers gives useful information about the hits from charged particles.

\section{iii. Solenoid Magnet}

Housed within the central calorimeter's cryostat region, between the CFT and the Preshower detectors, is the supcrconducting solenoid magnet. It produces a magnetic field of 2.0 Tesla uniform in $\eta$ and $\phi$. The Lorentz force bends the trajectory of charged particles. Thus, within the magnetic field, together with the CFT and SMT, a measurement of the track momentum is possible from the measurement of the radius of curvature of the tracks.

The solenoid is designed to present only a small amount of matcrial ${ }^{1}$ to the particles coming from the interaction point, so as to minimize the pair production of photons into $e^{+}, e^{-}$pairs and multiple Coulomb scattering.

\section{B. Preshower detector}

The presence of the solenoid before the electromagnetic calorimeter causes unwanted degradation of the cnergy resolution in the calorimeter. The Preshower detector is meant to make up for the loss in cncrgy resolution, especially for clectrons, by sampling the particle showers dircetly. This is a scintillating detcetor, so neutral particles are undetected by it. The sub-system is split into a central (Central Preshower) and two forward (Forward Preshower) detectors.

\section{i. Central Preshower (CPS) Detector}

The CPS has a $6 \mathrm{~mm}$ lead absorber before the scintillator detectors, to increase

\footnotetext{
${ }^{4}$ It is $\sim 1$ radiation length at $\eta=1$.
} 


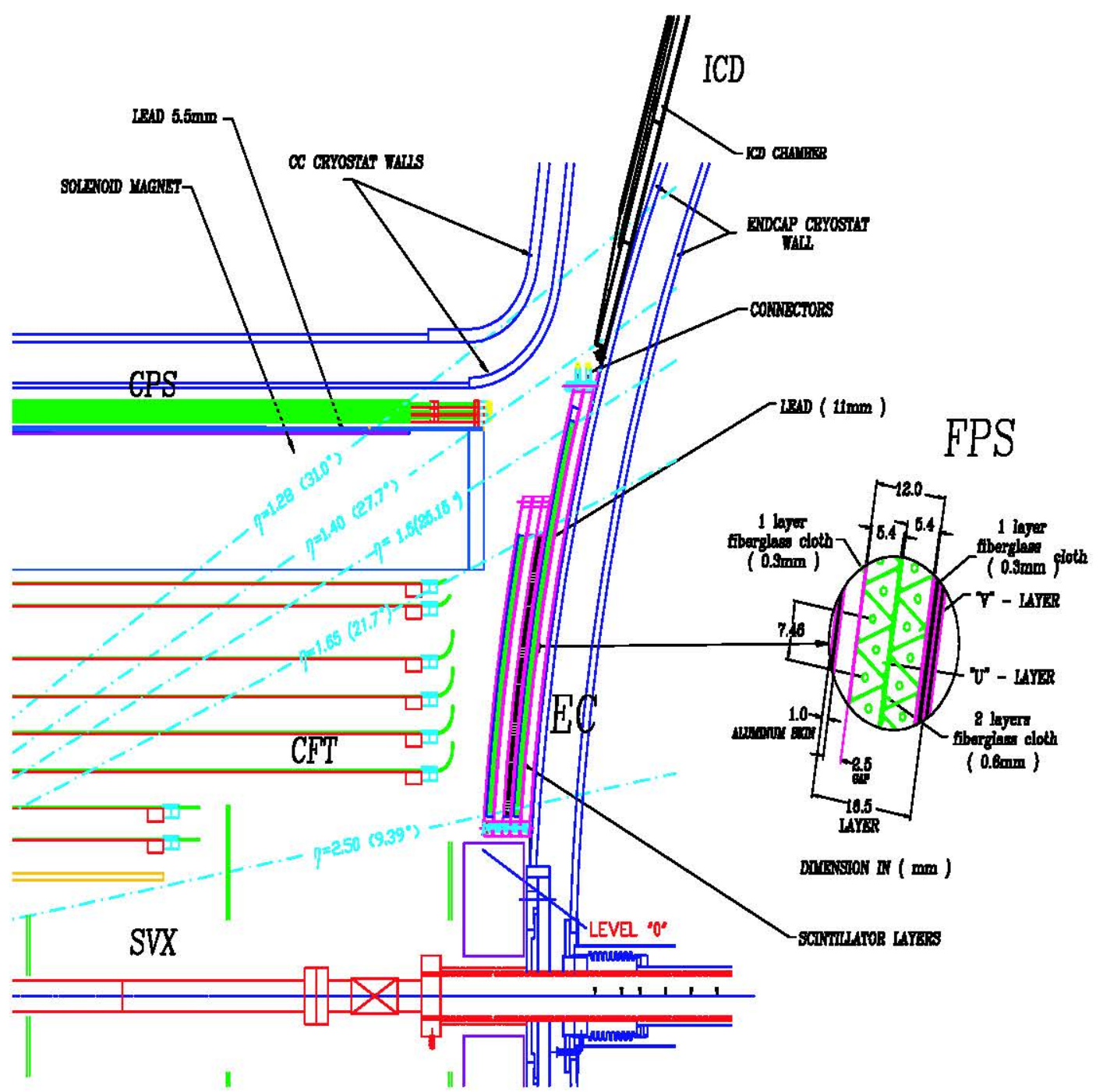

Figure 1.5: A transverse view of one quadrant of the Forward Preshower detector.

the showering of electrons and photons. Threc layers of scintillating strips of triangular cross-section constitute the detector. Each strip has a hole in the center which has a wavelength shifting fiber that directs the light to the waveguides. 'The waveguides transmit the light to the VI,PC similar to that in the CFT.

\section{ii. Forward Preshower}

The forward Preshower detector (FPS) design is similar to the CPS, and has 
similar scintillating strips, execpt that the FPS is mounted in two pieces on the end calorimeters. In the FPS there is a thin $11 \mathrm{~mm}$ lead absorber plate, similar to the CPS. Here, there are two scintillating layers on each side of the absorber. The inner layers detect the minimally ionizing particles e.g., muons, while the outer layers detect the electromagnetic showers which are initiated in the lead plate. The inner layer detector is optimized to mcasure small signals (similar to the CFT), but the outer layer detector is tuned to measure larger signals (similar to the CPS). A particle that initiates a shower in the outcr layer and does not cause scintillation in the inner layers is identified as a photon. However, if it did have scintillation in the inner layer then it is identified as an electron. The role of the FPS is to discriminate between photons and electrons, which is not possible using only the calorimeter.

The spatial resolution for the charged particles from the tracking system is discusscd in Chaptcr 7.

\section{Calorimeter}

The calorimeter detector is designed to identify as well as measure the energy and direction of electrons, photons and hadrons. It is also used in mapping the trajectory of the muons passing through it.

The calorineter is divided into nearly 50,000 cells. These colls are arranged in concentric layers in $\eta-\phi$ space, with the nominal interaction point at the center. In cach laycr, $2 \times 2$ adjacent cclls in $\eta-\phi$ are uniqucly grouped into a Trigger Tower (TT). Analogous to the cells the TTs are also assigned unique integer $\eta$ and

$\phi$ indices to designate their position. For a particular $\eta$ index of the TT, there are 32 'T's covering the $\phi$ space. 'These 'T'T's constitute an $\eta$ ring.

Figure 4.6 represents a quadrant of the $\mathrm{D} \varnothing$ calorimeter in the $x-y$ plane. In terms of their functionality and composition, the calorimeter can be divided into two 
main components, the electromagnetic calorimeter and the hadronic calorimeter. Geometrically, we classify the calorimeter into a central, and two end cap sections. The latter correspond to the forward and backward $\eta$ regions. Each calorimeter cell contains layers of depleted Ur absorber plates sandwiched between I Ar and a resistive plate similar to the one shown in Figure 4.7.

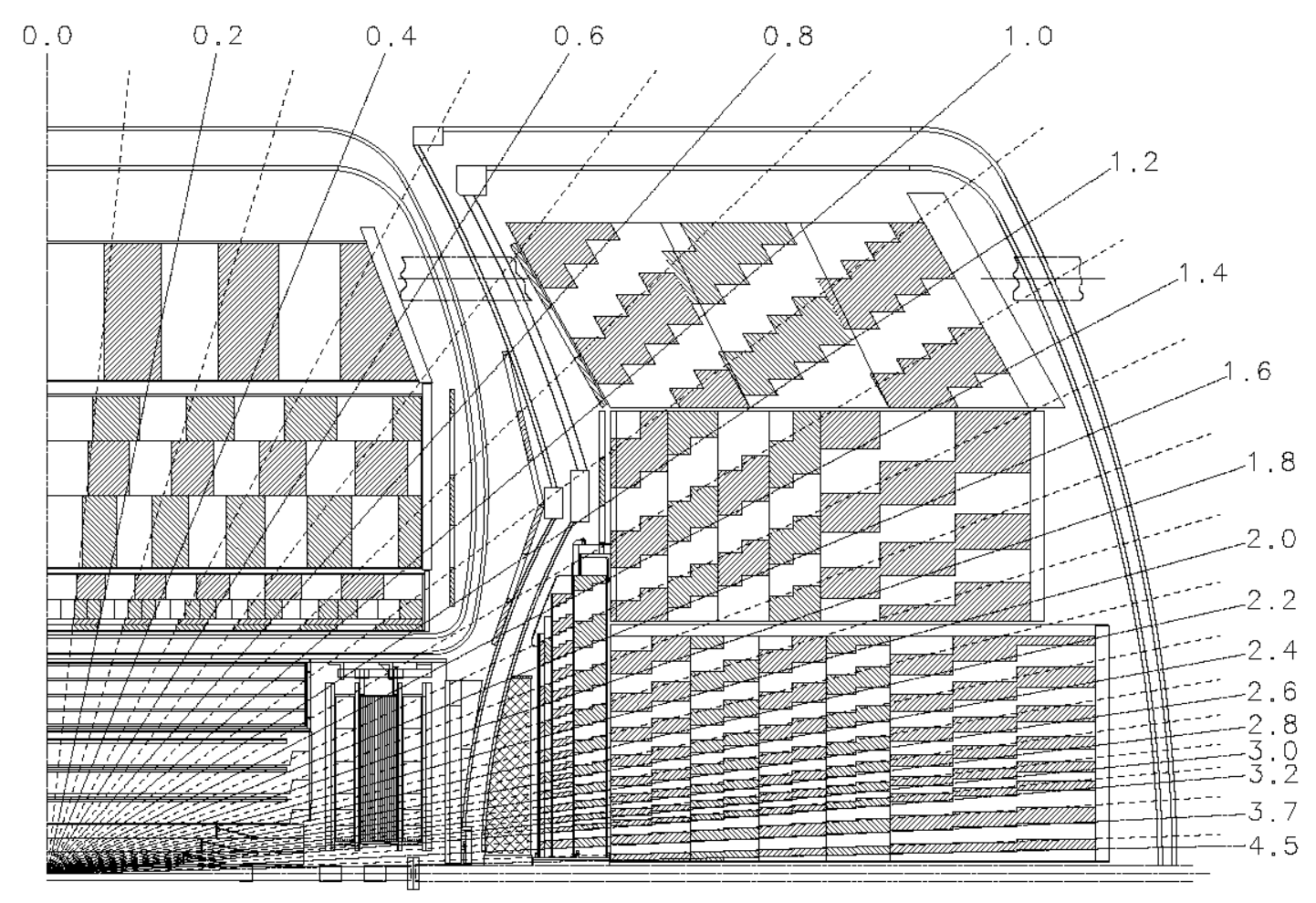

Figure 4.6: $\Lambda$ quadrant of the $\mathrm{D} \varnothing$ calorimeter in the $x-y$ plane.

An incident particle interacts with the Ur absorber producing numerous secondary particles. The secondary particles having sufficient transverse momentum interact with another layer to produce more secondaries. This cumulative eflect leads to a shower of daughter particles. The signal detected is proportional to the number of charged particles traversing the LAr gap (mainly the secondaries). Therefore, the number of secondaries detected in the active material is proportional to the 


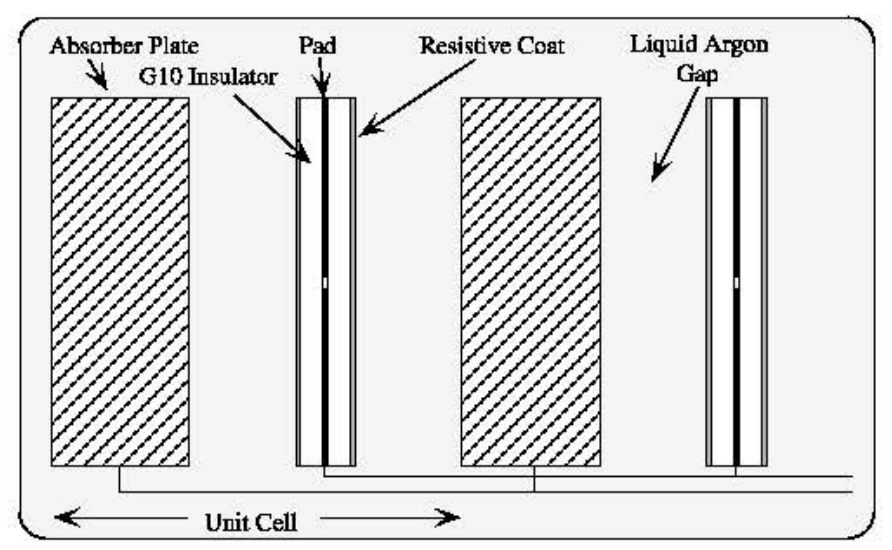

Figure 1.7: Representation of a pair of calorimeter cells, electronically coupled to form a read-out cell of the Do calorimeter.

energy of the incident particle. This is tsed to determine the energy of the incident particle. The drift ficld across the L $\mathrm{Ar}$ gap causes the shower of particles moving in the gap to produce ionization tracks as it moves toward the absorber plate.

The olectrons from ionized Ar drift toward the signal board, producing an electric field that induces a charge in the Cu readout pads. The readout pads for the same $n$ and 9 , but conseculive dephths, are grouped together to form readout cellis. Figure 1.7 is a schematic representation of such a pair of adjacent. readout cells.

\section{i. Electromagnetic calorimeter}

Beginning from the innormost calorimeter layers, 4 layers constitute the EVI calorimeter (FCAI), while the remaining layers constitute the hadronic calorimeter (IICAI). The transverse segmentation of the cells is nearly $0.1 \times 0.1 \eta-\phi$ units, exceps. For layer 3 which is twice ats fine as the other layers. A shower iniliatled by an E.V object would proliferate most in the third layer, and so its granularity is made finer for this layer enhancing the geometric resolution of the showering particles. The EM calorimeter is 21 radiation lengths deep, and this is usually 
sufficient to fully contain shower development of the high encrgy particles which interact electromagnetically with the calorimeter material. The outer layers of the calorimeter constitute the hadronic calorimeter.

The absorber plates are $3 \mathrm{~mm}$ thick in the central calorimeter, and $4 \mathrm{~mm}$ thick in the end calorimeters. Copper pads are sandwiched between circuit boards etched on G10 and thesc pads provide a high clectric ficld (pre-detcrmined as the drift ficld in the LAr active medium) of nearly $2.0-2.5 \mathrm{kV}$ in the $\mathrm{LAr}$ environment.

\section{ii. Hadronic Calorimeter}

Fncompassing the FM calorimeter is the hadronic calorimeter. Functionally, the calorimeter is divided into a fine hadronic (FII) and a coarse hadronic (CII) part, whose energy resolution is much coarser than the former section. Geometrically it comprises of a central and two end calorimeters. The calorimeters are 7 interaction lengths and 9 interaction lengths decp for the central and end calorimeters respectively. Here too, the transverse segmentation of the cells is nearly $0.1 \times 0.1 \eta-\phi$ units, except for cells beyond $|\eta|>3.1$ where the segmentation is twice as coarse. The FH calorimeter consists of $6 \mathrm{~mm}$ uranium-niobium alloy absorber and the $\mathrm{CH}$ calorimeter consists of $46.5 \mathrm{~mm}$ copper absorber plates. Showers of particles produced from hadrons interacting with the detector material develop in these layers.

\section{Muon Detector}

Most of the particles produced are detected and contained after they interact within the calorimeter. Only the neutrinos and high $p_{T}$ muons having a radius of curvature sufficiently large, escape from the calorimeter and into the Muon detector. Muons primarily lose encrgy by ionization when they pass through the bulk of the detector material, producing secondary electrons from the ionized active material. It is reasonable to conjecture that the charged particle which escapes without sub- 
stantial loss of cnergy from the calorimeter sub-system and is detected by the Muon detector is a muon.

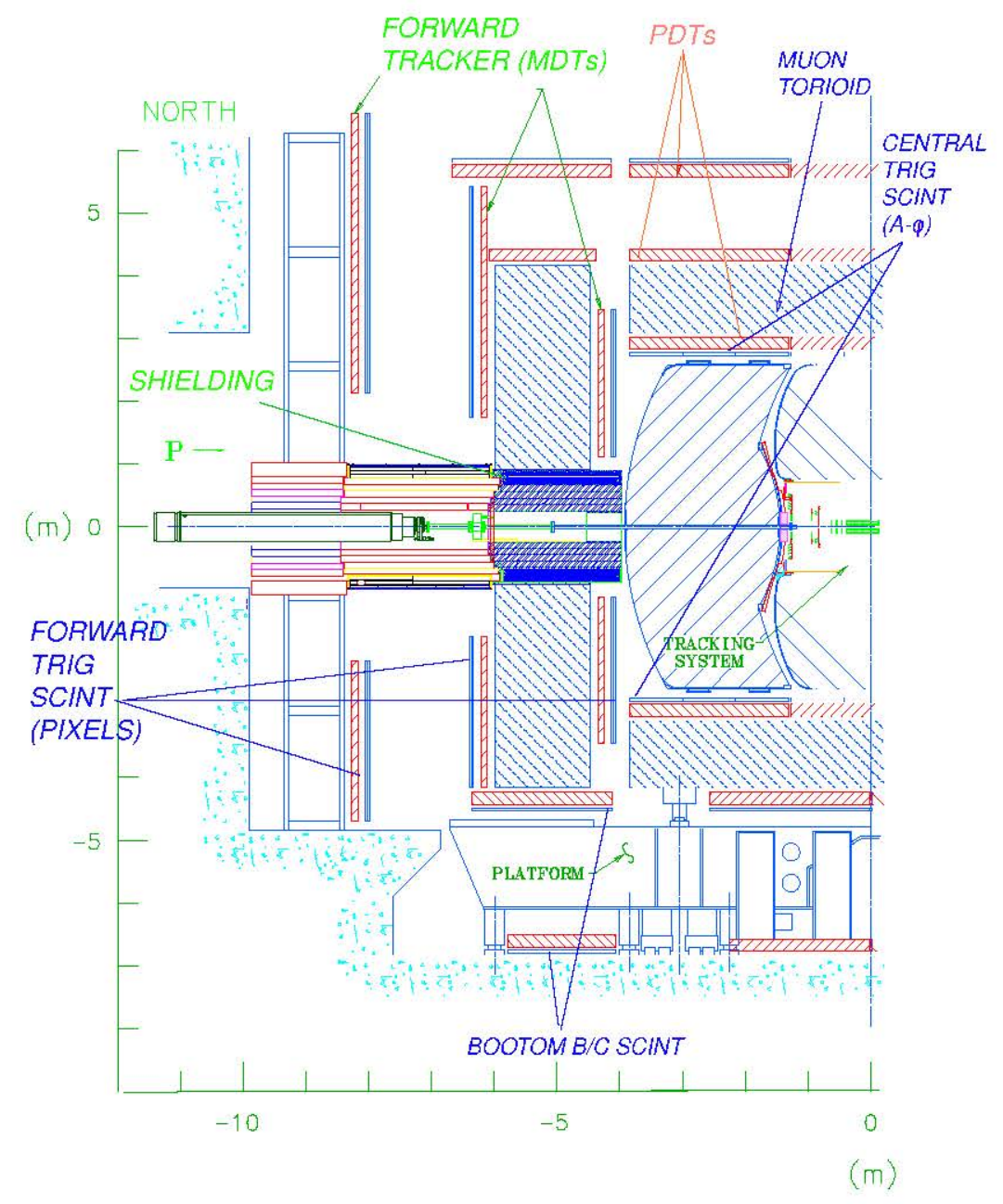

Figure 1.8: The layout of the Muon detector at Dø in the $x-y$ plane. The muon system is housed outside the calorimeter. The Forward and hall of the Central systcms are illustrated.

Like most sub-systems, the Muon detector comprises of threc geometrical sections, a central and two end or forward and backward muon systems. Fach of these is functionally categorized into 3 systems, the A, B and C layer detectors. This gigantic sub-system is the outermost one, and it completcly cnvelopes the calorimeter 
as shown in Figure 1.8. Because ol its cnormous size the detector's sub-systems are spread far apart, and its performance is of coarse granularity. The functional units of the muon detector are single wire proportional chambers (drilt tubes) operating at drift voltages and scintillating fibers. The proportional drift tubes (PI)Ts) are confined to the central region, but in the forward system they are replaced by drift tubes called mini drift tubes (MDTs). Scintillating detectors are used in both the central as well as forward regions.

Muons passing through the drift tubes ionize the gas it contains. The secondary electrons which are produced accelerate under the influence of the constant electric field toward the central anode wire as well as the charged anode pads on the periphery of the drift tubes. They cause further ionization of the gas in the drift tubes, leading to production of more electron ion pairs, subsequently leading to an avalanche in clectron production in the neighbourhood of the anode. The ions, which are much more massive, drift away from the anode making way for the avalance electrons. As they move towards the cathode, they induce an opposite charge on the cathode. From the delay in the response of the avalanche electrons reaching the anode wire and the anode pad, the position of the initial interaction of the muon can be estimated. Neighboring drift tubes are staggered in alignment, so that the position of the muon's passage in the detector is obtaincd as it passes through it, and hence its passage as a function of time is deterministic.

The muon system has three large toroid magnets, one central and one each in the forward-backward regions. The Lorentz force due to the magnetic field causes the muon to curve. After determining the radius of eurvature of the trajectory between the $\mathrm{A}, \mathrm{B}$ and $\mathrm{C}$ layers it is possible to determine the $p$ of the muon track.

The resolution of individual hits obtained from the detector sub-system, the 
magnetic ficld strength and the total number of hits obtained as the particle moves through the detector, are the primary contributions to the overall position resolution of the particle track. 


\section{Chapter 5}

\section{Simulations}

This chapter describes the generation of simulated events which are used in the analysis. The data events of interest are rare, therefore understanding the physical obscrvables involves $11 \mathrm{se}$ of computer-based Montc Carlo (MC) methods for simulating many such events. Moreover, in order to plan the system of detectors, we need to study the simulations of a wide variety of processes which could be of potcntial interest. Simulations cnable budget estimation and planming as woll.

Simulations help us understand the interaction of high energy particles with the detector, and also help determine the geometric acceptanec, the resolution and the efficiency of our detectors. However, accurate simulation warrants the knowledge of the plysical interactions of the particles with the detector material.

Simulated events from signal as well as background processes which have a worthy representation of data sets are widely used for obtaining an optimal set of selection critcrion. Although the relative normalization between signal and background is estimated using data, these normalizations depend on the purity of the selected data cnscmble. The aim is always to kecp the purity of the cnscmble as high as possible, and to minimize the losses in signal events as a result of the selcetion 
critcrion, i.e. selcction cuts ${ }^{1}$.

There are two fundamental steps involved in the generation of a Monte Carlo simulated event. It first involves the gencration of the particles produced in a specific physics process, and secondly a simulation of the interaction of the final-state particles within the detector.

\subsection{An overview}

'This section deals with the simulation of an event which cvolves from a proton anti-proton collision. These generators simulate specific physics processes using computer generated pseudo-random numbers, utilizing known cross-sections for their production. Various steps are involved in this process. Figure 5.1 illustrates diagrammatically the various steps which occur during typical event generation. Using the parton density functions (proton as well as the anti-proton) the hard scatter final-states are first produced. Then using the showering and hadronization generators, a list of the final state particles in the event are produced. The list includes the identities as well as all kinematic information of the particles. Primarily, a simulated physics event consisting of all final-state particles is generated using an event gencrator. Then the underlying interactions are simulated giving rise to physical particles using a showering and hadronization generator. Iastly, the interaction of the final-state particles with the various sub-detectors is simulated incorporating realistic effects, e.g. presence of a magnetic field in the tracking region, and detector resolutions.

The validity of the simulation is tested in regions of kinematic phase space where

\footnotetext{
${ }^{1}$ This is deseribed in the Appendix D.
} 


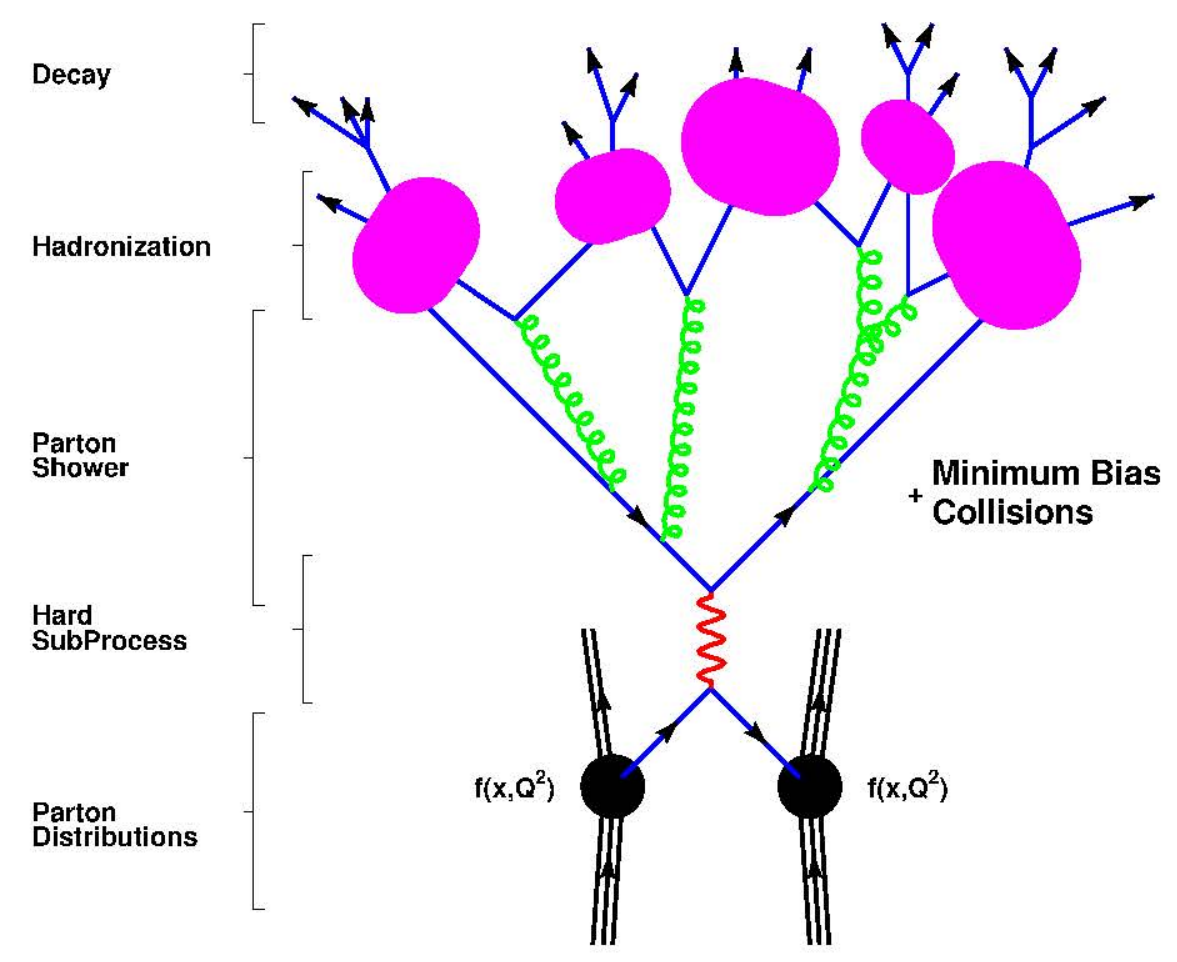

Figure 5.1: Schematic illustration of the sequences in a generic event generator starting from the proton anti-proton hard scatter interaction. This diagram illustrates the parton shower in the final-state, however one can have initial-state parton showering too. The time axis points vertically upward. This figure is obtained from [41].

the detector acceptance is high. Distributions of physical observables from data are compared with those from simulated cvents. The resemblance of the two distributions constitute a benchmark for the success of cvent simulation. In cases of rare events, or unobserved phenomena, the simulated distributions only mimic theoretical predictions used in modeling them. If in addition, for a physical observable, an extrapolation to unmeasured regions in phase space is desired, then a prediction of the differential cross-section in that region is utilized. One such example is that of the limited solid-angle coverage due to holes or cracks in the detector.

Some essential ingredients for event simulation are summarized here. 


\section{A. parton distribution functions}

The measurement of the $t \bar{t}$ cross-section relies upon the knowledge of the probability distribution of the momentum fraction $x$ of the partons in a proton (or antiproton), at a particular value of momentum transfer. This is the parton density function of the parton in the proton (or anti-proton). The parton density function is determined experimentally. Once the cross-scction is known then the all-inclusive physics processes can be simulated in ratios which are in agreement with measurements.

The MC signal events which have been produced are using the CTEQ6.1 M parton distribution functions[42]. These distributions have been established by the CTEQ collaboration[42]. Figure 5.2 illustrates the CTFQ6.1 distributions for some partons as a function of high momentum transfer $Q^{2}$ value.

\section{B. Leading order matrix element generators}

Figure 5.1 illustrates the basic principle of the $2 \rightarrow 2$ hard scatter process where two partons from the incoming proton and anti-proton interact giving rise to two new partons, while the non-interacting partons constitute the remnants. Once the hard scatter process is determined, theoretical principles are used to compute the matrix clements of interactions where there are a fixed number of particles in the final-state. The mathematical degrec of complexity grows with the increase in number of final state particles.

Typically an event generator provides a list of simulated particles simultaneously seen in the detector from an event. Every particle's identity, and 4-momentum is known. In addition, the initial position or vertex information may also be saved in the list.

For this analysis, the hadronic collisions which are well described within the 


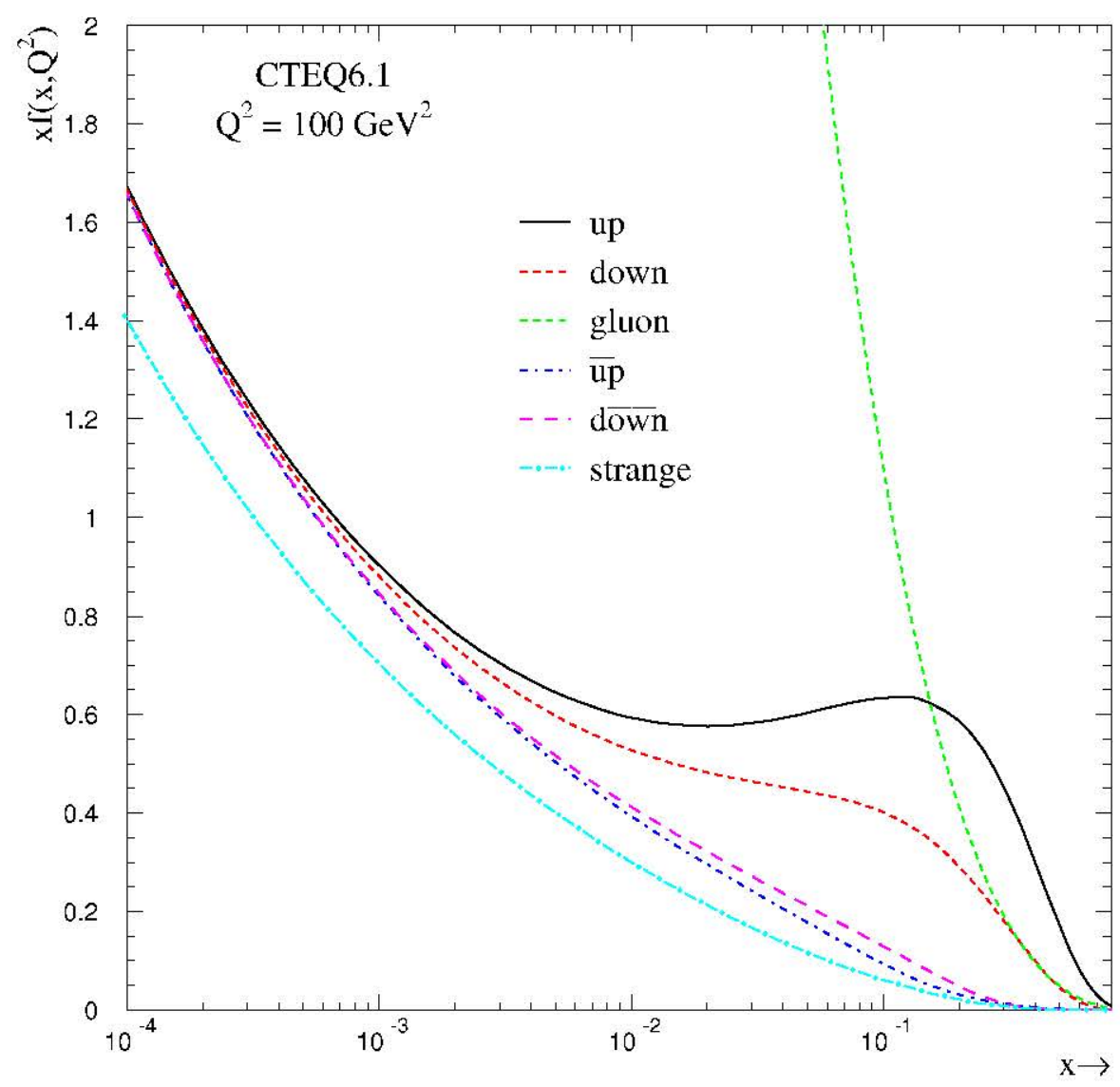

Figure 5.2: The proton's parton distribution functions from the CTEQ6.1 set plotted at a $Q^{2}$ value of $100 \mathrm{GeV}$. This figure is obtained from [41].

framework of the Standard Model are simulated. For initiating the hard scatter, the signal and background processes for the analysis are generated at $\sqrt{s}=1.96 \mathrm{TcV}$ using the Alpgen[43] Monte Carlo generator, version 1.2.

The Alpgen generator is based on exact leading order evaluation of parton matrix clements, which include the $t$ and $b$ quark masses. In specific cases the $c$ quark mass may also be included. Starting from a 2 parton initial-state, up to 6 final state partons can be accommodated. This leads to the estimation of matrix clements for the signal as well as background production process which may or may not have 


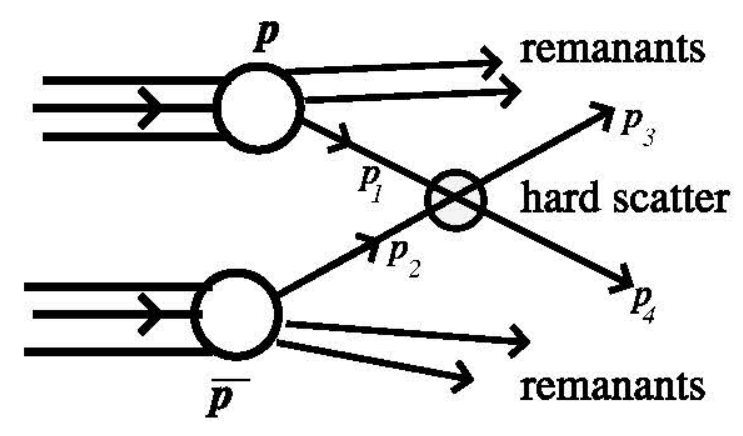

Figure 5.3: A schematic showing the $2 \rightarrow 2$ scattering process for a proton antiproton collision. Two partons coming from the proton and anti-proton carry only a fraction of the proton and anti-proton momentum. The remaining fractions remain with the other non-interacting partons. Incoming partons have 4-momentum denoted by $p_{1}, p_{2}$ while the out going partons have 4 -momentum denoted by $p_{3}, p_{4}$.

associated initial-state radiation and final-state radiation.

\section{Higher order corrections: perturbative and non-perturbative QCD computations}

Interactions mediated by real and virtual bosons are described as well. Real gauge boson emission diagrams are considered in the context of perturbative computation. The real emission diagrams are based on the leading order matrix element generators, and can be evaluated. Virtual particles that may possibly be emitted or absorbed are also included in calculations. However as one proceeds to calculate from one order to the next, the mathernatical complexity increases.

There are two traditional approaches to model these higher order processes. In one of the methods the matrix element corresponding to the process is calculated order by order. These describe the initial-state radiation and final-state radiation states as well. Since the phase space available for glnon cmission increases with energy, the estimation of matrix-element becomes less relevant for the full reconstruction of events at higher energies. At high cnergies the perturbative cxpansion is feasible since the coupling strength at these scales are much smaller compared to 
unity, and this is done in the sccond method.

\section{Showering and hadronization event generators}

The QCD perturbative theory holds well at short distance scales $\left(\sim 10^{-18} \mathrm{~m}\right)$. At large distance scales the interaction strength (coupling constant) increases and a perturbative approach breaks down. At these scales the partons are incorporated as bound states. This takes place via the fragmentation process and then the hadronization process.

The fragmentation process is not well understood from first principles, i.e. from the QCI) I agrangian. There are three popular computational models which attempt to simulate this phenomenon. These models are the string fragmentation model, the cluster fragmentation model and independent fragmentation model. The success of the models is judged in terms of how well they mimic the data from the Tevatron.

There are tools in the form of computer programs which model the showering and hadronization of the free particle final-state products. These are the showcring and hadronization event generators.Partons produced in the event undergo fragmentation thus allowing the quarks to branch into $(q, g)$ pairs, anti-quarks into $(\bar{q}, g)$ pairs, and the gluons into $(g, g)$ or $(q, \bar{q})$ pairs. The fragmented partons are hadronized employing various hadronization models.

Pythia[44] uses the Lund String fragmentation and hadronization scheme. Another SHG, Isajet[16] uscs the Feynman-Ficld scheme. Herwig[15] uses the cluster fragmentation scheme. In this analysis we use Pythia[44] version 6.2(CTEQ5L) for simulating the fragmentation and hadronization. EvtGen[47] is used to model the decays of the $b$ hadrons to their final-states. The last step in the event generation is to evolve and hadronize spectator partons, i.e. those partons which have not formed physical states with other partons in the event. 'There is no unique way to incor- 
porate these lelt over partons. Pythia uses an extension of the Lund Color scheme while Isajet overlays minimum bias events over the primary hard scatter event.

\subsection{Simulation of the physics processes}

The hard scatter process used for the generation of simulated data is $t \bar{t} \rightarrow b b$ $l_{1}^{+} l_{2}^{-} \nu_{l_{1}^{-}} \nu_{l_{2}^{-}}$and these are generated for 7 diflerent input values of the $l$ quark mass viz. 120,140,160,175,190,210,230 GeV. The samples have contributions of tau lepton states decaying into hadronic as woll as leptonic channcls. However, the di-electron channel signal process is:

$$
p \bar{p} \rightarrow t \bar{t}+X \rightarrow e^{+} e^{-} b b \overline{\nu_{e} \nu_{\epsilon}}+X
$$

while that for the di-muon channel is:

$$
p \bar{p} \rightarrow t \bar{t}+X \rightarrow \mu^{+} \mu^{-} b \bar{b} \overline{\nu_{\mu}} \nu_{\mu}+X
$$

The $e \mu$ channel processes are:

$$
p \bar{p} \rightarrow t t+X \rightarrow e^{+} \mu^{-} b \bar{b} \nu_{e} \bar{\nu}_{\mu}+X
$$

as wcll as

$$
p \bar{p} \rightarrow t \bar{t}+X \rightarrow \mu^{+} e^{-} b b \nu_{\mu} \bar{\nu}_{e}+X
$$

It is also possible that the response from final-state objects can be laked by processes other than those mentioned above. These constitute the background processes. The principal background process in the analysis is $Z / \gamma^{*} \rightarrow l_{1}^{+} l_{1}^{-}+j j$, where $l$ indicates 
$e, \mu$, or $\tau$ lepton. The di-boson process $W^{+} W^{-} \rightarrow l_{1}^{+} l_{2}^{-}+j j$ is also a background process. Simulated events corresponding to signal and background processes were gencrated using Alpgen followed by Pythia. Details of the generation of specific processes are given in [51] and [52].

\subsection{Simulation of the $\mathrm{D} \emptyset$ detector}

The Detector Description and Simulation Tool, also known as GFA.NT[48], is a progran that describes the passage of elementary particles through a varicty of materials of different shapes and sizes. For instance consider the fabrication of the vertex tracking detector. This detector, being closest to the nominal interaction point, is prone to extensive radiation damagc. If we use a detcctor which is made of Si, e.g. our current SMT detector, then the typical life-time of the material before which it is considered damaged due to radiation is nearly $2 \mathrm{fb}^{-1}$ of integrated luminosity ${ }^{2}[39]$. However, if the exact same detector design is used but the silicon material is replaced with artificially produced diamond, then the lifetime of the detector is increased[10]. This is however an cxpensive choice. Simulating various detector geometry, an optimal design can be achieved using less expensive material. Therefore, before building an actual detector, a complete simulation of the experiment helps in considering the benefits and optimal utility of the detector over the costs and the time required for the construction.

Moreover final-state products produced in the detector interact with the detector material and the eventual resolution with which we measure the physical quantities is unrealistic. This is a uscful tool for studying the responses from physics objects

\footnotetext{
${ }^{2}$ From tests done with the Rum II design specifications.
} 
with realistic detector effects and resolutions which match that obtained from data. The full simulation path consists of two programs: DØgstar[49] and DØsim[50]. This section highlights the simulation of the $\mathrm{D} \emptyset$ detector's response.

DØGFANT Simulation of the Total Apparatus Response (I)Øgstar)[49] is a simulation package (or program) which is available for the generation of Monte Carlo studies of the $\mathrm{D} \varnothing$ detector with different configurations, e.g. with the magnetic field in the tracking system set off, or even if its polarity were changed. It provides uscrs with a full GEANT simulation of all the various sub-detectors with a simple interface. After that, information can be simulated at the basic level of electronic channels, e.g. studies with some disabled SMT detector channels can also be performed and the effects on identifying and diagnosing simulated events can be done as well.

DOgstar is a wrapper for GEANT. It determines the amount of energy deposition in the active region of the detector. The primary sequences of the DØgeant program arc:

- DØgen: which is the standard event generation package,

- 1) Øgeo: which creates the GFA.NT geometry parameters,

- I) Økin: which is a package which deals with kinematics for I) Øgstar,

The DØSim package is used to perform the electronics simulation and pilcup of any additional minimum bias interactions that occur in the same bunch crossing as the signal event. It is used to gencrate files suitable as input for the reconstruction software (DØreco) starting from files supplied by DØgstar program. The analog output of DØgstar is digitized for each detector at this stage. The various steps are:

- morge hard scatter and minimum bias events 
- add calorimeter pileup from previous evcuts

- make I,1 calorimeter trigger tower information for L,1 simulation

- add calorimeter noise

- add SMT noise and inefficiencies

- add CFT noise and inefficiencies

- add Muon noise and inefficiencies

- save all relevant kinematic information from events

\subsection{Additional corrections on simulated events}

Due to our lack of complete understanding of the detector deficiencies, additional corrections are applicd to fully simulated and reconstructed events so as to match the response from data. For example, there is an additional correction factor applied to the efficiency per muon in every object derived by E. Varnes[53]. The oversmearing of missing transverse cnergy in $Z \rightarrow e e+X$ Monte Carlo cvents from $\Lambda$. Kumar, et. al.[54] is also applied. The over-smearing corrections are described in Chapter 7. 


\section{Chapter 6}

\section{Data Selection}

Not every proton anti-proton collision is useful for the physics goal of this thesis. Events of interest have to be sorted from a large number of events. Only a couple of relcvant cvents are cxpceted from over $10^{10}$ proton and anti-proton collisions. This chapter describes how potentially useful events are selected from all proton anti-proton collisions. The selected events constitute the data ensemble.

\subsection{Event signature}

From the SM we can inler that the production and decay vertex of $l$ quark are separated by $\sim 10^{-16} \mathrm{~m}$, which is smaller than the spatial resolution of our detectors, therefore inhibiting the direct detection of the $t$ quark. So its detection proceeds through the identification and reconstruction of all its decay products.

The large mass of the $t$ quark restricts it from being produced with high relativistic momentum. It decays into the $b$ quark and the $W$ boson. In the di-lepton channel, the $W$ boson subsequently decays into $e$ and $\nu_{e}$, or $\mu$ and $\nu_{\mu}$. Therefore, these lighter decay products have high momentum and large angular separation in 


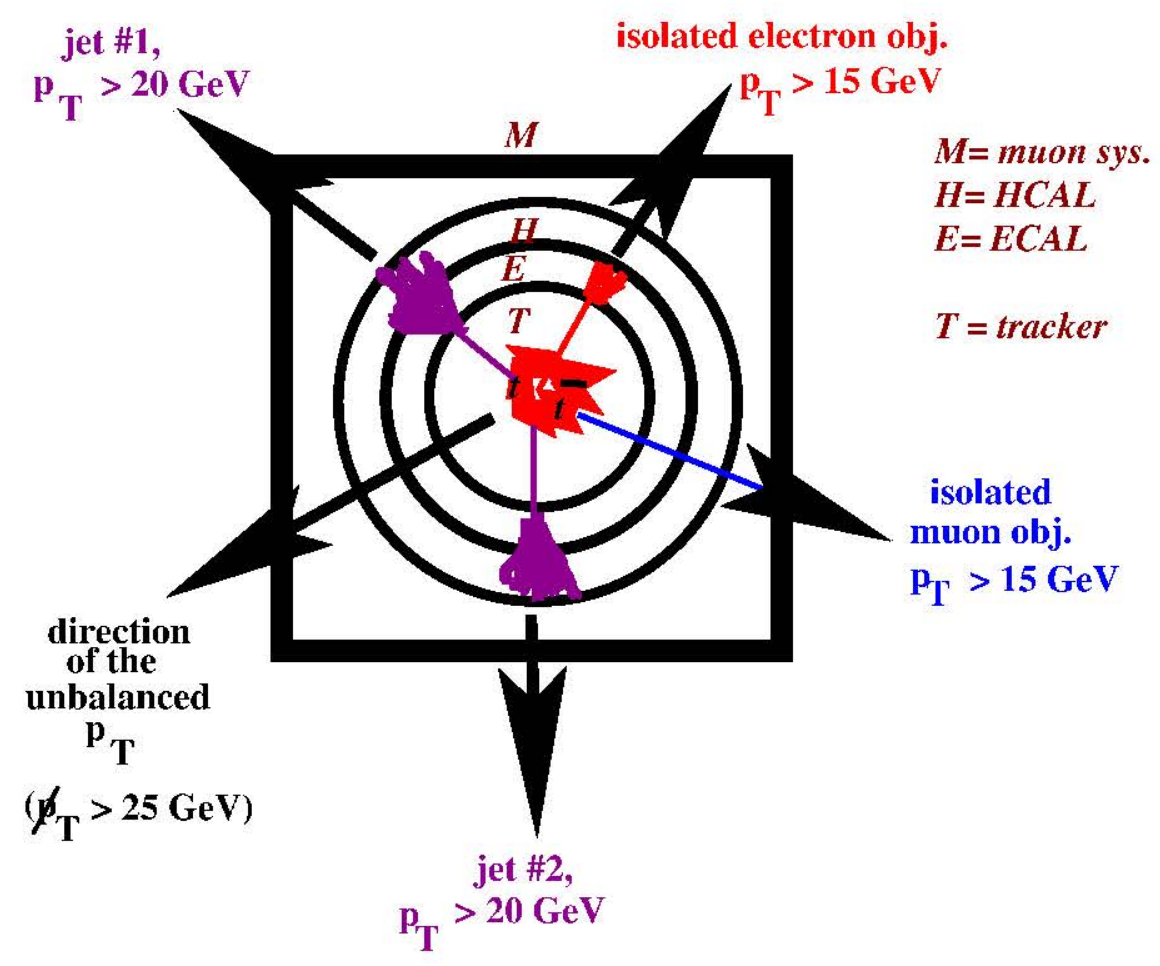

Figure 6.1: A sketch representing the signature of a typical $e \mu$ candidate event within the detcetor.

the laboratory frame of reference. This ensures that on the average, the stable decay products have a high transverse momentum $\left(p_{T}\right)$, and are isolated with respect to one another. The final products detected are the jets from the $b$-quark, and the two charged leptons. The neutrinos remain undetected. Figure 6.1 is a cartoon of the characteristic event signature of an $e \mu$ event. Summarizing, we have the event signature as one with at least:

1. Two ${ }^{1}$ high $p_{T}$ isolated jet objects.

2. Isolated high $p_{T}$ electron positron pair in the, di-electron channel, isolated high $p_{T}$ muon anti-muon pair in the, di-muon channel,

\footnotetext{
${ }^{1}$ There can be more than two jet objects in the event, and it may be attributed to initial-statie radiatio or final-state radiation.
} 


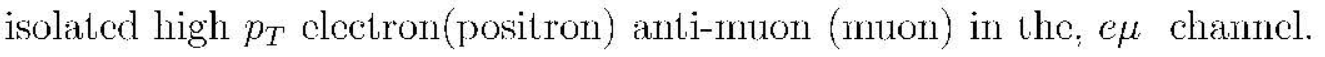

3. Large imbalance in the transverse nomentum duc to the undetected neutrinos.

The event signature can be laked by some non-top quark processes as well. These processes are:

1. The $Z$ boson production accompanied by at least 2 hadronic jet objects, and where the $Z$-boson may decay into a pair of oppositely charged, but same flavor leptons. This process is the primary physics process which mimics the event signature in the di-electron and the di-muon channels. When the $Z$ boson decays into a pair of $\tau$ leptons, and they decay into $e$ and a $\mu$ then it is possible to fake the $e \mu$ channel characteristic as well. Here the mis-measurement or resolution effects contribute to the imbalance in the transverse momentum of the original event.

2. The di-boson $W^{+} W^{-}$production, once again accompanied with the production of at least 2 hadronic jets, is also a source of a physics process faking the dilepton decay channel. The $W$ boson decays into the charged lepton and its corresponding neutrino. Along with the hadronic jets, this process mimics the cvent signature as well.

3. The detcetor resolution effects contribute to a class of fake cvents called instrumental fakes. Consider an event final-state which has a muon and at least 3 jet objects. A jet object can mimic an electron object when it has sufficient electro-magnetic energy contribution in the calorimeter. In such a case the e $\mu$ event can be faked. IIowever, in the above scenario, if there were an electron object and at least 3 jets, instead of a muon object, then a di-clectron object can be faked instcad. 


\subsection{The strategy of event selection}

All sub-systems of the 1)Ø detector are used to identify the objects produced in an event. From the detected final-state products, the puzzle of inferring the initial physics process is solved.

Information from an event is not available immediately after a plysics collision. In fact much of the information is available later, and therefore event selection is achieved only via a carefully designed selection scheme which filters out unwanted events in stages.

The following sub-sections describe the systematic process in which useful events are identified, and associated information is saved. Our resources limit the amount of information we can save. We cannot record information from all collisions because they occur too frequently, even before the previous event is recorded. Moreover, if we were in a hypothetical position to record every event, then we would not be able to reconstruct all of it and save thern on tape devices in a reasonable time. Filtering

of the events at $D \varnothing$ is achieved in three stages by using a trigger system. The purpose of the trigger system is to produce a signal that starts the readout of the events at the appropriate stage. It is desirable to record and save all useful proton anti-proton collisions and reduce the background events.

Figure 6.2 is a flowchart of the tri-level trigger system and data accumulation at DØ. The detector readout electronics design allows us to save about $10^{4}$ events per second at the first stage called the level one (I.1) trigger system. Here, the decision whether or not to read out all detector elements is taken. At this stage electronic information which can be read out fast from detcetors is utilized for estimating the importance of the event. If the decision is not to take the event, the readout 


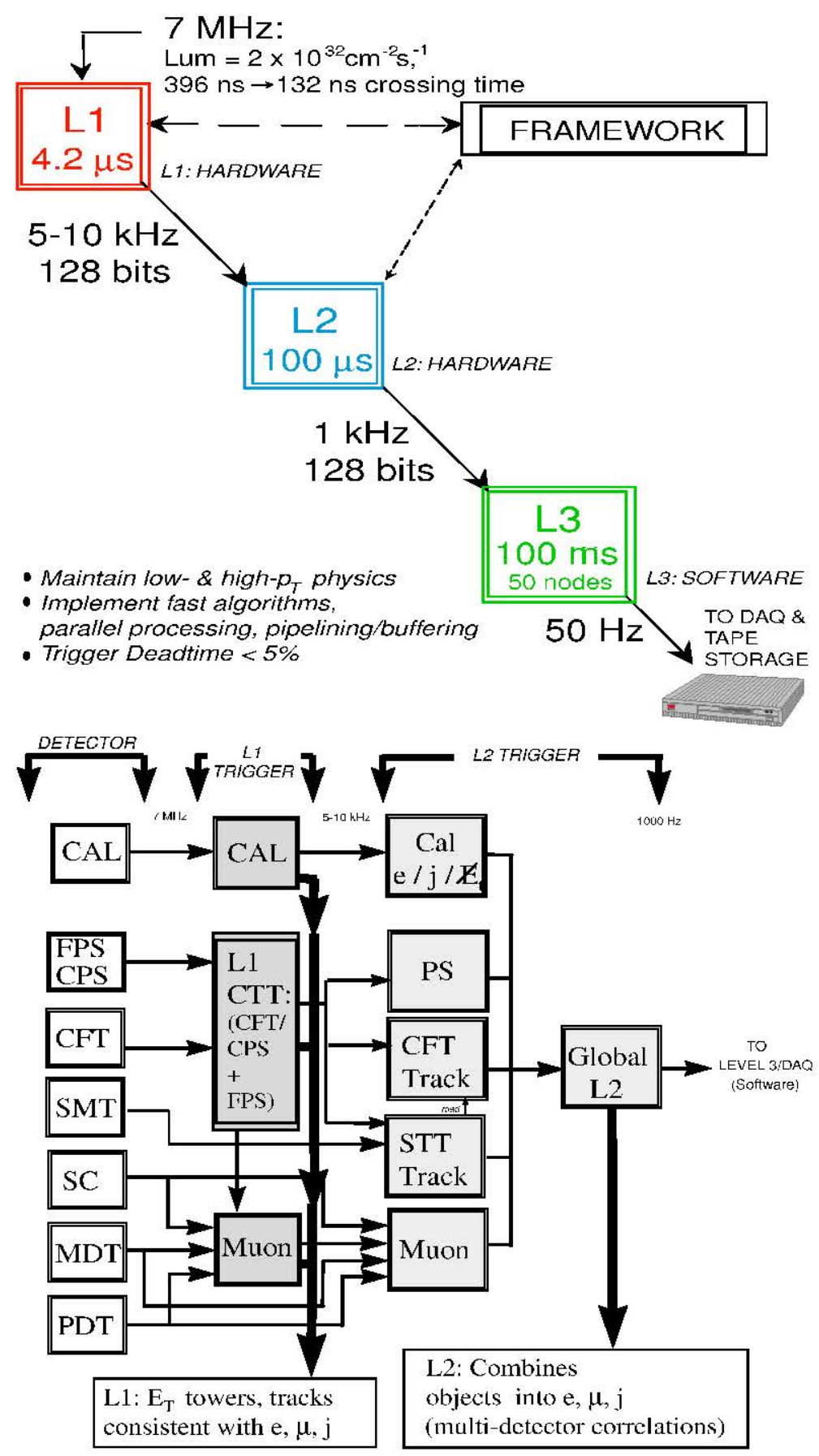

Figure 6.2: (Top) Summary of the three level I) $\varnothing$ trigger system in Run II. The allocated bandwidth and decision time are indicated in the schematic.

(Bottom) The flowchart of L1 and L2 triggered data path. The arrow indicates the dircction of data flow. 
clectronics is kept ready for the next cvent. Characteristic, but coarse information from the calorimeter and muon detector is utilized for accessing the importance of the event. 'The trigger decision at L1 is on-line, which indicates that the decision to record the event is taken just after it occurred. The next stage is the level two (I.2) trigger system. If the L1 decision is not confirmed then the readout process at L2 is stopped and reset. The decision is taken before the next proton and anti-proton bunch corssing. L2 trigger selects only about $10 \%$ of all events saved by L1. At the last stage, level 3 trigger system, only about $2 \%$ to $5 \%$ of the events accepted after 1,2 are selected. Here the filtering of events is performed by software off-line, which indicates that it is much after the event has taken place and after it has been fully reconstructed. It has access to the information from all the sub-systems of the D $\varnothing$ detector.

\subsection{On-line trigger selection}

This section deals with the event selection procedure applied at the L1. Preliminary information about the final-state of a physics process is first obtained via this trigger system. The importance and classification of the event is based on a pre-defined set of conditions, called L1 filters. If the event is rejected by the L1 filters, then it is lost. However, if the event meets the filter requirements it is passed to the $I_{2} 2$ stage. The success of a filter for an event, (also called trigger firing) indicates the presence of one or more final-state objects sought in the event. Since various subdetectors measure response independently, one or more conditions can be used for cvent sclection, using boolcan AND and OR logic syntax.

The set of conditions that need to be net at the L1 stage are illustrated in 
Table 6.1 and the sub-section that follows describes the L1 objects which are used in the analysis.

\subsubsection{L1 EM objects, jet objects and muons}

Physics study involves analysis of off-line objects like the electromagnetic, muon and jet objects. These objects are reconstructed by the reconstruction farms ${ }^{2}$. In order to trigger on interesting events, one needs to use on-line information, for example from the L1 trigger.

\begin{tabular}{cc}
\hline $\begin{array}{c}\text { analysis } \\
\text { channel }\end{array}$ & $\begin{array}{c}\text { L1 trigger } \\
\text { name }\end{array}$ \\
\hline$e \mu$ & $\begin{array}{c}\text { mulotxatxx_CEM }(1,3) \\
\text { mulpt3wlxx_CEM }(1,3)\end{array}$ \\
\hline di-electron & CEM $(1,11)$ \\
& CEM $(2,6)$ \\
& CEM $(2,3)$ CEM $(1,9)$ \\
\hline di-muon & mu2ptxat.xx \\
\hline
\end{tabular}

Table 6.1: Triggers applied at the L1 stages for selecting di-lepton events.

The transverse encrgy $E_{T}$ of the trigger towers (TTs) is used to study the response of the L1 trigger tower readout. However, if the complete TT information is used, then the TT describes a L1 jet object. The EM TT which is fired constitutes the L1 clectromagnetic object, while the jet TT which is fired is the L1 jet object. At L1 one can determine the number of TTs (EM as well as jet TT) which satisfy the $E_{T}$ threshold levels.

The CFM triggers are termed $\operatorname{CFM}\left(N, E_{T}\right)$, where $N$ is the number of E.M objects fired by the trigger having the threshold $E_{T^{\prime}}$. A single EM trigger, $\operatorname{CEM}(1, x)$

\footnotetext{
${ }^{2}$ A host of stand alone processors constitute a farm.
} 


\begin{tabular}{lr}
\hline $\begin{array}{l}\text { analysis } \\
\text { channel }\end{array}$ & $\begin{array}{r}\text { L3 trigger } \\
\text { name }\end{array}$ \\
\hline$e \mu$ & MU_A_EM10 \\
& MATX_EM6_L12 \\
\hline di-cloctron & 2EM_HI \\
F_1_2I,20 & F_2_2L 20 \\
F_3_2I 20 \\
\hline di-muon
\end{tabular}

Table 6.2: Triggers applicd at the L3 stage for sclecting di-lepton events.

fires when there is at least one EM 'T' with $E_{T}^{\top}>x$. In an event that passed such a trigger we assume that the highest $E_{T}$ TT in the precision readout fired the trigger.

The scintillator detector as well as the drift tube's response dictate the presence of L1 muon objects. Favorable response from the muon detectors obtained alter the bunch crossing are attributed to cosmic muons. These objects are eventually rejected. Detcetor hits constitute the L1 muon objects.

Some I.1 trigger tower studies can be found in Appendix F and more details are available $[55]$.

\subsubsection{L2 EM objects, jet objects and muons}

Events that pass the L1 requirements are filtered at L2. For the em objects, simple cone algorithms are used to process the L1 TT responsc and form cluster(s) of em objects at L2. Jet clusters are also formed using the L1 jet objects. It can 
be determined at this stage if the EM or jet TT which fired in the event is isolated or not. The summation of the transverse energy in the clusters of TTs can now be defincd as well.

At 1,2 it is possible to determine the number of hits in the scintillator detectors as well as the drift tubes for a L2 muon object. If a L1 muon object has:

1. at least 1 wire hit in the A layer drilt-tube detectors,

2. at least 1 scintillator hit in the $\Lambda$ layer scintillator detectors;

3. at least 1 wire hits in the B,or $\mathrm{C}$ layer drift-tube detectors, or at least 1 scintillator hit in the $\mathrm{B}$, or $\mathrm{C}$ layer scintillator detectors,

then it is referred to as a 'loose quality' muon object. However, if a L1 muon object has:

1. at least 1 wire hit in the $\Lambda$ layer drift-tube detectors,

2. at least 1 scintillator hit in the A layer scintillator detectors.

3. at least 2 wire hits in the B,or C layer drift-tube detectors,

4. at least 1 scintillator hit in the $\mathrm{B}$, or $\mathrm{C}$ layer scintillator detectors,

then it is classificd as a 'modium quality' muon.

For the di-muon channel event selection at least one medium muon object is required. In the e $\mu$ channel there are no additional restrictions for muon objects or EM objects at L2. However, for the di-electron channel, it is required that there be two $\mathrm{L} 2 \mathrm{~cm}$ chusters over a $p_{T}$ threshold of $10 \mathrm{GeV}$. 


\subsubsection{L3 EM objects, jet objects and muons}

At L3, quality cuts can be applied on L2 EM objects to characterize them further. The following attributes of the $\mathrm{L} 3 \mathrm{em}$ object can be determined:

A. $f_{E M}$ : EM fraction, this is the ratio of the EM energy deposited in the EM layers of the calorimeter and the total energy of the cluster (which includes contributions from hadronic layers if any). The higher this ratio, the more likely it is that the cluster response is from an electromagnetic object.

B. $f_{i s o}$ : the isolation of an FM cluster is measured by comparing only the FM energy of the cluster within a cone of radius 0.2 to the total cluster encrgy within a radius of 0.4. Quantitatively, the em isolation fraction is defined as $f_{\text {iso }}=\frac{E_{\text {lotal }}(0.4)-E_{F, M}(0.2)}{E_{F M}(0.2)}$ C. shower width: The width of shower shape of the EM clusters in the three innermost EM layers can also be determined ${ }^{3}$.

L3 jet objects are also clusters ol energy in the calorimeter which are selected from I.2 jet objects. Compared to FM objects, jet objects are wider in the $(\eta, \phi)$ spread, algorithms are used off-line to reclassify and categorize these objects. IIowever, at L3 some characteristic information is available as well. The fraction of jet cncrgy in the coarse hadronic layers, compared to that in the fine hadronic layers can be determined.

L3 muon objects are similar to L2 muon objects. However, at L3 the muon

\footnotetext{
${ }^{3}$ The shower shape is re-established off-line as well. However at L3 there is an added advantage. Maximum energy is deposited in the third EM layer by EM objects when they shower in the material of the calorimeter. The finer granularity of the third layer is an advantage, and it provides good encrgy resolution for the em clusters. Due to the presence of iron toroid and the pre-shower the EM shower initiation oceurs before the EM objects hit the calorinneter itself and do not initiate in the first EM layer. It is possible that the maximal energy of the EM cluster may not be deposited in the third layer, but in the second layer. Therefore sampling of the cluster width in the first three layers of the calorimeter provide a useful discriminant at L3 as well.
} 
objects from L2 can be used in conjunction with track inlormation from the CFT and the SMT, confirming the presence of a muon track object. This is implemented in the L3 muon triggers for the di-muon channcl, where at least one muon track object is required.

\subsection{Off-line reconstruction}

Optimal use of all saved information is made to understand the response in the detector. The process in which information from all detector subsystems is incorporated to reveal the signatures of the physical particles produced in the event is called off-line reconstruction. It is also known as 'reco'. Software is used for all off-line reconstruction.

Over 50 thousand detector electronic channels carry information ofl-line for the reconstruction of the physics event. Information from only those events which pass the trigger requirements are saved to peripheral devices e.g. tapes. The main sequences are the reconstruction of:

- The track objects in the event.

- The primary vertex, using the track objects.

- The electron objects, muon objects and jet objects.

- The unbalanced transverse momentum using all reconstructed objects, clustered as well as unclustered cnergy in the calorimeter.

Well defined set of selection criteria are used, each of which has to be met for the reconstructed object to be considered valid. This cnsures a larger fraction of events from the selected sample having the characteristic event signature. 
The Section 6.5 describes the set of selection eriterion used, while ensuing subsections describe the reconstruction of various objects in the event.

\subsubsection{Reconstruction of a track object}

The tracking detectors record hits or clusters of hits from charged particles. Algorithms are used to find and fit the tracks in the event using the collection of clusters or hit information from one or more of the sub-detectors. 'The mathematical equation which indicates a possible particle trajectory in the event is called a track. Therefore the track object is a re-creation of a possible trajectory which the particle in an cvent may have followed.

Once the track objects are defined, the next step is to reconstruct the primary and the secondary vertexes. However, in this thesis the secondary vertexes are not used, and will therefore not be discussed.

\subsubsection{Reconstruction of the primary vertex}

While the incoming proton and anti-proton bunches are focused at the nominal interaction point, the actual point of collision may however be different. Algorithms which use track objects as inputs, are used to identify the possible position of the impact. Reconstructed tracks are used in conjunction with the beam spot information to determine this point. This reconstructed point is defined as the primary vertex.

Once tracks to be used in the event are selected, a clustering algorithm is used to identify tracks belonging to different interactions. The clustering algorithm bunches neighbouring tracks in a $2 \mathrm{~cm}$ segment along the $z$ axis. Within each cluster the 
tracks are fitted to a common vertex using a Kalman Filter[־6] algorithm. The best fit determines the position of the primary vertex, and all tracks in the same event are refitted with the requirement that they originate from the new vertex position.

More than one hard scatter may occur in the same bunch crossing. Therefore it is possible to reconstruct more than one primary vertex. Instrumental effects like tracking resolutions, or mis-identificd tracks can give rise to spurious primary vertexes. The selection of a primary vertex to be used in reconstruction is based on the track multiplicity or on the transverse momentum of the associated tracks. The optimal selection may depend on the physics process. For the $t \bar{t}$ events it was established that the sum of the logarithms of the transverse track momenta gives the best discriminator in finding the primary vertex[57].

The identification of the primary vertex is crucial for an accurate measurement of the transverse momentum of all objects in the events, e.g. the clectron objects, muon objects, or jet objects as well as the imbalance in transverse monentum.

\subsubsection{Reconstruction of muon objects}

Muon objects are reconstructed using information from the tracking detectors, as well as the muon detectors which are located outside the calorimeter. A L3 muon object in conjunction with a geometrically matched track object would correspond to an off-line muon track. An estimate of the muon momentum is obtained from the bending angle of the muon track in the toroidal magnetic ficld. Further details

will be discussed in the next section. A muon track object in the calorimeter cell (MTC) is reconstructed as well[58]. 


\subsubsection{Reconstruction of electron objects}

Ofl-line, cnergy inlormation from all calorimeter colls is available. Rc-clustering of energy depositions into simple cone objects of radius 0.2 units is done. The cluster energy can also be determined. The segmentation of the calorimeter provides measurements of the longitudinal shower shape as well as the transverse shower shape of energy depositions. In addition the Central Pre-Shower detector (CPS) detector provides energy measurement as well as the cluster shapes of these objects since the shower development is initiated in the CPS. The CFT and the Si detector provide precise matching with the CPS cluster position, and they provide means to measure the transverse momentum $\left(p_{T}\right)$ as well as the ratio $(E / p)$.

The L3 em objects which have an associated track object are said to be 'tight' clectron objects. The algorithm for obtaining the isolated clectron objects uses calorimeter clusters which are matched with the CPS information. These in turn are then matched with tracks. Isolated clusters and isolated tracks are only sclected for this analysis.

Off-line all qualitative information from L3 electron objects are either refined or preserved. 'These features, described carlier in Section 6.3.3, are:

- The EM fraction of energy in each cluster.

- 'The em isolation.

- Then, using the H-matrix technique one can compare observed shower shapes to expectations using the covariance matrix of energy deposits in different calorimeter layers. This leads to a composite variable for discriminating shower shapes of electron and photon objects and other hadrons ${ }^{4}$.

\footnotetext{
${ }^{4}$ To determine the electron/photon likeness of a shower, the electron response is generated using
} 
Table 6.3 summarizes the algorithms used lor defining an electromagnetic cluster (object). For this analysis, only the first algorithm is used.

\begin{tabular}{|c|c|c|c|c|}
\hline seed & isolation cut & associated track & energy info & angular info \\
\hline cluster & yes & yes & EMcluster & Track \\
SEM + cluster & ycs & yes & EMclustcr & Track \\
cluster & ycs & no & EMcluster & cal/ PS \\
SEM & ycs & ycs & Track & Track \\
SEM & no & yes & Track & Track \\
\hline
\end{tabular}

Table 6.3: $\Lambda$ list of electron ID definitions used in reconstruction algorithms.

\subsubsection{Reconstruction of jet objects}

The algorithm used for the off-line reconstruction of jet objects is the Improved Legacy Cone Algorithm[59]. The algorithm aims to reconstruct all clusters of calorimeter energy depositions as fixed radii cones in $(\eta, \phi, r)$ space.

Every calorimeter cluster is assigned to be a massless 4 -vector object, with the direction of the object corresponding to the trajectory and the energy of the object as its scalar component. All such 4-vector objects within a pre-determined cone size are combined, and various fixed radius conc configurations are obtained. An algorithm is used for clustering particles, partons or even energy depositions. For this analysis, the algorithm uses a fixed cone of radius 0.5 units. The algorithm is modeled such that each of these cones contain stable jets, i.e the jet axis and the 4-vector sum of all the calorimeter objects are as 'close' as possible.

the detector simulations. Then, for example for a sample of $N$ simulated electrons, one can define a covariance matrix. Then the $\chi^{2}$ which measures the consistency of a shower with a typical $\mathrm{em}$ shower can be defined. This value of the $\chi^{2}$ is used as a discriminating valuc. There are 8 observables used in constructing the $\chi^{2}$, they are: fractional shower energy in each of the 4 EM layers of the calorimeter, the shower widths along the two transverse directions, the logarithm of the total energy, and the longitudinal position of the event's primary vertex. 
During reconstruction it is possible to decipher jets which include delective or noise calorimeter cells, or TTs. Their contribution in cluster energy can be determined, and avoided as well.

\subsubsection{Corrections to off-line objects}

The reconstructed electrons, muons, and jets are calibrated. This involves a series of corrections which will be described in Chapter 7.

\subsubsection{Determination of the unbalanced transverse momentum}

$\Lambda$ fter full reconstruction of all objects in an event is achieved and after nocessary corrections are applied to those objects, the imbalance in the transverse momemtum is estimated. 'The response is attributed to the presence of undetected neutrinos in the final-state of the event

\subsection{Selection cuts}

Two main types of criteria are imposed for event selection. Data quality criterion are imposed to remove known corrupt runs and luminosity blocks. Sccondly, cvent selection cuts are imposed to enhance high signal-to-background ratio. This section deals with the latter issue. 


\subsubsection{Selection cuts for track objects used in reconstructing the primary vertex}

The characteristics of the tracks used for primary vertex reconstruction are:

- The $p_{T}$ of tracks to be $\geq 0.5 \mathrm{GeV}$.

- SMT hits $\geq 2$.

- DCA significance of track objects $\leq 3.0$.

\subsubsection{Selection cuts for the primary vertex identification}

The sclection critcria for the primary vertex are:

- The absolute value of the longitudinal spread of the PV from the center of the detector $\left(\left|z_{0}\right|\right)$ be $<60.0 \mathrm{~cm}$. This criterion ensures that the primary vertex is reconstructed within the tracking volume of the silicon detector.

- At least threc tracks are associated with the primary vertex.

Further details regarding the primary vertex selection criteria and its characteristics are available in $[60]$.

\subsubsection{Selection cuts for muon identification}

In addition to the medium muons described above, further cuts are applied on the muon objects. Tracks reconstructed using the muon detectors are extrapolated to the point of closest approach (PCA) to the beam, and moreover these parameters are compared with tracks from the tracking subsystems at the point of closest approach as well. $\Lambda$ global fit is performed with all ccntral tracks within 1 radian in azimuthal 
and polar angle of a muon track at PCA. The central track with the highest $\chi^{2}$ probability is considered as the muon candidate. The measurement of the muon track parameters is taken from the tracking detectors. 'This identifies a muon object whose origin is consistent with that of one coming from the primary vertex. In addition,

- The $(r, \phi)$ distance ol closest approach (dea) significance, defined as the ratio of dca to its error, is limited to $|d c a| / \sigma_{d c a}<3.0$.

- The distance along the beam direction from the muon to the primary vertex is also constrained to $|\Delta z(\mu, P V)|<1.0 \mathrm{~cm}$.

It is difficult to determine the radius of curvature of high $p_{T}$ muon objects for the stiff tracks. We avoid abnormally large $p_{T}$ muons from the signal samples which tend to be matched to poorly reconstructed tracks by restricting the fit to the matched track using $\chi_{\text {track }}^{2}<1.0$.

Background processes containing $b$ jet decays may give rise to high $p_{T}$ muon objects too; however, these muon objects are not well isolated from the jet objects in the event. An isolation variable devised on the ratio of the visible energy (halo) surrounding the muon and its $p_{T}$ is estimated. Specifically, it is required that:

- $\operatorname{Halo}(0.1,0.4) / p_{T, m u o n}<0.12$.

- $\operatorname{TrkConc}(0.5) / p_{T, m u o n}<0.12$.

- For high $p_{T}$ muon objects, $p_{T}>15.0 \mathrm{GeV}$ is used. 


\subsubsection{Selection cuts for electron identification}

After initial identification of an electron object, we can cnhance the quality of the object by further imposing quality cuts. The qualitative requirements, described previously in section 6.4 .4 , are:

- $\int_{E M}>0.9$.

- $f_{\text {iso }}<0.15$.

- $h m x 8 \chi^{2}<75.0$

- Electrons are required to pass the likclihood $(L)$ cut of: $L>0.85$. This cut has been revised ${ }^{5}$.

- The electron candidates are also required to have an associated track.

If an electron satisfies all the criteria mentioned and has a $p_{T}>15.0 \mathrm{GeV}$, then they arc sclected.

\subsubsection{Selection cuts for jet identification}

For sclecting jet objects in the events, the following cuts are applied to reconstructed events:

- $\Lambda$ cut on the fraction of cnergy deposited in the elcetromagnetic calorimeter $f_{E M}$ is applied $0.05<f_{E^{\prime} M}<0.95$.

- $p_{T}>20.0$.

${ }^{5}$ For the analysis done in spring 2004, electrons in the central calorimeter were selected with a. likclihood cut of $L>0.75$, and clectrons which are in the end calorimeter have a tighter likelihood cut of $L>0.80$. 


\subsection{Expected signal and background yields}

After application of selection cuts, the expected signal and background yields were established[52] from data as well as the simulated Monte Carlo generated events. Table 6.4 highlights the expected background and signal yields in the di-electron channel, for the data sample of $243.00 \mathrm{pb}^{-1}$ used, while Table 6.5 corresponds to the expected background and signal yiclds in the di-muon channel, lor the data sample

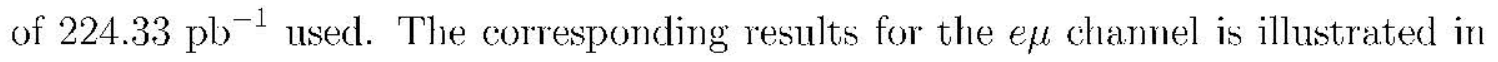
Table 6.6 which uses data sample of $228.29 \mathrm{pb}^{-1}$.

\begin{tabular}{c|c|c|c}
\hline $\begin{array}{c}\text { process } / \\
\text { category }\end{array}$ & $\begin{array}{c}\text { event } \\
\text { yield }\end{array}$ & $\begin{array}{c}\text { statistical } \\
\text { uncertainty }\end{array}$ & $\begin{array}{c}\text { systematic } \\
\text { uncertainty }\end{array}$ \\
\hline inclusive $Z / \gamma^{*}$ & 0.13 & \pm 0.03 & +0.03 \\
inclusive $W W$ & 0.14 & \pm 0.05 & ${ }_{-0.07}^{+0.07}$ \\
\hline instrumenlal lakcs & & & \\
missing $E_{T}$ lakes & 0.59 & \pm 0.09 & 0.00 \\
EM fake & 0.07 & \pm 0.03 & 0.00 \\
\hline total bkg & 0.93 & \pm 0.11 & -0.09 \\
\hline expected signal & 1.91 & \pm 0.05 & -0.28 \\
\hline \hline \# selected events & 5 & & \\
\hline \hline
\end{tabular}

Table 6.4: The expected signal and background yields and number of events for the di-electron channel[52]. The expected signal yield assumes a $7 \mathrm{pb} t \bar{t}$ production cross-scetion. 


\begin{tabular}{c|c|c|c}
\hline $\begin{array}{c}\text { category/ } \\
\text { process }\end{array}$ & $\begin{array}{c}\text { event } \\
\text { yield }\end{array}$ & $\begin{array}{c}\text { statistical } \\
\text { uncertainty }\end{array}$ & $\begin{array}{c}\text { systematic } \\
\text { uncertainty }\end{array}$ \\
\hline$Z / \gamma^{*}$ & 1.14 & \pm 0.13 & ${ }_{-0.51}^{+0.30}$ \\
$W W$ & 0.16 & \pm 0.02 & ${ }_{-0.06}^{+0.07}$ \\
\hline instrumental & & & \\
fake & 0.07 & \pm 0.03 & ${ }_{-0.02}^{+0.02}$ \\
\hline total bkg & 1.37 & \pm 0.13 & ${ }_{-0.63}^{+0.39}$ \\
\hline expected signal & 1.55 & \pm 0.06 & ${ }_{-0.24}^{+0.20}$ \\
\hline \hline \# selected events & 0 & & \\
\hline \hline
\end{tabular}

Table 6.5: The expected signal and background yields, and observed number of cvents for the di-muon channel[52]. The cxpected signal yicld assumes a $7 \mathrm{pb} t \bar{t}$ production cross-section.

\begin{tabular}{|c|c|c|c|}
\hline $\begin{array}{c}\text { category/ } \\
\text { process }\end{array}$ & $\begin{array}{l}\text { cvent } \\
\text { yicld }\end{array}$ & $\begin{array}{c}\text { statistical } \\
\text { unccrtainty }\end{array}$ & $\begin{array}{l}\text { systematic } \\
\text { uncertainty }\end{array}$ \\
\hline$Z / \gamma^{*}$ & 0.38 & \pm 0.06 & $\begin{array}{l}+0.08 \\
-0.08\end{array}$ \\
\hline$W W$ & 0.36 & \pm 0.00 & $\begin{array}{l}+0.18 \\
-0.15\end{array}$ \\
\hline$\gamma-$ processes & 0.02 & \pm 0.02 & $\begin{array}{l}+0.01 \\
{ }_{-0.02}\end{array}$ \\
\hline $\begin{array}{c}\text { instrumenlal } \\
\text { Jake }\end{array}$ & 0.20 & \pm 0.02 & \pm 0.07 \\
\hline total bkg & 0.96 & \pm 0.07 & $\begin{array}{l}+0.21 \\
-0.18\end{array}$ \\
\hline cxpected signal & 5.22 & \pm 0.11 & $\begin{array}{l}+0.53 \\
-0.42\end{array}$ \\
\hline \# selected events & 8 & & \\
\hline
\end{tabular}

Table 6.6: Fxpected background yields, expected signal yield and observed number of events for the $e \mu$ channel[52]. The expected signal yield assumes a $7 \mathrm{pb} t \bar{t}$ production cross-section. 


\subsection{Selected data sample}

After the application of all selection cuts, and removal of all runs with poor quality of the detector response, 8 events were selected in the $e \mu$ channel, 5 were selected in the di-electron channel and none were selected in the di-muon channel. The run numbers and event numbers for the selected di-electron events are given in Table 6.7, and the sclected $e \mu$ cvents in Table 6.8 .

\begin{tabular}{|r|r|}
\hline run number & event number \\
\hline 177681 & 13869716 \\
180326 & 14448436 \\
166779 & 121971122 \\
178152 & 26229014 \\
178177 & 13511001 \\
\hline
\end{tabular}

Table 6.7: Run numbers and event numbers for the selected events in the di-clectron channel.

\begin{tabular}{|r|r|}
\hline run number & event number \\
\hline 178733 & 8735139 \\
179111 & 11709332 \\
179195 & 2638170 \\
178159 & 37315438 \\
177826 & 15259654 \\
179331 & 19617819 \\
174901 & 8710859 \\
168733 & 1997007 \\
\hline
\end{tabular}

Table 6.8: Run numbers and event numbers for the selected events in the e $\mu$ channel. 


\section{Chapter 7}

\section{Detector Calibration and}

\section{Resolution}

This chapter addresses the calibration of the 4-vectors of the final-state particles. While the precision of the relevent measurement is dependent on the inherent resolution of detector sub-systems, its accuracy is achieved via energy calibration using well-measured, easy to resolve, and well established resonances ${ }^{1}$. For selecting candidate events from collider data and measure the mass of the top quark using the selected events, it is essential to measure the 4-vectors of the final-state objects.

In proton and anti-proton collisions, it is difficult to account for the momentum of all final-state particles which fly along the proton anti-proton beam axis. These hard-to-detect remnants can possibly carry a substantial fraction of the total energy along the beam-pipe. Moreover, the detector is absent for the high $\eta$ range $(|\eta| \sim 4)$. Therefore, it is not possible to estimate the 4 -vectors of all particles. Since there is no initial momentum along the transverse dircetion of the beams, the vector

\footnotetext{
${ }^{1}$ For example, we will not yet try to use the now resonance state, which the Sclex experiment at Fermilab claims to have discovered[61].
} 


\begin{tabular}{llr}
\hline $\begin{array}{l}\text { physics } \\
\text { study }\end{array}$ & $\begin{array}{l}\text { analysis } \\
\text { channel }\end{array}$ & $\begin{array}{r}\text { calibrated detector } \\
\text { sub-system }\end{array}$ \\
\hline high $p_{T}$ & $Z \rightarrow e^{+} e^{-}$ & em-calorimeter / tracking \\
high $p_{T}$ & $\pi^{0} \rightarrow \gamma \gamma$ & em-calorimeter \\
high $p_{T}$ & $\gamma+j e t$ event & hadron-calorimeter \\
high $p_{T}$ & $Z+j e l \rightarrow e^{+} e^{-}+j e l$ & hadron-calorimcter \\
high $p_{T}$ & $Z \rightarrow \mu^{+} \mu^{-}$ & muon systcm / tracking \\
\hline low $p_{T}$ & $J / \psi \rightarrow \mu^{+} \mu^{-}$ & tracking \\
low $p_{T}$ & $J / \psi \rightarrow e^{+} e^{-}$ & tracking \\
\hline
\end{tabular}

Table 7.1: Physics processes used in the energy-momentum calibration. The value of the resonance mass is obtained from the Particle Data Group[64]. High $p_{T}$ physics involves objects which are $\sim 10.0 \mathrm{GeV}$ or higher.

sum of the transverse momentum of all final state products can be constrained to the null value. This is essentially crucial for estimation of the missing transverse encrgy in an event and in calibration of the jet energy. The trackiing system is used to establish the transverse momentum of charged particles. This motivates the calibration of the momentum of the final-state muons and clectrons particles in the transverse plane. For the case of electrons and jets, the shower development in the calorimeter makes it impossible for momentum estimation of the plethora of gencrated daughter particles. Estimation of the shower cnergy can be achicved via the response (deposited charge) of the daughter particles in the active layer of the calorimeter cells.

Table 7.1 shows some of the physics processes used in the energy-momentum calibration of various detector sub-systems. Electron pairs produced from known resonances, e.g. the $Z$-boson, are used to calibrate the encrgy scale of the cmcalorimeter and to determine the position and momentum resolution. A procedure similar in style is adopted, using the muon pairs from those resonances, to cstablish the transverse momentum scale for the muon system. The transverse momentum 
scale is then adapted for obtaining the momentum scale as well. The position resolution of the tracking system and the issues of jet energy calibration and the jet encrgy resolution are discussed.

\subsection{Calibration of the electron energy scale}

The absolute energy scale of the calorimeter modules was established[62] before Run I commenced. A controlled beam of electrons was used to calibrate the electromagnetic calorimeter's response. After obtaining a preliminary calibration, the detector is re-calibrated $i n$-situ using collider data. This avoids potential effects due electronic noise from the readout system (which is different from that of Run I). The electronic coupling (hardware coupling) to the detector may result in an electronic response which diflers from the original response.

This thesis deals with the measurement of high $p_{T}$ (i.e. $p_{T} \sim 10 \mathrm{GeV}$ or higher) electrons. For the electromagnetic calorimeter calibration at high transverse energy i.e. $E_{T} \sim 15.0 \mathrm{GeV}$ or higher, electron pairs from the $Z$ resonance decays are used to reconstruct the on-shell $Z$ resonance. The measured 4 -vector $\left(E_{i}^{r e c o}\right)$ of the decay products is then corrected using

$$
E_{i}^{\prime}=\alpha E_{i}^{r \epsilon c o}+\beta,
$$

in addition to a kinematic constraint on the invariant $Z$ mass as shown by J. Zhu[63]. This helps scale the reconstructed $Z$ pole mass to the more accurate value obtained from the Particle Data Group[64] as well as from the LEPEWWG[65]. In Equation $7.1 E_{i}^{\text {reco }}$ is the reconstructed energy of the $i^{\text {th }}$ electron, while $E_{i}^{\prime}$ is obtained after correcting that by the factor $\alpha$ and an offset $\beta$ such that the central value of the 
$Z$ resonance coincides with the value from that of the Particle Data Group ${ }^{2}$. These corrections are dependent on $\eta$, since response of the calorimeter in the central, forward or the inter cryostat region differ. However, the corrections are applicable to all high $p_{T}$ electrons irrespective of the underlying physics process. The width of the $Z$ resonance gives a measure of the mass resolution that can be obtained from the calorimeter.

Monte Carlo events are modeled to mimic the kinematic distributions from data. The value of the resonance mass of the $W$ boson and the $Z$ boson is from the Particle 1)ata Group, resolutions of the invariant mass distribution may differ due to our inability to simulate the accurate model of the detector. Therefore, a correction, known as the oversmearing correction, is applied to tune the electron energy response to match the resolution obtained from data events. The scalar value of the smeared 1-momenta, $E_{\text {smear }}$ is then represented as:

$$
E_{s m e a r}=E^{\prime}+x \sigma_{E},
$$

where $x$ is a random number obtained from a unit Gaussian distribution (RMS of unit value with the mean of zero), and $\sigma_{E}$ is the electron oversmearing resolution [66]. Once the value of the over-smeared energy is obtained, then the 4 -vectors are obtaincd using the original angular projections of the electron.

The central and the end-cap clectromagnetic calorimetcrs are structurally and functionally independent, the scaling and smearing corrections for electrons in these regions are obtained separately. Plots of the $Z$ resonance from data and simulated events are shown in Figure 7.1. The high $p_{T}$ di-electron invariant mass is

\footnotetext{
${ }^{2}$ This measurement is dominated by the results from the IFP experiments.
} 


\begin{tabular}{|l|l|l|}
\hline $\begin{array}{l}\text { detector } \\
\text { region }\end{array}$ & $\begin{array}{l}\text { scale } \\
\text { parameter }\end{array}$ & $\begin{array}{l}\text { oversmearing } \\
\text { parameter }\end{array}$ \\
\hline central (CC) - within fiducial & $1.003 \pm 0.001$ & $0.045 \pm 0.004$ \\
central (CC) - not in fiducial & $0.950 \pm 0.011$ & $0.115 \pm 0.009$ \\
End-cap Region (EC) & $0.996 \pm 0.005$ & $0.034 \pm 0.009$ \\
\hline
\end{tabular}

Table 7.2: The scale parameters and oversmearing parameters [66] applied to electron objects in the simulated events.

reconstructed from data events, and the distribution obtained is numerically fit to Gaussian function. The RMS of the best fit is used as a measure of the energy resolution. The details regarding the evaluation of the scale and smearing corrections are described by S. Jain in [66]. These oversmearing parameters and the scale factors obtained from S. Jain[66] are shown in Table 7.2. 

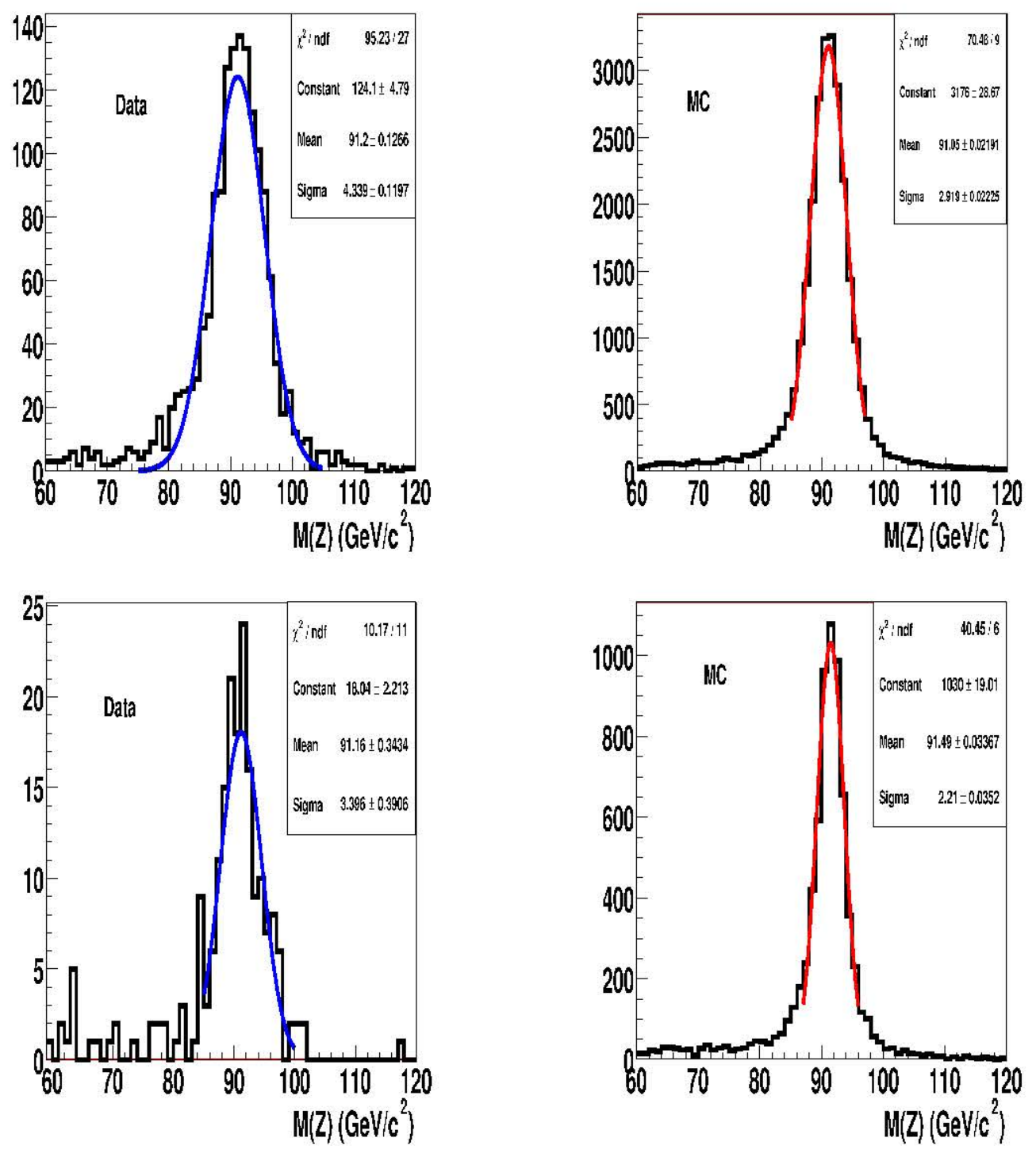

Figure 7.1: Comparative study of the reconstruction of the $Z$ resonance from Tevatron data and simulated $Z \rightarrow e^{+} e^{-}$cvents. The pair of plots on the top correspond to the case when both electrons used in the mass reconstruction are in the $\mathrm{CC}$ as well as the fiducial region of the detector. The bottom plots represent the case which have both electrons in the $\mathrm{EC}$ region. The region which is dominant in signal events is numerically fit using the Gaussian function, and the RMS of the fit obtained is used as a measure of the energy resolution. The plots are obtained from S. Jain[66]. 


\subsection{Calibration of the muon momentum scale}

A procedure similar in style is adopted for muons for reconstructing the $Z$ resonance from $Z \rightarrow \mu^{+} \mu^{-}$events. Muons are calibrated such that the mean of the resonance distribution corresponds to the value of the $Z$ pole obtained from the Particle Data Group[64]. The RMS of the distribution gives a measure of the mass resolution which can be obtained from the tracking system and the muon system. The scale and oversmearing corrections are applied to the MC muons so as to calibrate the muon momentum scale, which gives a realistic representation of the mass resolution obtained using the tracker and the muon system in conjunction.

Figures 7.2 and 7.3 illustrate the reconstructed $Z$ resonance from a pair of muons in data cvents as well as simulated cvents. 

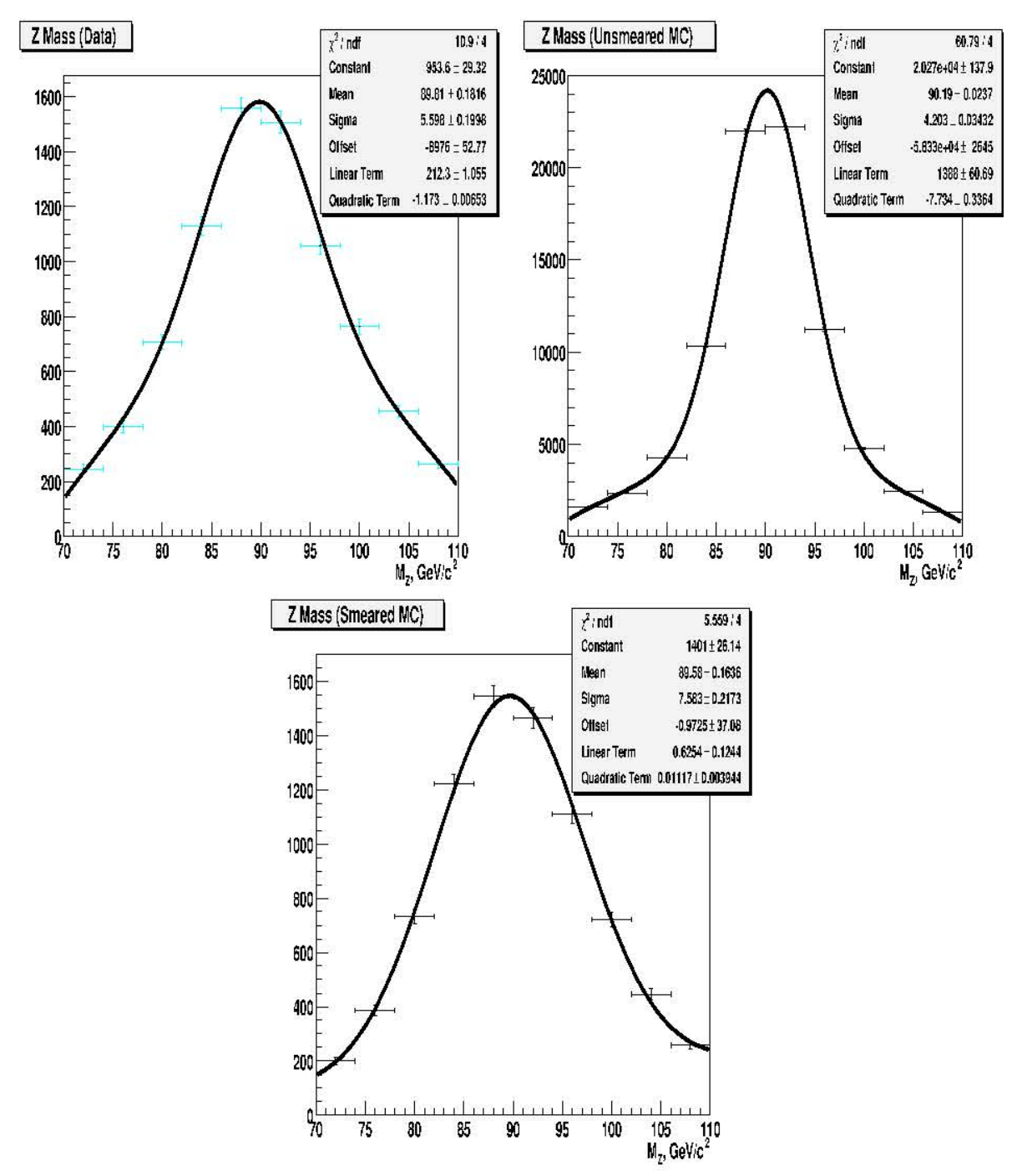

Figure 7.2: The $Z$ boson reconstruction from di-muon events detected in the central muon system. The left plot is from the Tevatron data, and the right plot is from MC events. The plot at the bottom is from MC events but with the scale and over smearing corrections applied to the muons. The horizontal crror bar represents the histogram bin width. All plots are obtained from D. Shpakov[67]. 

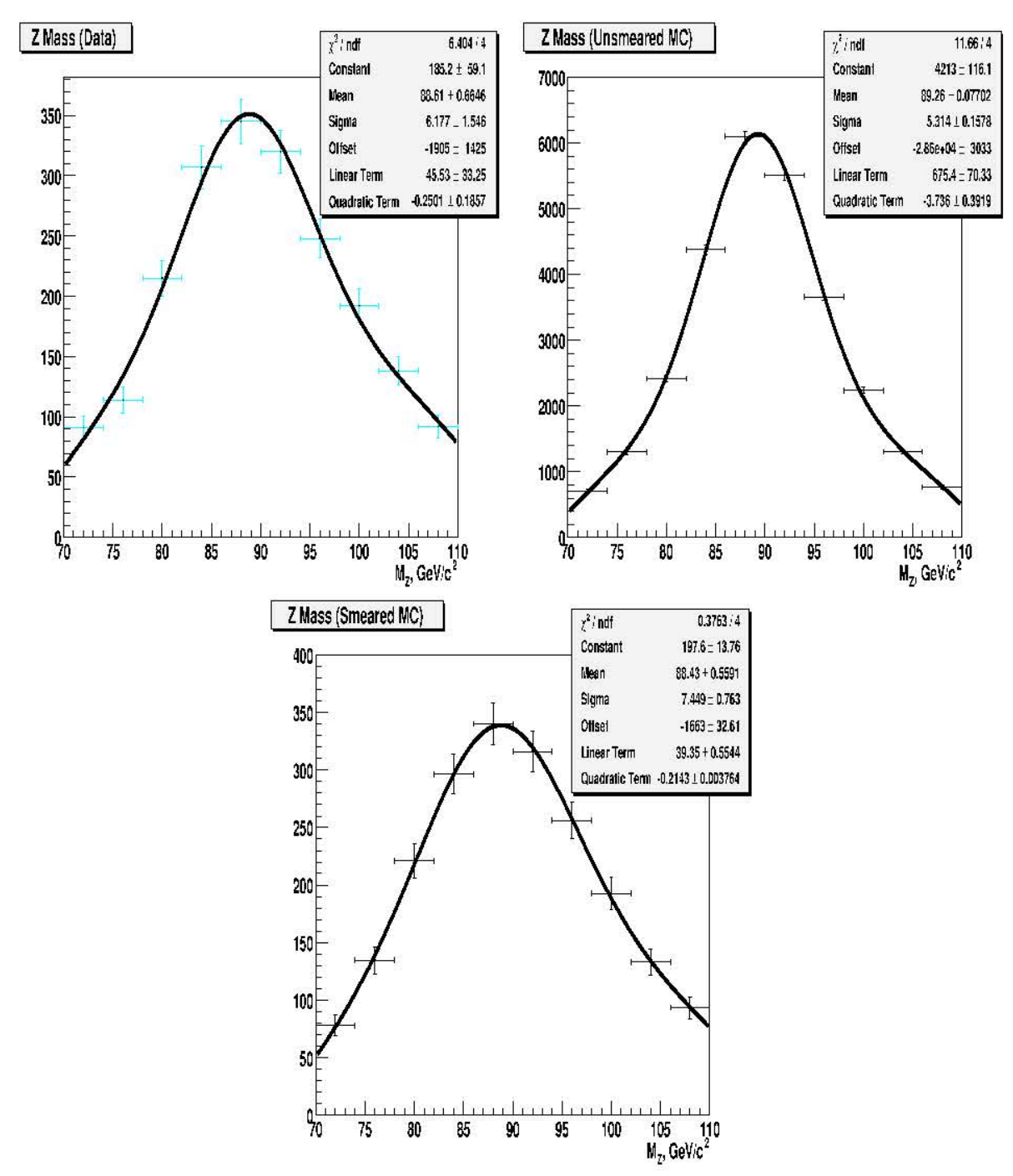

Figure 7.3: $Z$ reconstruction from di-muon pairs detected in the forward muon system. The upper left plot is from the Tevatron data, while the upper right one is from MC events. The bottom plot is from $\mathrm{MC}$ events but with the scale and over smearing corrections applied to the muons. The horizontal crror bar represents the histogram bin width. All plots are obtained from D. Shpakov[67]. 


\subsection{Calibration of the jet energy scale}

Figure 7.4 describes a schematic of the evolution of partons to energy depositions in the calorimeter. A cone algorithm is used to envelope clusters of energy deposition, to represent a consolidated object also known as the jet. Unlike the calibration of final-state electrons and muons, the energy of the final-state partons is non-trivial to calibrate. The jet encrgy calibration is typically done in two steps. First the response of the jet objects is calibrated to the detector level. Then the particle response is obtaincd. Finally, the response is calibrated in terms of the final-state partons.

This section describes the energy calibration of jets to yield an average response as if a collection of stable particles were the final-state objects. In the next section, the mapping of the response at the particle level (production stage) to the parton level (production stage) at hard-scatter is discussed.

The measured energy of the jet $\left(E_{d c t}\right)$ contained within a cone of radius $R$ is

correlated to the energy of the particles ( $\left.E_{\text {particles }}\right)$ that initiated the jet formation. The latter is a function of the jet's cone of radius $R$, pseudo rapidity with respect to the origin of the detector $\eta_{\text {det }}$, and the instantaneous luminosity $\mathcal{L}$, and is described in a $\mathrm{D} \emptyset$ collaboration Note [69].

$$
E_{\text {particles }}=\frac{E_{d e t}-\mathcal{O}\left(R, \eta_{d e l}, \mathcal{L}\right)}{\mathcal{R}\left(E_{d e t}, R, \eta_{\text {del }}\right) \times S\left(E_{\text {det }}, R, \eta_{\text {del }}\right)},
$$

where the factor $\mathcal{O}\left(R, \eta_{\text {det }}, \mathcal{L}\right)$ corrects for the energy deposited in the jet cone and does not, originate from the final-state particles. The lactor $\mathcal{R}\left(E_{d e t}, R, \eta_{d e t}\right)$ accounts for the non-linear response of the calorimeter material. The factor $S\left(E_{\text {det }}, R, \eta_{\text {det }}\right)$ accounts for the out-of-cone effects during the jet shower development. The following 


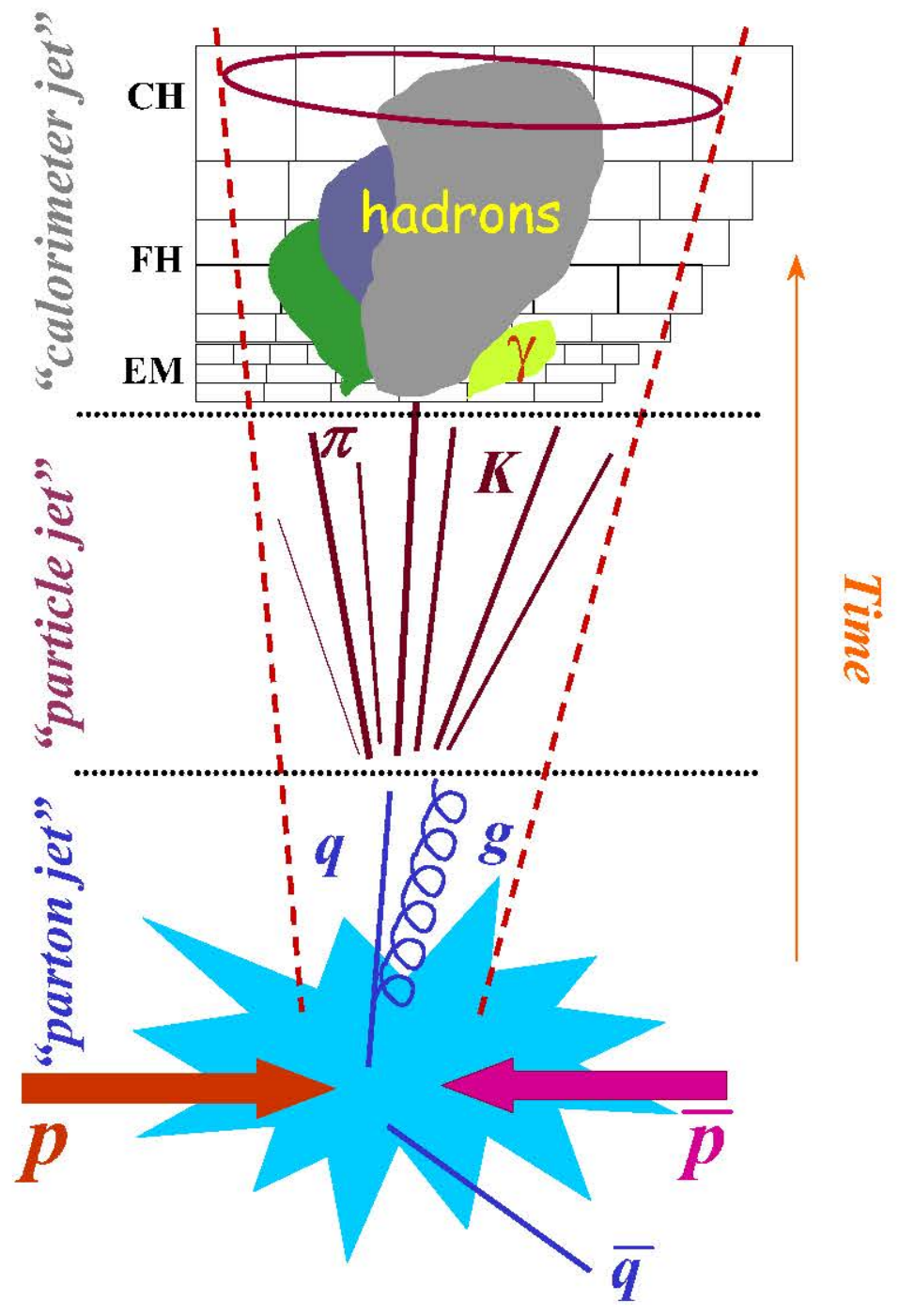

Figure 7.4: $\Lambda$ schematic representing the cvolution of partons to particles, then to energy cluster(s) in the calorimeter, and eventually to the jet enveloped by a hypothetical cone. 
sub-sections describe these lactors.

\subsubsection{Offset correction}

Jets manifest as clusters of encrgy deposition(s) in the calorimetcr. The deposition may occur in response to final-state particles and also may also occur due to:

- Uranium noise.

- Minimum bias interactions from beam crossings.

- Pile-up from previous beam crossings.

This correction factor is derived from a sample of events from proton and anti-proton collision having only the L0 (level zero) trigger confirmation ${ }^{3}$. Therefore, such cvents correspond to detected collisions which are not biased by any of the I, $1, \mathrm{~L} 2 \mathrm{2}$ or I,3 triggers.

The experimental procedure for such data acquisition is called a minimum bias run. The result yields a response called 'offset'. An average offset response is omitted from the response acquired during physics collisions. Figure 7.5 represents the transverse energy density per unit $\eta \times \phi$ as the function of the detector $\eta_{\text {det }}$.

\subsubsection{Response correction}

The response of the calorimeter does not scale linearly with increasing energy depositions. The correction is determined from the imbalance in the transverse cnergy in events having only two objects, one of which is an cm object. Since the cm cnergy scale is more preciscly determined, it is common practice that the response is

\footnotetext{
${ }^{3}$ This corresponds to the event confirmation obtained via the Luminosity monitors.
} 


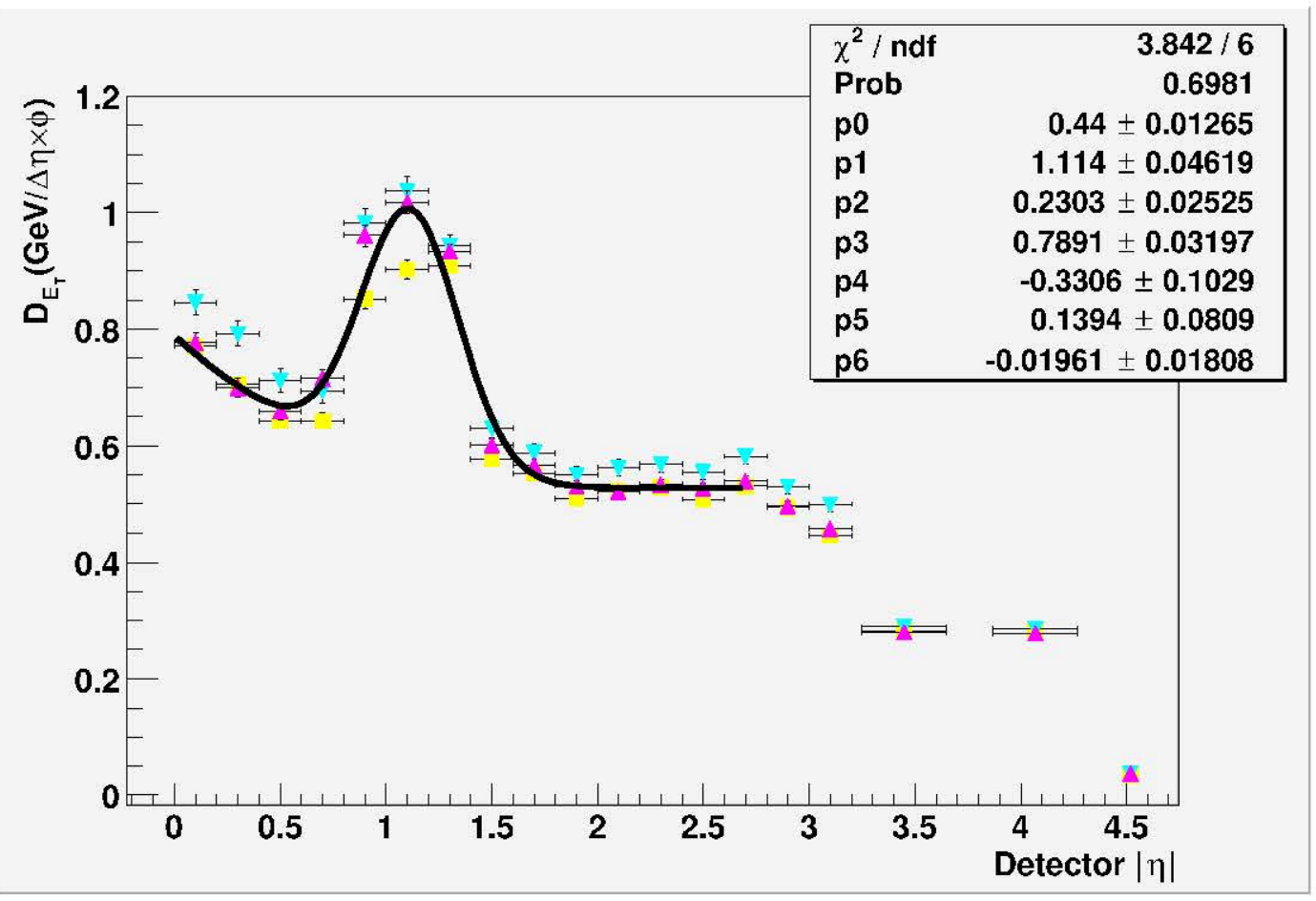

Figure 7.5: $F_{T}$ density per unit $\eta \times \phi$ as a function of detector $|\eta|$ in minimum bias data measured from a low (yellow squares), medium (pink upward triangles) and high (blue downward triangles) luminosity sample. The line represents a fit to the medium luminosity data. The horizontal crror bar represents the bin width. This plot is obtained from N. Parua[69].

determined from a hadronic jet object recoiling against a photon object. Therefore, after the electromagnetic scale has been determined, this response is calibrated. In this analysis jet algorithms with cone radius of 0.5 are used. Figure 7.6 shows the jet response lor 0.7 jet cone algorithms[69].

\subsubsection{Showering correction}

Reactions in proton anti-proton inelastic scattering can be described through interactions of initial-state partons that produce final-state partons. The final-state partons undergo hadronization and fragmentation. Hadrons from these partons 


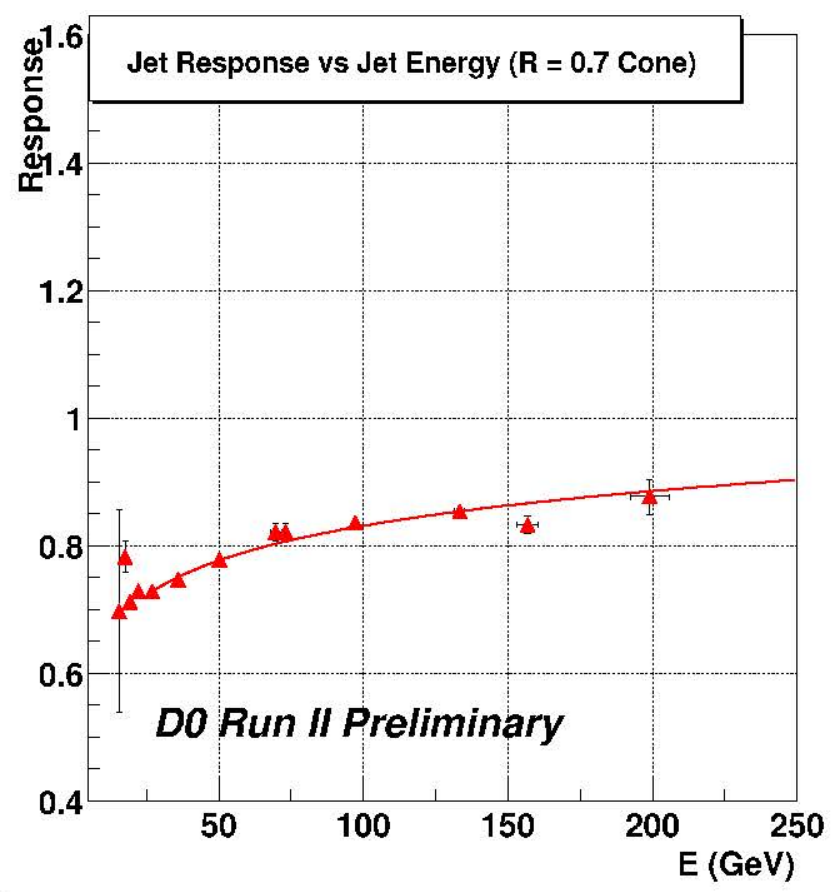

Figure 7.6: Jet cnergy response for a 0.7 cone algorithms. This plot is obtained from P. Perca[69].

interact with the calorimeter material yielding a shower (cascade) of particles. The character of individual hadron showers is independent of whether they originate from a gluon or a quark. However, quark and gluon jets differ in their fragmentation, and on average, quarks are known to produce narrower lateral profiles than gluons. Irrespective of the nature of the original parton initiating the shower, cone algorithms of fixed size are used to estimate the energy deposition in the calorimeter. Particles from within any such hypothetical cone can scatter and deposit energy outside the cone, while those from neighbouring un-clustered energy deposition, may leak in to the conc ${ }^{1}$. The showering correction accounts for these ellects on an average[70].

\footnotetext{
${ }^{4}$ In the case of events with three of more jets, cnergy may leak in from from particles of ncighbouring jots.
} 

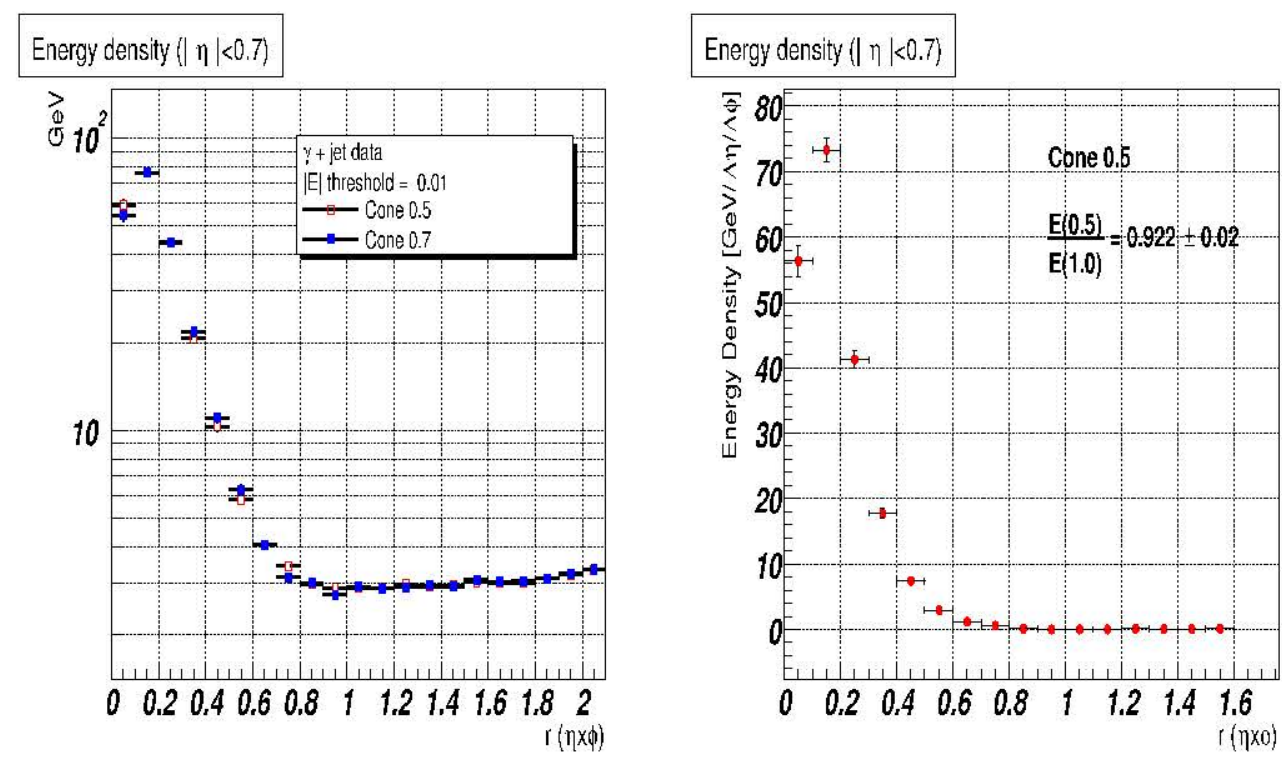

Figure 7.7: (Left) Energy density profiles from $\gamma+$ jet events in the central calorimeter as a function of the receding distance from the jet axis. (Right) The average encrgy density profile for jet objects for the central calorimeter after bascline subtraction. The horizontal crror bar represent the bin width of the histogram.

Independent corrections are obtained for the three calorimeter regions shown in Table 7.3. The corrections obtained for the data set used are derived from values of the jet energy contained within the fixed cone jet algorithm shown in Table 7.4. If the fixed cone algorithm were sufficient to describe the jet objects, then the fraction $F$ would always correspond to unity, and no correction would be needed. Figure 7.7(left) represents the encrgy density profile for the central calorimeter as a function of the receeding distance from the jet axis. The energy density within a cone radius of 0.5 can be estimated after bascline energy subtraction. T'This is shown in the Figure 7.7(right). A similar set of corrections is obtained for simulated MC events. Table 7.4 and 7.5 shows the average fraction of jet energy contained in fixed cone algorithms for data and simulated events respectively. 


\begin{tabular}{|c|c|}
\hline detector region & detector $\eta$ ranges \\
\hline central & $0.0 \leq|\eta|<0.7$ \\
inter cryostat & $0.7 \leq|\eta|<1.8$ \\
end cap & $1.8 \leq|\eta|<2.5$ \\
\hline
\end{tabular}

Table 7.3: Ranges of detector pseudorapidity used to obtain the jet energy calibration and associated corrections, e.g. the showering corrections. Identical detector pseudorapidity range is used to obtain other independent corrections with regards to reconstructed jets.

\begin{tabular}{|l|l|l|l|}
\hline detector $\eta$ & $J_{\lrcorner}$ & $\mathbf{F}_{\text {jet=0.5 }}=\mathbf{E}_{\text {jet=0.5 }} / \mathbf{E}_{\text {jet=JL }}$ & $\mathbf{F}_{\text {jet=0.7 }}=\mathbf{E}_{\text {jet=0.7 }} / \mathbf{E}_{\text {jet= }=\text { JL }}$ \\
\hline$|\eta|<0.7$ & 1.0 & $0.92 \pm 0.02$ & $0.99 \pm 0.02$ \\
$0.7<|\eta|<1.8$ & 1.2 & $0.89 \pm 0.02$ & $0.96 \pm 0.02$ \\
$1.8<|\eta|<2.5$ & 1.5 & $0.85 \pm 0.03$ & $0.94 \pm 0.03$ \\
\hline
\end{tabular}

Table 7.4: The average fraction of the jet energy contained in the fixed cone algorithms as a function of detector $\eta$ (from Tevatron data).

The calibrated jet energy is determined using the offset corrections, the response function, and the showcring corrcction using Equation 7.3.

\subsection{Evaluation of the missing transverse momentum}

There is no momentum component of the proton and anti-proton beam along the transverse direction. Due to conservation of momentum, after a proton antiproton collision we constrain the kinematics of each event to have a null transverse momentum. The vector sum of the imbalance in transverse momentum is denoted as the missing transverse momentum $\overrightarrow{p_{T}}$.

After the encrgy of the reconstructed jets and electrons, and the nomentum of the muons in the event are obtained, we then estimate the unbalanced momentum 


\begin{tabular}{|l|l|l|l|}
\hline detector $\eta$ & $J L$ & $\mathbf{F}_{\text {jct=0.5 }}=\mathbf{E}_{\text {jct=0.5 }} / \mathbf{E}_{\text {jct=.JL }}$ & $\mathbf{F}_{\text {jct=0.7 }}=\mathbf{E}_{\text {jct=0.7 }} / \mathbf{E}_{\text {jct=.JL }}$ \\
\hline$|\eta|<0.7$ & 1.0 & $0.94 \pm 0.02$ & $0.99 \pm 0.02$ \\
$0.7<|\eta|<1.8$ & 1.2 & $0.88 \pm 0.03$ & $0.97 \pm 0.03$ \\
$1.8<|\eta|<2.5$ & 1.5 & $0.66 \pm 0.10$ & $0.88 \pm 0.10$ \\
\hline
\end{tabular}

Table 7.5: The average fraction of energy contained in fixed cone jets as a function of detector $\eta$ (from MC events).

in the transverse plane. At this stage all smearing and scale corrections for the jets, electrons and muons have been applied. There may be energy depositons in the calorimeter that may lail to qualify as electrons or jets. Those depositions are categorized as un-clustered energy.

The transverse missing cnergy moasured using the calorimeter $\left(\mathbb{H}_{T_{c a l}}\right)$ is therelore estimated as:

$$
-\overrightarrow{\mathbb{Z}_{T}} \overrightarrow{\text { cal }}_{\text {all elcctrons }} \vec{E}_{T}^{\text {electron }}+\sum_{\text {all jcts }} \vec{E}_{T}^{\text {jet }}+\vec{E}_{T}^{\text {unclustered }}
$$

independently along the $x$ and $y$ axcs. After the calorimeter encrgy clusters have been used to extract the momentum, they are combined with the muon momenta to yield the imbalance in the event's transverse momenta as:

$$
-\overrightarrow{p_{T}}=\sum_{\text {all electrons }} \vec{p}_{T}^{\text {electron }}+\sum_{\text {all jels }} \vec{p}_{T}^{\text {jet }}+\vec{F}_{T}^{\text {unclustered }}+\sum_{\text {all muons }} \vec{p}_{T}^{\text {muons }} .
$$

\subsection{Correcting the jet 4-vector to represent the parton 4-vector}

Reactions in proton-antiproton inclastic scattcring can be described through interactions of initial-state partons producing final-state partons. The final-state partons 
undergo hadronization and fragmentation, and olten hard-gluon radiation. As cxplained before, the final-state partons manifest themselves as jets of particles, whose response can be measured with a detector. Hence, the 1-vector of any final-state parton is not identical to the 4-vector of the objects originating from those partons. A correction is therefore required to extract the 4-vector of the original hard parton from a jet. This correction, when applied to the jet, adjusts the 1-vector of the jet on average to that of the original parton.

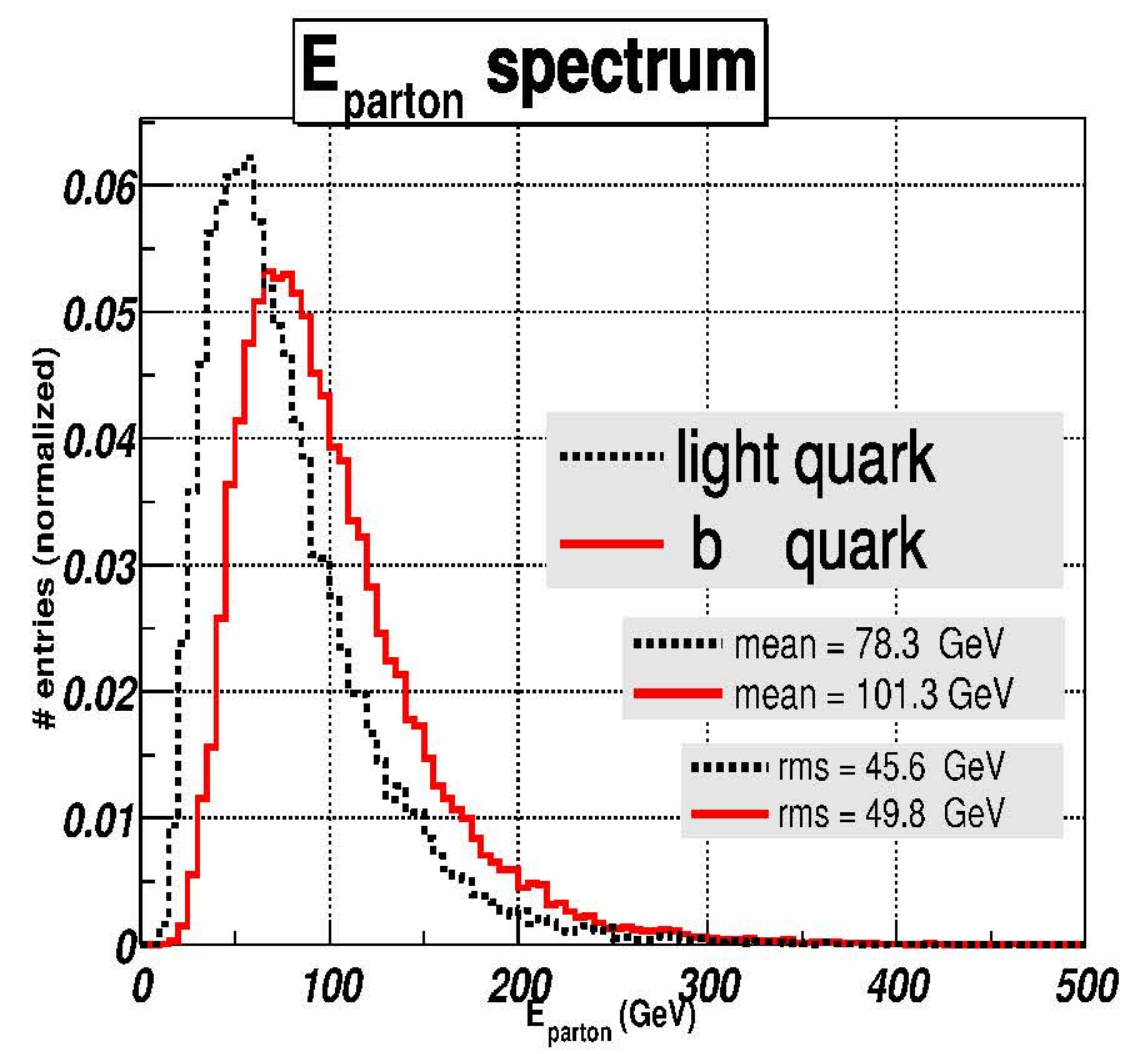

Figure 7.8: The energy spectrum of partons from light quarks and the $b$ quark. The number of entries in the two histograms are normalized to unity.

The energy spectrum of jet objects from simulated events $(t \bar{t} \rightarrow \mu+$ multi-jets process) originating from heavy-quark hadronization differs from those originating 
via light-quark hadronization ${ }^{5}$. Figure 7.8 shows the encrgy spectra in these two cases. The corrections are therefore derived separately for jets originating from fragmentation of light quarks $(u, d, s, c)$ and heavy quarks $(b)$ as a function of cnergy, and in three pseudorapidity bins of the IDØ detector, as shown in Table 7.3. For this study, simulated $t \bar{t}$ events are used in which one of the $W$ bosons produced in the hard scatter is forced to decay hadronically while the sccond $W$ is foreed to decay into $\mu$ and $\nu_{\mu}$.
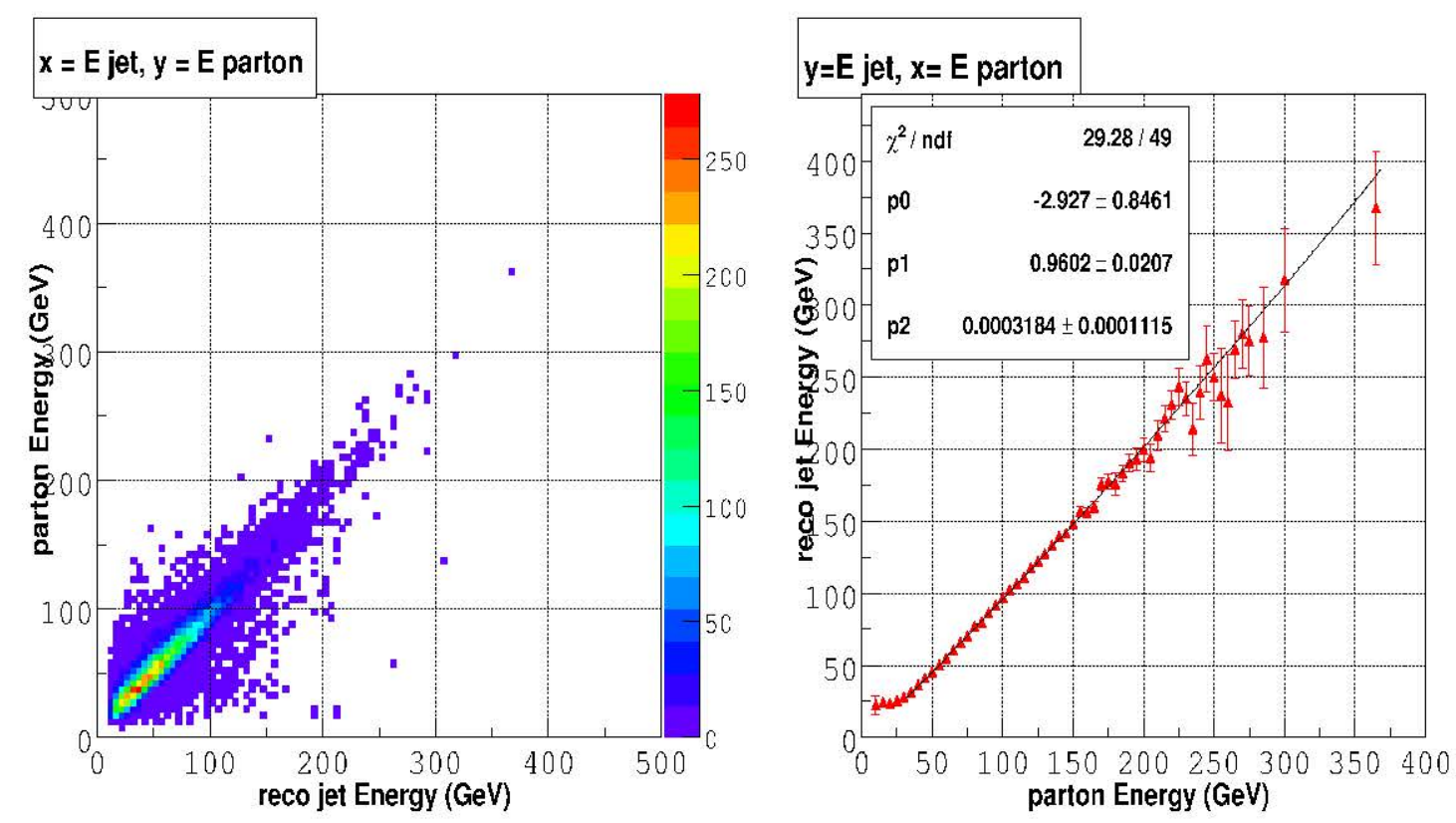

Figure 7.9: (Left) The encrgy of light-quark partons versus the energy of their bestmatched reconstructed jets. (Right) The average profile of the scatter plot at left.

Fitting the profiles of $E_{r \epsilon c o}$ versus $E_{\text {parton }}$ with $2^{\text {nd }}$ degree polynomials (i.e. 3 parameters) yields the fit parameters as shown in Figure 7.9. The following tables are the parameters obtained from the fits. The ranges of detector $\eta$ for which

\footnotetext{
${ }^{5}$ The $b$ quark comes directly from the $t$ quark decay and is expectedly harder than the light quark which comes from the $W$ boson decay, which in turn come from the $t$ quark decay. Moreover, there is a significant difference in the detector's response to the light quark jets and the $b$ jets, which are dominated by the presence of semi-leptonic decays of b quarks.
} 
the corrections were established are displayed in Table 7.3. Additional information regarding this correction ${ }^{6}$ can be found in Appendix F. A detailed study can be found in [71].

${ }^{6}$ These corrections were obtained in Summer 2003. The corrections used in this dissertation have been updated. 


\begin{tabular}{lccc}
\hline pseudorapidity & $\mathrm{p}_{\mathbf{0}}(\mathrm{GeV})$ & $\mathrm{p}_{1}$ & $\mathrm{p}_{\mathbf{2}}\left(\mathrm{GeV}^{-\mathbf{1}}\right)$ \\
\hline$|\eta|<0.7$ & -2.927 & 0.9602 & $3.184 \times 10^{-4}$ \\
$0.7 \leq|\eta|<1.8$ & 3.847 & 0.8541 & $7.465 \times 10^{-4}$ \\
$1.8 \leq|\eta|<2.5$ & 11.340 & 0.8071 & $6.510 \times 10^{-4}$ \\
\hline
\end{tabular}

Table 7.6: Cocfficients for jets matched to light quarks, as a function of detector $\eta$.

\begin{tabular}{lccc}
\hline pscudorapidity & $\mathrm{p}_{\mathbf{0}}(\mathrm{GcV})$ & $\mathrm{p}_{1}$ & $\mathrm{p}_{\mathbf{2}}\left(\mathrm{GcV}^{-1}\right)$ \\
\hline$|\eta|<0.7$ & 0.2687 & 0.8600 & $5.333 \times 10^{-4}$ \\
$0.7 \leq|\eta|<1.8$ & 0.2231 & 0.8534 & $4.402 \times 10^{-4}$ \\
$1.8 \leq|\eta|<2.5$ & 4.328 & 0.7913 & $5.854 \times 10^{-4}$ \\
\hline
\end{tabular}

Table 7.7: Cocfficients for jets matched to $b$ quarks, as a function of detector $\eta$. Jets that contain a muon were corrected according to the Method described in[71].

\begin{tabular}{lccc}
\hline pseudorapidity & $\mathrm{p}_{\mathbf{0}}(\mathrm{GeV})$ & $\mathrm{p}_{1}$ & $\mathrm{p}_{\mathbf{2}}\left(\mathrm{GeV}^{-\mathbf{1}}\right)$ \\
\hline$|\eta|<0.7$ & -3.743 & 0.9291 & $2.719 \times 10^{-4}$ \\
$0.7 \leq|\eta|<1.8$ & -0.8011 & 0.8513 & $5.225 \times 10^{-1}$ \\
$1.8 \leq|\eta|<2.5$ & 19.37 & 0.6306 & $9.619 \times 10^{-4}$ \\
\hline
\end{tabular}

Table 7.8: Coefficients for jets without muon matched to $b$ quarks, as a function of detcctor $\eta$.

\begin{tabular}{lccc}
\hline pseudorapidity & $\mathrm{p}_{\mathbf{0}}(\mathrm{GeV})$ & $\mathrm{p}_{1}$ & $\mathrm{p}_{\mathbf{2}}\left(\mathrm{GeV}^{-\mathbf{1}}\right)$ \\
\hline$|\eta|<0.7$ & 34.09 & 0.5569 & $1.641 \times 10^{-4}$ \\
$0.7 \leq|\eta|<1.8$ & 52.21 & 0.3817 & $1.682 \times 10^{-4}$ \\
$1.8 \leq|\eta|<2.5$ & 0 & 0.60 & $1.1 \times 10^{-3}$ \\
\hline
\end{tabular}

Table 7.9: Coefficients for jets with muons matched to $b$ quarks, as a function of detector $\eta$. The jets were corrected according to the method described in[71]. In the forward region, enough data was not obtained to fit the low-energy behavior. Therefore, we were forced to set $p_{0}=0$ in the fit. 


\subsection{Electron energy resolution}

The energy resolution of the em-calorimeter $\sigma_{E_{c m}}$ is parameterized by

$$
\left(\frac{\sigma_{E_{c m}}}{E_{e m}}\right)^{2}=C_{e m}^{2}+\left(\frac{S_{e m}}{\sqrt{E_{e m}}}\right)^{2}+\left(\frac{N_{e m}}{E_{e m}}\right)^{2}
$$

Here $\sigma_{\dot{E}_{e m}}$ is the encrgy residual $E_{\text {true }}-E_{e m}$, where $E_{\text {true }}$ is the encrgy of the simulated electron, and $E_{e m}$ is the reconstructed energy, after application of oversmearing corrections to it. Parameters $C_{e m}, S_{e m}$, and $N_{e m}$ represent the constant term, the sampling term and the noise term for the em-calorimeter.

The noise term $\left(N_{e m}\right)$ accounts for the:

- the energy equivalent of the electronics noise,

- the fluctuation in energy due to pile up. In this case particles, other than those of interest cause the the encrgy fluctuations.

The sampling term $\left(S_{e m}\right)$, also known as stochastic term, accounts for:

- the statistical fluctuations in the number of primary processes.

The constant $\left(C_{e m}\right)$ term accounts for contributions from:

- physical imperficctions in the calorimeter matcrial.

- non-unilormity of signal gencration and/or collection,

- ccll-to-ccll intcrcalibration crror(s),

- fluctuations in the amount of encrgy lcakage from the periphery of the matcrial,

- losses in dead regions of the detector. These regions cannot be read out due to some mechanical failure. 
- contributions from the fluctuation in the em component in the hadronic showers.

After the scale and oversmearing corrections described in Section 7.1 have been applied to simulated events, the variance of the electron energy residuals are cvaluated in definite $\Delta E_{\text {true }}$ intervals. The distribution of the variance evaluated from the residuals are plotted versus the $\Delta E_{\text {true }}$ intervals in Figures 7.10 and 7.11 . The distribution is fit to the function shown in Fquation 7.6. The parameters from the best numerical fit to the distribution determines the detector's energy resolution parameters for the high- $E_{T}$ clectrons. $\Lambda$ these energy scales, the noise term is negligible compared to the contributions of the sampling and the constant terms. Table $7.10[66]$ shows the values for the clectron cnergy resolution parameters.

\begin{tabular}{|l|l|l|l|}
\hline $\begin{array}{l}\text { detector } \\
\text { region }\end{array}$ & $\begin{array}{l}\mathrm{C}_{\mathrm{em}} \\
\text { parameter }\end{array}$ & $\begin{array}{l}\mathrm{S}_{\mathrm{em}} \\
\text { parameter } \\
(\sqrt{\mathrm{GcV}})\end{array}$ & $\begin{array}{l}\mathrm{N}_{\mathrm{em}} \\
\text { parameter } \\
(\mathrm{GcV})\end{array}$ \\
\hline (CC) - within fiducial & $0.0439 \pm 0.0002$ & $0.221 \pm 0.002$ & - \\
(CC) - not in fiducial & $0.1116 \pm 0.0011$ & $0.385 \pm 0.013$ & - \\
(FC) & $0.0316 \pm 0.0005$ & $0.258 \pm 0.006$ & - \\
\hline
\end{tabular}

Table 7.10: Energy resolution parameters for the central calorimeter (CC) and end calorimeter (EC) as a function of $\eta_{\text {del }}$. 

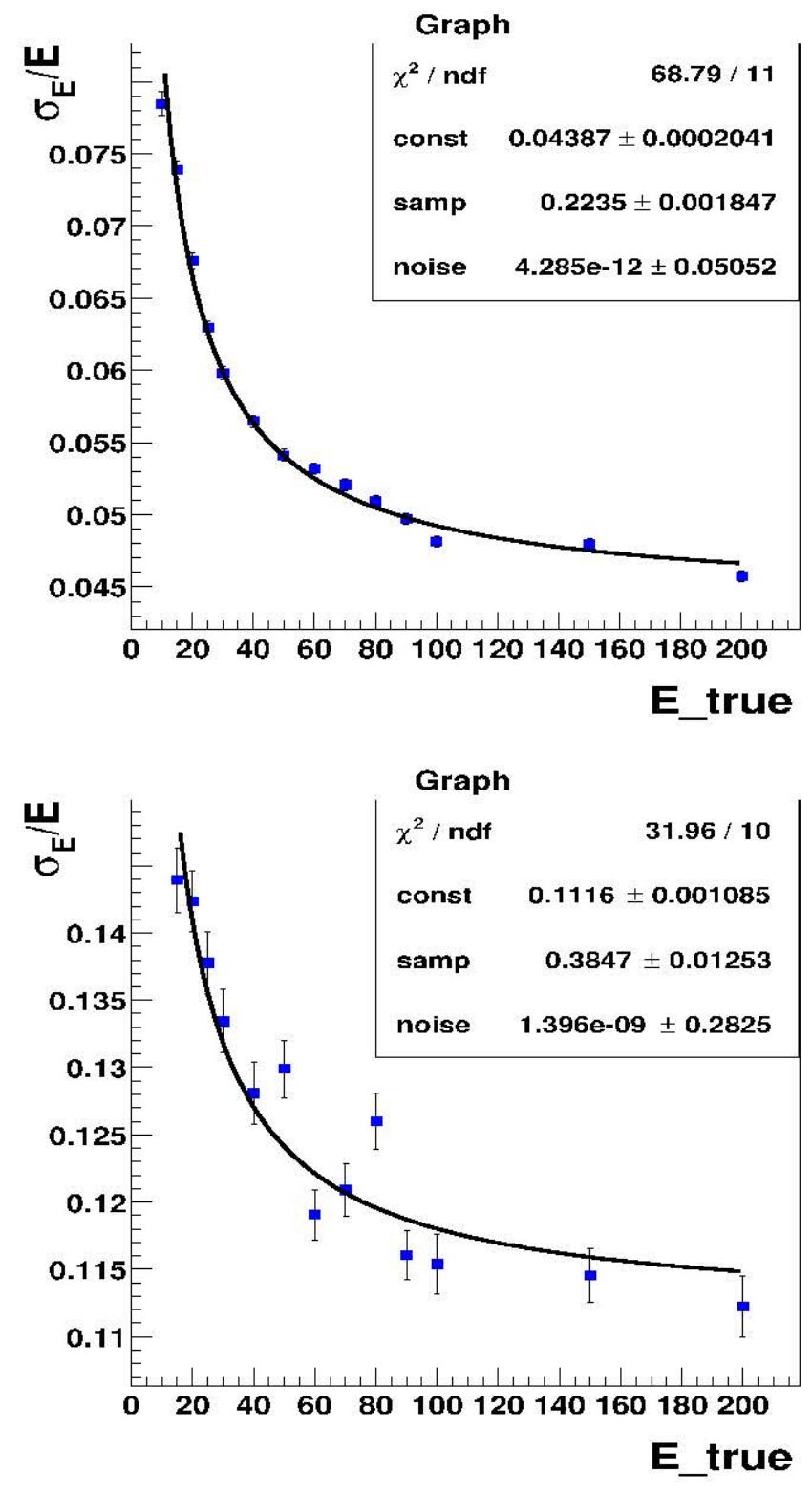

Figure 7.10: The distribuion of the fractional electron energy resolution versus electron energy in the central calorimeter. The best fit to the distribution yields emcalorimeter resolution parameters for CC em-calorimeter. The top plot represents the case when both the electron objects used in reconstructing the $Z$ resonance are in the CC. The bottom plot represents the case when one of the clectrons is not in the CC. The plots are obtained from S. Jain [66]. 


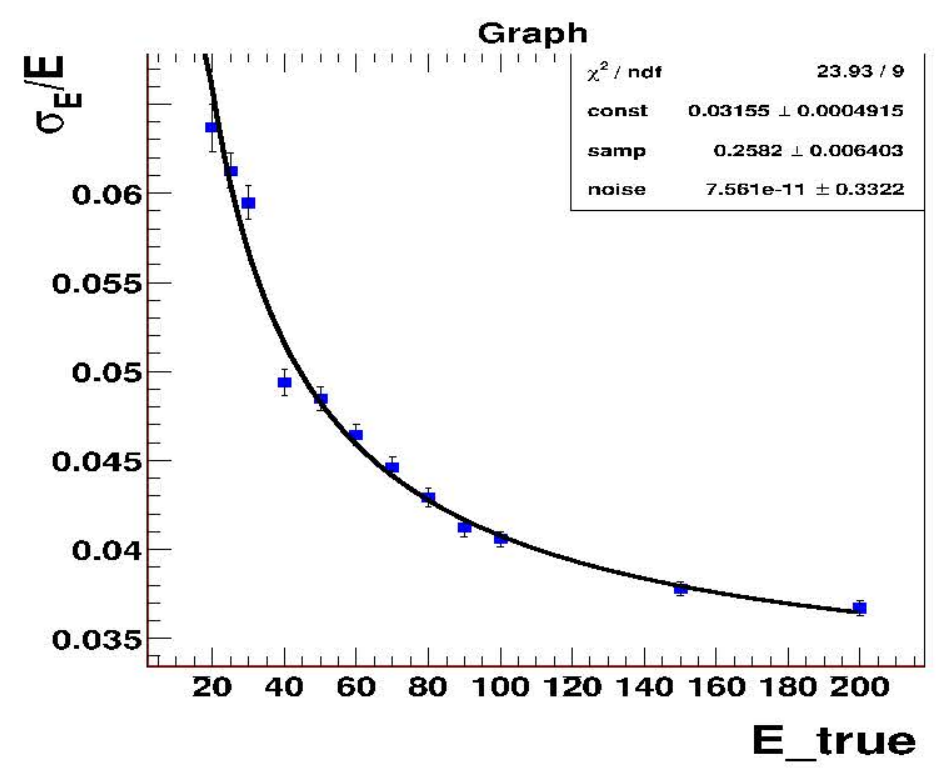

Figure 7.11: The distribution of the fractional electron cnergy resolution versus clectron encrgy in the end-cap calorimeters. The parametcrs from the best fit to the distribution yiclds the resolution parametcrs for end cap elcetromagnetic calorimeter. This plot is obtained with both the electron objects used in reconstructing the $Z$ resonance in the end calorimeters. The plot is obtained from S. Jain [66]. 


\subsection{Muon momentum resolution}

The parameterization of the muon transverse momentum $\left(p_{T}\right)$ resolution is given $\operatorname{as}[68]$

$$
\sigma_{1 / p_{T}}^{2}=\left\langle\left(\frac{1}{p_{T}^{M C}}-\frac{1}{p_{T}}\right)^{2}\right\rangle=a^{2}+\left(\frac{b}{p_{T}}\right)^{2} .
$$

This parameteric representation is motivated by the inherent tracking resolution of a charged particle in a magnetic field and by the multiple scattering of the charged particle in the detector volume. After the transverse monentum resolution is obtained, the momentum resolution is corrected as a function of the transverse momentum resolution and the polar angle resolution.

For evaluating the resolution, muon objects which have the scaling and oversmcaring corrections applied to them. The residual of the inverse transverse momentum is estimated as a function of the inverse muon $p_{T}$. Then Gaussian fits to the distribution are used to estimate the variance $\left(\sigma_{1 / p_{T}}\right)$ of the residual is obtained

for intervals in $\Delta_{1 / p^{\prime}}$ as shown in Figure 7.12. The $\sigma_{1 / p_{T}}$ distribution as a function of $1 / p_{T}$ is parameterized using Equation 7.7. The best values of parameters $a$ and $b$ from numerical fits are used as the resolution parameters. Figures 7.13 and 7.14 illustrate $\sigma_{p_{T}}$ of the muons as a function of their inverse $p_{T}$ for the central and the forward regions respectively. The resolution $\sigma_{p_{T}}$ thus obtained is for the muon system in conjunction with the tracking system. Further details of this analysis are described by D. Shpakov [67]. 


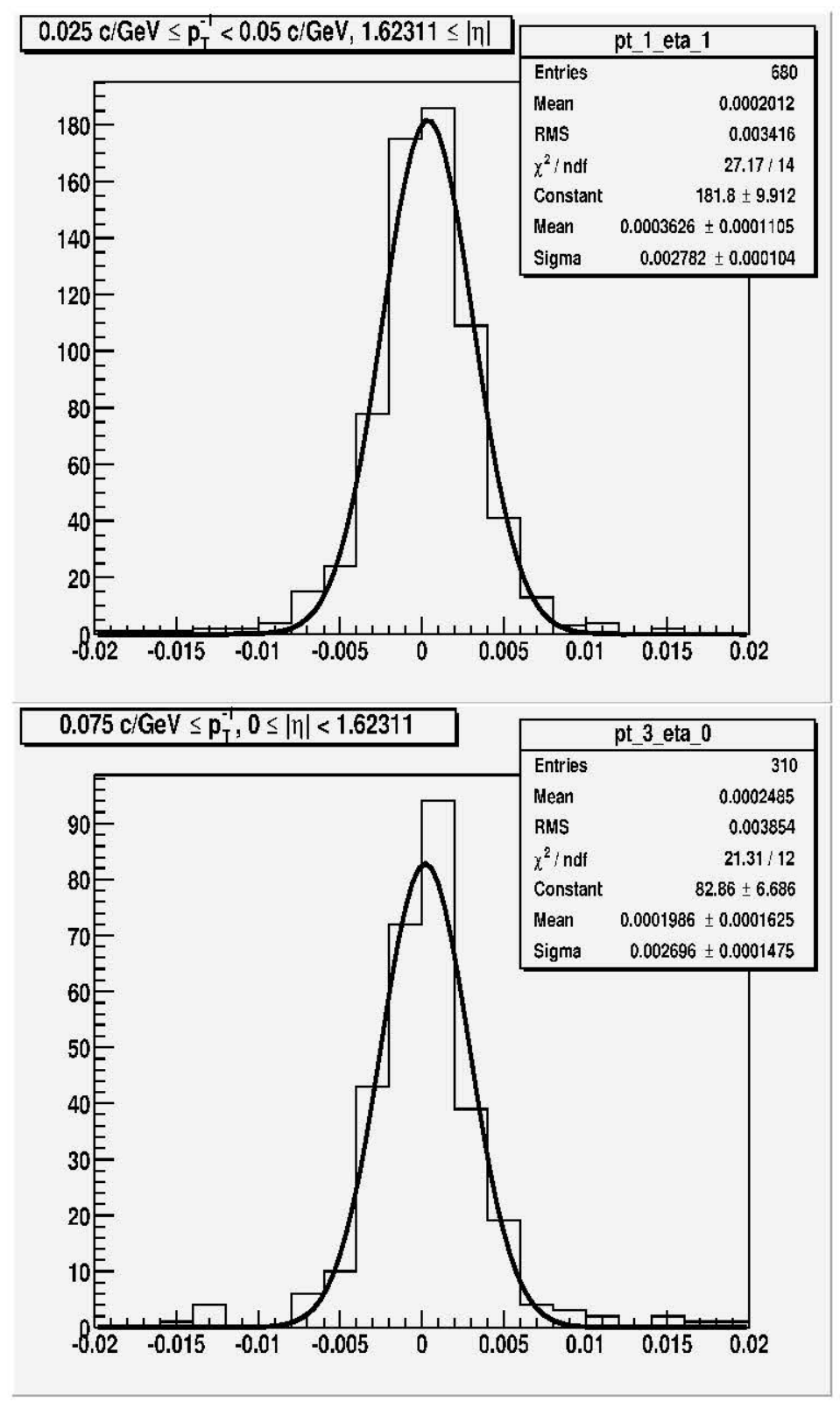

Figure 7.12: Muon inverse $p_{T}$ residuals for some $\Delta p_{T}$ and $\Delta \eta$ ranges. The variance of best fits from these distributions give a measure of the $\sigma_{1 / p_{T}}$ for various $1 / p_{T}$ intervals. These values are then used to estimate the inverse transverse momentum resolution parameters. Above plots are obtained from D. Shpakov [67]. 


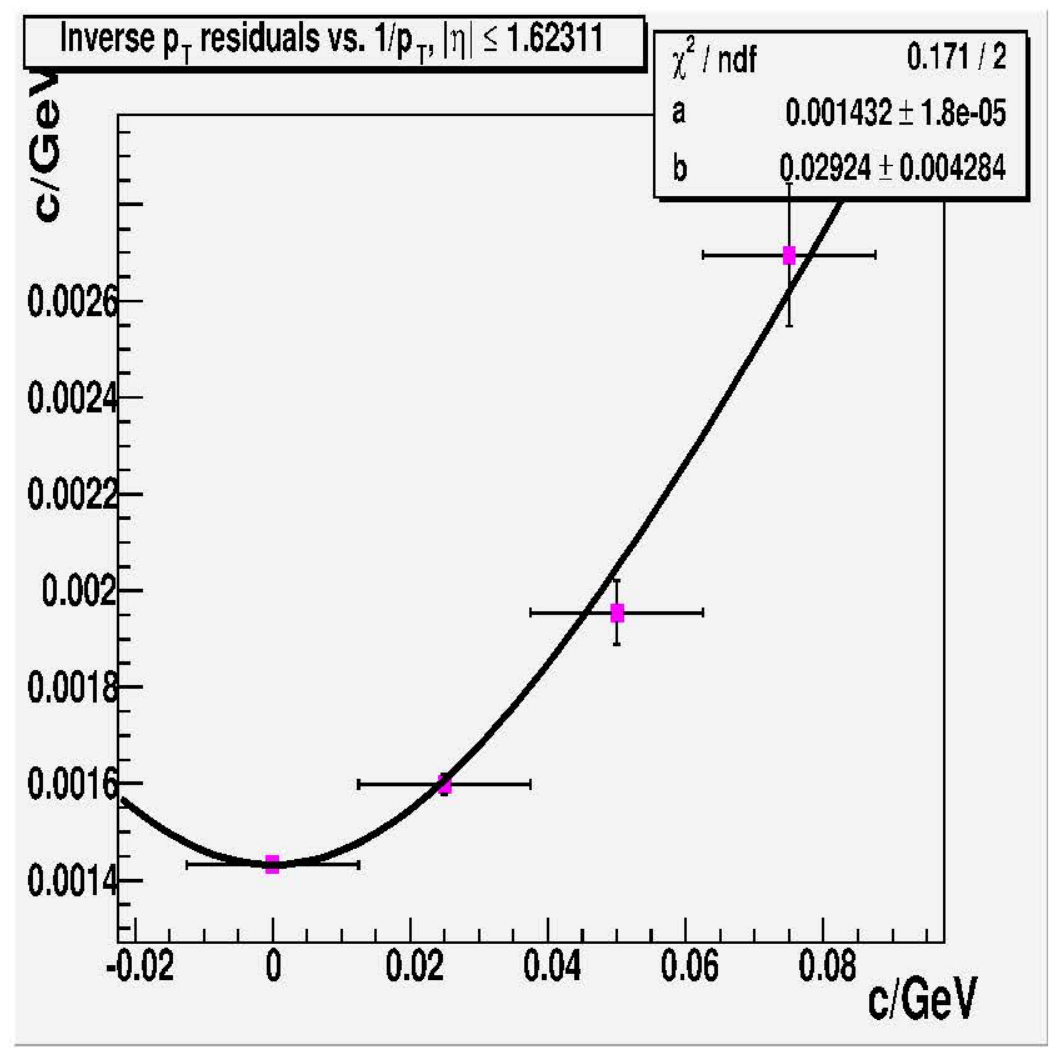

Figure 7.13: $\sigma_{1 / p_{T}}$ of the muons as a function of $1 / p_{T}$ for the central region. The best fits to residual distributions yield the values of $\sigma_{p_{T}}$ used. Muon resolution parameters from the central muon system as a function of the muon $1 / p_{T}$. The horizontal crror bar corresponds to the bin width. This figure is obtained from D. Shpakov[67]. 


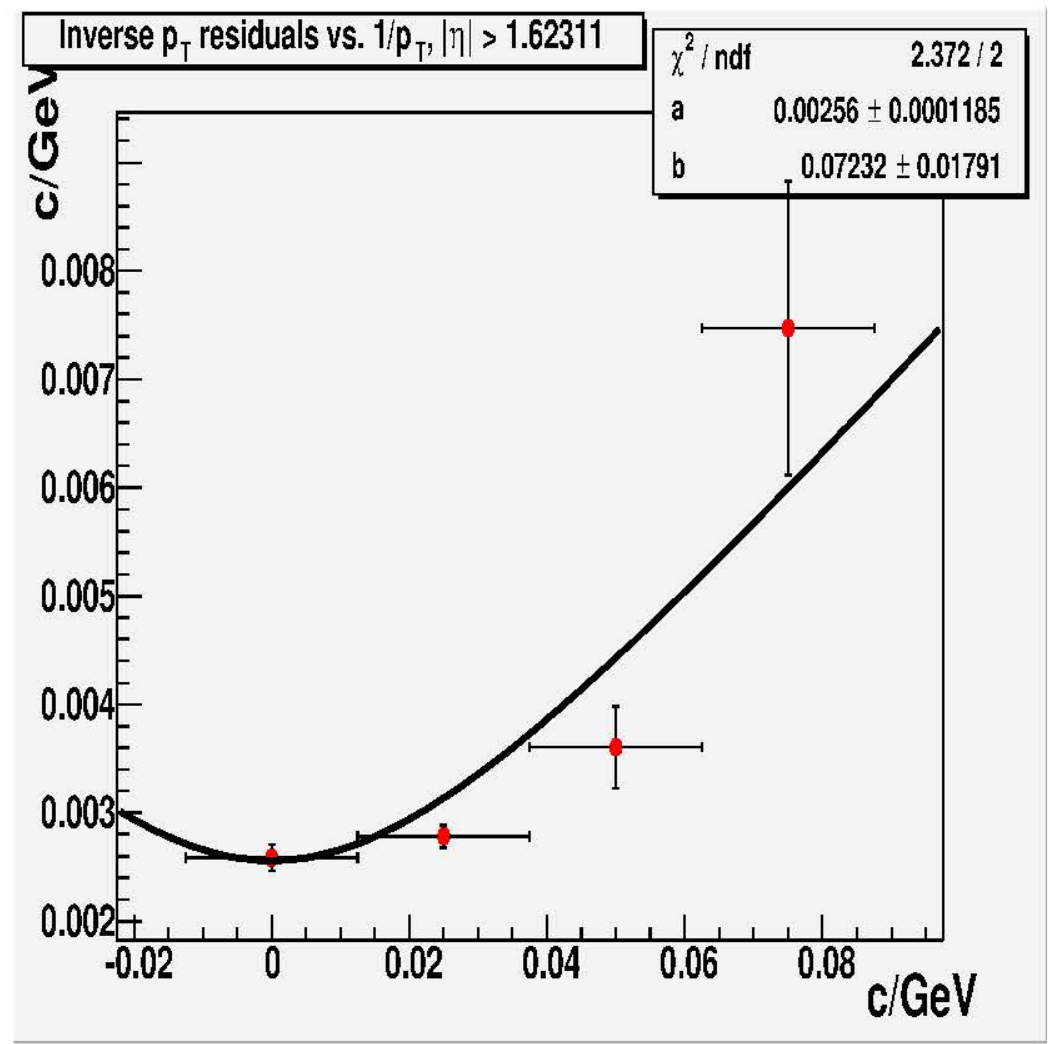

Figure 7.14: $\sigma_{1 / p_{T}}$ of the muons as a function of $1 / p_{T}$ for the forward region. Muon resolution parameters from the forward muon system as a function of the muon $1 / p_{T}$. The horizontal crror bar corresponds to the bin width. This figure is obtained from D. Shpakov [67]. 


\subsection{Jet energy resolution}

Similar to the case of electron energy, the energy resolution of a jet of energy $E$ is parameterized as

$$
\left(\frac{\sigma_{E}}{E}\right)^{2}=C^{2}+\left(\frac{S}{\sqrt{E}}\right)^{2}+\left(\frac{N}{E}\right)^{2}
$$

where $C, S$, and $N$ represent the constant term, the sampling term and the noise term for the calorimeter.

Di-jet events are used to estimate the residual transverse energy as a function of the mean transverse energy. If the calorimeter were ideal in its response, then the vector sum of the total transverse encrgy would be a null value for the di-jet events. The jet cone algorithms have an ad-hoc cut of $8.0 \mathrm{GcV}$ for the L1 $E_{T}$ which may bias results for the jet $E_{T}$ calibration. Morcover the jet turn on curve as a function of offline $E_{T}$ is much more sluggish than that of the electron $E_{T}$. These factors motivate the establishment of the jet transverse energy resolution as a function of $E_{T}$ for $E_{T} \geq 50.0 \mathrm{GoV}$ using di-jet events. For the range $E_{T}<50.0 \mathrm{GoV}$, cvents with $\gamma+$ jet objects are used to evaluate the residual $E_{T}$. Once this is accomplished, then the variance from fits to residuals are obtained as a function of a fixed range of $E_{T}$. This is then established for various valucs of $E_{T}$. The best fit to the distribution, such as one in Figure 7.15 yields the resolution parameters in Equation 7.8. Instead of using the encrgy variable, the di-jet invariant mass is used as a representative variable. 


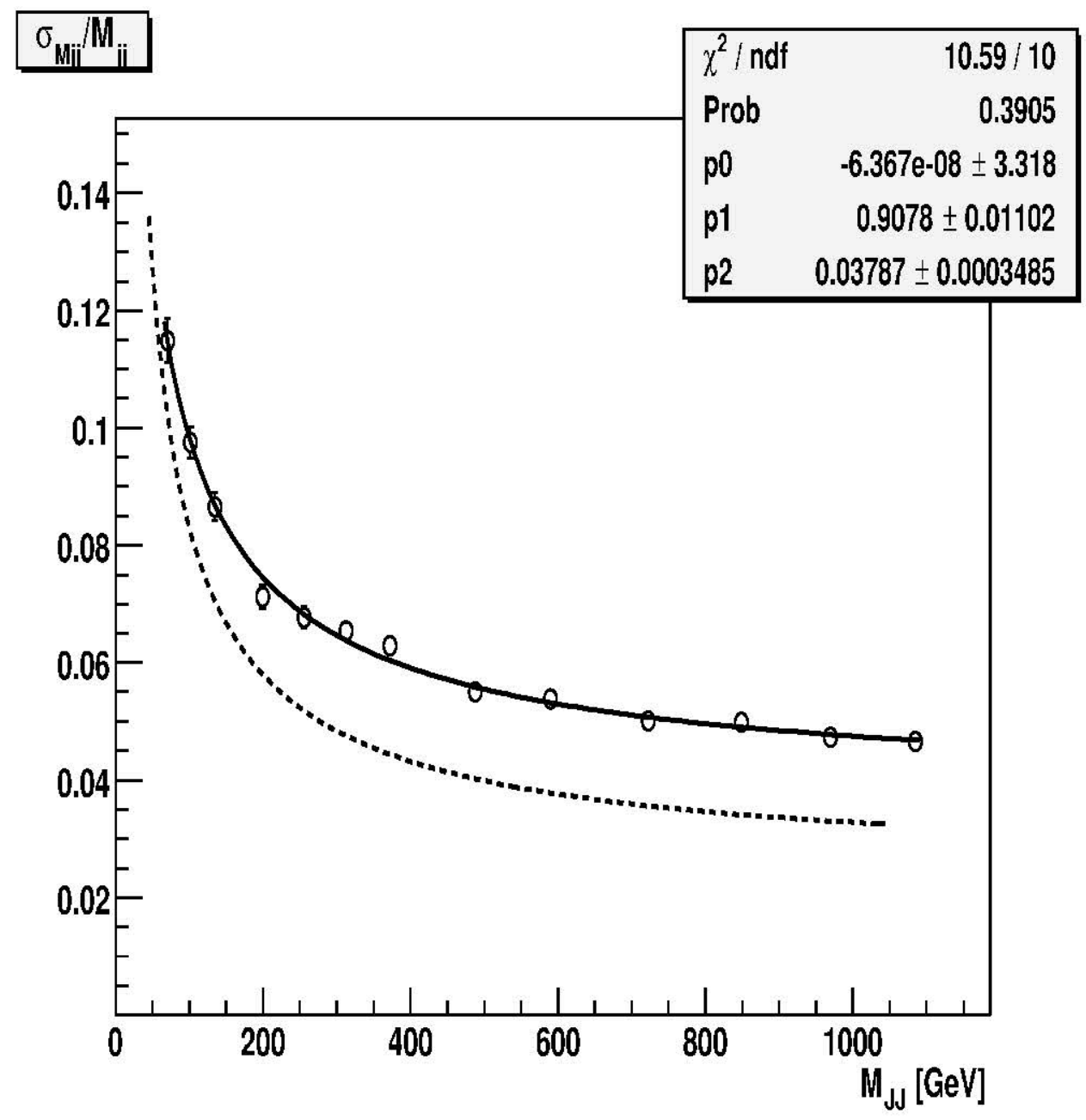

Figure 7.15: Parameteric representation of the resolution of jet energy scale. The solid line represents the fit to data distribution, while the dashed line represents the fit to events obtained via Monte Carlo simulation. The values of $p 0, p 1, p 2$ obtained from the best numerical fit correspond to the parameters obtained from data events for the constant term $C$, the sampling term $S$ and the noise term $N$. The dotted curve represents the Run I parametcric curve. This plot is obtained from A. Kupco[69]. 


\subsection{Summary of the object resolutions}

For the selected data events and the standard IDØ Monte Carlo events, the measured transverse momenta of objects are smeared with their experimental resolutions. For the analysis on mass measurement, the resolutions ${ }^{7}$ used are listed in the table below:

\begin{tabular}{l|r|r}
\hline$|\eta|$ region & $\mathbf{a}\left(\mathrm{GeV}^{-2}\right)$ & $\mathbf{b}$ \\
\hline$|\eta|<1.6$ & 0.00276 & 0.0279 \\
$|\eta|>1.6$ & 0.00522 & 0.0479 \\
\hline
\end{tabular}

Table 7.11: The parameterization of resolution for reconstructed muons.

\begin{tabular}{l|r|r|r}
\hline$|\eta|$ region & $\mathbf{N}_{\mathrm{cm}} / \mathrm{GcV}^{2}$ & $\mathbf{S}_{\mathrm{cm}} / \mathrm{GcV}$ & $\mathbf{C}_{\mathrm{cm}}$ \\
\hline$|\eta|<1.1$ & 0.21 & 0.23 & 0.041 \\
$1.5<|\eta|<2.5$ & 0.20 & 0.26 & 0.032 \\
\hline
\end{tabular}

Table 7.12: The parametcrization of resolution for reconstructed electrons. These numbers have becn obtaincd from the reference

\begin{tabular}{l|r|r|r}
\hline$|\eta|$ region & $\mathbf{N} / \mathrm{GeV}^{2}$ & $\mathbf{S} / \mathrm{GeV}$ & $\mathbf{C}$ \\
\hline$|\eta|<0.5$ & 5.05 & 0.753 & 0.089 \\
$0.5 \leq|\eta|<1.0$ & 0.00 & 1.2 & 0.087 \\
$1.0 \leq|\eta|<1.5$ & 2.24 & 0.924 & 0.135 \\
$|\eta| \geq 1.5$ & 6.42 & 0.0 & 0.097 \\
\hline
\end{tabular}

Table 7.13: The parametcrization of resolution for reconstructed jets.

\footnotetext{
${ }^{7}$ These standard resolutions parameters were obtained from the Top Quark Properties Group in spring 2004.
} 


\section{Chapter 8}

\section{Mass Measurement}

Until now we have described the various steps taken and the tools used to sclect a set of events which represent the characteristics of top and anti-top quark pairs decaying into the di-lepton channel. This chapter describes a method for determining the mass of the top quark in the di-lepton channel using the selected events.

In order to illustrate the complexity of the problem, the di-lepton event topology is first described, and specific measurements from the selected event are obtained. After a description of the problem a solution is illustrated. Detailed studies involving the application of the method to simulated events for performing self-consistency tests as well as establishing the associated systematic unccrtaintics are shown. $\Lambda$ measurement of the mass of top quark from Tevatron data is obtained, fulfilling the goal of this thesis.

\subsection{The di-lepton event topology}

In the di-lepton channel top anti-top quark pairs decay via $t \rightarrow W b$, followed by $W \rightarrow l \nu_{l}$ yielding six final-state particles as displayed in Figure 8.1. These final-state 


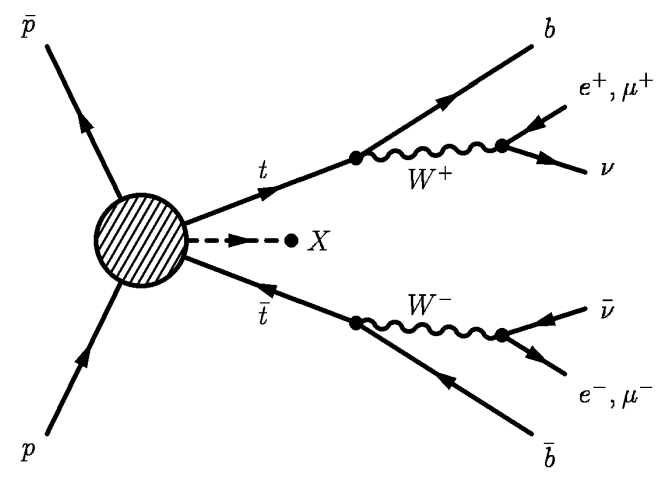

Figure 8.1: A schematic of the di-lepton event topology: $p \bar{p} \rightarrow t \bar{t}+X \rightarrow$ $b l_{1}^{+} \nu_{l_{1}} \bar{b} l_{2}^{-} \bar{\nu}_{l_{2}}+X$. The six particle final-state is the simplest case with two $b$ jets in the event.

particles are:

- A pair of charged leptons from ${ }^{1}$ :

$$
\left(e^{+}, e^{-}\right),\left(\mu^{+}, \mu^{-}\right),\left(e^{+}, \mu^{-}\right) \text {or }\left(e^{-}, \mu^{+}\right) \text {. }
$$

- The corresponding pair of neutrinos from among:

$\left(\nu_{e}, \bar{\nu}_{e}\right),\left(\nu_{\mu}, \bar{\nu}_{\mu}\right),\left(\nu_{e}, \bar{\nu}_{\mu}\right)$, or $\left(\nu_{e}, \bar{\nu}_{\mu}\right)$.

- The $b, \bar{b}$ jets.

However, there may be additional jets in the event from initial-state radiation, finalstate radiation or from split jets. In this analysis only the two leading transverse momentum jets in the event are considered. If there are additional jets in the event then they are neglected.

If the identities as well as the 3-momenta of the final-state particles are known (18 quantities), then the complete event reconstruction is possible. However, we can only identify and measure the 4-momenta of the jets and the charged leptons. The two neutrinos in the event remain undetected, but the vector sum of their transverse

\footnotetext{
${ }^{1}$ As explained before, this analysis does not consider the final-state with $\tau, \nu_{\tau}$
} 
momenta can be inferred from the observed missing $p_{T}$ in the event. Therelore, a set of only 14 observables $\{o\}$ out of the 18 values $\{v\}$ are measured.

In order to constrain the $l \bar{l}$ event kinematics the energy-momentum conservation principle is imposed. For completeness, a description of the algorithm proposed by Dalitz and Goldstein in reference[72],[73] is presented in the next sub-section.

\subsubsection{Constraints from the event topology}

This sub-section describes the mathematical construct for the di-lepton event analysis. Consider $\vec{t}, \vec{b}$ and $\vec{l}$ to represent the 3 -momenta for the $t, b$ and $l$ final-state particles in the laboratory frame of reference, while $t^{\alpha}, b^{\alpha}$, and $l^{\alpha}$ are the corresponding covariant 4-momenta in the same frame of reference. Since the neutrino is undetected, all constraints are expressed in terms of $l^{\alpha}, b^{\alpha}$, and $l^{\alpha}$. Herc $b^{\alpha}$, and $l^{\alpha}$ are the measured quantities, and $t^{\alpha}$ is the quantity we seek. Using energymomentum conservation, we obtain three sets of constraints:

A. The invariant mass of the charged lepton and its corresponding neutrino is set to be equal to the mass of the $W$ boson, $m_{W}$. Two independent constraints are obtained for the charged lepton and its corresponding anti-ncutrino and the charged anti-lepton and its corresponding neutrino. The Lorentz invariant equation for the particle pair is:

$$
\left(t^{\alpha}-b^{\alpha}\right) \cdot\left(t_{\alpha}-b_{\alpha}\right)=\left(E_{t}-F_{b}\right)^{2}-(\vec{t}-\vec{b})^{2} \equiv M_{W}^{2}
$$

In order to solve for $t^{\alpha}$, this equation can be re-written as:

$$
(\vec{t}-\vec{b})^{2}=\left(E_{t}-E_{b}\right)^{2}-M_{W}^{2}=R_{W}^{2}(\text { say })
$$


We obtain an equation similar in form for the anti-particle system.

B. The invariant mass of the particles from the decay of the top quark is set to be the invariant mass of all the anti-particles which decay from the anti-top quark. This can be set in terms of the detected observables as:

$$
\left(t^{\alpha}-b^{\alpha}-l^{\alpha}\right) \cdot\left(t_{\alpha}-b_{\alpha}-l_{\alpha}\right)=\left(E_{t}-E_{b}-E_{l}\right)^{2}-(\vec{t}-\vec{b}-\vec{l})^{2} \equiv m_{\nu}^{2}
$$

The mass of the neutrino $\left(m_{\nu}\right)$ is neglected. Therefore, we obtain:

$$
\left(E_{t}-E_{b}-E_{l}\right)^{2}=(\vec{t}-\vec{b}-\vec{l})^{2}=R_{\nu}^{2}(\text { say })
$$

Incorporating Equations 8.1 and 8.2, the event kinematics remain under-constrained by just one equation. In this thesis we use a hypothesized value of the mass of the top quark to fully constrain the set of kinematic variables from the event.

Let us first consider the system of the intermediate state particle the t-quark, and the final-state particles $b$-quark and the charged lepton $l$. When Equations 8.1 and 8.2 have common solutions for the 1 -momentum $t^{\alpha}$ in the laboratory frame of reference, then the kinematic configuration yields a set of solutions for the neutrino momentum as well. We now illustrate that multiple solutions may exist for the noutrino and anti-ncutrino momenta.

Consider the schematic shown in Figure 8.2. From the origin at point $\mathrm{P}$, the 3-momenta $\vec{b}(\overrightarrow{P B})$ and $\vec{l}(\overrightarrow{B L})$ are illustrated in succession. Point $B$ is the center of a sphere of radius $R_{W}$ described by Equation 8.1, and the point $L$ is the center of the sphere described by Equation 8.2 with radius $R_{\nu}$. In order to obtain realistic solutions in the 3 particle decay scheme $l \rightarrow b W \rightarrow b l \nu_{l}$, these spheres must intersect. The momentum vector $\vec{t}$ is a valid solution for the Equations 8.1 and 8.2 if it lies on 


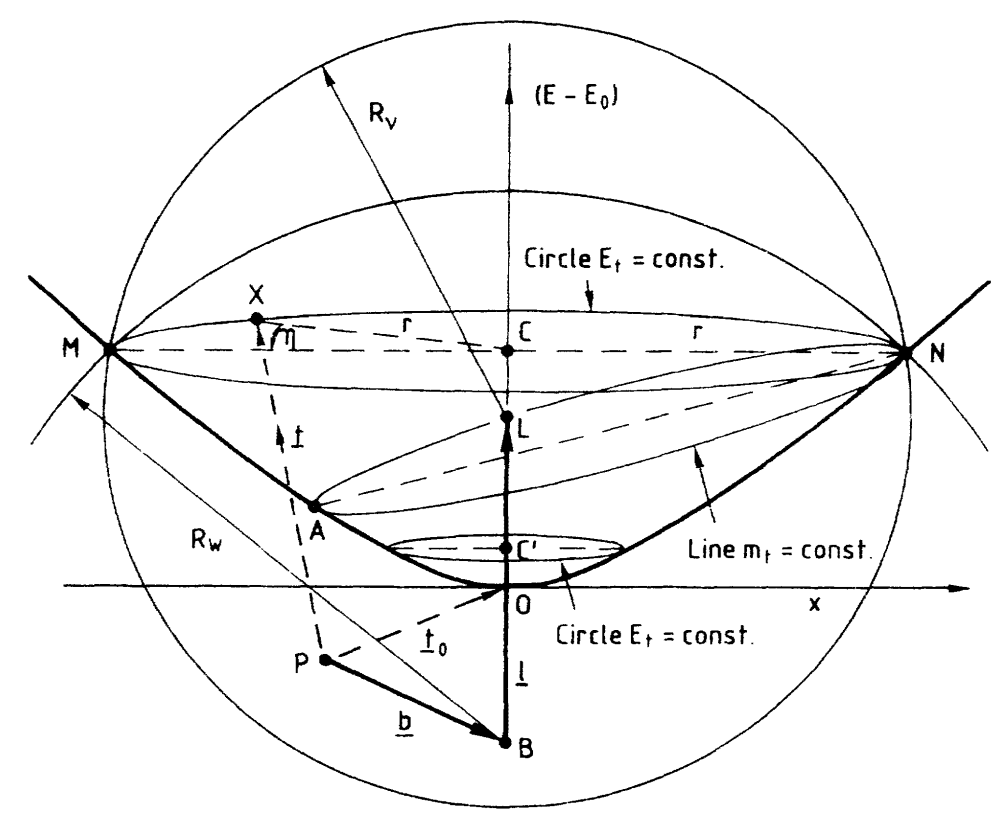

Figure 8.2: A representation of the 3-momentum vectors in the lab frame of reference for the $t \rightarrow b W, W \rightarrow l \nu_{l}$ decay sequence. This diagram is from [73].

the circle of intersection of the two spheres. This circle of intersection is represented along $M N$, the solid line in Figure 8.2, or the dashed line $M N$ in Figure 8.3. The radius of this circle of intersection $\overrightarrow{C X}=\vec{r}$, is given in the reference by Dalitz and Goldstein[72],[73]:

$$
|\vec{r}|^{2}=\frac{M_{W}^{2}}{E_{l}}\left(E_{t}-E_{0}\right)
$$

where,

$$
E_{0}=E_{b}+E_{l}+\frac{M_{W}^{2}}{4 E_{l}}
$$

is the minimum value of $E_{t}$ which can yield physical solutions[72],[73]. For a range of values of $|\vec{b}|$ and $|\vec{l}|$, spheres of varying $|\vec{r}|$ will be obtained. It can be established[73] that all such circles can be enveloped by a paraboloid as shown in Figure 8.3. All 


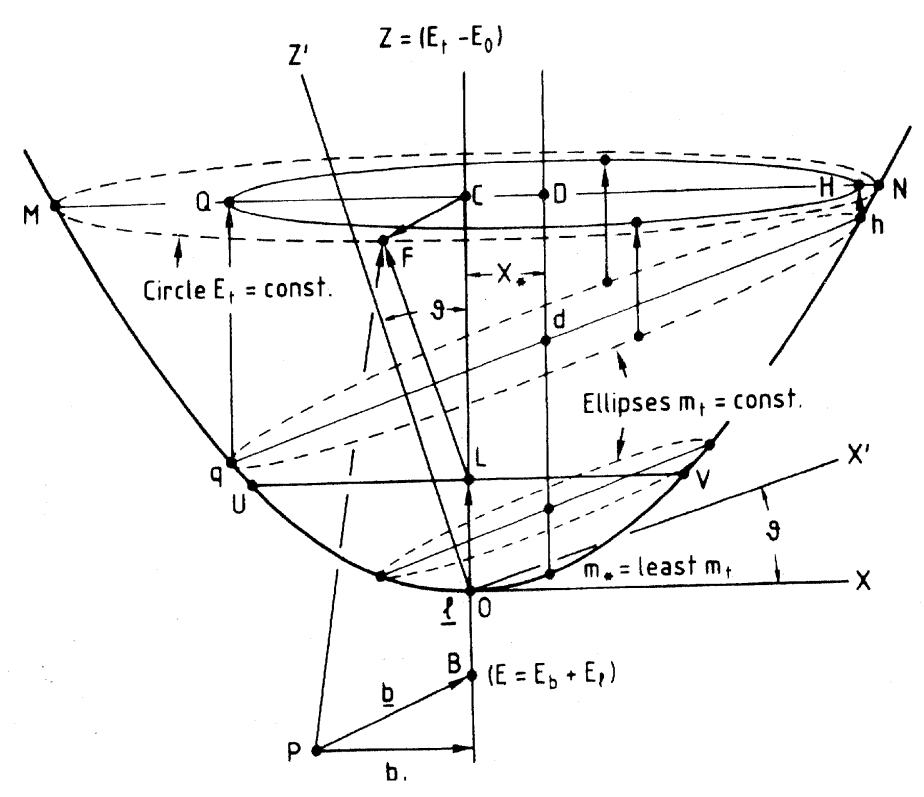

Figure 8.3: The geometrical representation of the paraboloid surface for all values of the momenta $\vec{t}$ which can be established to be physically consistent with the observed values of $\vec{b}$ and $\vec{l}$. This diagram is from [72], and is a close-up of the paraboloid shape from Figure 8.2.

possible values of $\vec{t}$ lie on the surface of the paraboloid. A convenient parametric form of representing the equation of the paraboloid is:

$$
\vec{t}=\overrightarrow{t_{0}}+\left(E-E_{0}\right) \hat{l}+\hat{i} r \cos \eta+\hat{j} r \sin \eta
$$

where $\hat{j}=\hat{b} \times \hat{l}, \hat{i}=\hat{l} \times \hat{j}$ and the angle $\eta$ is subtended by $\overrightarrow{C X}$ and $\hat{i}$. Moreover, the parameter $\overrightarrow{t_{0}}$ is the top quark momentum at the bottom of the paraboloid when $E=E_{0}$, and it is given by[72],[73]:

$$
\overrightarrow{t_{0}}=\vec{b}+\vec{l}\left(1-\frac{M_{W}^{2}}{4 E_{l}^{2}}\right)
$$

Although the equation of the circle $M N$ provides solutions for $\vec{t}$ pertaining to 
the event, these solutions do not correspond to a constant value of $m_{l}$. It can be proved $[72],[73]$ that points having the same value of $m_{t}$ lie on the plane defined by unit vectors $\hat{l}$ and $\hat{i}$, where $\hat{i}=\hat{l} \times(\hat{b} \times \hat{l})$. Intersection of constant $m_{l}$ planes and the paraboloid results in the slanted ellipse with the major axis given by the line segment $\overline{q h}$ in Figure 8.3. Therefore, the vector $\vec{t}$ described by such ellipses is consistent with the decay kincmatics. The projection of this cllipse on to the original plane $M N$, which is perpendicular to $\vec{l}$, gives a circle with $Q H$ as its diameter and centered at $D$, as shown in Figure 8.3. The radius $r_{\star}$ of this circle is given by [72],[73]:

$$
r_{\star}^{2}=\frac{M_{W}^{2}}{2 b_{\alpha} l^{\alpha}}\left(m_{t}^{2}-m n_{\star}^{2}\right)
$$

In the above equation,

$$
m_{\star}^{2}=\left(m_{b}^{2}+2 b_{\alpha} l^{\alpha}\right) \cdot\left(M_{W}^{2}+2 b_{\alpha} l^{\alpha}\right) /\left(2 b_{\alpha} l^{\alpha}\right)
$$

and it represents the smallest possible value of $m_{t}$ which can be accommodated on the paraboloid surface. The top quark momcntum for this configuration can be parameterized on the circle, in terms of $\sigma$, the analog of $\eta$ in Equation 8.5,

$$
\vec{t}=\overrightarrow{t_{0}}+\hat{i} x_{\star}+\hat{l}\left(E-E_{0}\right)+\hat{i} r_{\star} \cos \sigma+\hat{j} r_{\star} \sin \sigma
$$

To reduce the mathematical complexity of the system, we project the circle on to the transverse momenta plane. The projection of $\vec{t}$ lies on an ellipse $A N$ on this transverse plane as shown in Figure 8.4. For constraining the six particle final-state 
we can define $\vec{p}_{T}(l \bar{l})$ as:

$$
\vec{p}_{T}(t \bar{t})=\vec{t}_{T}+\vec{t}_{T}=\vec{b}_{T}+\vec{l}_{T}^{-}+\vec{b}_{T}+\vec{l}_{T}^{+}+{\overrightarrow{p_{T}}}
$$

The vector $\vec{p}_{T}-\overrightarrow{\vec{l}}_{T}$ lies on the transverse plane and is related to $\overrightarrow{\vec{l}}_{T}$ by a rellection at the origin and a translation. The locus of all $\vec{t}$ solutions lie on an ellipse $A N^{\prime}$ on this plane as shown in Figure 8.4. When the two ellipses $A N$ and $A N^{\prime}$ intersect then physical solutions for the transverse momenta of the neutrino (in the laboratory frame of reference) are obtained. Both ellipses are projected onto the transverse momenta plane, which is illustrated in Figure 8.1.

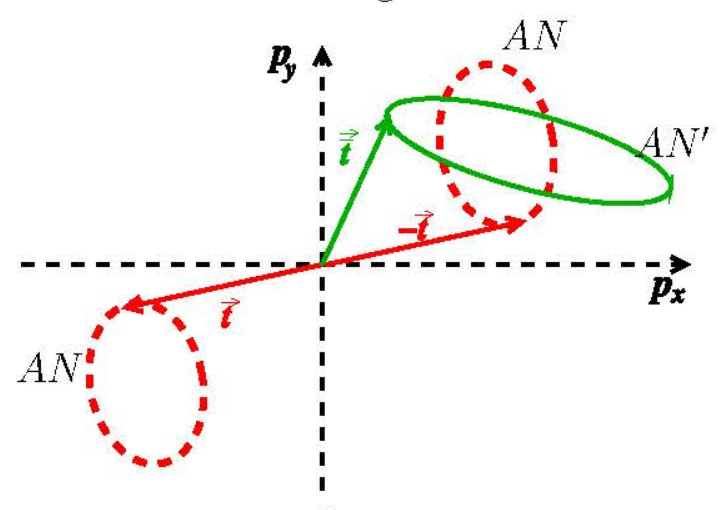

Figure 8.4: Solutions for the $\vec{t}$ and $\vec{t}$ projected on to the laboratory transverse monentum plane. The dotted and continuous ellipses represent the two independent projections (which are described by $A N$ and $A N^{\prime}$ in this section) of $\vec{t}$ and $\vec{t}$.

The points where the two ellipses intersect correspond to a physical solution for $\vec{t}, \vec{t}$ of the top anti-top system in the laboratory reference frame. Therefore, there are 0,2 , or at most 4 solutions for a given $m_{t}$, for the neutrino and anti-neutrino momenta in the event ${ }^{2}$.

\footnotetext{
${ }^{2}$ Here we hypothetically distinguish the pairs of particles $b, l^{+}$from the anti-particles $\bar{b}, l^{-}$. By considering all sets of jet and charged lepton pairs this ambiguity is avoided.
} 


\subsection{The Method of analysis}

From the analyzed set of data, which corresponds to an integrated luminosity

of nearly $230 \mathrm{pb}^{-1}$, eight candidate events in the $e \mu$ channel and five candidate events in the di-electron channel have been obtained. No event passed the selection criterion for the di-muon channel[52]. These selected events are used to estimate the mass of the top quark. In this section, the mass of the top quark is treated as an unknown parameter. The Maximum Likelihood method[74] is used to estimate this unknown parameter.

First, individual candidate events are used to extract kinematic information. The event selection and the calibration of the 4 -vectors of objects was described in Chapters 6 and 7 respectively. In the next sub-section, we discuss the mass analysis of a single event. Finally, information from all candidate events is combined to estimate the most likely value of the mass of the top quark.

\subsubsection{The mass analysis of an event}

A hypothetical value of the top quark mass $m_{t}$ is used to solve the system of underconstrained equations. Then, for every event, up to $n$ real solutions are obtaincd for the neutrino and anti-neutrino momenta, where $n=0,2$ or 4 . There is a two-fold combinatoric ambiguity in pairing a charged lepton and a $b$-jet. I'herefore, up to $n=8$ possible neutrino momentum solutions are possible.

This algorithm was developed for measurement performed in Run I[27] $]^{3}$. Ideally we would like to calculate the probability to measure the 11 observables $\{o\}$, given

\footnotetext{
${ }^{3} \mathrm{~A}$ stand-alone software was prepared by appropriate modifications of the Run I code.
} 
the top quark mass $m_{l}$. This probability $p\left(\{o\} \mid m_{l}\right)$ can be expressed as:

$$
p\left(\{0\} \mid m_{t}\right)=\int_{\{v\}} d^{18}\{v\} \cdot p(\{o\} \mid\{v\}) \cdot p\left(\{v\} \mid m_{t}\right) .
$$

For every event $p(\{o\} \mid\{v\})$ is the probability density to measure the set of 11 observables $\{o\}$, for a given set of 18 final-state values $\{v\}$. It can be described by generating many signal events having identical input $m_{t}$. The $p\left(\{v\} \mid m_{t}\right)$ is the probability density to obtain the final-state set $\{v\}$ for a given $m_{t}$, which can be cvaluated using Feynman rules[75]. This can be simplified as:

$$
p\left(\{v\} \mid m_{l}\right)=d x d x|\mathcal{M}|^{2} f(x) f(x) .
$$

Here $f(x)$ and $f(\bar{x})$ are the proton and anti-proton parton distribution function at momentum fractions $x$ and $\bar{x}$ respectively. The matrix element for the process:

$$
q \bar{q} \rightarrow t t \rightarrow b l^{-} \nu_{l} \bar{b} l^{+} \bar{\nu}_{l} \text {, as wcll as } g g \rightarrow t t \rightarrow b l^{-} \nu_{l} \bar{b} l^{+} \bar{\nu}_{l} .
$$

is denoted by $\mathcal{M}$. Therefore, Equation 8.9 can be expressed by[27]:

$$
p\left(\{o\} \mid m_{t}\right) \propto \int_{\{v\}} f(x) f(\bar{x})|\mathcal{M}|^{2} p(\{o\} \mid\{v\}) d^{18}\{v\} d x d \bar{x}
$$

Evaluation of Equation 8.11 is computationally intense, so we simplify the expression. Later in this chapter we study its implication by comparing the mass of the top quark obtained from this analysis versus the value used for the generation of the top and anti-top quark pair.

The simplificd analytic computation is now described. For every event a weight $\mathcal{W}_{k}$, that corresponds to the $k^{\text {th }}$ ncutrino anti-neutrino momenta solution, and which is a function of the hypothesized mass of the top quark $m_{t}$, is obtained. The method 
is along the same lines as that of Dalitz and Goldstein[72] as well as Kondo[76]. This is established in three steps.

A. At first we map the detected final-state particles in the event to represent particles at the parton level[71]. This involves application of corrections described in the previous chapter, viz. the over-smearing corrections for the electron, muon and the jet 1-momenta, as well as the particle-to-parton level corrections. For the two charged lepton and jet pair, the weight $\left(\mathcal{W}_{k}\right)$ described in the reference [72] is given by:

$$
\mathcal{W}_{k}=\mathcal{W}_{k}\left(\{o\}, m_{t}\right) \propto f(x) f(\bar{x}) p\left(E^{\prime} \mid m_{t}\right) p\left(E^{\prime} \mid m_{t}\right)
$$

This weight represents the probability to measure the set of observables $\{o\}$ using a hypothesized $m_{t}$ and corresponding to the $k^{\text {th }}$ neutrino and anti-ncutrino solution pair. It incorporates the parton distribution function for the proton and anti-proton, and also the decay distribution of the $W$ bosons. In the Equation $8.12 f(x)$ is the proton's parton distribution function evaluated at $Q^{2} \approx m_{t}^{2}$, and $f(\bar{x})$ is the corresponding anti-proton parton distribution function. The expression $p\left(E^{\prime} \mid m_{t}\right)$ is the probability density function for the energy of the charged lepton to be $E^{\prime}$ in the rest frame of the top quark with mass $m_{t}$. This can be analytically represented $\operatorname{as}[77]:$

$$
p\left(E^{\prime} \mid m_{t}\right)=4 m_{t} E^{\prime} \frac{m_{t}^{2}-m_{b}^{2}-2 m_{t} E^{\prime}}{\left(m_{t}^{2}-m_{b}^{2}\right)^{2}+m_{W}^{2}\left(m_{t}^{2}+m_{b}^{2}\right)-2 m_{W}^{4}}
$$

Likewise, $p\left(F^{\prime} \mid m_{t}\right)$ is the probability density function of the anti-lepton energy to be $\bar{E}^{\prime}$ in the anti-top rest frame.

B. Combining all the $n$ solutions for the neutrino momenta, the total event weight 
(W) obtained is cxpressed as:

$$
\mathbf{W}=\mathbf{W}\left(\{0\}, m_{t}\right)=(\text { normalization }) \sum_{k=1}^{n} \mathcal{W}_{k}
$$

C. To account for the detector resolutions, we average the wcight function $\mathcal{W}_{k}$ over the experimental resolutions as well. These object resolutions were listed in Chapter 7 in Tables 7.11, 7.12 and 7.13. Given the measured final-state observables and the hypothesized $m_{t}$, the cvent weight $\mathcal{W}_{k}\left(m_{t}\right)$ represents the likclihood that the event is observed using a hypothesized value of the mass of the top quark. The neutrino and anti-neutrino solutions which are not physical are neglected. A null value of $\mathcal{W}_{k}$ is assigned such that when we sum over all such weights, their contribution is void. Therefore, using Equation 8.14, we can approximate Equation 8.11 as:

$$
p\left(\{o\} \mid m_{t}\right) \approx \mathbf{W}\left(\{o\}, m_{t}\right)
$$

$\Lambda$ distribution of weights $\mathbf{W}$ from every event is used to extract the $m_{t}$ which is consistent with the set of measured kinematic observables from all selected events. The value of the parameter $m_{t}$ which corresponds to the maximum of the distribution is also obtained. The statistical analysis tool used for this purpose is introduced in the noxi scotion.

\subsection{The mass analysis: the first step}

Consider a variable $X$ (which takes values from a set $X^{\prime}$ ) that may be derived from experimental observable(s). Suppose the distribution of this variable is expressed as a function of the unknown parameter, e.g. $m_{t}$ (which may be described by a set 
of values $M$ ). Let the density function of $X$ at $x$ be given by the analytic function $f\left(x \mid m_{t}\right)$. The likelihood function $L$ is the function obtained by exchanging the roles of $x$ and $m_{l}$, so that the parameter to be determined $m_{l}$ is treated as an unknown variable, and the quantity $x$ is treated as the known variable:

$$
L\left(m_{t} \mid x\right)=f\left(x \mid m_{t}\right) \text {, for } m_{t} \text { in } M \text { and } x \text { in } X^{\prime} .
$$

In the method of maximum likelihood, the aim is to establish a value $\mathcal{M}(x)$ of the parameter $m_{t}$ that maximizes $L\left(m_{t} \mid x\right)$ for every $x$ in $X^{\prime}$. The value $\mathcal{M}(x)$ is called the maximum likelihood estimator of $m_{l}$. A choice of this estimator is cxplaincd in the next section, while the evaluation of the maximum likelihood function used in this dissertation is explained in sub-section 8.4.1.

\subsubsection{The peak weight as the mass estimator}

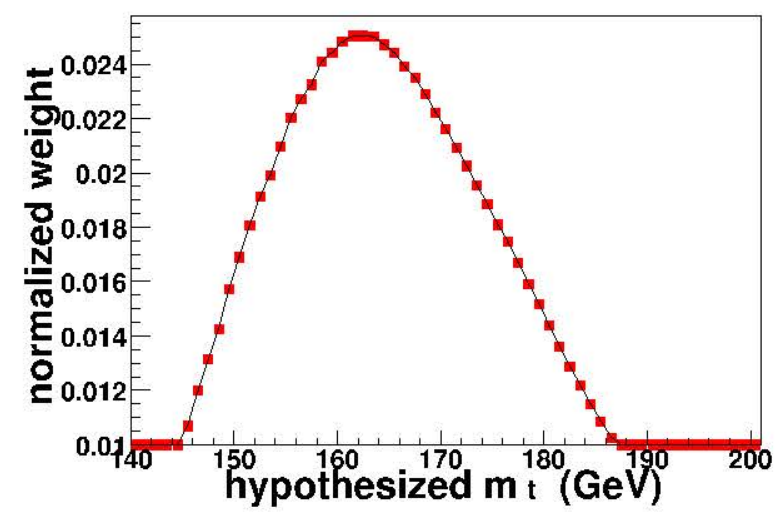

Figure 8.5: The weight distribution of a simulated event generated using $175.0 \mathrm{GeV}$ as the value of the mass of the top quark.

The value of the hypothesized $m_{t}$ corresponding to the global maximum of 
the weight distribution $\mathbf{W}$ represents the best estimate of $m_{t}$ corresponding to the kinematics of that event. As an example, consider Figure 8.5 which illustrates the weight distribution of a simulated event with an input $m_{l}=175.0 \mathrm{GeV}$. From simulated events as well as data events, the peak value $\left(m_{p c a k}\right)$ is used as a mass estimator (which was denoted by $\mathcal{M}$ in the previous sub-section) for the maximum likclihood analysis. This valuc is not an unbiased cstimator of the actual mass[78]. It has been shown[79] that it is a better estimate to the input MC value of $m_{t}$ when compared to the mean of the weight distribution W. In this analysis, hypothesized values of $m_{t}$ with $1.0 \mathrm{GeV}$ increments are used for solving the event kinematics. Therefore, the peak value of the distribution can be off by at most $\Delta \sigma_{\max }^{\text {hypo }}=1.0 \mathrm{GeV}$. This fuctuation is marginal compared to the statistical and systematic uncertainties that are obtained in the measurement.

The $m_{p e a k}$ value determined from an event may not be an ideal representative of the mass of the top quark. However, when we consider these values from many simulated events generated with the same input mass, then the shape of the distribution represents the likelihood of measuring the mass of the top quark as a function of the hypothetical value used to constrain the set of equations mentioned in Section 8.1.1. Analysis of a large number of simulated events shows that kinematic selection cuts used in cvent selcetion introduce a bias in the distribution[81]. Morcover, as cxplained before in Equation 8.15, the wcight function is only an approximation of the probability to measure the event observables $\{0\}$ for a hypothetical value of the mass of the top quark and it is not the exact solution. The presence of effects such as initial state radiation and final state radiation in the event also introduce a bias[78]. IIence, we compare the peak of the weight distribution of events to templates which represent expectations from MC events. This method[83] (using 
template distributions) helps avoid the ellects of unwanted bias in the estimator.

The next section describes the analysis procedure used to extract the most likely value of the mass of the top quark using an ensemble of events.

\subsection{Mass analysis using an ensemble of events}

An un-binned maximum likelihood mothod is an idcal tool for solving this problem, since it works well for ensembles with small number statistics. Application of this method would require an analytical representation of t.emplates from simulated events. Due to the limited availability of simulated events, templates of binned histograms are used to represent likelihood distributions. Finally, a binned maximum likclihood method[85], [89] is used to extract the best estimate of $m_{l}$.

From an ensernble of $N_{\text {tot }}$ selected events we obtain $\left\{m_{\text {peak }}^{1}, m_{\text {peak }}^{2}, \cdots m_{\text {peak }}^{N_{\text {tot }}}\right\}$ mass estimators. Wo assume this distribution follows a probability distribution function $f\left(\left\{m_{\text {peak }}^{i}\right\} \mid m_{t}\right)$ which can be established from template distributions. Details about the construction of template distributions are described in the sub-section 8.4.3. The maximum likelihood function used in the analysis to derive the best estimate of our parameter $m_{t}$, using the estimators $m_{\text {peak }}$ from the ensemble of events is now defincd.

\subsubsection{The Maximum Likelihood Function}

Consider the hypothetical case where we have a set of $N_{\text {tot }}$ entries $\left\{m_{\text {peak }}^{1}, m_{\text {peak }}^{2}\right.$; $\left.\cdots m_{\text {peak }}^{N_{t}}\right\}$ which are binned in $N$ bins (of a histogram). If the entries in cach of the 
bins are $n_{1}, n_{2}, n_{3} \cdots n_{N}$, simply denoted by $\vec{n}$, then

$$
\sum_{i=1}^{N} n_{i}=N_{t o t}
$$

Consider $N_{\text {lol }}$ as a random variable from a Poisson distribution with a mean valuc of $\nu_{\text {tot }}$. We first determine the probability of obtaining $N_{\text {tot }}$ using the Poisson probability distribution function and then distribute the observations of the $m_{\text {peak }}$ in a histogram with $N$ bins, the bin content of which is denoted by $\vec{n}$. The joint probability distribution function for obtaining $N_{t o t}$ with corresponding bin contents given by $\vec{n}$ is:

$$
f_{\text {joint }}(\vec{n} \mid \vec{\nu})=\frac{\nu_{\text {tot }}^{N_{\text {tot }}} e^{-\nu_{\text {tot }}}}{N_{\text {tot }} !} \cdot \frac{N_{\text {tot }} !}{n_{1} ! n_{2} ! \cdots n_{N} !}\left(\frac{\nu_{1}}{\nu_{\text {tot }}}\right)^{n_{1}}\left(\frac{\nu_{2}}{\nu_{\text {tot }}}\right)^{n_{2}} \cdots\left(\frac{\nu_{N}}{\nu_{\text {tot }}}\right)^{n_{N}}
$$

where in the above equation the probability for an entry to be in bin $i$ has been cxpressed as the expectation value $\nu_{i}$ divided by $\nu_{t o t}$, where:

$$
\nu_{t o t}=\sum_{i=1}^{N} \nu_{i}
$$

The Equation 8.18 can be simplified to:

$$
f_{\text {joint }}(\vec{n} \mid \vec{\nu})=\prod_{i=1}^{N} \frac{\nu_{i}^{n_{i}}}{n_{i} !} e^{-\nu_{i}}
$$

The expectation value of the number of entries in the $i^{\text {th }}$ bin $\left(\nu_{i}\right)$ is given by:

$$
\nu_{i}=\nu_{i}\left(\nu_{l o l} \mid m_{t}\right)=\nu_{\text {lot }} \int_{m_{i}^{\text {min }}}^{m_{i}^{\text {max }}} \int\left(\left\{m_{\text {peak }}\right\} \mid m_{i}\right) d m_{\text {peak }}
$$


where $m_{i}^{\min }$ and $m_{i}^{\max }$ are the bin limits. Taking the logarithm of the joint probability distribution function in Equation 8.18 and omitting the terms which do not depend on the mass parameter, the logarithm of the likelihood function for the binned histogram of $m_{p c a k}$ values can be expressed as:

$$
\log L\left(\nu_{t o t} \mid m_{t}\right)=\sum_{i=1}^{N}\left(n_{i} \log \nu_{i}-\nu_{i}\right)=\sum_{i=1}^{N} n_{i} \log \nu_{i}-\nu_{t o t} .
$$

Altcrnatively, if the number of entries in cach of the $i^{\text {th }}$ bin are distributcd randonly, having Poisson probability distribution function with a mean value $\nu_{i}$, then the probability density will also be given by Equation 8.18[85]. Equation 8.22 is the log-likelihood function used for obtaining the maximum likelihood estimate (MLE) of $m_{t}$ from an ensemble of events ${ }^{4}$.

The set of expectation values for entrics

$$
\vec{\nu}=\vec{\nu}\left(m_{t}\right)=\left\{\nu_{1}, \nu_{2} \cdots \nu_{N}\right\}
$$

in the Equation 8.22 is obtained from tcmplate histograms which are constructed from many simulated events. The estimator $m_{\text {peak }}$ from the ensemble of data events is used to construct the ensemble histogram. The entries in those histogram bins correspond to the set of numbers denoted by

$$
\vec{n}=\left\{n_{1}, n_{2} ; \cdots n_{N}\right\}
$$

For performing tests using simulated events, $\vec{n}$ is obtained from the histograms using the lone estimator $m_{\text {peak }}$ from every event. Figure 8.6 illustrates the values

\footnotetext{
${ }^{4}$ In this dissertation, the total number of entries in the ensemble histogram is kept fixed, while
} 


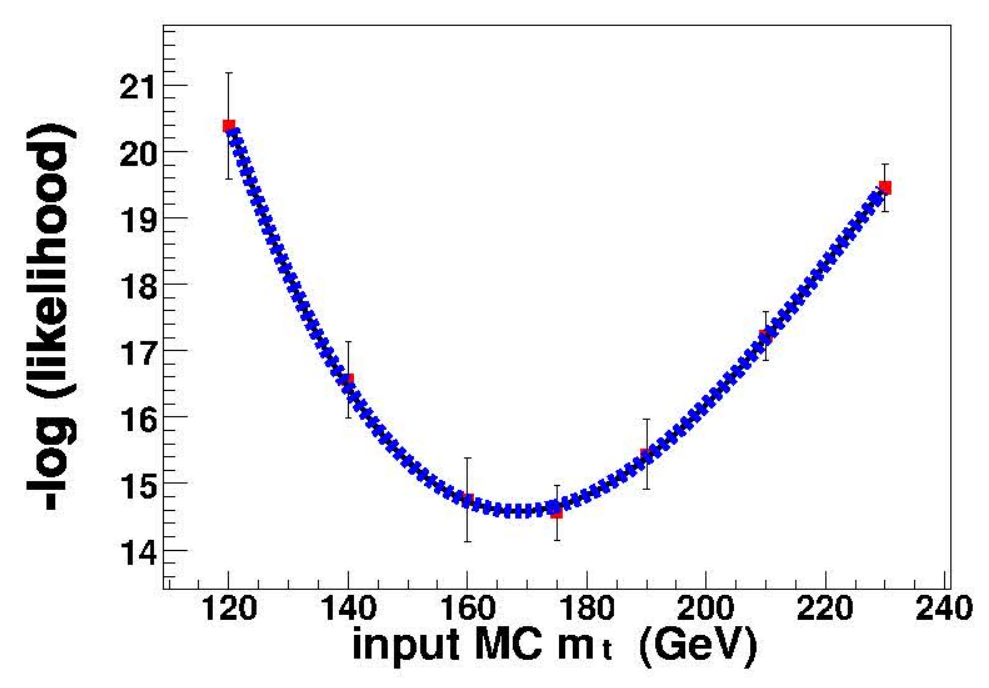

Figure 8.6: An example of the maximum log likelihood fit. The minimum from the numerical fit is minimum of the likelihood function and it corresponds to the most likely estimate (MIF) of the parameter (the mass of the top quark) for the ensemble of events.

of the negative of the log-likclihood distribution using Equation 8.22 as a function of the parameter $m_{t}$ for an ensemble of simulated events. The template histograms used in the study are shown in Figure 8.8 and 8.9. The best estimate for the simulated ensemble corresponds to the minimum of the (best) numerically generated fit obtained from the distribution of the negative logarithm of the likelihood versus the input MC $m_{t}$. The best fit to the set of points is obtained using the numerical analysis package MINLIT in ROOT[84].

For likelihood functions $L$ which are Gaussian distributions, maximum loglikelihood function correspond to curves which are quadratic in nature[89]. However, the template histograms are not Gaussian distributions. Therefore, an asymmetric function is used to fit over the range of $m_{t}$. The the rrost likely estimate of the the number of entries in each of the individual bins are randomly distributed. 
mass of the top quark corresponds to the minimum of the best mumerical fit to the likelihood distribution. The simplest asymmetric fit (a cubic function) is used for this analysis. Compared to the quadratic functional form used in the numerical fit, the cubic fit is a better fit in most cases. 


\subsubsection{Statistical uncertainty from ensemble studies}

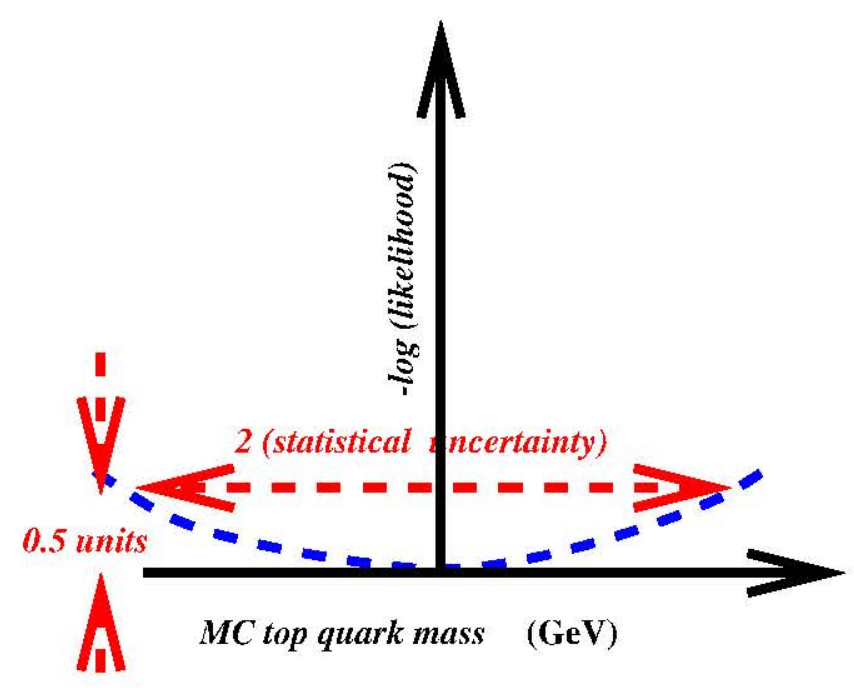

Figure 8.7: A schematic illustrating the evaluation of statistical uncertainty in the evaluation of the maximum likelihood estimate (MLE) from an coscmble of events. The dotted (parabolic curve) represents the best numerically obtained fit around the neighborhood of the global minimum.

Using Gaussian tcmplates and enscmble histograms which are Gaussian distributions, the standard procedure[89] of establishing the statistical uncertainty is illustrated below.

From the numerical fit illustrated in Figure 8.6 and Figure 8.7, let the global minimum (MLE) of the $x$-axis be $\left(x_{\text {min }}\right)$. Let its corresponding log-likelihood value be denoted by $y_{\min }$ (say). The statistical uncertainty $\sigma_{\text {slat }}$ (within a $\sim 68 \%$ confidence limit) for the determined MLF corresponds to the values of $m_{t}$ which are within $y_{\min } \pm 0.5$. If this stratcoy is repcated for many ensembles, and a distribution of the pull ${ }^{5}$ from all ensembles is a Gaussian distribution, with a mean of zero and unit RMS. The mean value of zero reflects the fact that there is a null bias in de-

\footnotetext{
${ }^{5}$ The pull from every ensemble is defined as (fitted $m_{t}-$ input MC $\left.m_{t}\right) /\left(\sigma_{\text {stat }}\right.$ )
} 
termining the MLE. The unit value of the RMS of the Gaussian distribution shows that the statistical uncertainty is estimated within a $\sim 68 \%$ confidence limit.

The same idea is used in this analysis. However, instead of estimating the statistical fluctuation at only $y_{\min } \pm 0 . \bar{j}$, we evaluate it at $y_{\min } \pm(0.5+N \times 0.075)$; where $(N=1,2,3)$. The pull distribution corresponding to all these cases are determined. It is observed that when the limits are determined at $y_{\min } \pm 0 . \overline{0}$., the pull distributions better represent unit Gaussian distributions. 


\subsubsection{Template construction}

Figure 8.8 and Figure 8.9 represent the template distributions used for the analysis in the e $\mu$ channel. Similar plots of the template distributions for the di-electron channel are illustrated in Figure 8.10. From studies done previously (which may be found in [27] and [80]), estimates were made for establishing the statistical un-

certainty $\left(\Delta \sigma_{\text {stat }}\right)$ associated with the measurement of the mass using simulated cnscmbles having small number statistics ( $\sim 10$ cvents). The algorithm used in this thesis yields $\Delta \sigma_{\text {stat }} \approx 16$ to $19 \mathrm{GeV}[81]$ for ensembles of eight events with $\approx 20 \%$ background contamination. It is not possible to gencrate MC cvents with a continuously varying input $m_{t}$, nor is it feasible to generate them for a wide range of hypothetical $m_{t}$. The samples are generated over a range of hypothetical $m_{t}$ values, spanning about three to four times the $\Delta \sigma_{\text {stat }}$ from the assumed central value of 175.0 GeV. This helps avoid bias which may occur at lower or higher ends of the fitted mass range, while numerically extracting the maximum likelihood fit. The input $m_{t}$ values used to generate the signal MC template distributions are 120.0, $140.0,160.0,175.0,190.0,210.0$, and $230.0 \mathrm{GeV}$.

Primarily two types of templates are used in this study. For studies with signal ensembles templates from the three di-lepton signal processes are used. Templates representing contamination from background processes are also constructed and then added to signal templates. A random multinomial admixture of events from signal and background processes are used. The sources of background contamination and their average proportion in an ensemble are illustrated in Table 8.1[52].

If many thousands of unique simulated events are used to construct template histograms, then the histograns can be binned in small intervals, e.g., $5 \mathrm{GeV}$, and the bin-to-bin fluctuations in those templates would be minimal. In this analysis 
statistics obtained for template construction is limited, and the results obtained for the most likely value of the ensembles depend on the nature of template construction. This prompts us to check the sell-consistency of the algorithm used. The selfconsistency tests using simulated ensembles are discussed in Section 8.5. It has been empirically established[87] that the optimal histogram bin size, which provides an unbiased estimation of the probability density represcnted by a histogram is achieved for:

$$
\Delta w=3.5 \sigma N^{-\frac{1}{3}}
$$

where $\Delta w$ is the width of the histogram bin, $\sigma$ is the standard deviation of the distribution and $N$ is the statistics available. Similar results have been obtained by Freedman and Diaconis[88]. They establish a bin width given by:

$$
\Delta w=2(I Q R) N^{-\frac{1}{3}}
$$

where $I Q R$ is the inter-quartile range (the $75^{\text {th }}$ percentile minus the $25^{\text {th }}$ percentile). In both formulations the width is proportional to $N^{-\frac{1}{3}}$.

For this analysis, template binning of $30 \mathrm{GeV}$ is used. A summary of event criteria and relevant details regarding the template statisties is described in Appendix G. For the studies done with a simple-minded Monte Carlo (Pythia[44], without detector resolution effects) binning from values of $20 \mathrm{GeV}$ upto even $10 \mathrm{GeV}$ was used. In this casc the statistics for signal processes were nearly twenty times as much as what was available from the complete D $\varnothing$ detector simulated Monte Carlo events. The statistics for background processes used were of the same order as those from signal processes. 

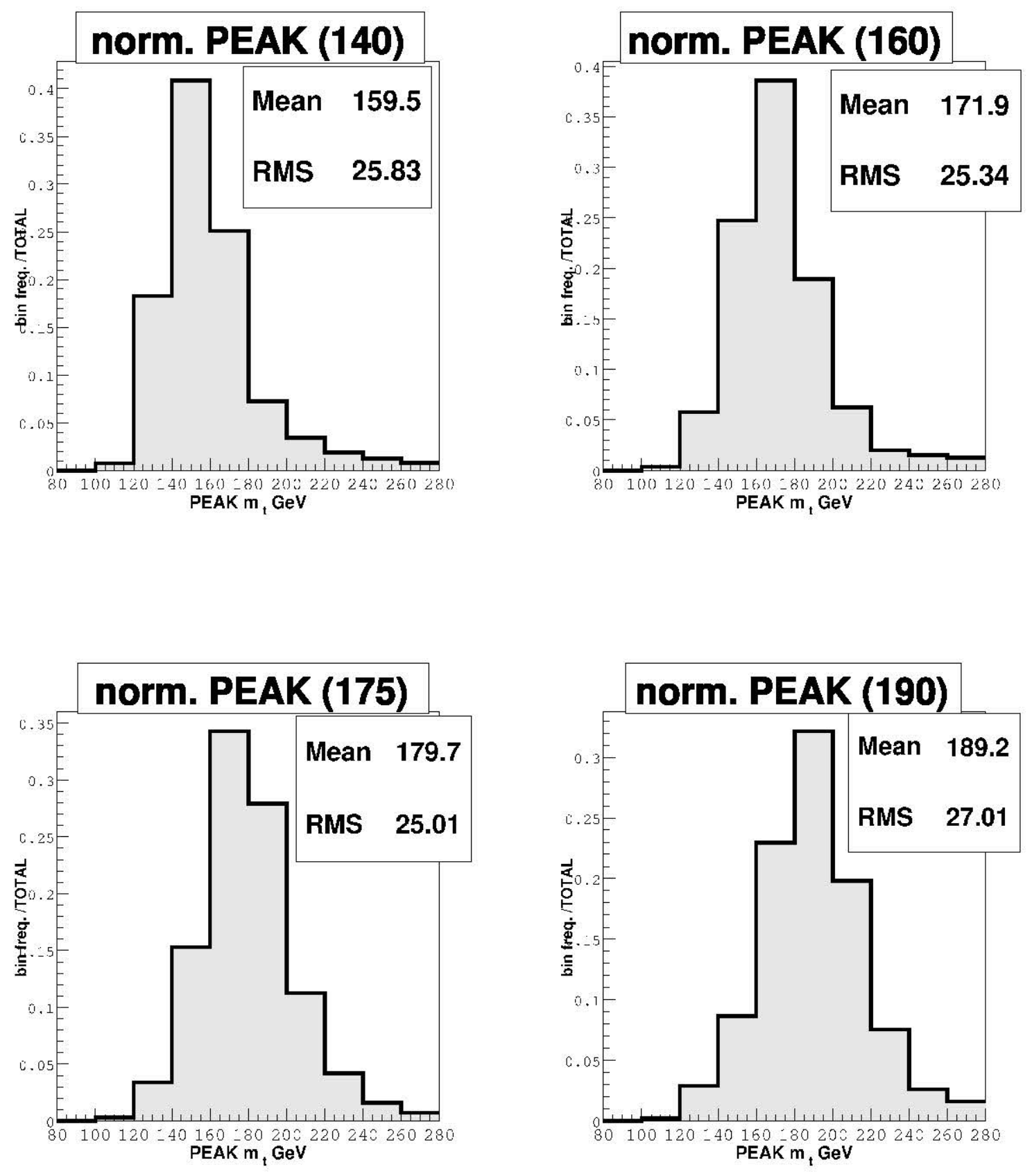

Figure 8.8: Template distributions for analysis in the $e \mu$ channel. 

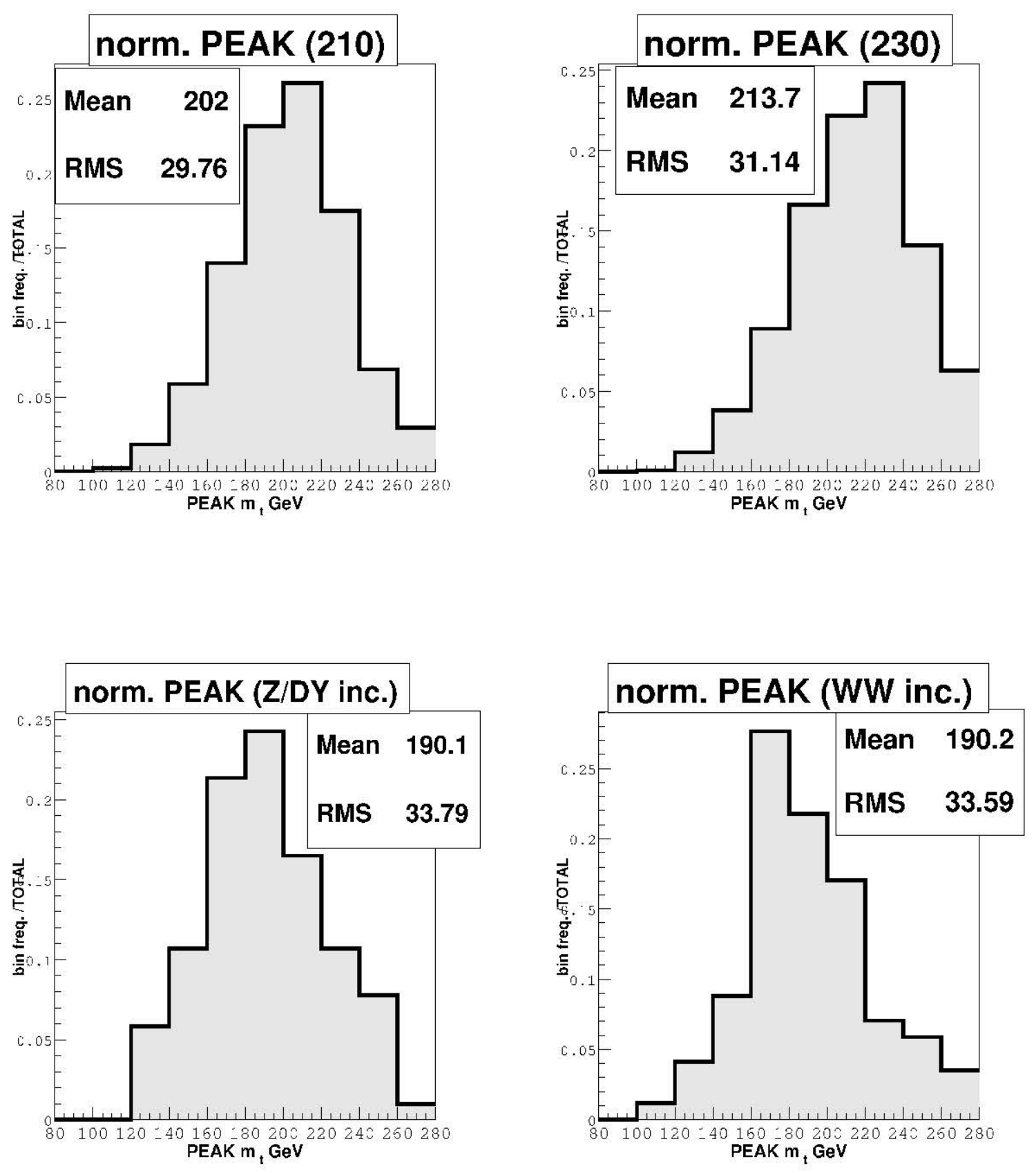

Figure 8.9: More template distributions for analysis in the $e \mu$ channel. 

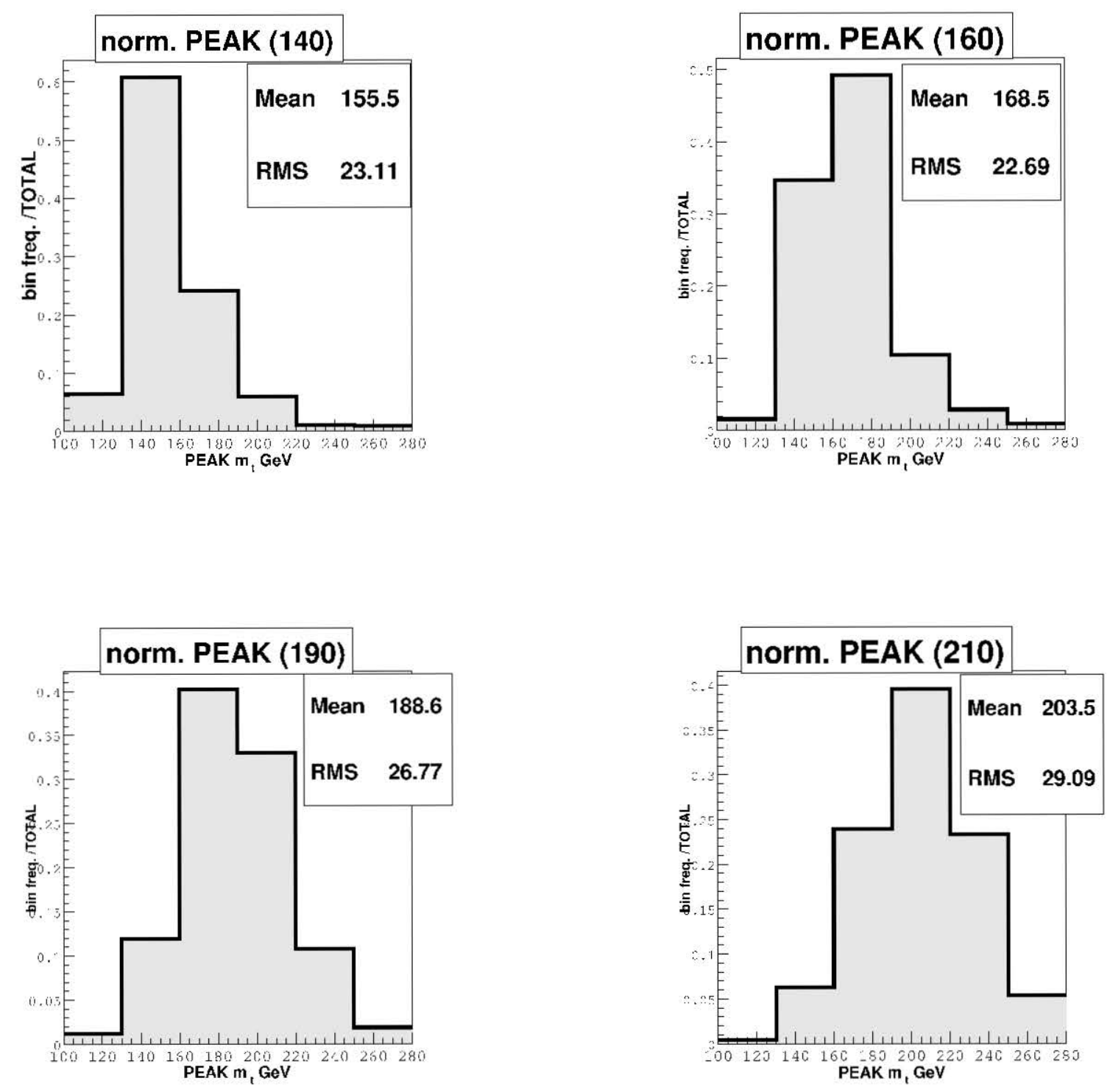

Figure 8.10: Some template distributions for analysis in the ee channel. 


\subsubsection{The data ensemble}

The Figure 8.11 and Figure 8.12 are the data histograms of $m_{\text {peak }}$ values from the selected data events in the $e \mu$ channel and the di-electron channel respectively. Events obtained after application of each and every selection criterion described in Chapter 6 as well as in [52] constitute the ensemble of data events. The number of events obtained in each channel is given in Table 8.1.

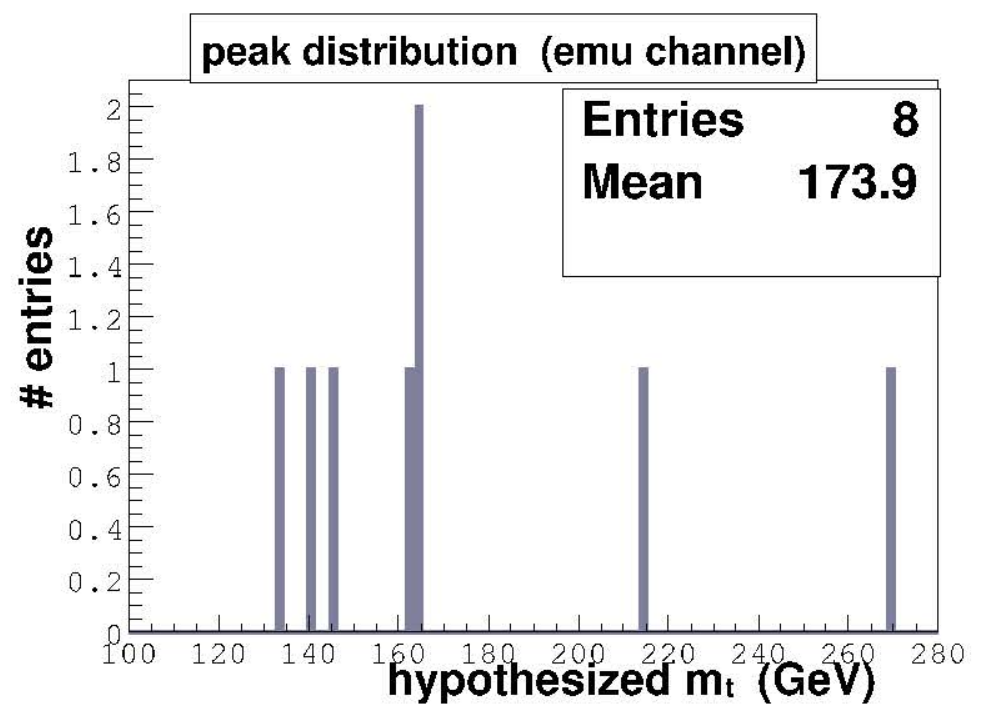

Figure 8.11: ITistogram of the $m_{\text {peak }}$ distribution from the selected data events in the e $\mu$ channel.

The values of $m_{\text {peak }}$ from the weight distributions of each of the selected events are populated in histograms whose bin widths are identical to those of the template histograms ${ }^{6}$.

\footnotetext{
${ }^{6}$ The bin width in the histograms in Figures 8.11 and 8.11 is $\sim 2 \mathrm{GeV}$.
} 


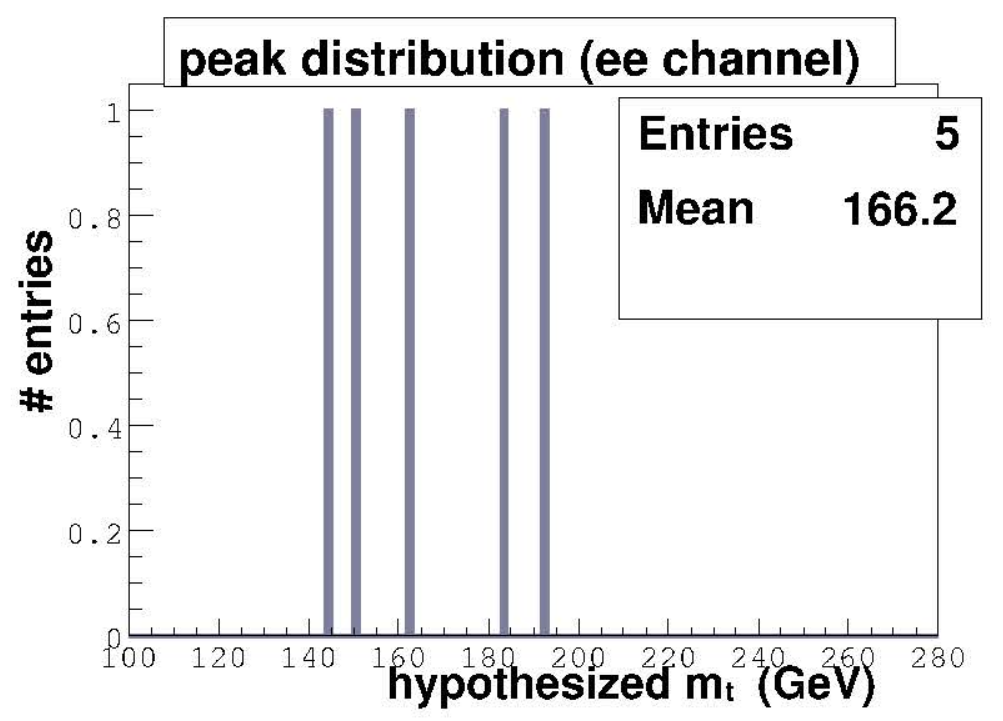

Figure 8.12: Histogram of the $m_{\text {peak }}$ distribution from the selected data events in the di-clectron channel.

\subsubsection{Construction of simulated ensembles for self-consistency tests}

Simulated events are also filtered using kinematic and quality critcria identical to those used in selecting data events[52]. As explained before, the peak values $\left(m_{\text {peak }}\right)$ from the event weight of the simulated events corresponding to an ensemble are binned into an ensemble histogram. All available simulated cvents are used to construct independent ensemble histograms. The number of events used to construct such simulated ensembles is set to the number of events observed in each of the di-lepton channels. The main ingredients of ensemble composition are listed in Table 8.1. 


\begin{tabular}{|c|c|c|c|}
\hline & $\mathrm{e} \mu$ & ee & $\mu \mu$ \\
\hline & channel & channel & channel \\
\hline integrated luminosity $\mathrm{pb}^{-1}$ & 228.29 & 213.00 & 221.33 \\
\hline \# candidate events & 8 & 5 & 0 \\
\hline$\%$ signal contribution & 84.46 & 67.25 & 53.08 \\
\hline$\% Z^{0} / \gamma^{*}$ inclusive processes & 6.47 & 4.59 & 39.04 \\
\hline$\% W^{+} W^{-}$inclusive processes & 5.83 & 4.93 & 5.48 \\
\hline$\%$ instrumental cffects & 3.21 & 23.23 & 2.40 \\
\hline$\%$ total background contribution & 15.54 & 32.75 & 16.92 \\
\hline
\end{tabular}

Table 8.1: The composition of templates and simulated ensembles expressed as a percentage of the total composition. The information from this Table is obtained from $[52]$.

\subsection{Evaluation of the Maximum Likelihood Estimate}

The Maximum Likelihood Estimate (MLE) from an ensemble corresponds to the numerically obtained minimum of the log-likelihood fit such as the one in Figure 8.6. This is the most likely estimate of the value of the mass of the top quark obtained using the $m_{\text {peak }}$ estimators from the cnscmble of events.

We can use the MIFs obtained from simulated ensembles to determine the contribution of systematic uncertaintics.

\subsubsection{The Maximum Likelihood Estimate using simulated ensembles}

Figure 8.13 represents the distribution of the maximum likelihood estimates obtained from 100 independent ensembles which have $80 \%$ signal events generated with input $m_{t}=175.0 \mathrm{GcV}$ and $20 \%$ background contribution in them. The distribution 
can be fitted to a Gaussian form. We use the mean of the distribution as the most likely estimate of the generated or input $m_{t}$. It is well established that for a Gaussian distribution, the mean is the maximum likelihood estimator[89]. Therefore we numerically fit the MLF distribution to a Gaussian function and use the mean and variance from the numerical fit for the estimated value of $m_{t}$ and its statistical uncertainty respectively. Although the variance of the Gaussian distribution is biased, it is possible to correct for that bias.

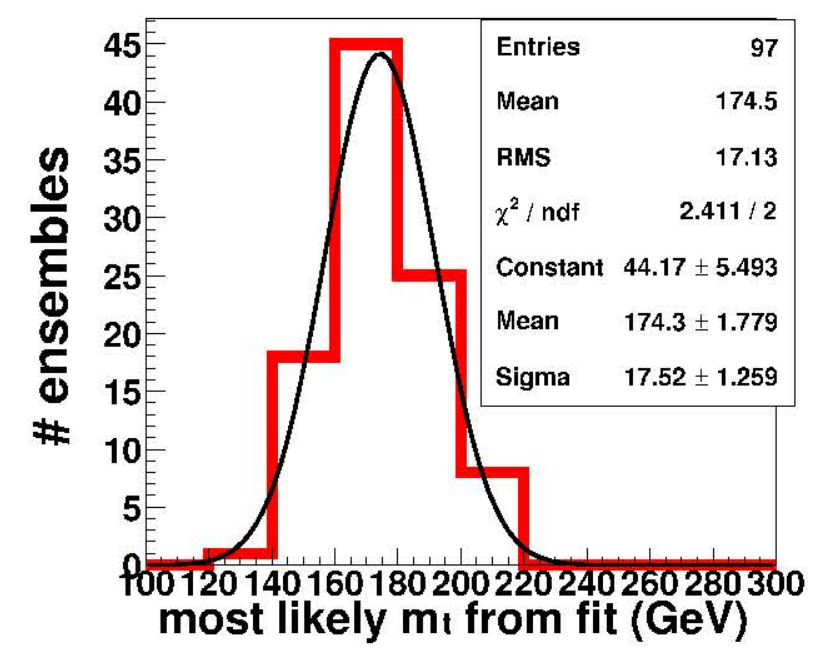

Figure 8.13: Histogram of the most likely values from 100 simulated ensembles each with eight events.

Starting with various input values of the generated mass of the top quark, similar studies were done. Table 8.4 shows the expected statistical uncertainty from tests using simulated $e \mu$ ensembles of 8 events. Results from a similar case study with simulated di-electron event ensembles ( 5 event per ensemble) are presented in Table 8.5. The set of the MLE distribution and pull distributions obtained in these studies are illustrated in Appendix I. 


\begin{tabular}{|r|r|r|r|r|r|}
\hline input $\mathbf{M C} m_{t}(\mathrm{GeV})$ & $\mathbf{1 4 0}$ & $\mathbf{1 6 0}$ & $\mathbf{1 7 5}$ & $\mathbf{1 9 0}$ & $\mathbf{2 1 0}$ \\
\hline \# ensembles & 241 & 239 & 194 & 294 & 289 \\
\hline fitted $m_{l}(\mathrm{GcV})$ & 140.5 & 160.7 & 176.3 & 192.1 & 210.0 \\
\hline RMS of mean $(\mathrm{GeV})$ & 17.26 & 19.24 & 18.60 & 20.23 & 17.39 \\
\hline pull $(\mathrm{GeV})$ & -0.008 & -0.04 & -0.03 & +0.15 & +0.07 \\
\hline RMS of pull $(\mathrm{GeV})$ & 0.82 & 1.08 & 1.02 & 1.07 & 0.90 \\
\hline
\end{tabular}

Table 8.2: Results from simulated ensembles of 8 e $\mu$ events using 140, 160, 175, 190, and $210 \mathrm{GeV}$ as the input $m_{t}$.

\begin{tabular}{|r|r|r|r|r|r|}
\hline input $\mathbf{M C} m_{t}(\mathrm{GeV})$ & $\mathbf{1 4 0}$ & $\mathbf{1 6 0}$ & $\mathbf{1 7 5}$ & $\mathbf{1 9 0}$ & $\mathbf{2 1 0}$ \\
\hline \# cnscmbles & 128 & 142 & 160 & 159 & 143 \\
\hline fitted $m_{l}(\mathrm{GeV})$ & 143.7 & 164.2 & 178.8 & 186.3 & 208.7 \\
\hline RMS of mean $(\mathrm{GeV})$ & 23.08 & 22.03 & 25.18 & 22.63 & 26.48 \\
\hline pull $(\mathrm{GeV})$ & 0.07 & 0.12 & +0.12 & -0.09 & 0.86 \\
\hline RMS of pull $(\mathrm{GeV})$ & 0.86 & 0.95 & 0.91 & 0.89 & 1.04 \\
\hline
\end{tabular}

Table 8.3: Results from sinulated ensembles of 5 di-electron events using 140, 160 , 175,190 , and $210 \mathrm{GeV}$ as the input $m_{t}$. 


\subsection{Self-consistency tests using fast MC}

The following consistency tests are primarily done to check for bias due to oversight or bugs in the kinematic likelihood fitting, or other unforeseen problems.

When an analysis similar to that in Section 8.5 is undertaken for 100 simulated ensembles, signal events having an input $m_{t}$ of $175.0 \mathrm{GeV}$, then we obtain the mcan fitted mass of $174.5 \mathrm{GcV}$, while nearly $3 \%$ of the cnscmbles yicld un-physical solutions. The contribution of simulated background-like events are multinomially incorporated into cnsembles. We repeat the experinents many nore times, with predetermined fraction of signal and constituent background processes. In this way a more appropriate representative of the mean fitted value of $m_{t}$ is obtained. This mean value obtained by the algorithm is used as the measured mass of the top quark, for simulated signal events with input $m_{t}$ value of $175.0 \mathrm{GeV}$.

The above procedure is repeated for signal cvents generated with various other mass points[90]. The set of points obtained can then be used to construct a calibration curve of the average value of the maximum likelihood estimates versus the value of the mass of the top quark used in their generation. The best numerical fit to the set of points is shown in Figure 8.14. If the analysis algorithm is perfect then the best fit to the set of independent measurements would correspond to a straight line with unit slope and an offset corresponding to the nominal value of $175.0 \mathrm{GeV}$. The tests were done using many (500) events per ensemble, to avoid any effects due to small statistics that may creep in and produce a bias. In the first case (results shown on the top plot in Figure 8.14) the ensembles were derived from events which went in to constitute the template distributions. $\Lambda$ straight line parameterized as:

$$
\text { fitted mass }=p 1 \cdot(\text { input mass }-175.0 \mathrm{GcV})+p 0 \mathrm{GcV}
$$


gives the best fit to the cnscmble test results for $p 1=1.007 \pm 0.09$, and $p 0=175.7$ $\pm 0.2 \mathrm{GeV}$. This fit to the set of points shown in Figure 8.14 is consistent with a straight line of unit slope and a nominal offset of approximately $175 \mathrm{GcV}$.

The results illustrated in the bottom plot in Figure 8.14 are from an independent test. In this test, it was ensured that the events which were used for ensemble construction were not used for template distributions, but other cvents corresponding to the relevant signal and background processes were used.

The calibration curves shown in Figure 8.15 are obtained using ensembles with small number statistics. The top plot is obtained using ensembles with 8 events, while the bottom plot in Figure 8.15 is obtained using 5 events per ensemble. The background contamination in both studies were kept the same, nearly $15 \%$. The best straight line fits in these independent tests correspond to a straight line of unit slope and a nominal offset of $175.0 \mathrm{GcV}$. This is a proof that the analysis algorithm is self-consistent.

These results rellect the lact that the developed dynamical likelihood fitting method is self-consistent. If there are any biases, then they are at a level much smaller than that due to fluctuations in the calibration curve for the case of ensembles with small event statistics (plots in Figure 8.15). 

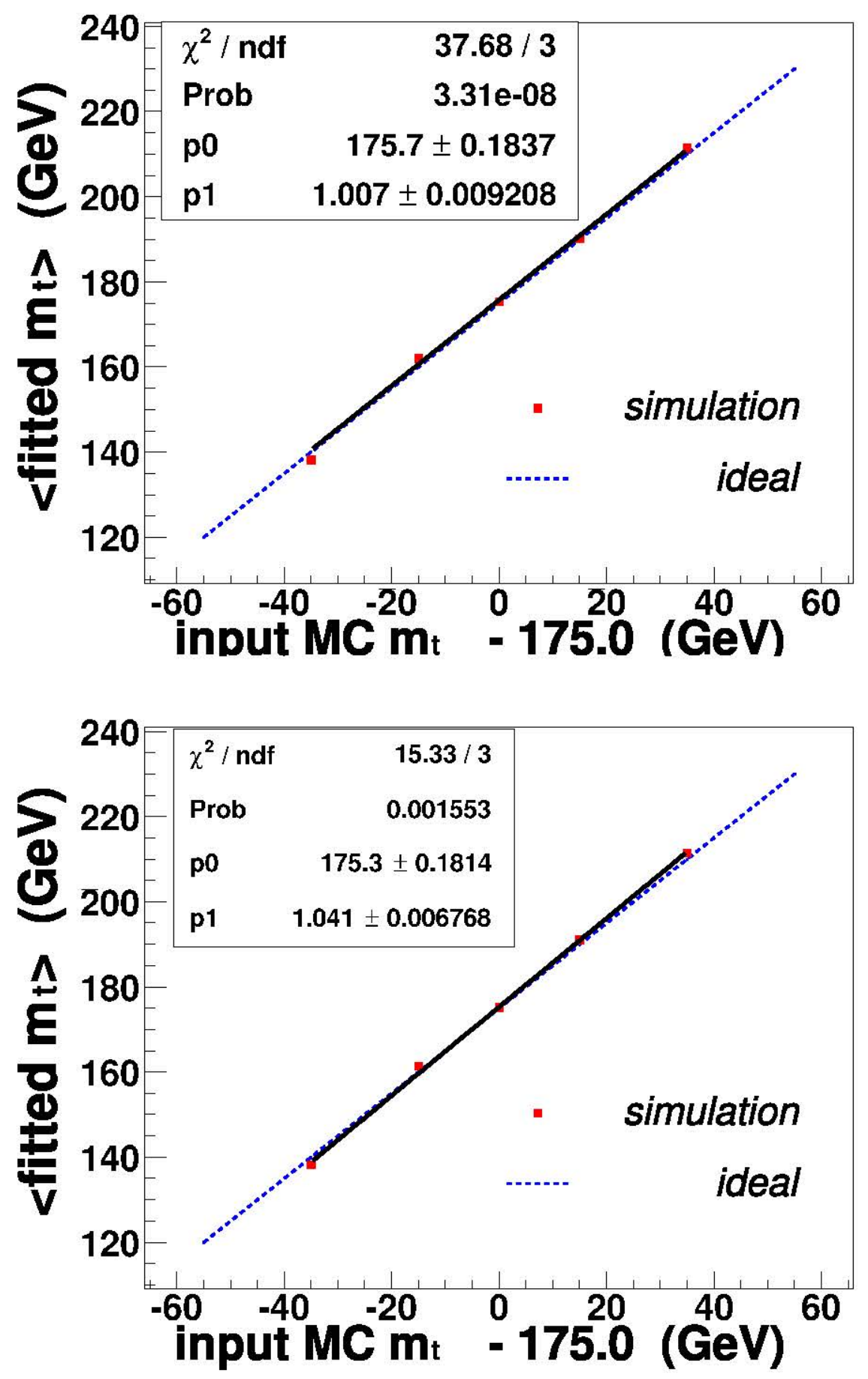

Figure 8.14: Calibration curve from simulated ensembles constructed using signal and background events from Pythia[44]. The bottom plot represents the calibration curve when the events used in constructing templates and simulated ensembles were separated. This was not ensured for the calibration curve displayed on the upper plot. 

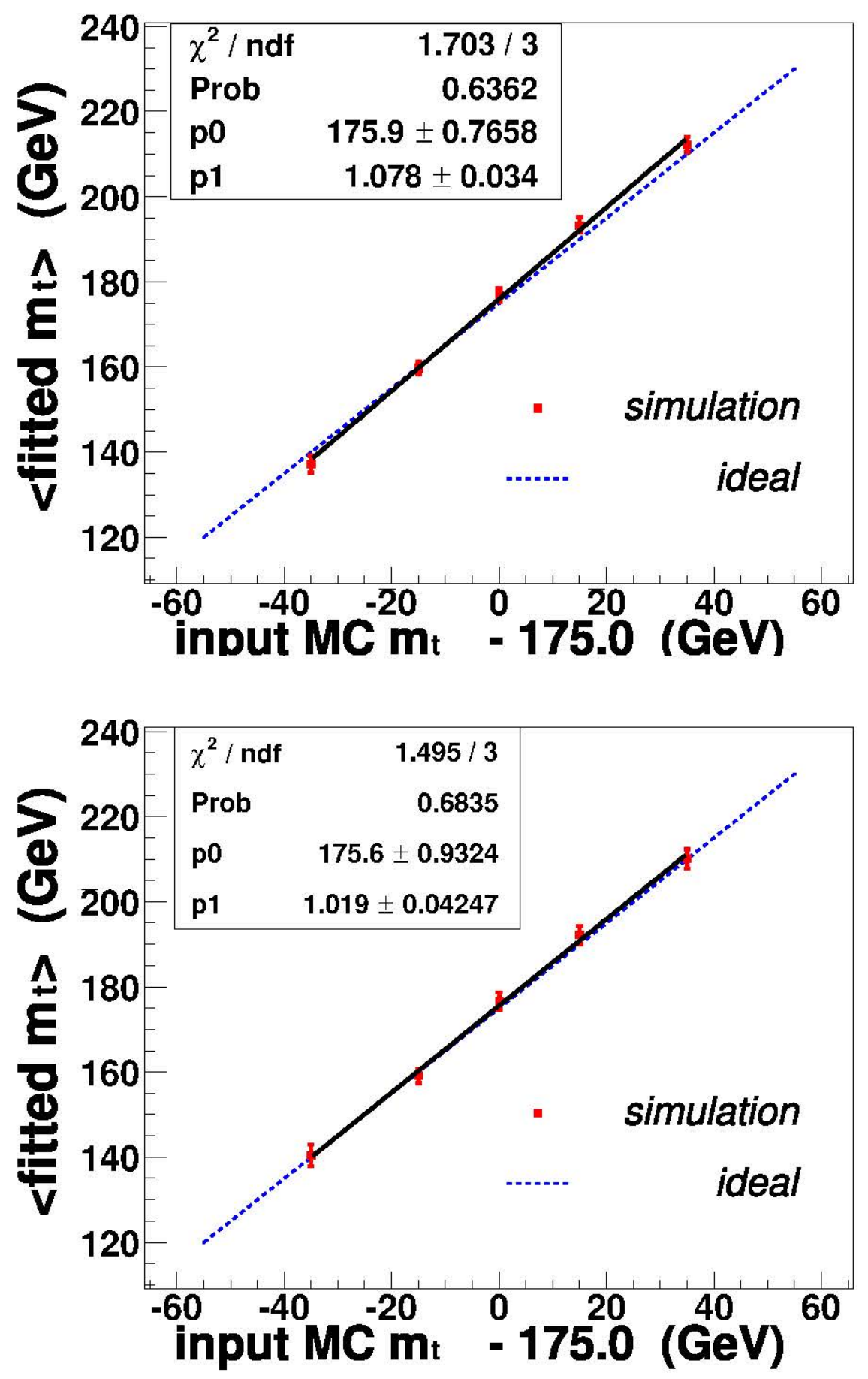

Figure 8.15: Calibration curve from simulated ensembles constructed using signal and background events from Pythia[44]. The upper plot represents the calibration curve obtained using 8 signal and background (15\%) events per ensemble, while the bottom plot represents the calibration curve when only 5 events were per ensemble. 


\subsection{Self-consistency tests with simulated events incorporating the full detector resolution ef-} fects

\begin{tabular}{|c|c|c|c|c|c|}
\hline input $\mathrm{MC} \mathbf{m}_{\mathbf{t}}(\mathrm{GeV})$ & 140 & 160 & 175 & 190 & 210 \\
\hline fitted $<m_{t}>(\mathrm{GeV})$ & 139.89 & 159.60 & 176.91 & 192.06 & 209.06 \\
\hline$<R M S$ of mean $>(\mathrm{GeV})$ & 18.71 & 17.53 & 17.62 & 20.61 & 18.27 \\
\hline$<\frac{R M S}{\sqrt{N_{r i x p l}}}>(\mathrm{GeV})$ & 1.92 & 1.78 & 1.77 & 2.08 & 1.84 \\
\hline$<$ pull $>(\mathrm{GeV})$ & -0.05 & -0.07 & -0.02 & +0.06 & +0.09 \\
\hline$<R M S$ of pull $>(\mathrm{GeV})$ & 0.91 & 0.92 & 0.96 & 1.11 & 0.92 \\
\hline
\end{tabular}

Table 8.4: Results from simulated ensembles with 8 e $\mu$ events using 140, 160, 175 , 190 , and $210 \mathrm{GeV}$ as the input $m_{t}$. Fvery result is the mean of 160 independent random ensembles, which are re-shuffled and used 25 times. The average the number of unique ensembles used $\left(N_{\text {expt }}\right)$ is 100 .

\begin{tabular}{|c|c|c|c|c|c|}
\hline input $\mathrm{MC} \mathbf{m}_{\mathbf{t}}(\mathrm{GeV})$ & 140 & 160 & $\mathbf{1 7 5}$ & 190 & 210 \\
\hline filted $<m_{l}>(\mathrm{GoV})$ & 112.95 & 163.76 & 178.46 & 190.81 & 209.50 \\
\hline$<R M S$ of mean $>(\mathrm{GeV})$ & 24.01 & 23.62 & 23.85 & 24.43 & 25.82 \\
\hline$<\frac{R M S}{\sqrt{N_{\operatorname{expt.}}}}>(\mathrm{GcV})$ & 3.14 & 3.05 & 3.06 & 3.15 & 3.32 \\
\hline$<$ pull $>(\mathrm{GeV})$ & -0.10 & -0.08 & 0.00 & +0.03 & +0.10 \\
\hline$<$ RMS of pull $>(\mathrm{GeV})$ & 0.93 & 1.01 & 0.93 & 0.97 & 1.01 \\
\hline
\end{tabular}

Table 8.5: Results from simulated cnscmbles with 5 di-clectron cvents using 140, 160 , 175,190 , and $210 \mathrm{GeV}$ as the input $m_{t}$. Every result is the mean of 160 independent random ensembles, which are re-shuffled and used 25 times altogether. The number of unique set of ensembles $\left(N_{\text {expt }}\right)$ is 64 .

All the steps described previously are repeated using the simulated events with the full detector resolution effects. Figure 8.16 describes the calibration obtained in the e $\mu$ channel. The relevant information is listed in Table 8.4. Calibration for the analysis in the di-clectron channel is illustrated in Figure 8.18, and Table 8.5 lists 
the relevant statistic.

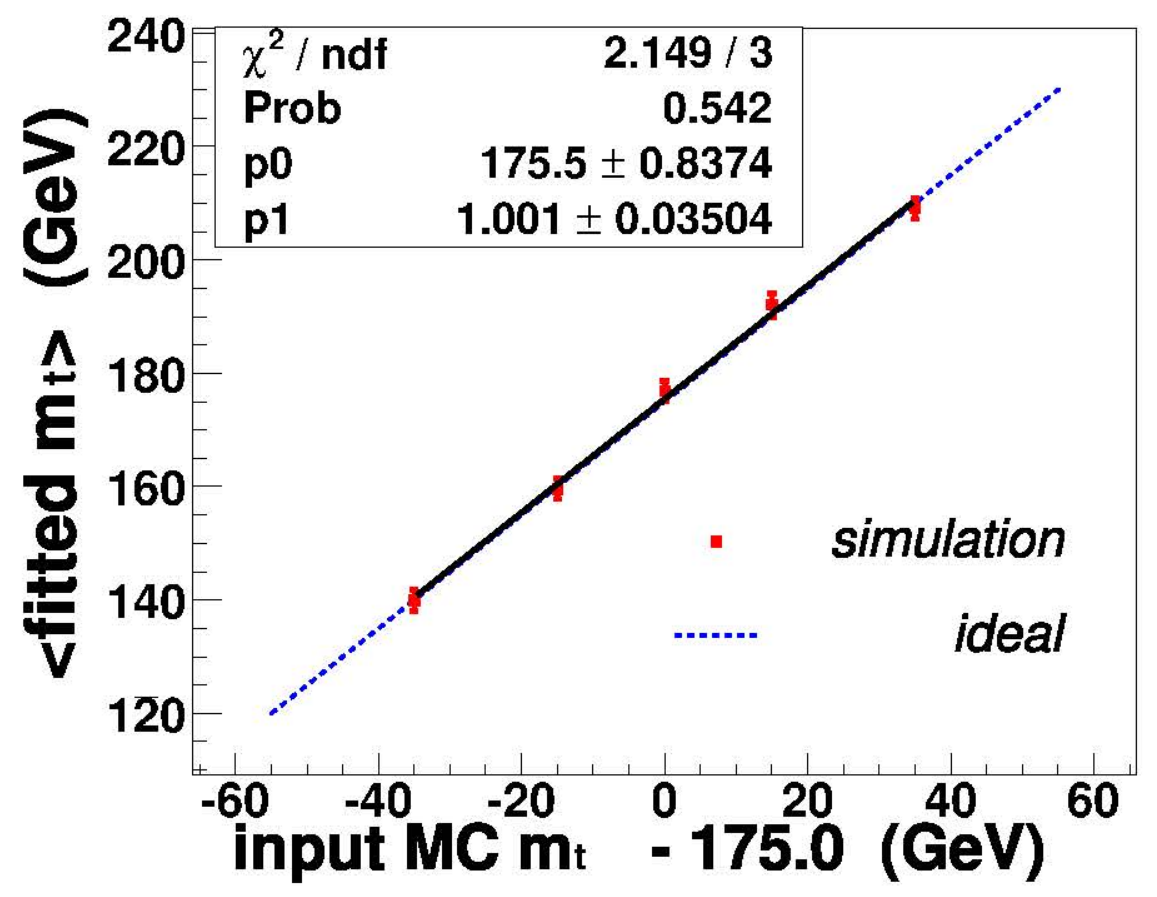

Figure 8.16: Calibration curve from simulated ensembles constructed using signal and background processes of the $e \mu$ channel.

As shown previously, the straight line parameterized as:

$$
\text { average fitted mass }=p 1 \cdot(\text { input mass }-175.0 \mathrm{GeV})+p 0 \mathrm{GeV}
$$

gives the best fit to the cnscmble test results for $p 1=1.001 \pm 0.03$, and $p 0=175.5$ $\pm 0.8 \mathrm{GeV}$. This fit to the set of points shown in Figure 8.16 is consistent with a straight line of unit slope and the nominal oflset of $175 \mathrm{GeV}$.

It has been shown (in Appendix J) that when the purity of the ensembles is reduced, then the fitted parameters are less likely to be consistent with a straight line of unit slope and an offset of $175.0 \mathrm{GeV}$ than that with lesser or no background 


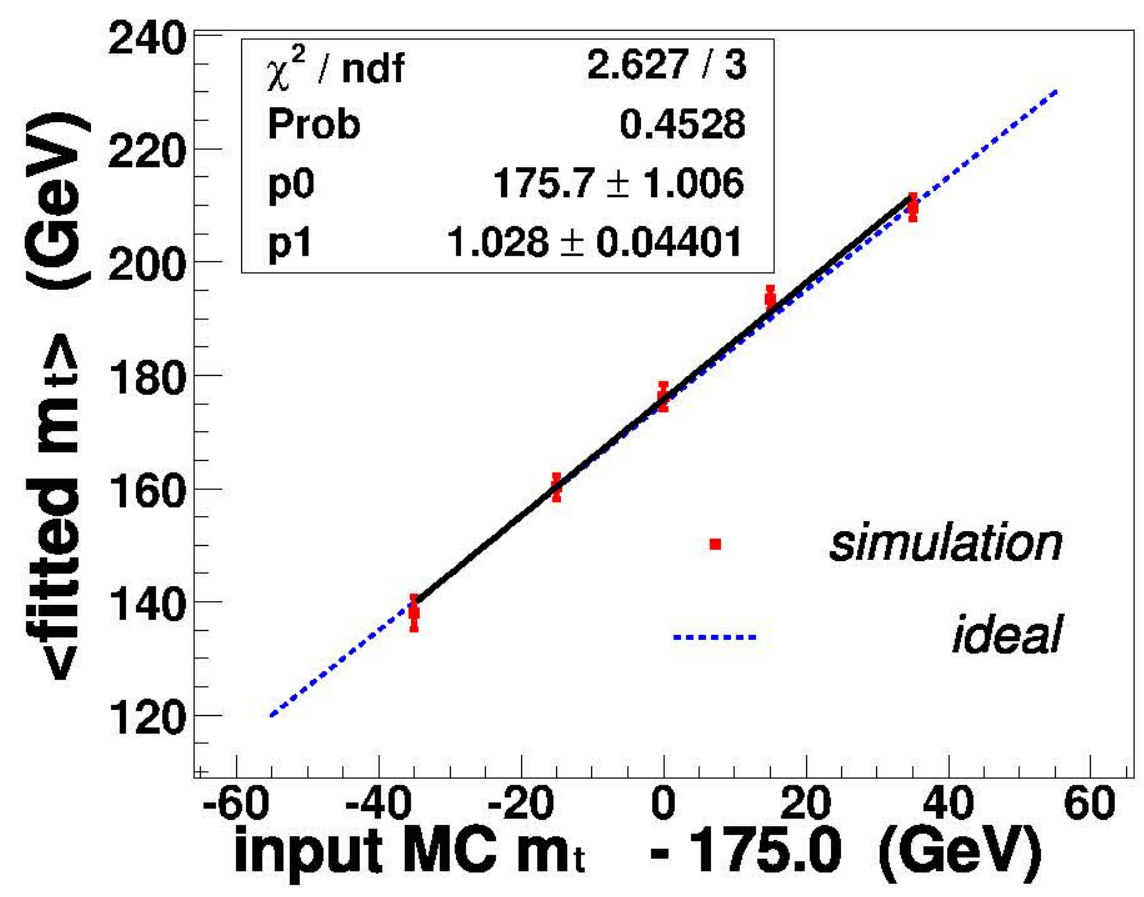

Figure 8.17: Calibration curve from simulated ensembles constructed using only signal di-clectron channcl events.

contamination. Figure 8.17 illustrates the best fit obtained from signal processes in the di-clectron channcl. $\Lambda$ fter the cnscmbles were contaminated with nearly one third of background processes, the best fit obtained is illustrated in Figure 8.18. In fact when ensembles have nearly $50 \%$ background type processes, then the slope of the straight line fit reduces by nearly $20 \%$ of its nominal value of unity ${ }^{7}$.

\footnotetext{
${ }^{7}$ The results are described in Appendix J.
} 


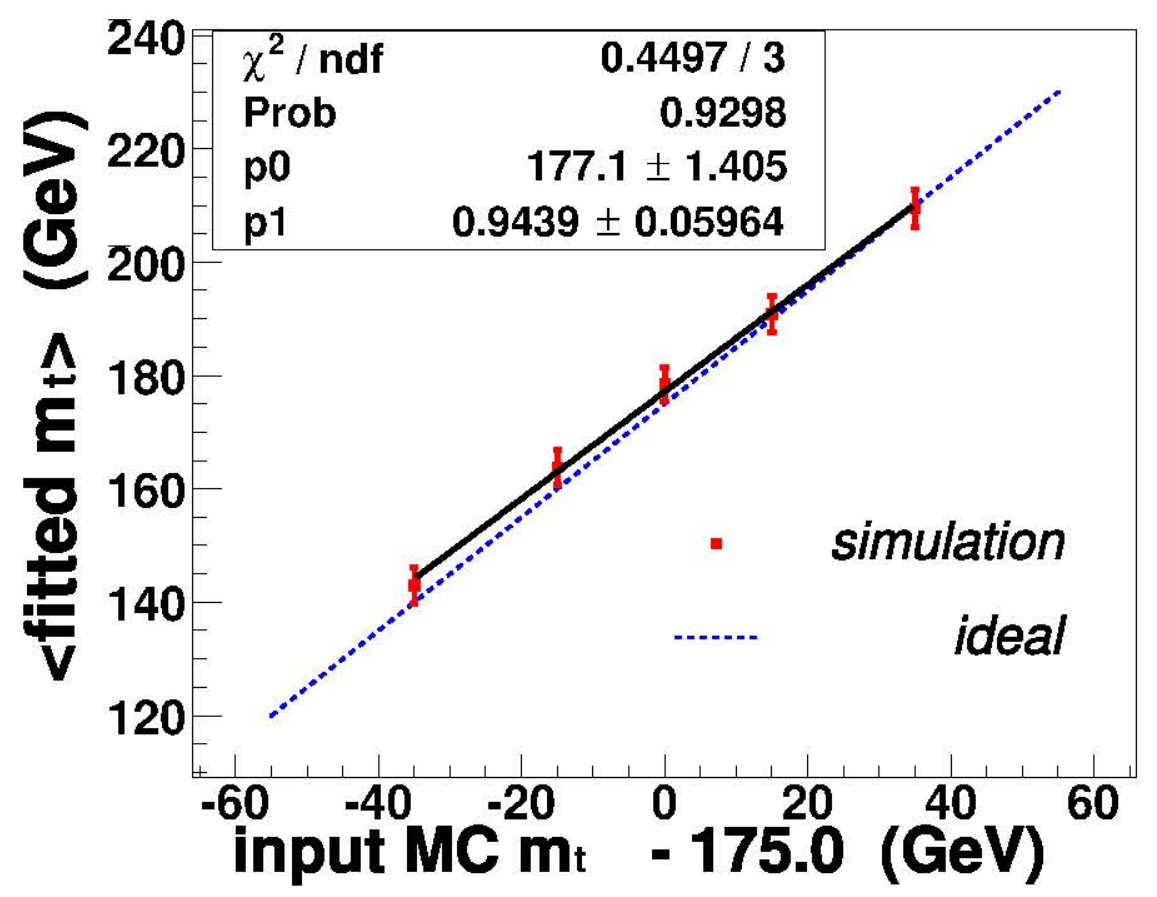

Figure 8.18: Calibration curve from simulated ensembles constructed using signal and background processes of the di-clectron channel. 


\subsection{The Maximum Likelihood Estimate using the data ensemble}

In this section the results of the Maximum Likelihood estimates from the data ensembles are presented.

\subsubsection{Results from the data ensembles: $\mathrm{e} \mu$ and ee channels}

\begin{tabular}{|r|r|r|}
\hline $\begin{array}{r}\text { input } \mathrm{MC} \mathrm{m}_{i} \\
(\mathrm{GeV})\end{array}$ & $\begin{array}{r}\text { negative } \\
\log \text { (likelihood) }\end{array}$ & $\begin{array}{r}\text { statistical } \\
\text { uncertainty }\end{array}$ \\
\hline 120.0 & 15.86 & 0.47 \\
140.0 & 13.26 & 0.35 \\
160.0 & 12.92 & 0.38 \\
175.0 & 13.85 & 0.25 \\
190.0 & 14.73 & 0.32 \\
210.0 & 16.36 & 0.21 \\
230.0 & 17.37 & 0.21 \\
\hline
\end{tabular}

Table 8.6: Log-likelihood versus input MC $m_{t}$ for the $e \mu$ cnscmble.

\begin{tabular}{|r|r|r|}
\hline $\begin{array}{r}\text { input } \mathbf{M C ~ m}_{t} \\
(\mathbf{G e V})\end{array}$ & $\begin{array}{r}\text { negative } \\
\log (\text { likelihood) }\end{array}$ & $\begin{array}{r}\text { statistical } \\
\text { uncertainty }\end{array}$ \\
\hline 120.0 & 8.58 & 0.62 \\
110.0 & 7.33 & 0.48 \\
160.0 & 7.01 & 0.57 \\
175.0 & 7.19 & 0.37 \\
190.0 & 7.64 & 0.47 \\
210.0 & 8.69 & 0.36 \\
230.0 & 9.70 & 0.31 \\
\hline
\end{tabular}

Table 8.7: Log-likclihood versus input MC $m_{t}$ for the ee ensemble.

The Table 8.6 lists the logarithm of the likelihood obtained for various input

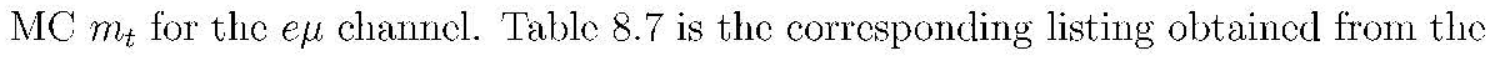


ee cnsemble. Figure 8.19 and 8.20 are the logarithm of the likelihood plots as a function of the input mass of the top quark using the data ensemble for the $e \mu$ and the di-clectron channels respectively. The Maximum Likclihood Estimates (MLE) are the the minima of the functions which best (numerically) fit to the two negative log-likelihood distributions.

The MLE (from Figure 8.19) of the mass of the top quark from the ensemble of 8 events selected in the $e \mu$ channel is:

$$
m_{t}=153 . \bar{\jmath}_{-14.9}^{+17.1} \text { (stat.) GcV. }
$$

The corresponding MLE (from Figure 8.20) obtained for the 5 events selected in the di-electron channel is:

$$
m_{t}=158.6_{-22.3}^{+25.6} \text { (stat.) GoV. }
$$

While the consistency checks described in the previous section indicate that there is no need for a bias correction in the case for the e $\mu$ channel, a correction to eliminate the bias is applicd in the di-clectron channel. The corrected MLE is presented in the next sub-section.

Figure 8.21 represents the normalized template distribution for the mass that fits the data best. Here, the template with signal events having an input MC $m_{t}=160$ $\mathrm{GeV}$ is used. Superposed on the template histogram is the normalized histogram of $m_{\text {peak }}$ values from the cight $e \mu$ candidate cvents.

Figure 8.22 represents the normalized template in the ee channel for an input value of the mass of the top quark of $160 \mathrm{GeV}$. A normalized histogran of the $m_{\text {peak }}$ values obtained from the five candidate events are superposed over the template distribution. 


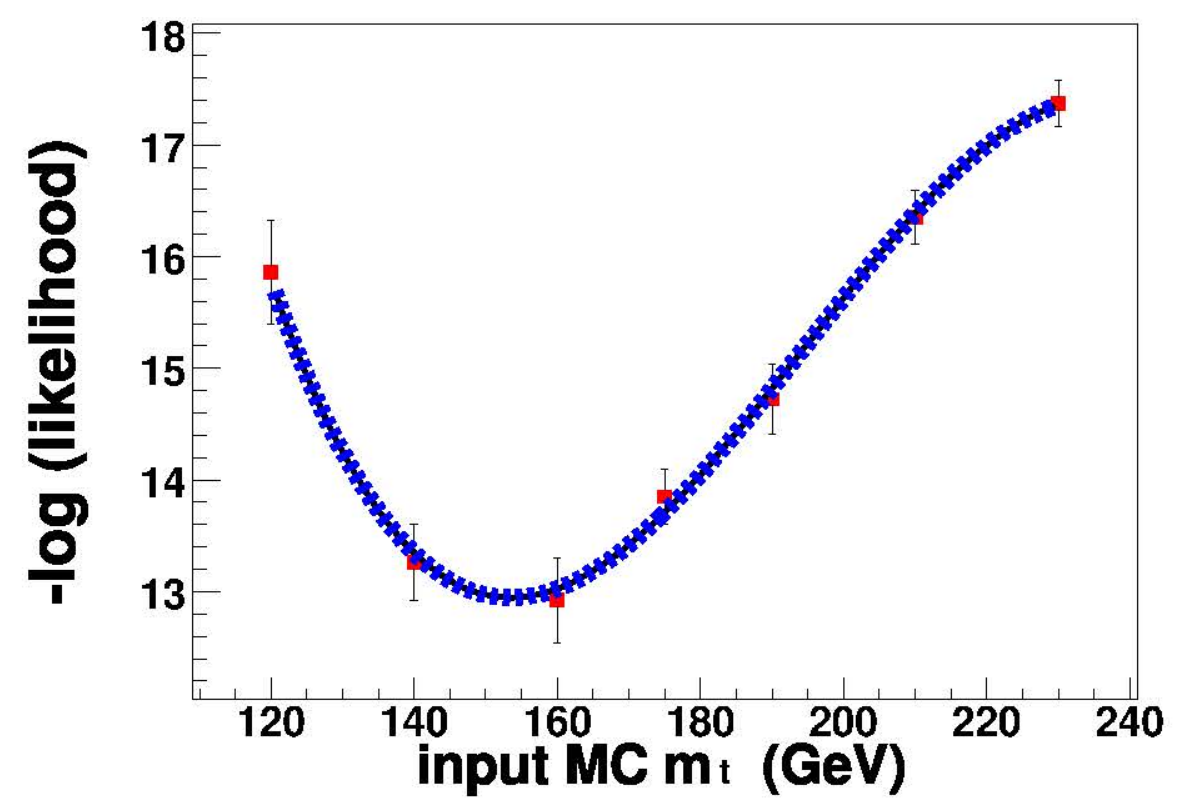

Figure 8.19: The maximum likelihood fit to the $e \mu$ data ensemble.

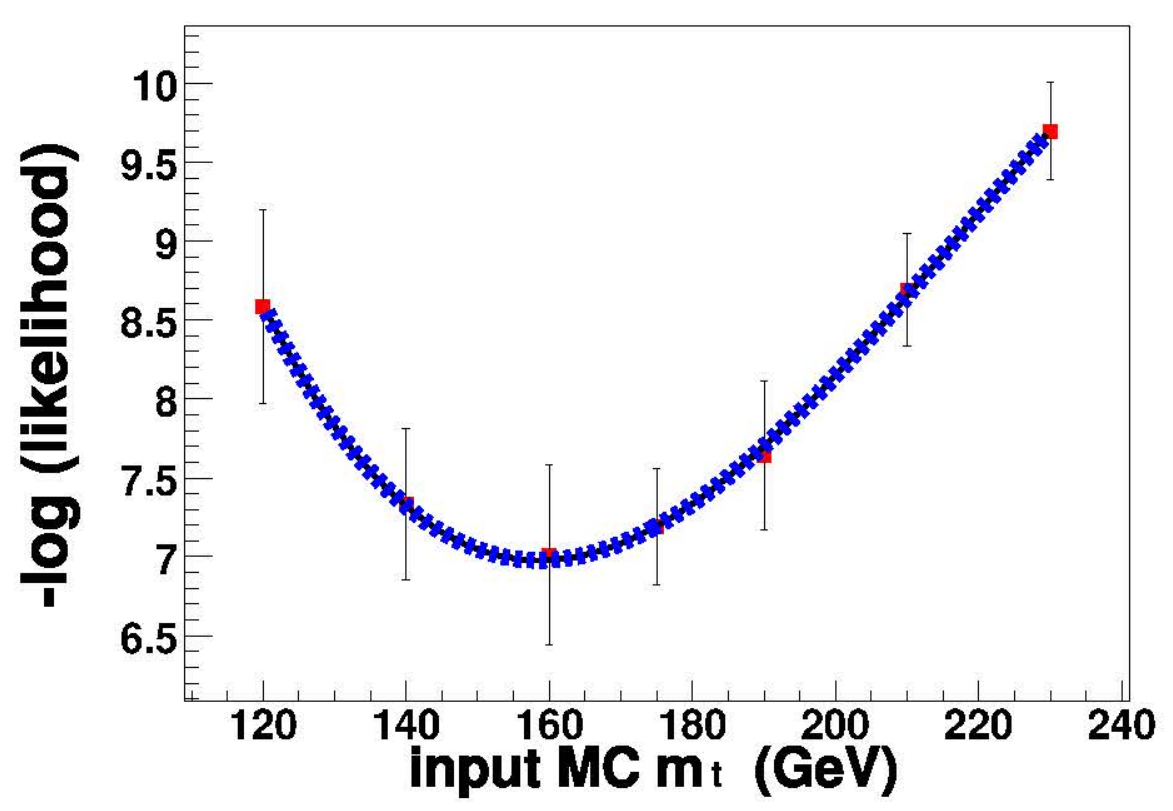

Figure 8.20: The maximum likelihood fit to the di-electron data ensemble. 


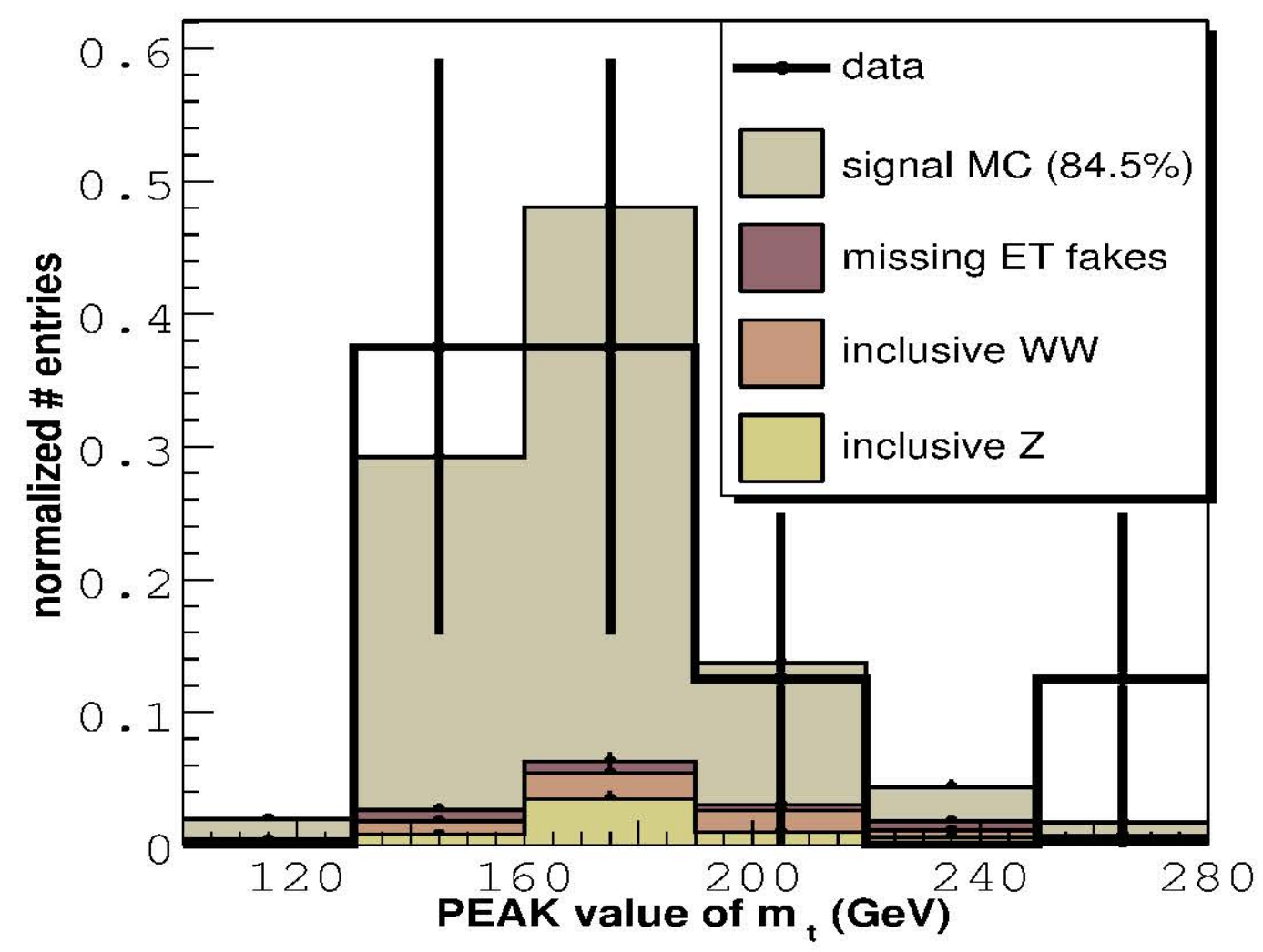

Figure 8.21: The combined signal (input $m_{t}=160 \mathrm{GcV}$ ) and background template for the $e \mu$ channel that fits the data best. Superposed on the normalized template is the normalized $e \mu$ ensemble histogram with the $m_{p e a k}$ values from the eight candidate events. 


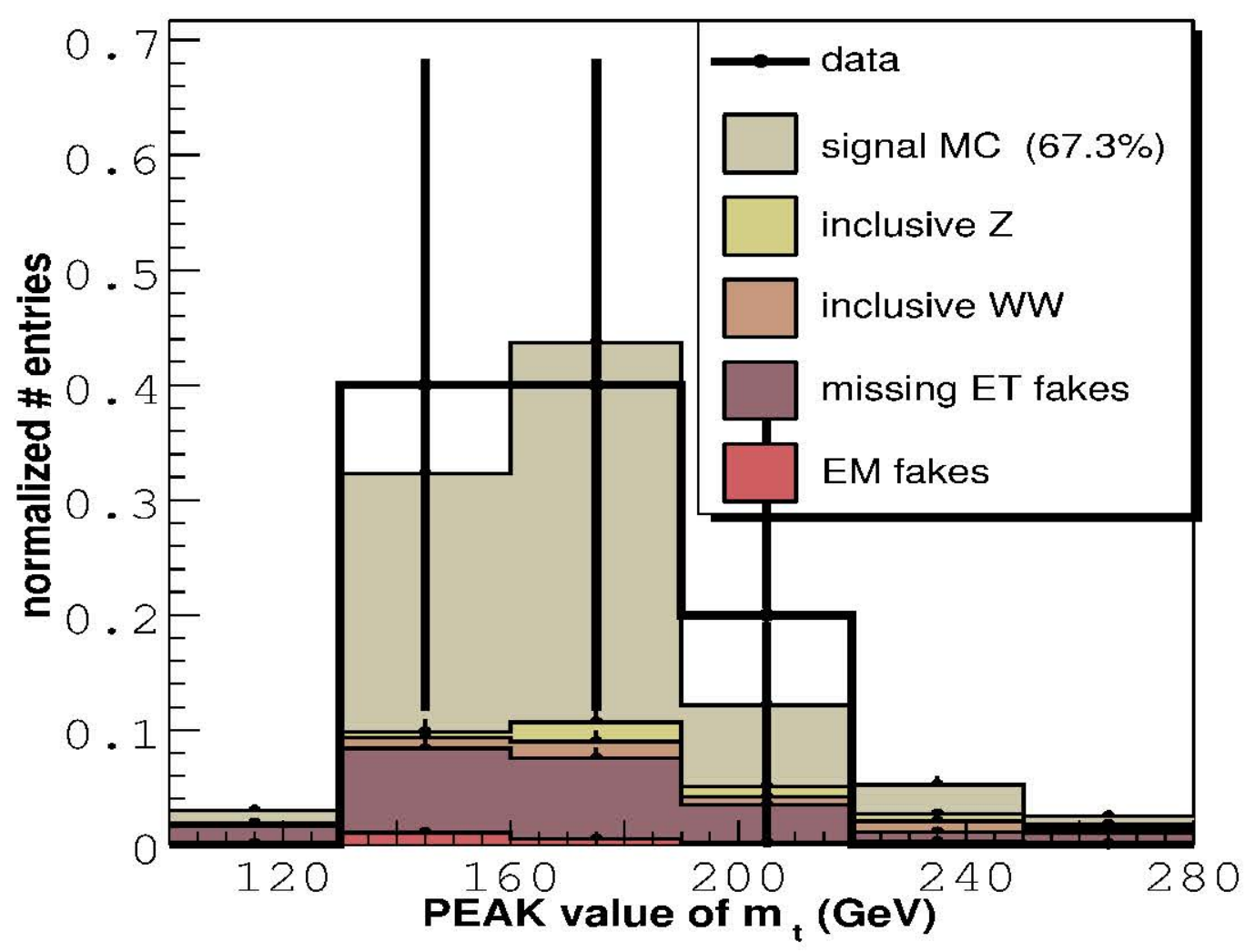

Figure 8.22: The combined signal (input $m_{t}=160 \mathrm{GcV}$ ) and background template for the ee channel that fits the data best. Superposed on the normalized template is the normalized ee ensemble histogram with the $m_{p c a k}$ values from the five candidate events. 


\subsubsection{The Maximum Likelihood Estimate of the di-electron data ensemble with the calibration bias correction}

The calibration curve from Figure 8.18 is used to correct the most likely value of the mass of the top quark obtained from ensembles. The di-electron data ensemble gives the best numerical fit shown in Figure 8.20. as well as the top plot on Figure 8.23. After the application of the bias correction, the same numerical fit now translates into the fit shown on the bottom plot of Figure 8.23. 'The MLE we now obtain for the di-electron data ensemble is:

$$
m_{t}=155.4_{-23.6}^{+27.1} \text { (stat.) GeV. }
$$



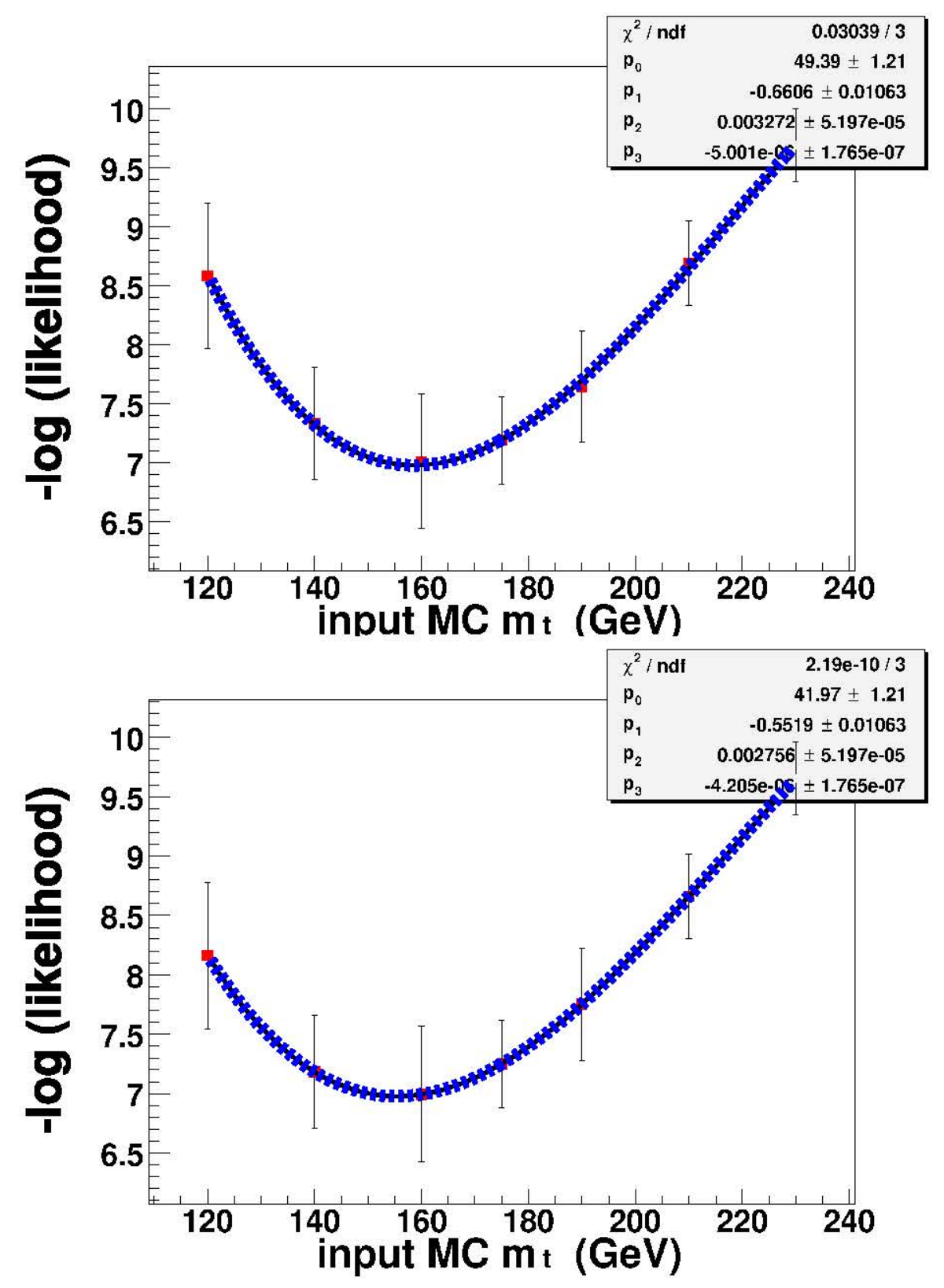

Figure 8.23: (Top) The parameterized log-likelihood distribution as a function of the input $\mathrm{MC}$ top quark mass for the ensemble of di-electron events. This plot is identical to the Figure 8.20.

(Bottom) The log-likelihood distribution as a function of the input MC value of the top quark mass, for the cnscmble of di-clectron events. This plot is obtained after the application of the bias correction derived from the calibration curve in Figure 8.18. 


\subsubsection{A caveat}

Consider the distributions of MLEs using simulated events with detector resolution effects. Using ensembles with signal events with an input mass of the top quark of $175 \mathrm{GeV}$, the question to be addressed is:

how likely is it that the algorithm yields a measured mass which is $<160 \mathrm{GeV}$ ?

For this study each one of these ensembles has the nominal background composition as well (Table 8.1). The simulations for the $e \mu$ channcl have 8 events per cnscmble, while those for the di-electron chamnel have 5 events per ensemble.

Figure 8.24 is a distribution of the MLEs for the $e \mu$ channel, and Figure 8.25 is that from the di-electron channel. It has been ensured that in each of these tests, all ensembles have unique events ${ }^{8}$. From both distributions nearly $15-20 \%$ of the total enscmbles yicld MLEs having valucs less than $160 \mathrm{GeV}$. It must be noted that this value is dependent on the bin width of the respective histograms.

\footnotetext{
${ }^{8}$ No ensemble is created after the re-shuffling of events.
} 


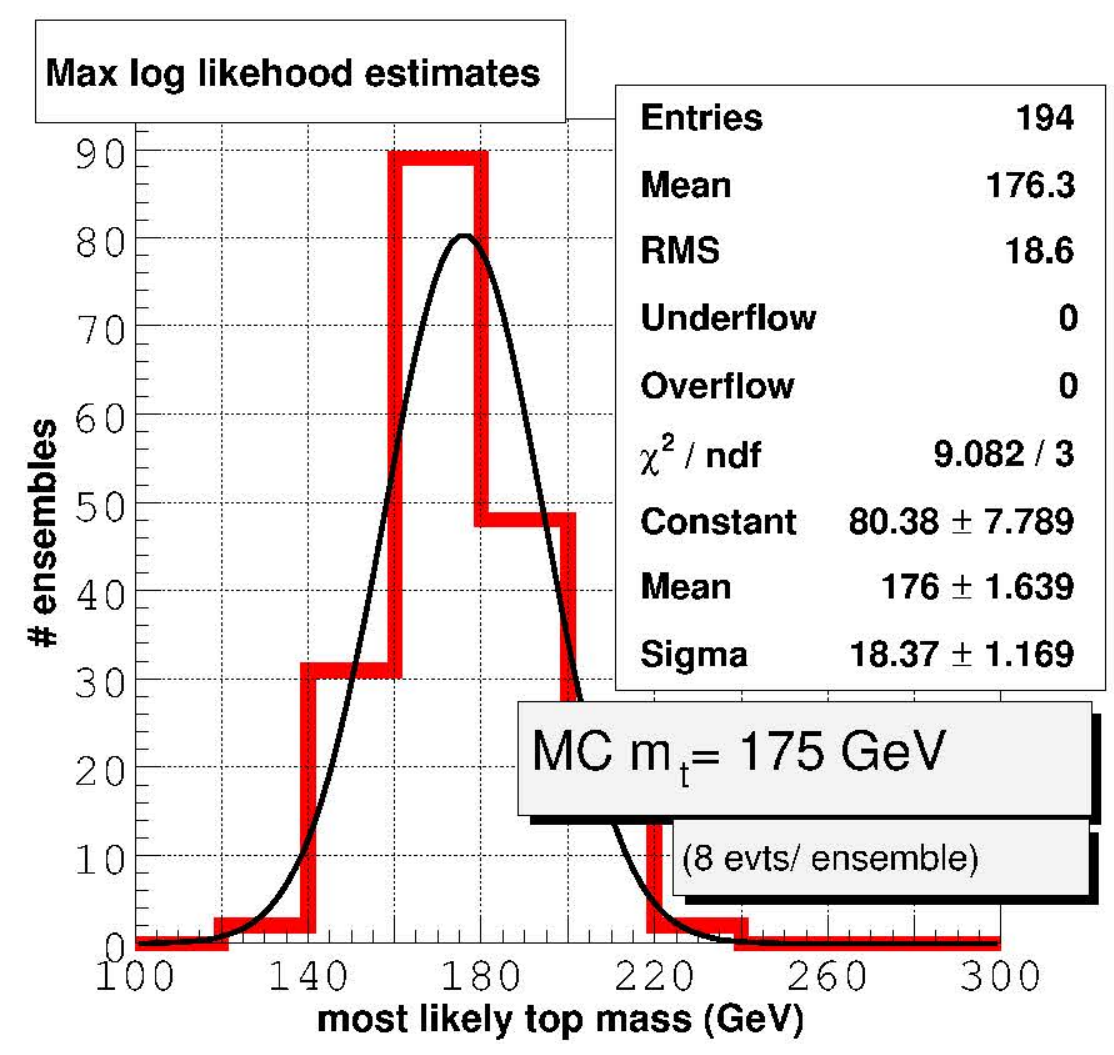

Figure 8.24: The distribution of MLEs from unique and simulated e $\mu$ cnscmbles, the signal cvents having input $\mathrm{MC} m_{t}=175 \mathrm{GoV}$. 


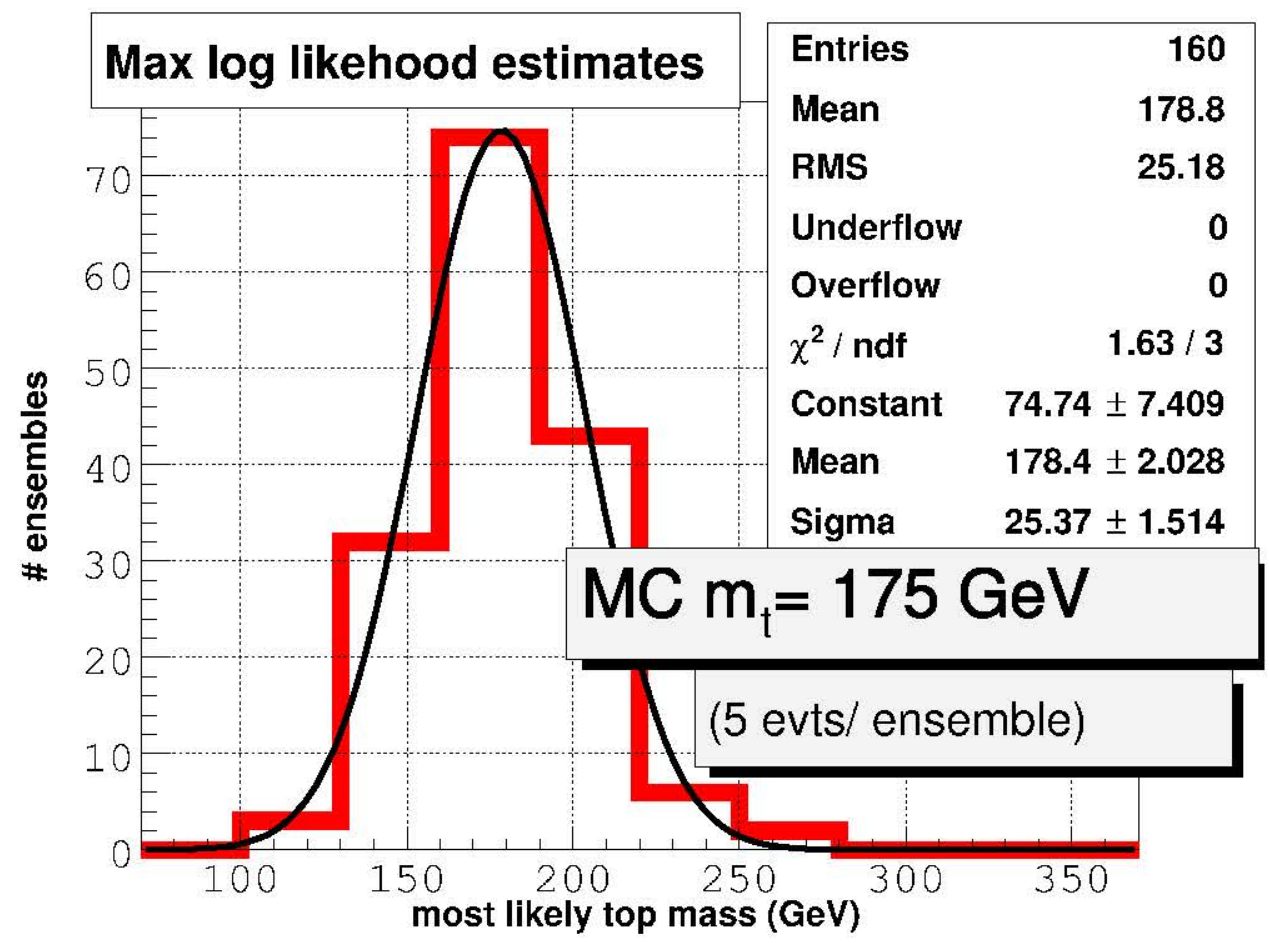

Figure 8.25: The distribution of MLEs from unique and simulated ee cnscmbles, the signal events having input MC $m_{t}=175 \mathrm{GcV}$. 


\subsection{Combined log-likelihood distributions}

In order to combine the results, functions from Figure 8.19 and the bottom plot on Figure 8.23 (which give the best estimate of the value of the mass of the top quark in each of the two channels) are added. Figure 8.26 represents the combined $\log$-likelihood as a function of the input value of the mass. The VLE obtained from this combined numerical fit represents the most likcly estimate of the top quark for the ee and e ensembles. The Maximum likelihood Fstimate for the combined cnscmbles and the associated statistical uncertainty is:

$$
154.1_{-12.8}^{+14.2} \text { (stat.) GeV. }
$$

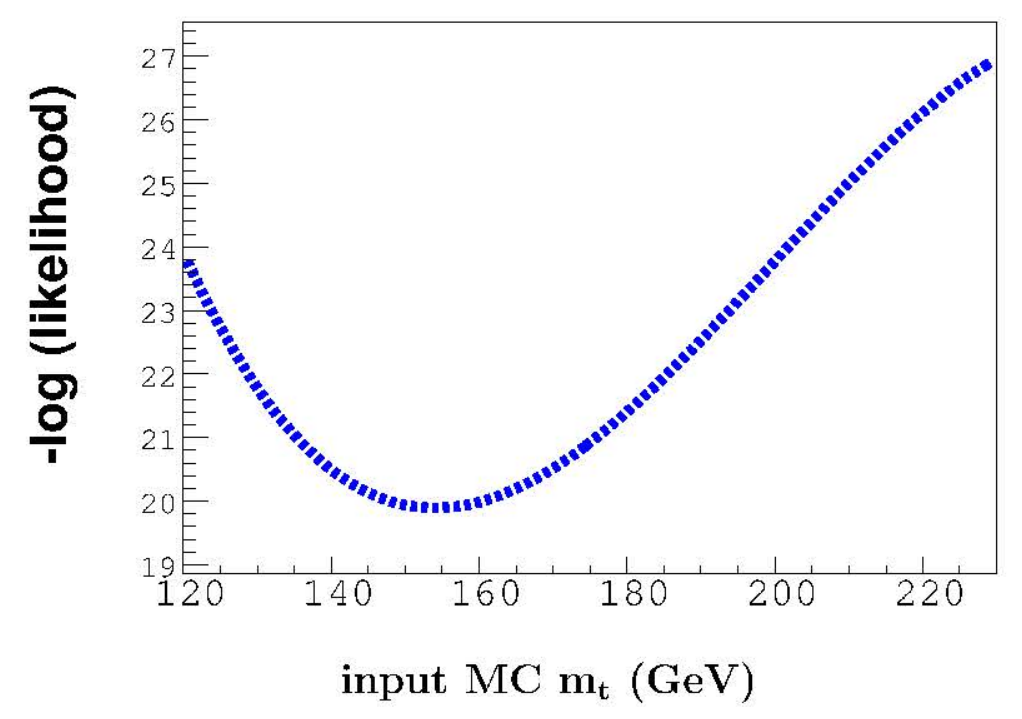

Figure 8.26: 'l'he combined log-likelihood distribution for the $e \mu$ and the di-clectron data cnscmbles. 


\subsection{Systematic uncertainties}

Using ensembles of simulated events, the primary systematic uncertainties are established. A comprehensive listing of the associated uncertainties is illustrated in Table 8.10. From previous studies[27] we know that the systematic uncertainty in determining the energy scale of jets is the dominant contribution to the overall systematic uncertainty in this measurement ${ }^{9}$. The other sources of systematic uncertainties are from Monte Carlo simulations with multiple parton interactions. The usc of Alpgen along with Pythia, for signal event generation, as opposed to another generator, e.g. Herwig, may introduce a systematic bias. This effect is explored as well. The issue of systematic uncertainty being introduced due to the finite statistics is also addressed.

In the sub-sections which follow, we discuss the above-mentioned sources of systematic uncertainties associated with the measurement of the mass. For estimating every systematic uncertainty, ensembles of simulated events were specially produced incorporating the physical effect under study. The ensembles were then fitted using the nominally produced templates of simulated events.

\subsubsection{The jet energy scale}

For estimating the unecrtainty in the detcrmination of jet energy scale, the jet's 4-vector from every selected event is fluctuated by an amount $\Delta E$ that corresponds to its combined systematic and statistical uncertainty[91]. For estimating the upper limit on the uncertainty, the 1-vectors of the jets are increased by the definite amount $\Delta E$, whereas for estimating the lower limit it is decreased by $\Delta E$.

\footnotetext{
${ }^{9}$ This uncertainty has a larger effect in the case of the measurement of the top quark mass in the single lepton + jets channel, since there are at least 4 jets associated with every event.
} 


\begin{tabular}{lr}
\hline $\begin{array}{l}\text { source of } \\
\text { systematic }\end{array}$ & uncertainty \\
uncertainty & $(\mathrm{GeV})$ \\
\hline \hline (correlated) & \\
\hline calibration of 4-vectors & \\
(at $150 \mathrm{GeV})$ & +5.5 \\
jet 4 -vector & \\
(at $178 \mathrm{GeV})$ & \\
jet 4 -vcctor & \\
\hline physics processes & \\
multiple parton interactions (tuneA) & \\
event generators (fast MC) & 0.9 \\
parton distribution functions & 1.0 \\
\hline \hline (un-correlated ) & $\left(=\Delta_{\text {stat. }}\right)$ \\
\hline ensemble calibration curve & \\
\hline & $0.05 \pm \Delta_{\text {stal. }}$ \\
\hline
\end{tabular}

Table 8.8: A summary of various systcmatic uncertaintics associated with the mass measurement in the $e \mu$ channel. The results have been estimated for input $m_{t}=$ $175 \mathrm{GeV}$ (unless otherwise specified).

Two distinct tests are performed to establish the jet energy systematic uncertainty. In the first test, the ensembles as well as template histograms are constructed only from the signal process, for all input values of the mass of the top quark. The 4-vectors of the jets used in the analysis are fluctuated as just mentioned above. In the sccond test, both the cnsembles and templates are constructed from signal as well as background processes. Then the 4-vectors of the jets in both signal and background processes used in the analysis are fluctuated as in the former case.

All results are derived from the series of calibration plots illustrated in this section. Figure 8.27 and Figure 8.28 represent the calibration curves for the $(\epsilon \mu)$ cnscmbles with events whose jet energy scale is increased and decreased by one 


\begin{tabular}{lr}
\hline $\begin{array}{l}\text { source of } \\
\text { systematic } \\
\text { uncertainty }\end{array}$ & uncertainty \\
\hline \hline (correlated) & $(\mathrm{GeV})$ \\
\hline calibration of 4-vectors & \\
(at 150 GeV) & \\
jet 4-vector & ${ }_{-3.4}^{+1.1}$ \\
(at 178 GeV) & +2.0 \\
jet 4-vector & ${ }_{-4.1}$ \\
\hline physics processes & 1.0 \\
multiple parton interactions (tuneA) & 3.0 \\
event generators (fast MC) & 0.9 \\
parton distribution functions & 2.2 \\
\hline \hline (un-correlated ) & $\left(=\Delta_{\text {stat. }}\right)$ \\
\hline ensemble calibration curve & $0.6 \pm \Delta_{\text {sial. }}$ \\
\hline
\end{tabular}

Table 8.9: A summary of various systematic uncertainties associated with the mass measurement in the di-electron channel. The results have been estimated using input $m_{t}=175 \mathrm{GeV}$ (unless otherwise specified).

standard deviation from the nominal value. Figures 8.29 and 8.30 represent studies of a similar nature, when the templates and ensembles have contribution of both signal as well as background processes. The actual uncertainty is obtained from the calibration curve for the measured value of the data ensemble. From both studies, signal-only studies and studies with signal and background, we estimate results which are consistent with each other, and are $\sim 5 \mathrm{GeV}$. Since the ensembles used in these studics are common, the systematic uncertainties are expected to be completely co-rclated.

Figure 8.31 and Figure 8.32 represent the calibration curves for ensembles with events whose jet energy scale is increased and decreased by one standard deviation from the nominal value. Since the nature of this source of systematic uncertainty is 
common to the $e \mu$ channel, we obtain results which are consistent with the previous analysis. 


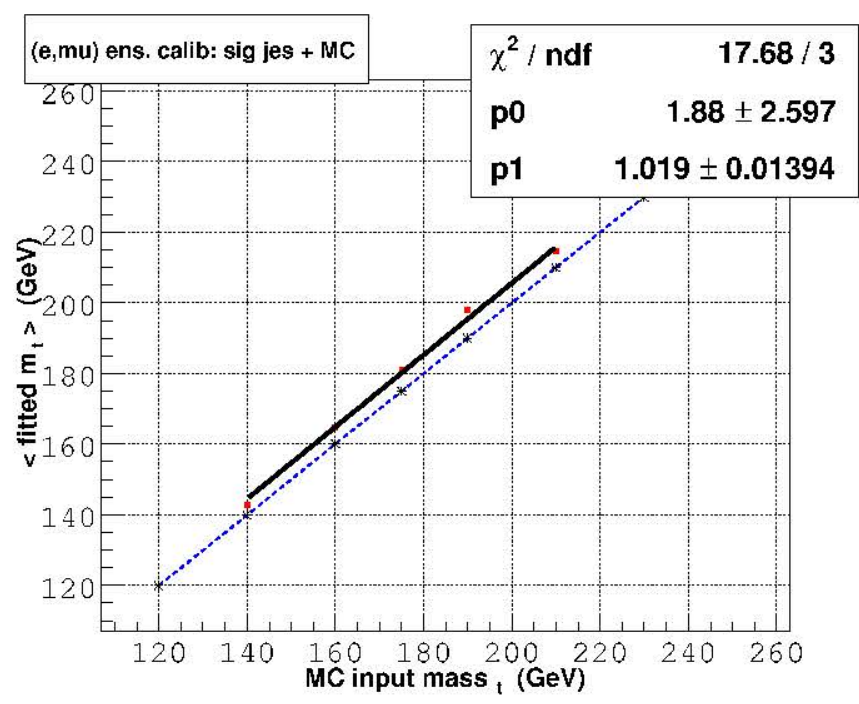

Figure 8.27: Calibration curve from ensembles of simulated events from the $e \mu$ channel, the jet energy from the leading jets have been scaled additionally by $\Delta \sigma_{E}$ with respect to the nominal jet energy calibration scale.

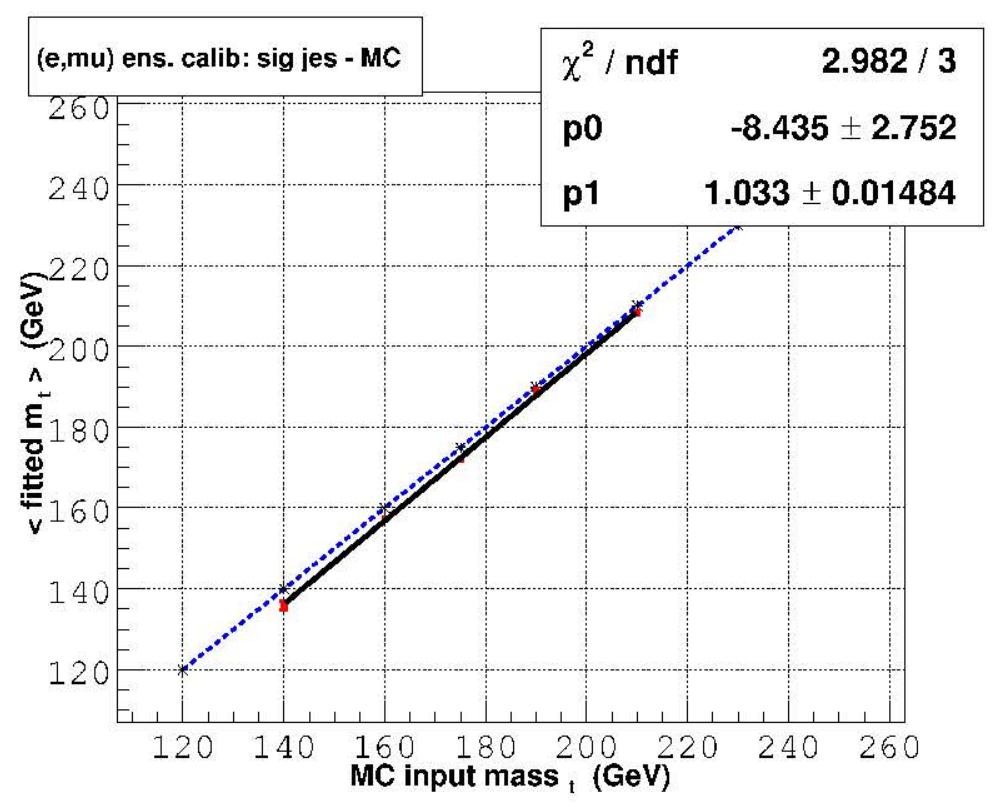

Figure 8.28: Calibration curve from ensembles of simulated events from signal $e \mu$ processes, the jet cnergy from the leading jets have becn reduced by $\Delta \sigma_{E}$ with respect to the nominal jet encrgy calibration scalc. 


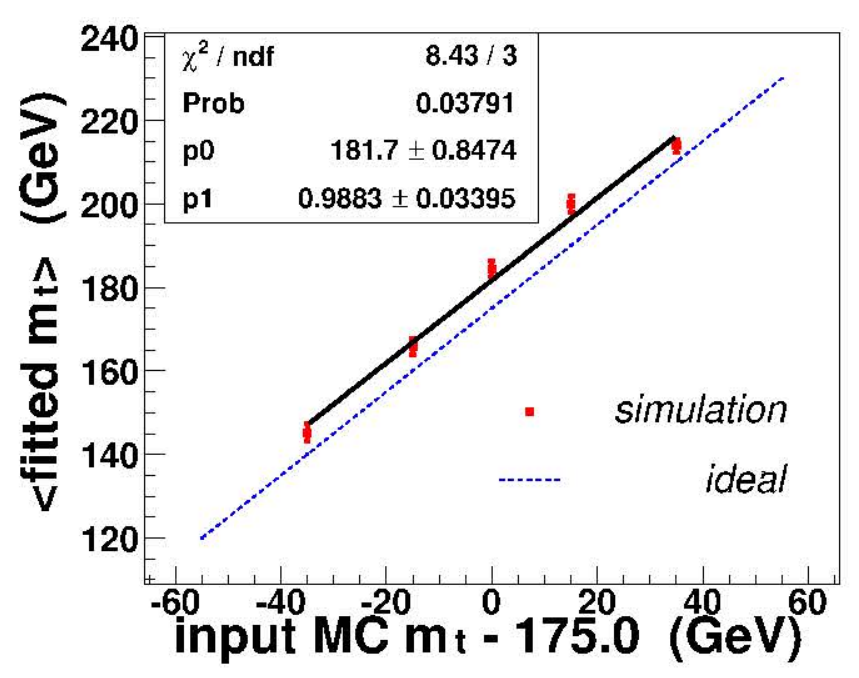

Figure 8.29: Calibration curve from ensembles of simulated events from the signal and background $e \mu$ processes, the jet energy from the leading jets have been scaled by $+\Delta \sigma_{E}$ with respect to the nominal jet energy calibration scale.

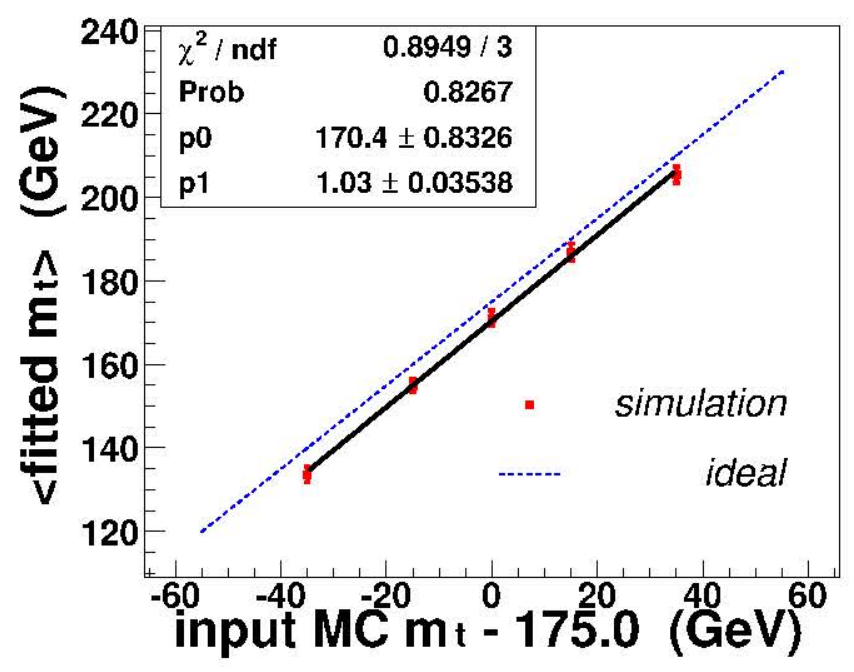

Figure 8.30: Calibration curve from cnsembles of simulated events from signal and background $e \mu$ processes, the jet energy from the leading jets have been reduced by $\Delta \sigma_{E}$ with respect to the nominal jet energy calibration scale. 


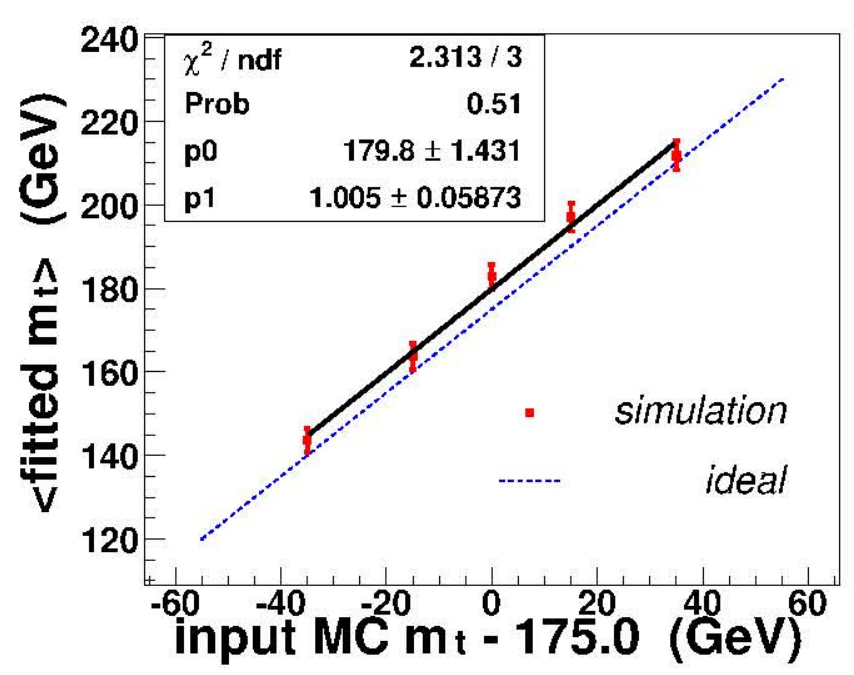

Figure 8.31: Calibration curve from ensembles of simulated events from the dielectron channel, the jet energy from the leading jets have been scaled by $+\Delta \sigma_{E}$ with respect to the nominal jet energy calibration scale.

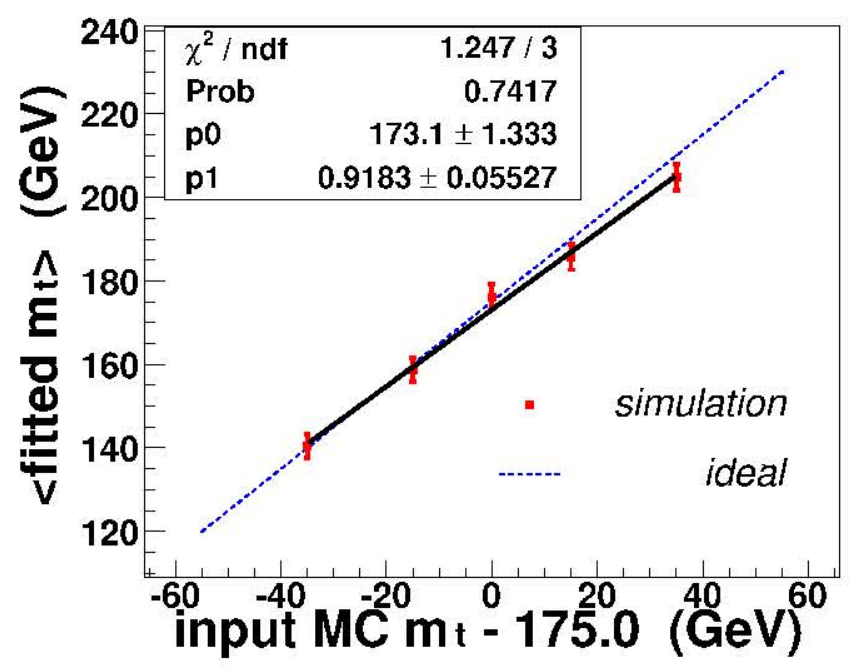

Figure 8.32: Calibration curve from ensembles of simulated events from signal dielectron processes, the jet energy from the leading jets have been scaled by $-\Delta \sigma_{E}$ with respect to the nominal jet energy calibration scale. 


\subsubsection{Electron energy and muon momentum scale}

The precision with which we know the electron energy scale[63] is much better than that of the muon momentum scale[67], which in turn in much better than that of the jet energy scale ${ }^{10}[69]$. It can be estimated that for an electron (muon) of nearly $50 \mathrm{GeV}$, the uncertainty in energy (or momentum) measurement is $\sim 2 \mathrm{GeV}$. For a jet of corresponding energy the uncertainty in energy determination may be upto nearly $1 \mathrm{GcV}$. Therefore the systematic uncertainty associated with the encrgy scale of the electrons and muons is small compared the systematic uncertainty associated with the jet energy scale.

\subsubsection{Multiple parton interactions}

All simulated events used for the analysis have incorporated single parton interactions only. 'To understand the eflects of the bias due to this, special signal events were generated incorporating the full detector resolutions for an input top mass value of $175.0 \mathrm{GeV}$. Ensembles construeted from these were then used to estimate the magnitude of the uncertainty. The measured value of this effect is $1 \pm 1 \mathrm{GeV}[78]$.

\subsubsection{Signal event generator}

We use simulated cvents generated by Pythia[11] as well as Herwig[15] for estimating this uncertainty. Templates constructed using events generated with Pythia, were used to obtain the Maximum Likelihood Estimate from cnscmbles cvents constructed using Herwig LO and NIOO samples. The uncertainty on the measurement of the mass of the top quark due to this effect was measured to be about $3 \mathrm{GeV}[78]$.

\footnotetext{
${ }^{10}$ For simplicity we assume the region of interest for the energy-momentum scale of 4 -vectors is from $15 \mathrm{GeV}$ to nearly $100 \mathrm{GeV}$
} 


\subsubsection{Ensemble Calibration Curve}

The uncertainty due to the statistical fluctuations of the constituent points on the calibration curve was estimated at $150 \mathrm{GeV}$ and $178 \mathrm{GeV}$ for the two channels. The results were consistent with the statistical size of the available simulated ensembles. For the $e \mu$ case, the estimated uncertainty due to calibration was $1.3 \mathrm{GeV}$, while that for the di-electron channel it was $2.2 \mathrm{GeV}$. The uncertainties $\left(=\Delta_{\text {stat }}\right.$. $)$ in the two channcls are uncorrclated.

\subsubsection{Signal and background estimation}

Ensembles with the background composition increased by one standard deviation with respect to the (nominal) predetermined background composition were used for this test. These ensembles were using in conjunction with the nominal templates, and the results were calibrated just as before, for the nominal case. An estimate of $0.6 \pm \Delta_{\text {stat. }}$ was obtained for the di-electron channel, while the corresponding uncertainty for the e $\mu$ channel was determined to be $0.05 \pm \Delta_{\text {stat }}$. This uncertainty is also uncorrclated in the two channels. The uncertainty due to background contamination is much smaller compared to the one obtained due to the fit from the cnscmble calibration curve.

\subsubsection{Miscellaneous issues}

There are other issues which have systematic eflects on the measurement of the mass of the top quark. All these issues are small compared to that due to the uncertainty in the measurement of the jet energy. The systematic uncertainty due to the effects of trigger bias have not been included. Previous studies[92] in the e $\mu$ 
channel have shown that the eflect of this is $\sim 2 \mathrm{GeV}$. The systematic uncertainty due to a different higher order polynomial fit to the log-likelihood distributions have not been studied rigorously. Preliminary studies have shown that a 1 parameter (cubic) fit does not produce a significant systematic uncertainty than that from a 5 parameter fit.

\subsection{The combined systematic uncertainty}

\begin{tabular}{|l|rrr|}
\hline $\begin{array}{l}\text { source of } \\
\text { uncertainty }\end{array}$ & $\begin{array}{r}\text { e } \mu \text { channel } \\
(\mathrm{GeV})\end{array}$ & $\begin{array}{r}\text { ee channel } \\
(\mathrm{GeV})\end{array}$ & $\begin{array}{r}\text { combined } \\
(\mathrm{GeV})\end{array}$ \\
\hline statistical & ${ }_{-14.9}^{+17.1}$ & ${ }_{-23.6}^{+27.1}$ & ${ }_{-12.8}^{+14.2}$ \\
& & & 5.6 \\
\hline jet energy scale & & & 3.0 \\
event generation & & & 0.9 \\
parton distribution function & 1.3 & 2.2 & 1.0 \\
underlying cvent simulation & & 6.9 & 1.1 \\
cnscmblc calibration curvc & 6.6 & & 6.5 \\
combincd systcmatic & & & \\
\hline \hline & ${ }_{-16}^{+18}$ & ${ }_{-25}^{+28}$ & 15 \\
\hline
\end{tabular}

Table 8.10: A summary of the measured uncertainties associated with the mass measurement. These results are derived from Table 8.8 and Table 8.9

Previously, in Section 8.9 a combined statistical uncertainty in the measurement of the mass of the top quark in the two independent channels was discussed. This section highlights the combination of the systematic uncertainties in these channels.

The Table 8.10 highlights various uncertaintics from the previous section. The systematic uncertainties in the two independent chamels are consistent with each other. Ilowever, the systematic uncertainties determined from the ensemble tests 
in the di-clectron channel are less precise than those of the $e \mu$ channcl. This arises from the fact that the total number of simulated events generated in the di-electron channel are nearly hall of those in the $e \mu$ channel $^{11}$. Morcover, the size of the $e \mu$ ensemble is 8 events, whereas that of the ee ensemble is nearly half ( 5 events) as well. Since the underlying physics which gives rise to these uncertainties is identical in the two cascs, we primarily use those results which are more precise. The uncertainty due to the jet energy scale calibration is determined as the weighted average obtaincd in the e $\mu$ channel. This uncertainty, along with the contributions from multiple parton interaction, from the use of different event generators, differences in parton distribution functions are correlated uncertainties in the channels which are combined. The uncertainty due to the ensemble calibration curve and that from background estimation are the un-correlated systematic uncertainties in the two channcls.

\footnotetext{
${ }^{11}$ The $t \bar{t} \rightarrow$ (inclusive) di-leptons process is used
} 


\subsection{The measured mass}

Figure 8.33 illustrates the individual results in the $e \mu$ and the $e e$ channel as well as a combined measurement. These measurements are contrasted with the current world average as well as the Run I measurement from the dilepton channels.

The measured mass of the top quark from the di-electron ensemble and the $e \mu$ ensemble is:

$$
m_{t}=154.1_{-12.8}^{+14.2} \text { (stat.) } \pm 6.6 \text { (syst.) GeV. }
$$

\subsection{Salient features of the mass analysis}

In this measurement a total of thirteen events were used. The topological characteristics of these events matched that of events consistent with the Standard Model decay of $t \bar{t}$ via the di-lepton channel. This is the first measurement of the mass of the top quark in Run II in the di-lepton channel using the DØ detector.

A simplistic approach of using a single estimator per event is taken. However, the analysis preformed in Run I[27] used information from the shape of the weight distribution of events as well ${ }^{12}$.

It is interesting to note from Table 8.1 [in Section 8.4.5] that the number of candidate events obtained from collider data are more than we expect[52]. It is plausible that some or all of the excess events may not be signal processes. Therefore, for simulated ensemble tests, the total ensemble size is kept fixed ${ }^{13}$, while the number of the crents from signal and background processes are multinomially varicd about

\footnotetext{
${ }^{12}$ This procedure is computationally more intense and efforts are underway to obtain a measurement using this technique.

${ }^{13}$ This idea is different from the analysis done in Run I where the absolute number of background events was kept fixed.
} 
the nominal value.

More importantly, our understanding of the detector resolution and the application of various corrections (e.g. correcting jet 1-vectors to represent parton 1-vectors) represent an average value. Therefore, an ensemble with small number statistics is more prone to fluctuations than an ensemble with large number statistics.

For this analysis, information from the two leading $p_{T}$ jets in the event is used. Information from additional jets is neglected. From the 8 candidate events in the $e \mu$ channel, only one event has more than two jets. Evcnt \#8710859 in Run \#174901 has 4 jet objects with $p_{T}>15 \mathrm{GeV}$. From among the 5 candidate events in the $e e$ channel, 4 have only 2 jets each, while the fifth event (Event \#14448436, from Run \#180326) has 5 jets with $p_{T}>15 \mathrm{GeV}$. 


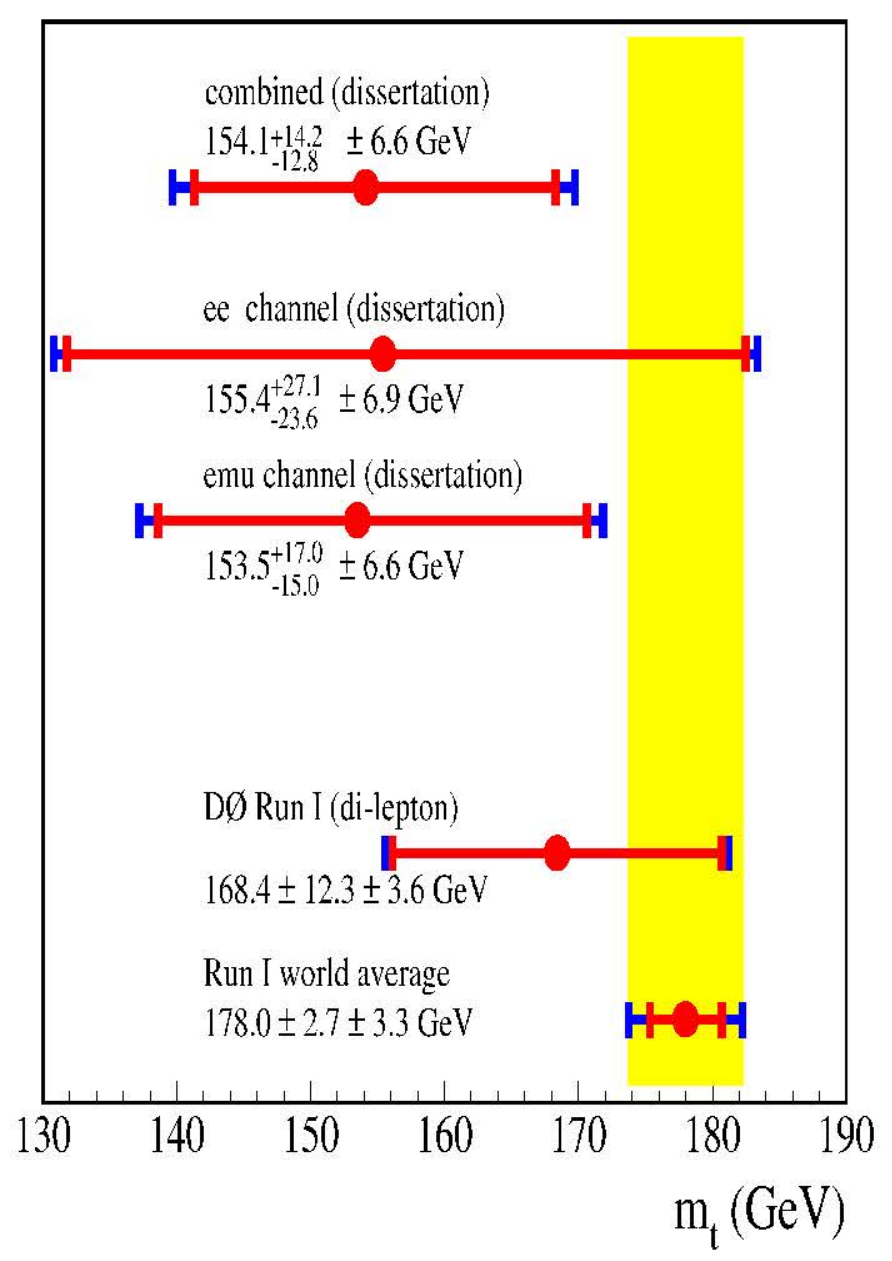

Figure 8.33: The combined results from this dissertation. As a comparison the measurements of the top quark mass in the di-lepton channel in Run I and the current (Run I) world average value are also illustrated. The inner error bar (red) is only due to the statistical uncertainty. The outer error bar is due to the combined statistical and systematic uncertainties. The shaded (yellow) region corresponds to the overall Run I world average measurement. 


\section{Chapter 9}

\section{Comparison With Other}

\section{Measurements}

This chapter describes the implications of the measurement of the mass of the top quark. The obtained result is first compared with other independent results of the mass of the top quark.

\subsection{Independent measurements in the di-lepton channel}

$\Lambda$ first, the result obtained in this analysis is contrasted with the other independent measurements in the di-lepton channel. Figure 9.1 illustrates that this measurement is consistent with other independent measurements in the di-lepton channel. This measurement is not within one standard deviation with respect to the Run I world average measurement of $178 \pm 2.7$ (stat.) \pm 3.3 (syst.) GeV. Ilowever, the measured value of the top quark is within two standard deviations from 


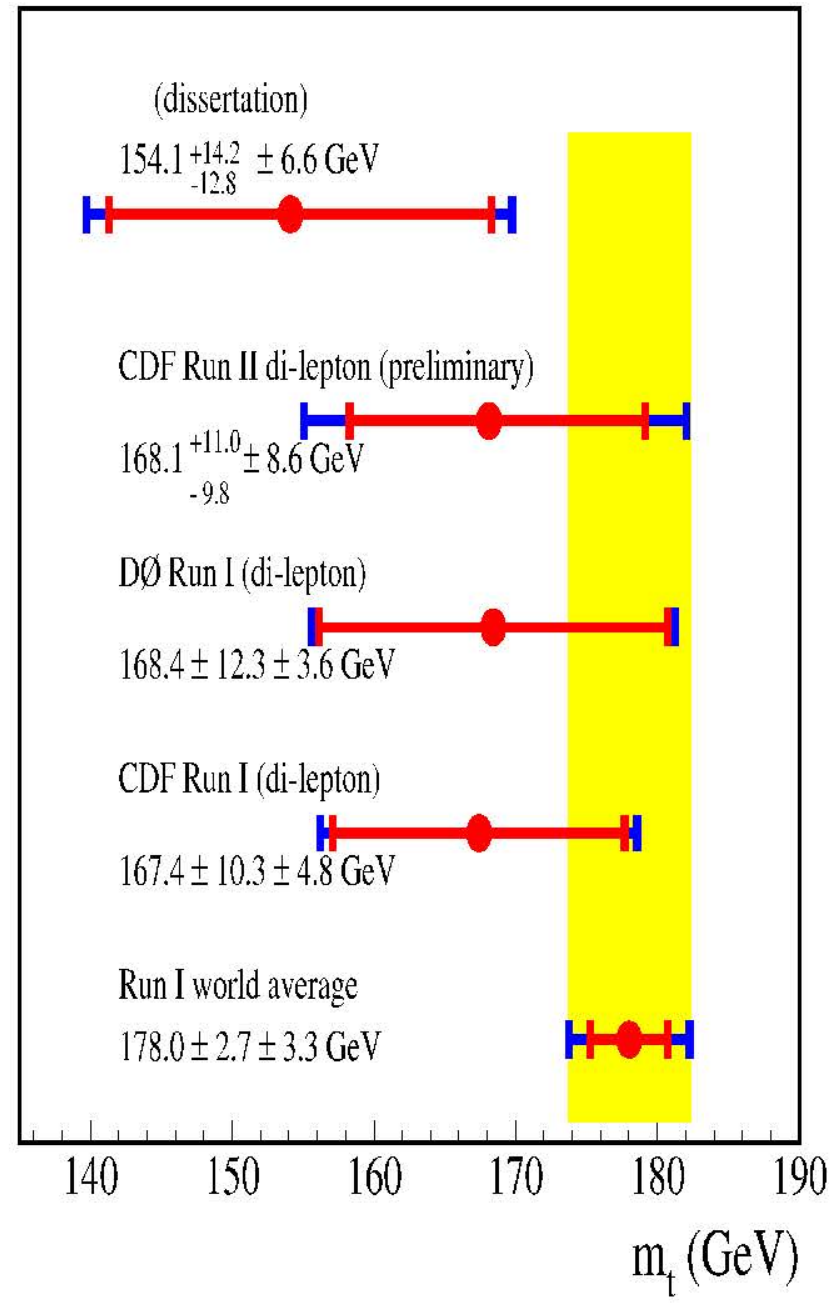

Figure 9.1: A comparative illustration of the measurements of the top quark in the di-lepton channel for the CDF and the DØ experiments. The inner error bar (red) is due to the statistical uncertainty. The outer crror bar is due to the combined statistical and systematic uncertaintics. The shaded (ycllow) region represents the ovcrall Run I world avcrage measurcment. 
the Run I world average measurement. This inconsistency may possibly be due to statistical fluctuations. The uncertainties in all di-lepton channel measurements are dominated by the statistical uncertainty (inner crror bar in the plot in Figure 9.1). While the world average measurement was determined using over two hundred candidate events from all the possible $t \bar{t}$ decay channels, only 13 candidate events were used for this measurcment. 


\subsection{Independent measurements from Run II}

This section deals with the current measurements of the top quark mass in Rum II from both the $\mathrm{D} \varnothing$ as well as the CDF collaborations. The CDF detector is located at the position $B \varnothing$ indicated on the Tevatron schematic in Chapter 4, Figure 4.1.

\subsubsection{Recent results from the $\mathrm{D} \varnothing$ experiment}

The DQ experiment has also measured[93] the mass of the top quark using the top and anti-top quark pairs which decay to a charged lepton (an electron or a muon) and at least 4 jets. While two of these jets are from the hadronization of the $b$-quark, the other two jets originate from the hadronic decay of the $W$-boson. Using a template method [94] the mass of the top quark was deter-

mined to be $170.0 \pm 6.5$ (stat.) ${ }_{-6.1}^{+10.5}$ (syst.) GoV. In an independent analysis, using the ideogram method[94] the mass of the top quark was measured to be 177.5 \pm 5.8 (stat.) \pm 7.1 (syst.) GoV. These results have been compared with previously obtained results in Rum I by the CDF and the DQ collaborations, as well as the Run I world average in Figure 9.2. 


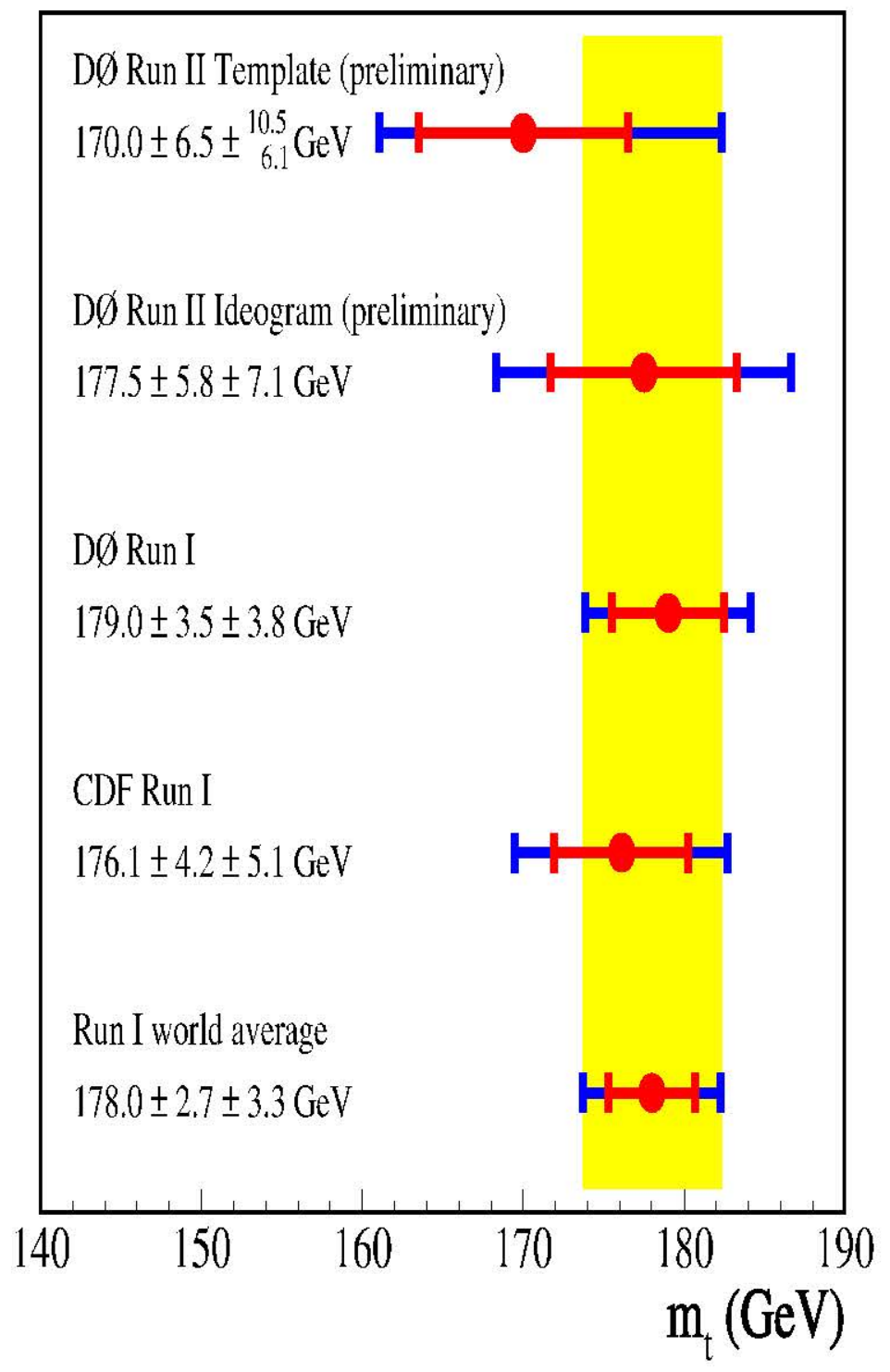

Figure 9.2: Measurements of the mass of the top quark from the CDF and the DØ collaboration. The inner error bar (red) is due to the statistical uncertainty. The outer error bar is due to the combined statistical and systematic uncertainties. The shaded (yclow) region represcnts the overall Run I world avarage measurement. 


\subsubsection{Recent results from the CDF experiment}

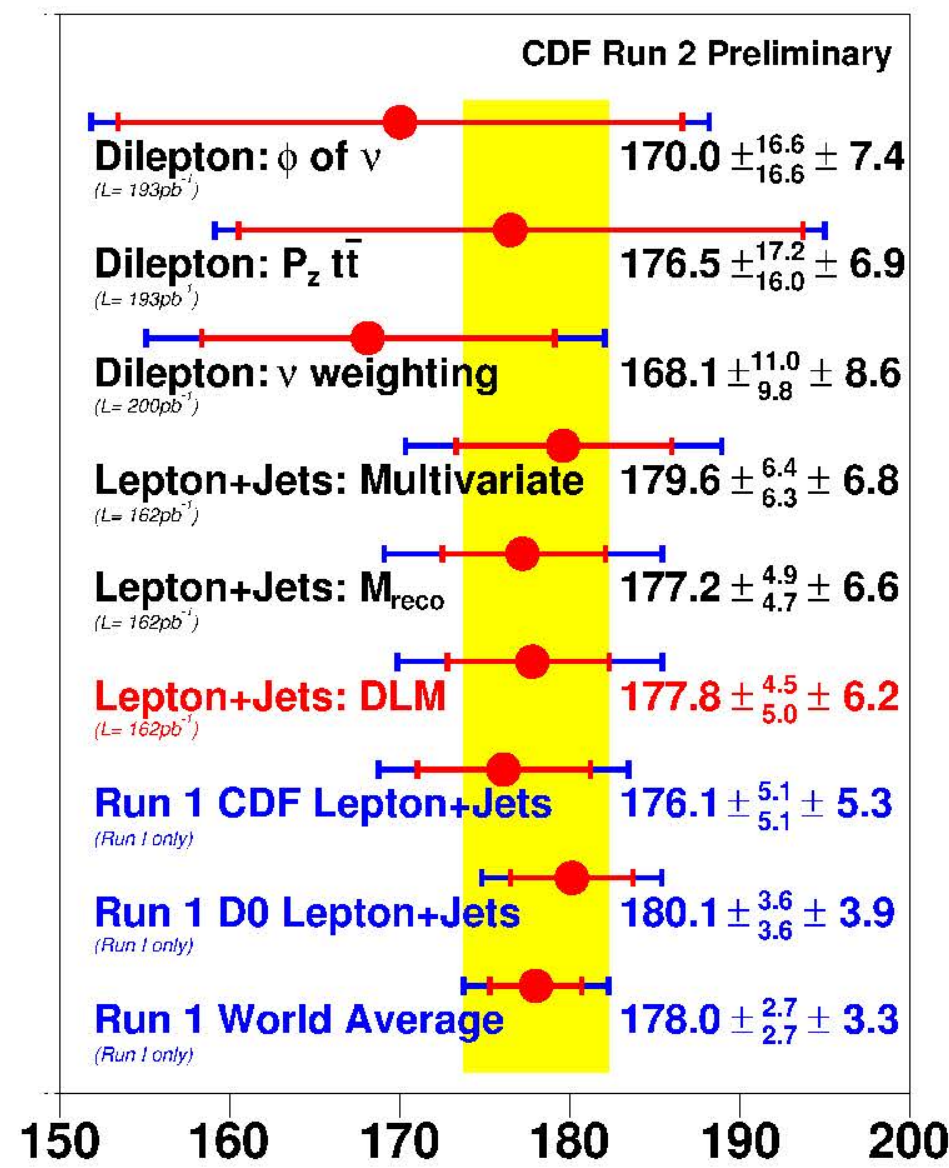

Figure 9.3: Measurements of the mass of the top quark from the CDF collaboration. The inner crror bar (red) is due to the statistical uncertainty. The outer crror bar is due to the combined statistical and systematic uncertaintics. The shaded (yollow) region represents the overall Run I world avarage measurement.

The CDF experiment has explored scveral different techniques for the measurement of the top quark mass. Figure 9.3 illustrates all independent measurements of the mass of the top quark by the CDF collaboration. The combined (preliminary) CDF Run II result is $177.8_{-5.0}^{+4.5}$ (stat.) \pm 6.2 (syst.) GeV[95]. Nearly $162 \mathrm{pb}^{-1}$ of Run II data (from March 2002 until September 2003) was used to obtain the result. 


\section{Chapter 10}

\section{Conclusion and future outlook}

$\Lambda$ dynamical likelihood method is developed to measure the mass of the top quark. Using eight events which are consistent with the hypothesis $t \bar{t} \rightarrow b W^{+}, \bar{b} W^{-}$ $\rightarrow b l^{+} \nu_{l}, b l^{-} \bar{\nu}_{l},(l=c, \mu)$, the mass of the top quark has becn measurcd to be $153.5_{-14.9}^{+17.1}$ (stat.) \pm 6.6 (syst.) GeV. A measurement of $155.4{ }_{-23.6}^{+27.1}$ (stat.) \pm 6.9 (syst.) $\mathrm{GeV}$ is obtained using the five events which are consistent with the $t \bar{t} \rightarrow$ $b W^{+}, \bar{b} W^{-} \rightarrow b e^{+} \nu_{e}, \bar{b} e^{-} \nu_{e}$ decay. No candidate cvents were observed which are consistent with the $t \bar{t} \rightarrow b W^{+}, \bar{b} W^{-} \rightarrow b \mu^{+} \nu_{\mu}, \bar{b} \mu^{-} \bar{\nu}_{\mu}$ decay. The combination of the two independent measurements yicld a measurement of

$$
151.1_{-12.8}^{+14.2} \text { (stat.) } \pm 6.6 \text { (syst.) GoV. }
$$

This is the first measurement of the mass of the top quark in the di-lepton channcls from nearly $230 \mathrm{pb}^{-1}$ of $p \bar{p}$ of collider data collected in Run II using the DØ detector.

In the immediate future, with the inclusion of more recent data, collected from spring 2004 until summer 2004, the statistics is expected to nearly double. This will lead to a more precise measurement of the mass of the top quark in the di-lepton channels. Double the data set will be useful, since the bias that arises potentially due 
to small statistics will be anmulled. While the statistical uncertainty will improve due to the increased data size, efforts are under way to further reduce the uncertainties due to systcmatic ellects as well. The Ievatron program will continue to doninate the proceedings in collider physics. It is the aim of the Tevatron program to measure the mass of the top quark precisely, up to an uncertainty of about $2 \mathrm{GeV}[96]$.

The Large Hadron Collider (LHC) program at CERN is expected to begin in the next couple of years. With nearly seven-fold increase in the center of mass energy and higher luminosity, the lacility is expected to produce top quark events much more frequently. That is why it is dubbed to be the first "top factory". While the main thrust of the LIIC program is to discover physics which is not described by the Standard Model, the current measurements related to the top quark will reach toward precision measurement. It is projected that the LIIC data will reduce the uncertainty on the measured mass of the top quark to about 1 to $2 \mathrm{GcV}[97]$. Bcfore concluding this chapter, it is worthy to quote from the August 2004 issue of Physics Today, [pages 26-27] "Re-cvaluation of Top Quark Data Raiscs Estimate of Higgs Boson's Mass":

"But, theorists contend a further tenfold reduction in the uncertainty is necessary for full exploitation of what LHC will have learned about the Higgs. Such spectacular precision, however, will require the $500 \mathrm{GeV}$ electron-positron linear collider that's at the top of the particle physics community's wish list". 


\section{Appendix A}

\section{Glossary}

A glossary of terminology used in this disscrtation is obtainod from Bock[98].

\begin{tabular}{ll}
\hline term & explanation \\
\hline calorimeter & A composite detector using total absorption of \\
& particles to mossurc the cncrgy and position of \\
incident particles and jets.
\end{tabular}




\begin{tabular}{|c|c|}
\hline term & explanation \\
\hline jet & $\begin{array}{l}\text { Products of the fragmentation of a quark into a } \\
\text { collimated group of particles that are emitted along } \\
\text { the quark's original direction. }\end{array}$ \\
\hline luminosity & A measure of the intensity of colliding beam machines. \\
\hline pilcup & $\begin{array}{l}\text { Background signals which add to obscrved cvents, } \\
\text { originating in multiple cvents that occur in the } \\
\text { same time gate as signal of interest. At the Tevatron's } \\
\text { luminosity multiple collisions may occur during a bunch } \\
\text { crossing, giving rise to such events. }\end{array}$ \\
\hline $\begin{array}{l}\text { radiation } \\
\text { damage }\end{array}$ & $\begin{array}{l}\text { (In the context of semiconductor detectors) it is the } \\
\text { general alteration of the operational and detection } \\
\text { propertics of a detector duc to high doses of irradiation. } \\
\text { In scmi-conductor devices, high-cnergy particles produce } \\
\text { three main types of effects: dislocation of atoms from } \\
\text { their nominal lattice site, transient ionization and } \\
\text { long term ionization. }\end{array}$ \\
\hline sphericity & $\begin{array}{l}=\frac{3}{2} \min \left(\sum \vec{p}_{T}^{2} / \sum \vec{p}^{2}\right) \\
\text { where, } p_{T} \text { is the transverse momentum perpendicular } \\
\text { to a unit vector } \hat{n} \text {, the sums are over all particles of the } \\
\text { reaction, and the minimum is lonmed with respect to } \hat{n} \text {. }\end{array}$ \\
\hline trigger & $\begin{array}{l}\text { A combination of electronics and informatics providing a } \\
\text { fast signal whenever some interesting event has happened. }\end{array}$ \\
\hline
\end{tabular}




\section{Appendix B}

\section{A brief history of my efforts}

This scetion deals with a varicty of tasks preformed while learning the ropes at the $D \emptyset$ experiment.

As a service task to the hardware clforts for the experimont, I worked as part of the Silicon Track Trigger team[99]. With guidance from Eric Hazen as well as Ulrich, I designed and implemented a software package[100] with could diagnose nearly 50 features related to the functionality of two daughter cards: the Link Transmitter Board and the Link Receiver Board. The entire set of tests were conducted within three minutes. The package was then used to test over 100 boards.

In order to get hands-on experience with event simulation, an event generator was designed and developed for studying event, kincmatics at a preliminary level. A two-body decay computed separately in two stages was implemented to mimic a simplistic model of the decay of the top quark. Event kinematics obtained from this 'home-made' cvent generator was compared to distributions obtained from Pythia. Furthermore, simple studies were done to enhance the production of simulated background Monte Carlo process. This is illustrated in Appendix D.

Lsing a trial and error approach, a preliminary event selection was obtained for simulated events. This event selection was then used to present the first results 
of my analysis algorithm at the Amcrican Physical Socicty April 2002 conlerence. However, a more optimal approach has been laid out by the team obtaining the cross-section measurement of the signal process related to this disscrtation. As a service task, I analyzed the data from the precision readout from the central electromagnetic trigger towers from late November 2001 until spring 2002. These efforts are described in Appendix E.

My efforts in Chapter 7 were restricted to analysis of jets. I was involved in obtaining the showering corrections of jets from November 2002 to summer $2003^{1}$. In spring 2003 average corrections were obtained for the 4-vectors of jets to represent the 4 -vectors. Both corrections were used in analysis presented during summer 2003.

The Run I analysis software from I)r. Heintz was used to obtain the mass estimator used in the analysis. IIowever, the software was dependent on other Run I software, imput and output tools. The software was made framework independent and used for this dissertation. The design and its basic implementation of the analysis soltware for this disscrtation was done within a weck for the American Physical Society's April 2002 conference. However, numerous improvements and related functionality have been added since then.

\footnotetext{
${ }^{1}$ During this period, the output format of data changed, and considerable eflort went toward implementing the necessary software to analyze data.
} 


\section{Appendix C}

\section{Interactions of final-state particles in the detector}

Collider detectors envelope the nominal interaction point. The final-state products interact with various detector sub-systems to leave characteristic signatures of their interaction. The tracking detectors measure the particle's position as a liunction of time with minimal energy loss. The calorimeter measures its energy with no time resolution. High cnergy clcctron, photon, mon, hadron, and ncutrino interaction with the detector material is relevant to this thesis, and this section briefly describes their interactions ${ }^{1}$.

Flectrons with energy greater than $100 \mathrm{MeV}$ primarily lose energy via bremsstrahlung. In this process, the emitted photon carries off a large fraction of the electron's initial cnergy. For photons with cnergy grcatcr than $\sim 100 \mathrm{McV}$, pair production is the dominant mode of energy loss. This gives rise to electron positron pairs, which in turn lose energy as described.

A single electron or photon can develop into an electromagnetic shower, consisting of many electrons and photons. The shower continues to develop until the energy of the danghter particles fall below $100 \mathrm{McV}$, at which point the mechanism of encrgy

\footnotetext{
${ }^{1}$ This generic information involves particle as well as their anti-particles. The anti-particles are not exclusively addressed here.
} 
loss becomes ionization and excitation of atomic clectrons.

Hadronic particles interact to yield showers in the bulk of the hadronic calorimeter. About half the incident hadron energy is passed on to additional sccondarics via inelastic scattering. These secondaries have more transverse energy than those produced via electromagnetic interactions (EM showers). Therefore, the hadronic showers have a larger transverse spread than the EM showers[101]. The rest of the energy is lost in the production of multiple slow pions and nucleons.

High energy muons lose energy primarily via ionization of matter in the detectors. Interaction via bremsstrahlung is at a much slower rate compared to the electrons because the muon mass is nearly two hundred times that of the electron.

Neutrinos do not interact with the detector at all. Those having a large transverse momentum leave a large imbalance in momentum along the transverse direction.

A detailed description of particle interaction is beyond the scope of this disscrtation. References [101], [102], [103] and [104] provide additional reading material for more information on this subject. 


\section{Appendix D}

\section{An illustration of the application of simple topological criteria towards optimizing the Monte Carlo production}

This section represents a simple application of topological criteria to extract optimal number of background events ${ }^{1}$.

While analyzing $t \bar{t}$ decays to di-lepton final states, one inevitably comes across background events. The signal process in the di-electron channel

$$
p \bar{p} \rightarrow t t+X \rightarrow e^{+} e^{-} b \bar{b} \bar{\nu}_{\epsilon} \nu_{e}+X
$$

will be dominated by background from the

$$
p \bar{p} \rightarrow \gamma^{*} / Z+X \rightarrow e^{+} e^{-}+X
$$

process. Similarly, the di-rmuon events

$$
p \bar{p} \rightarrow t \bar{t}+X \rightarrow \mu^{+} \mu^{-} b \bar{b} \overline{\nu_{\mu}} \nu_{\mu}+X,
$$

\footnotetext{
${ }^{1}$ This task was accomplished and documented in October, 2001.
} 
will be laked by

$$
p \bar{p} \rightarrow \gamma^{*} / Z+X \rightarrow \mu^{+} \mu^{-}+X
$$

One can also expect that

$$
p \bar{p} \rightarrow \gamma^{*} / Z+X \rightarrow \tau^{+} \tau^{-}+X,
$$

with the $\tau$ lepton decaying leptonically, or hadronically, will be a potential candidate for faking the signal cvents.

Let us take a closer look at the di-electron channel ${ }^{2}$. The signal event and the fake event have at least a pair of high $p_{T}$ electrons in the final state. While the former process has at, least, two high $p_{T}$ jets $^{3}$ the latter process is less likely to give rise to a pair of jets. This section deals with the study of the latter type of events, the $Z \rightarrow e^{+} e^{-}$background events.

Consider Table D.1, which projects the expected number of signal and background events produced in $2 \mathrm{fb}^{-1}$. It would be beneficial to study a hundred times more signal events than we actually expect alter selection cuts, from collider data. Study of a larger number of events will reduce statistical fluctuations by nearly ten times. Wo expect ${ }^{4}$ nearly 50 events after our signal cuts are applied, thus wo use about 5000 signal $t \bar{t} \rightarrow e^{+} e^{-} X$ events. We need to study a proportionate number of the background events as well. That would imply processing nearly 20 million $Z$ $\rightarrow e^{+} e^{-}$cvents. This task would be very cpu intensive. Since a small fracton of the $Z \rightarrow e^{+} e^{-}$events have two or more high $p_{T}$ reconstructed jets, only a small fraction

\footnotetext{
${ }^{2}$ For the sake of simplicity we now consider only di-electron events. The general arguments can be applied for the di-muon as well as the $e \mu$ events.

${ }^{3}$ These come from the hadronization of the $b$ quark.

${ }^{4}$ These estimations were based on a preliminary cstimation in summer 2001.
} 
of the total events produced will be able to lake our signal events.

\begin{tabular}{l|r|r|r|r}
\hline Process & $\sigma(\sqrt{s}=1.96 \mathrm{TcV})$ & $\begin{array}{r}\text { assumed } \\
\text { Branching } \\
\text { fraction } \\
\text { (in \%) }\end{array}$ & $\begin{array}{r}\text { Projected } \\
\text { \# events } \\
\text { produced } \\
\text { in Run II } \\
\left(2 \mathrm{fb}^{-1}\right)\end{array}$ & $\begin{array}{r}\text { Projected } \\
\text { \# events } \\
\text { selected } \\
\text { in Run II } \\
\left(2 \mathrm{fb}^{-1}\right)\end{array}$ \\
\hline$t \bar{t} \rightarrow$ all & $5.5 \mathrm{pb}$ & 100.0 & $\sim 10^{4}$ & $\sim 5 \times 10^{3}$ \\
$t \bar{t} \rightarrow e^{+} e^{-} X$ & $5.5 \mathrm{pb}$ & 1.25 & $\sim 10^{2}$ & $\sim 5 \times 10^{1}$ \\
$Z \rightarrow e^{+} e^{-}$ & $200 \mathrm{pb}$ & 100.0 & $\sim 1 \times 10^{5}$ & $\sim 2 \times 10^{5}$ \\
\hline
\end{tabular}

Table D.1: Table projecting the expected number of signal and background events in Run II. These projections were made in early 2001.

Gencration of Monte Carlo simulated events is a long drawn process. At first we use the Monte Carlo generators, like Alpgen, Pythia, Herwig, or Isajet. The output is then fed into a simulated detector (DØgstar followed by DØsim). Finally we process these using $\mathrm{D} \emptyset \mathrm{reco}$, and obtain reconstructed objects. Simulating the last two processes take much more time than the first step. Therefore, it is much more cfficient to apply certain topological cuts at the parton gencrator level (first stcp), even before the events are reconstructed. This gets rid of the bulk of events which will surely not pass the topological selection criteria on the reconstructed objects.

We now try to determine the appropriate selection critcria on the Monte Carlo events, such that the $Z \rightarrow e^{+} e^{-}$events, which are not likely to fake $t \bar{t} \rightarrow e^{+} e^{-} X$ events, can be eliminated before the reconstruction process. IIowever, we do not want potential background events to be eliminated. This study does not use the information of the signal topology at all. Moreover, at this stage detector resolution is absent. Hence, the set of critcria that will be determined will not be optimal, but rather loose. 
There are three principal, but simultancous ways we can use to reject the sample of fake events. They are:

- Jet multiplicity of the event (for all background events).

- Missing $p_{T}$ of the event (for all background events).

- The invariant mass of the two highest $p_{T^{\prime}}$ electrons (only for $Z \rightarrow e^{+} e^{-}$).

In our studies, we use a sample of ncarly 2000 inclusive $Z / \gamma^{*} \rightarrow e^{+} e^{-}$cvents overlaid with 2.5 minimum bias events. (These events were processed with the $p 8.11$ version of the standard $D \emptyset$ reconstruction software available during summer 2001.) The aim, as mentioncd beforc, is to apply some loose cuts to climinate those $Z \rightarrow e^{+} e^{-}$events which will not likely meet our eventual signal selection cuts on reconstructed (reco) objects.

In these experiments, we categorize each reconstructed event and $\mathrm{MC}$ event in two categories. For the Class I experiment the categories are defined as:

$$
\text { reco Ilag typc }=\left\{\begin{array}{c}
0 \text { if the cvent has: } \\
\geq 2 \text { jets } \\
w / p_{T}>20.0 \mathrm{GeV} \\
w / \eta \mid<2.5 \\
1 \text { otherwise. }
\end{array}\right.
$$


and,

$$
M C \text { flag type }=\left\{\begin{array}{c}
0 \text { if the event has: } \\
\geq 2 \text { particle jets } \\
w / p_{T}>10.0 \mathrm{GeV} \\
w /|\eta|<3.0 \\
\\
1 \text { otherwise. }
\end{array}\right.
$$

Here, a reconstructed jet is a calorimeter cluster energy deposit within a simple hypothetical cone object of radius $\Delta R=0.5$ unit (JCCB object). For the $Z \rightarrow e^{+} e^{-}$ we ensure that the least possible $d R>0.1$ between these jets and each and every electron object ${ }^{5}$.

The results from the $2000 Z \rightarrow e^{+} e^{-}$events are shown in Table D.2. A similar exercise is done using a thousand $\gamma^{*} / Z \rightarrow \mu \mu$ events. These events were overlaid with 2.5 minimum bias events and processed with the standard DØ reconstructed version ${ }^{6}$. Those results are illustrated in Table D.3.

\begin{tabular}{|l|l|r|}
\hline reco flag & \multicolumn{2}{|c|}{ MC flag } \\
& type $=0$ & type $=1$ \\
\hline typc $=0$ & 24 & 21 \\
type $=1$ & 109 & 1846 \\
\hline
\end{tabular}

Table D.2: Class I experiment using the $Z \rightarrow e e$ sample.

Continuing a step further with a series of experiments, the Class II experiments

\footnotetext{
${ }^{5}$ This is not a requirement for the $Z \rightarrow \mu \mu$ sample However in the $Z \rightarrow \tau \tau$ we do make such a requirement.

${ }^{6}$ Version p08.11. was used.
} 


\begin{tabular}{|l|l|r|}
\hline reco flag & \multicolumn{2}{|c|}{ MC flag } \\
& type $=0$ & type $=1$ \\
\hline type $=0$ & 15 & 5 \\
type $=1$ & 50 & 930 \\
\hline
\end{tabular}

Table 1).3: Class I experiment using the $Z \rightarrow \mu \mu$ sample.

were performed. For these experiments, the reco flag is defined as:

$$
\text { reco flag type }=\left\{\begin{array}{c}
0 \text { if the event has: } \\
\geq 2 \text { jets } \\
w / p_{T}>20.0 \mathrm{GeV} \\
w /|\eta|<2.5 \\
\text { and } 2 \text { leptons }(e / \mu) \\
w / p_{T}>15 \mathrm{GeV}(e / \mu) \\
\mu \text { w/ }|\eta|<1.7 \\
\& . \mathrm{Idnseg}>0 \\
e \text { w }|\eta|<2.5 \\
1 \text { otherwise. }
\end{array}\right.
$$

We continue to use the similar jet reconstruction algorithmn ${ }^{7}$, and also ensure that the jet is at least away from every electron object by a $d R>0.1$ just as before. The $M C$ flag definition is the same as in Equation D.2. The results from the $Z \rightarrow e^{+} e^{-}$

\footnotetext{
${ }^{7} \Lambda$ JCCB jet object.
} 
are in Table D.4, and those from the $Z \rightarrow \mu \mu$ cvents are in Table D.5.

\begin{tabular}{|l|l|r|}
\hline reco flag & \multicolumn{2}{|c|}{ MC flag } \\
& type $=0$ & type $=1$ \\
\hline type $=0$ & 10 & 1 \\
typc $=1$ & 123 & 1866 \\
\hline
\end{tabular}

Table D.4: Class II experiment using the $2000 Z \rightarrow e e$ events.

\begin{tabular}{|l|l|r|}
\hline reco flag & \multicolumn{2}{|c|}{ MC flag } \\
& typc $=0$ & typc $=1$ \\
\hline typc $=0$ & 5 & 0 \\
type $=1$ & 60 & 935 \\
\hline
\end{tabular}

Table D.5: Class II experiment using the $1000 Z \rightarrow \mu \mu$ events.

Let us now analyze the $Z \rightarrow \tau \tau$ events. Although wo will use the same analysis technique as before, we present the results in which both the final state $\tau$ leptons decay leptonically, $\tau \rightarrow e \nu_{e} / \mu \nu_{\mu}$ (called non-hadronic cvents) scparately, from those events in which at least one $\tau$ lepton decays hadronically (called hadronic events). Our sample consists of 2400 events overlaid with 2.5 minimum bias events, and processed with the same reconstructed version as used beforc. We have 288 nonhadronic events. Of these,

78 cvents are: $Z \rightarrow \tau^{+} \tau^{-} \rightarrow \nu_{\tau} \nu_{\tau} e^{+} e^{-} \nu_{e} \nu_{e}$,

84 events are: $Z \rightarrow \tau^{+} \tau^{-} \rightarrow \nu_{\tau} \overline{\nu_{\tau}} \mu^{+} \mu^{-} \nu_{\mu} \overline{\nu_{\mu}}$,

126 events are: $Z \rightarrow \tau^{+} \tau^{-} \rightarrow \nu_{\tau} \overline{\nu_{\tau}} e^{ \pm} \mu^{\mp} \nu_{e / \mu} \bar{\nu}_{\mu / e}$.

The remaining 2112 events have at least onc $\tau$ lepton decaying hadronically.

Let us first consider the non-hadronic events. In the Class I experiment, where the reconstructed and MC flags are defined in (1) and (2), we obtain the following results as in Table 1).6. The Class II experiment results are shown in Table D.7. 


\begin{tabular}{|l|l|r|}
\hline reco flag & \multicolumn{2}{|c|}{ MC flag } \\
& type $=0$ & type = 1 \\
\hline type $=0$ & 6 & 0 \\
type $=1$ & 18 & 264 \\
\hline
\end{tabular}

Table I).6: Class I experiment using 288 non-hadronic $Z \rightarrow \tau \tau$ event sample.

\begin{tabular}{|l|l|r|}
\hline reco flag & \multicolumn{2}{|c|}{ MC flag } \\
& type $=0$ & type $=1$ \\
\hline typc $=0$ & 0 & 0 \\
type $=1$ & 24 & 264 \\
\hline
\end{tabular}

Table D.7: Class II experiment using 288 non-hadronic $Z \rightarrow \tau \tau$ event sample.

Now consider the hadronic events. The Class I experiment results are shown in Table D.8, and the Class II experiment results are in Table D.9.

'Therefore, by applying loose cuts at the MC level over $90 \%$ of background events which will surely not meet signal criteria are eliminated. At the MC level, at least two particle jets in the background event are required. Events which mect this critcria are more likely to fake the signal events, and these can be further processed incorporating the complete detector interactions. 


\begin{tabular}{|l|l|r|}
\hline reco flag & \multicolumn{2}{|c|}{ MC flag } \\
& type $=0$ & type $=1$ \\
\hline typc $=0$ & 31 & 29 \\
typc $=1$ & 284 & 1768 \\
\hline
\end{tabular}

Table D.8: Class I experiment using 2112 hadronic $Z \rightarrow \tau \tau$ event sample.

\begin{tabular}{|l|l|r|}
\hline reco flag & \multicolumn{2}{|c|}{ MC flag } \\
& type $=0$ & type $=1$ \\
\hline type $=0$ & 2 & 0 \\
type $=1$ & 313 & 1797 \\
\hline
\end{tabular}

Table D.9: Class II experiment using 2112 hadronic $Z \rightarrow \tau \tau$ event sample. 


\section{Appendix E}

\section{Some L1 TT studies}

This section outlines studies performed to determine the efficiency of the L1 'I'T's and identify defective, or hot TTs.

In cach layer, $2 \times 2$ adjacent calorimeter colls, in $(\eta, \phi)$ space arc uniquely grouped into a TT. Analogous to the cells, the TTs are also assigned unique integer $\eta$ and $\phi$ indices to designate their position. Given a particular eta index of the TT, there are 32 TTs covering the $\phi$ space. These TTs constitute an eta ring. Tables E.1 and E.2 illustrate briefly the realization of calorimeter cell's eta and phi indices into TT indicos.

\begin{tabular}{|c|c||c|c|}
\hline CAL_ieta_cal[k] & 'T' $\eta$ index & CAL_ieta_cal[k] & 'T'T $\eta$ index \\
\hline 1,2 & 1 & $-1,-2$ & -1 \\
3,4 & 2 & $-3,-4$ & -2 \\
5,6 & 3 & $-5,-6$ & -3 \\
7,8 & 4 & $-7,-8$ & -4 \\
.. &.. &.. &.. \\
\hline
\end{tabular}

Table F.1: Assignment of calorimeter cell $\eta$ indices into TT $\eta$ indices.

The TT $\eta$ index values of \pm 1 extend to the $\eta$ range of \pm 0.8 with respect to the center of the detector. This is the region of the calorimeter that was instrumented for the L1 trigger for most of the data discussed here. 


\begin{tabular}{|c|c||c|c|}
\hline CAL_iphi_cal[k] & TI $\phi$ index & CAL_iphi_cal[k] & T' $\phi$ index \\
\hline 1,2 & 1 & 5,6 & 3 \\
3,4 & 2 & 7,8 & 4 \\
.. &.. &.. &.. \\
\hline
\end{tabular}

Table F.2: Assignment of calorimeter cell $\phi$ indices into TT $\phi$ indices.

Seven innermost layers ${ }^{1}$ of the calorimeter constitute the EM calorimeter. These layers are denoted by layer indices 1-7. The TTs which lie within the central EM calorimeter are the CEM T'l's. The energy $(E)$ of all cells in a T"T are summed up to obtain the total $E$. The total $E_{T}$ is defined as $E \sin \theta$, where $\theta$ is the angle subtended between the $z$ axis of the detector, and the line through the nominal origin of the detector and the center of a calorimeter cell ${ }^{2}$.

The role of L1 readout as a diagnostic tool is illustrated here.. Comparing the number of times each TT had the highest $E_{T}$ in an event to an avcrage value, one can identify possible noisy or faulty towers. For a long run, under normal circumstances, one would expect that all T'Ts fire the same number of times, within the limits allowed by statistical fluctuations. One can easily identify the coordinates of the TTs giving statistically inconsistent counts and investigate further if they are defective or not. Figure E.3 and Figure E.1 show the spectrum of the frequency count of the maximum and the second maximum $E_{T}$ TTs respectively.

The TTs in purple and blue fire less frequently than the oncs in green, while the ones in red fire more often than the expected average. There may be a slight variation of trigger rate versus $\eta$. Ilowever, all TTs in a given $\eta$ ring should fire at the same rate. In Figure F.3 the frequency count for the TTs fired by the CEM $(1,15)$ trigger is depicted. The TTs $(-1,5),(-1,14)$ and $(-1,30)$ in purple have a very low number of

\footnotetext{
${ }^{1}$ These are the 1 em layers, however the 3rd layer is segmented into 4 finer layers.

${ }^{2}$ The cells constituting a TT will have a unique value of $\theta$.
} 

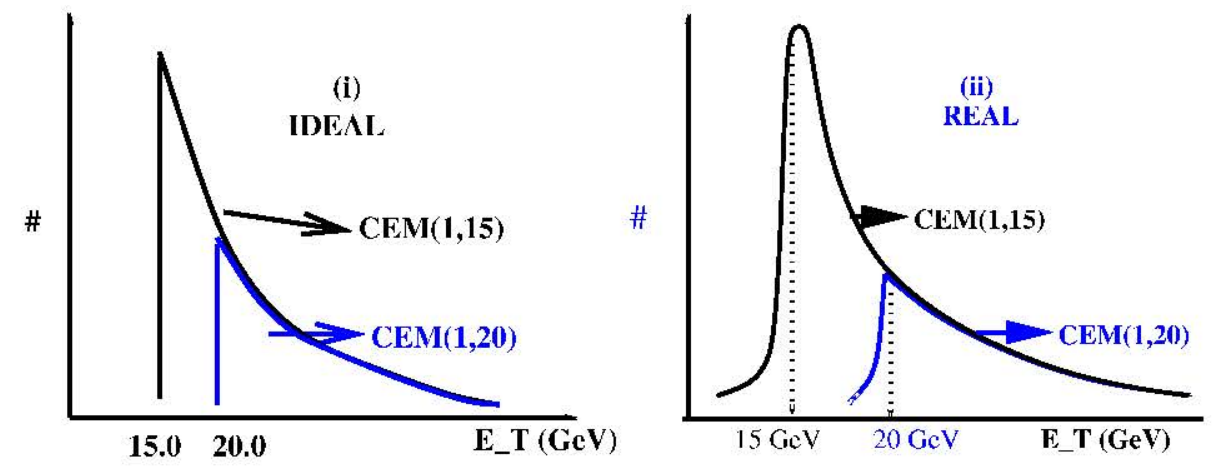

Figure E.1: Sketch showing the superposition of the $E_{T}$ spectrum. In the first plot we have the case where there is no resolution effect, as it would be in an ideal situation. In the second one we have a more realistic example.

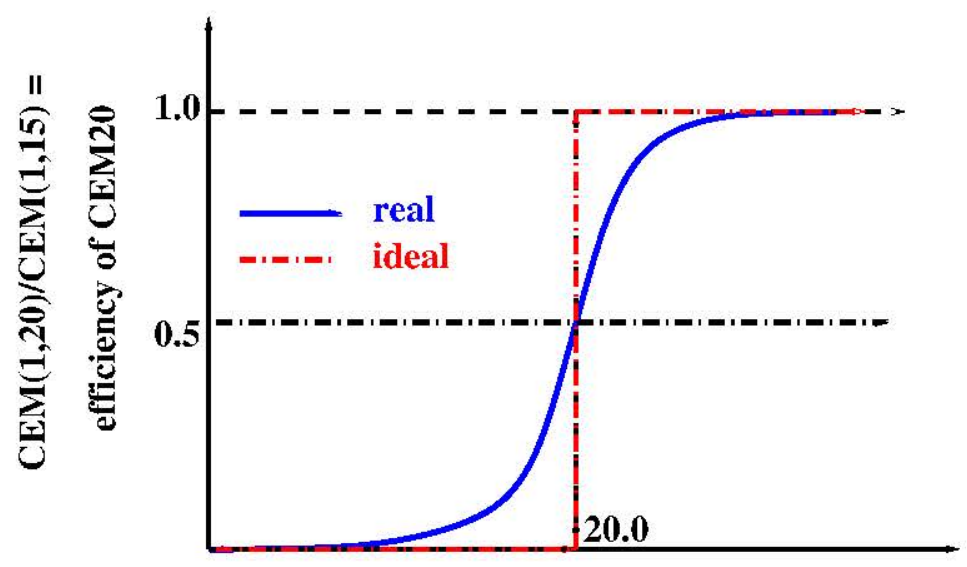

E_T of TT $(\mathrm{GeV})$

Figure E.2: Cartoon of the turn-on-curve for CEM $(1,20)$ trigger w.r.t. CEM $(1,15)$ triggcr, obtained by bin-by-bin division of histograms from Figure E.1.

cases where they are the first maximum $E_{T}$ 'T'l. Figure E.4 shows the frequency of the sccond highest TT in CEM $(2,10)$ triggers. To some extent, a correlation between the TTs response in Figure E.3 and Figure E.4 is evident. Without doubt the TTs $(-1,5),(-1,14)$ and $(-1,30)$ show a much lower count compared to the average oncs, in both cases.

In Figure E.5, a histogram of the frequency count from all 256 'T'l's is shown. As a cross check for good performance of various eta rings of the central EM calorime- 


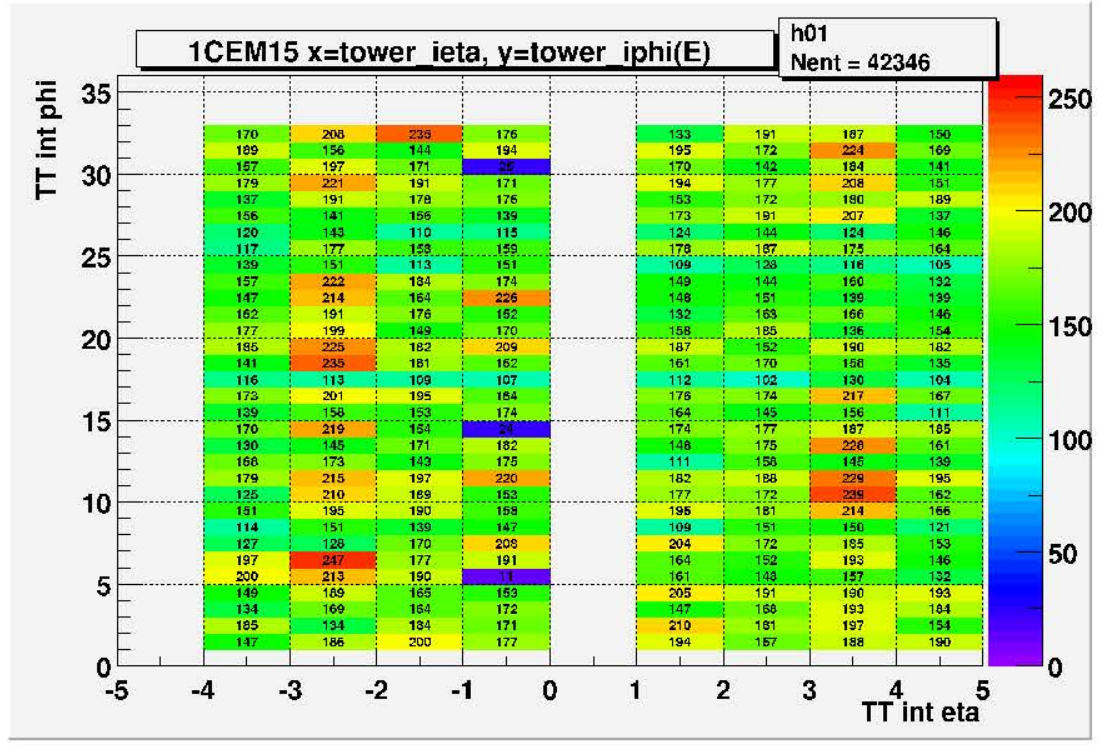

Figure F.3: Frequency with which various CEM TTs have the highest $E_{T}$ for the CEM $(1,15)$ trigger in runs 150408 and 150409.

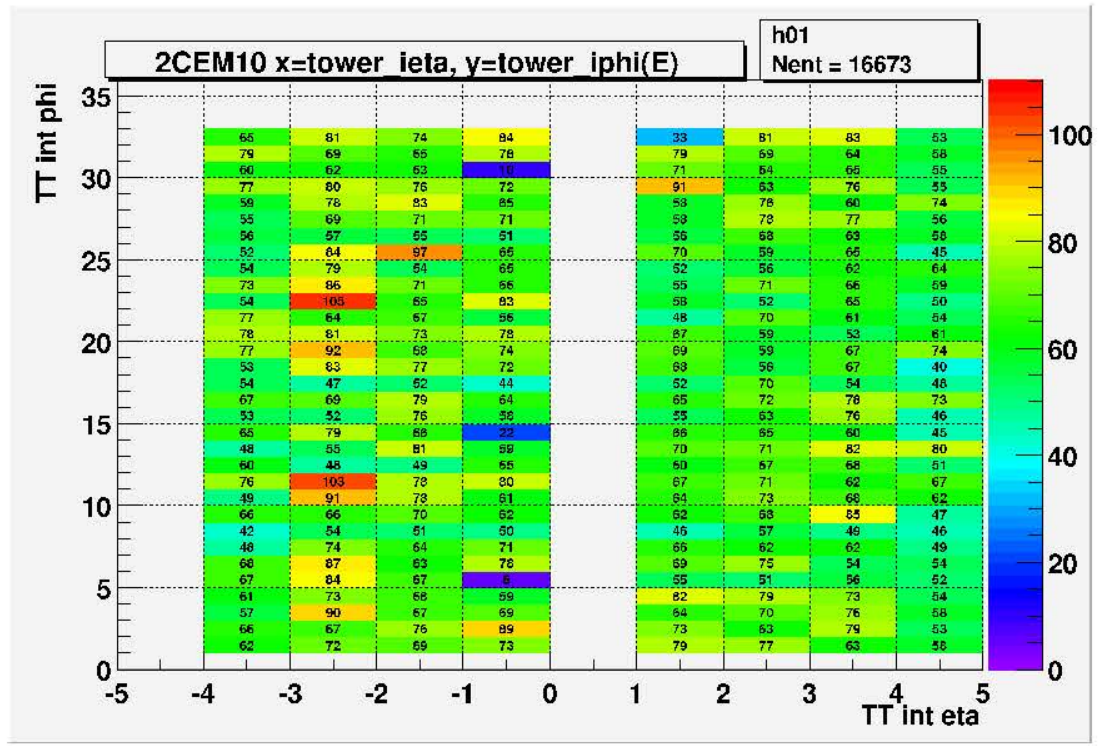

Figure E.4: Frequency with which various CEM T'T's have second highest $E_{T}$ for the CEM $(2,10)$ trigger in runs 150408 and 150409. 


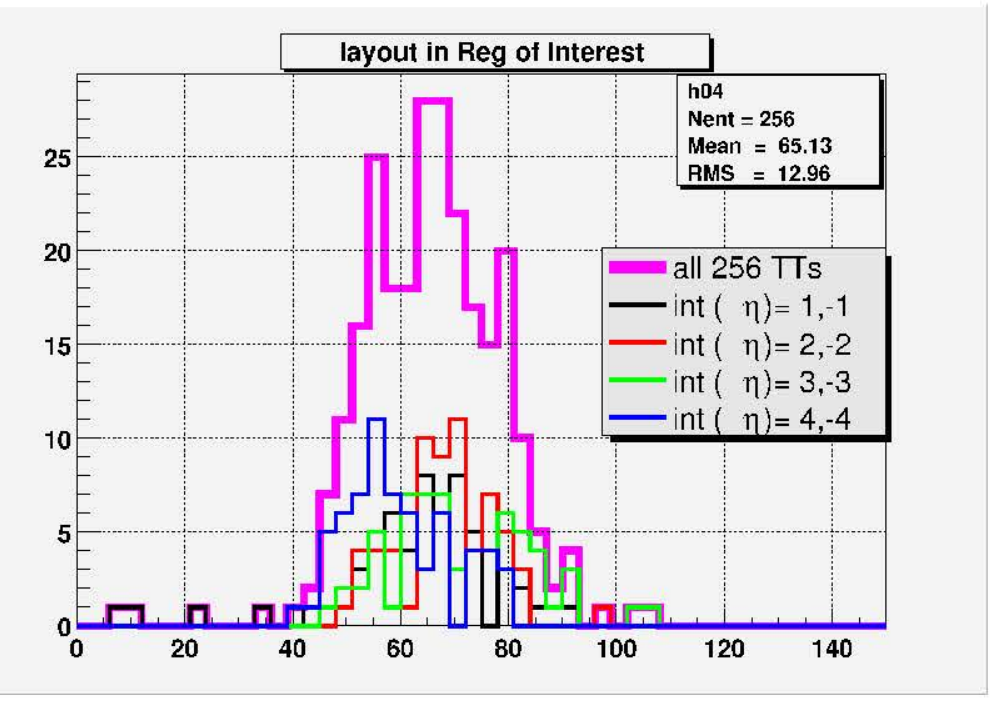

Figure E.5: Histogram of frequency counts from Figure E.4 The Mcan and RMS in the plot represent all 256 CEM T'l's. Distributions for the four inner most $|\eta|$ rings are also illustrated.

ter, one can divide the above histograms into four histograms. Each one of these corresponds to the frequency count of TTs constituted in a certain eta ring.

Another simple diagnostic is to see the $E_{T}$ spectrum of maximum TTs fired by the triggers ${ }^{3}$. Using a parent sample of CEM $(1,10)$ triggered cvents, onc can construct the CFM15 turn-on curve using CFM $(1,15)$ triggered events, as in Figure F.7. Furthermore, using di-EM triggers we can establish an unbiased measurement of the trigger efficiencies [55]. 'Therefore, as the parent sample we use the events triggered by the CEM(1,15) trigger, and construct the turn-on curve of the events fired by the di-EM CEM(2,10) trigger. Using the cvents triggered by the CEM(1,10) trigger as the parent sample we construct the turn-on curve of the events fired by the CEM(2,5) trigger. Plots for the unbiased trigger efficiency are shown in Figure E.8.

\footnotetext{
${ }^{3}$ Farly data also showed irregularities in the the low $E_{T}$ spectrum [55].
} 

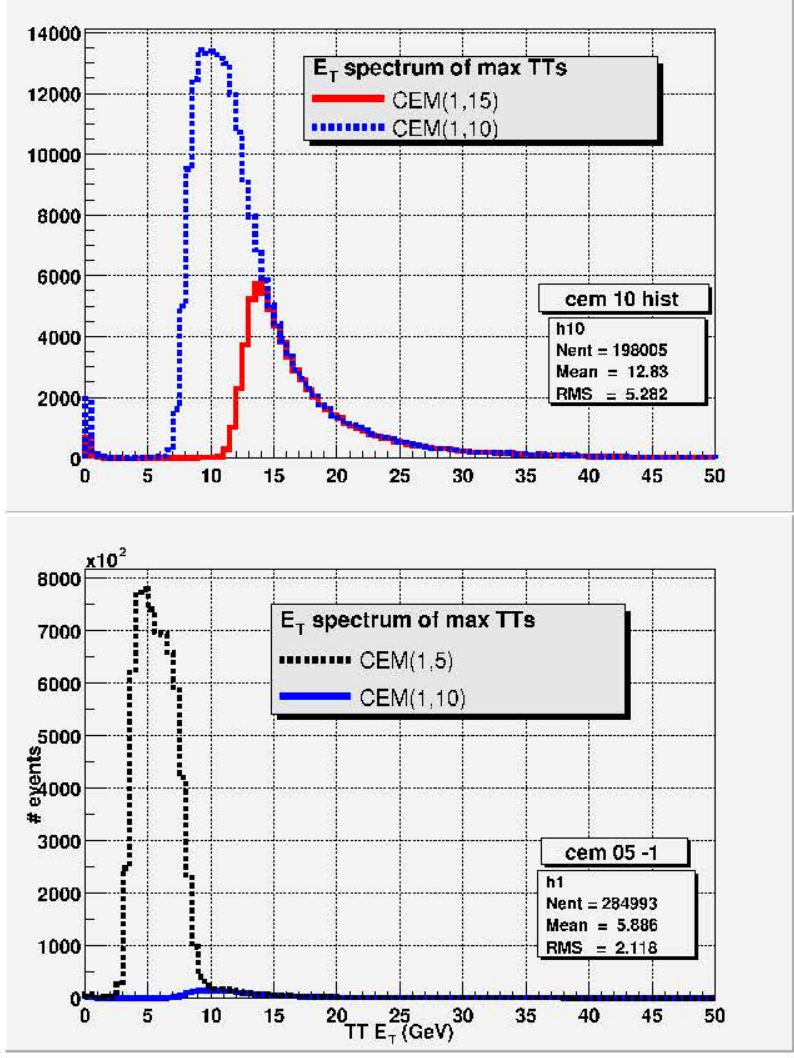

Figure E.6: Maximum $E_{T}$ spectrum of the TTs in the region of interest. The number of events triggered by $\operatorname{CEM}(1,5)$ have been appropriately corrected for prescale.

The plateau of the turn-on curves in Figure E.7 as well as Figure E.8 show a somewhat irregular profile. Defective TTs may cause the turn-on curves to show such irregularities ${ }^{4}$. Turn-on curves are established for all the 256 T'T's which are within $|\eta|<0.8$ region, where the CEM triggers are active. Some curves from individual 'T"'s are shown in Figure E.9.

From the 256 TTs, 7 werc identificd as defective[55] and their contribution was omitted from the response. The turn-on curves were again computed for the remaining TTs and are shown in Figure E.10.

\footnotetext{
${ }^{4}$ In previous analysis defective TTs have actually causod similar irregularitios.
} 


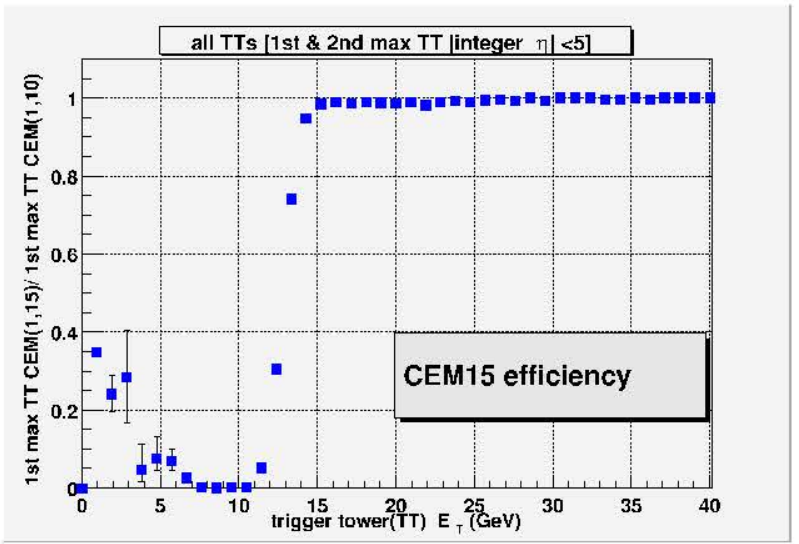

Figure E.7: The CEM15 turn-on curve. The turn-on curve for a biased measurement of the CEM15 trigger with respect to the CEM10 trigger using precision readout from the 'T'l's.

The trigger-simulator is used to create Monte-Carlo generated data. This reproduces the data from the detector as shown in Figure E.11. 

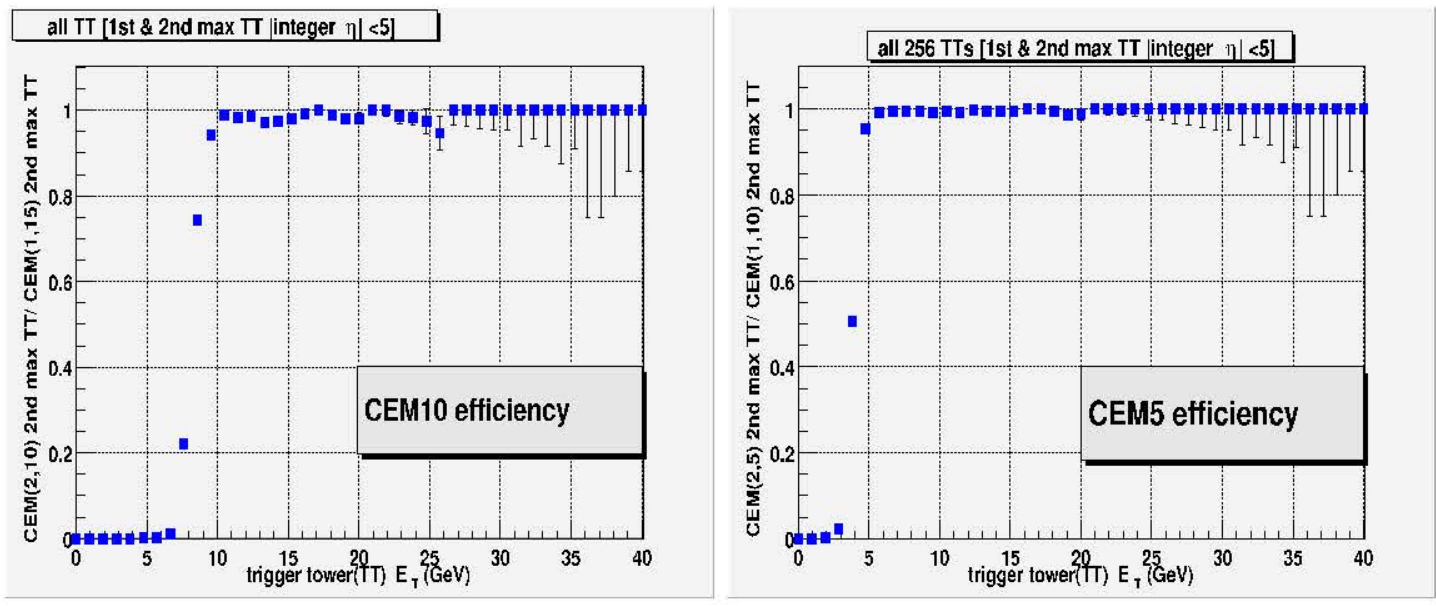

Figure E.8: The turn-on curves for some TTs in an unbiased measurement of the CEM10 and CEM5 triggers using precision readout from the TTs. 

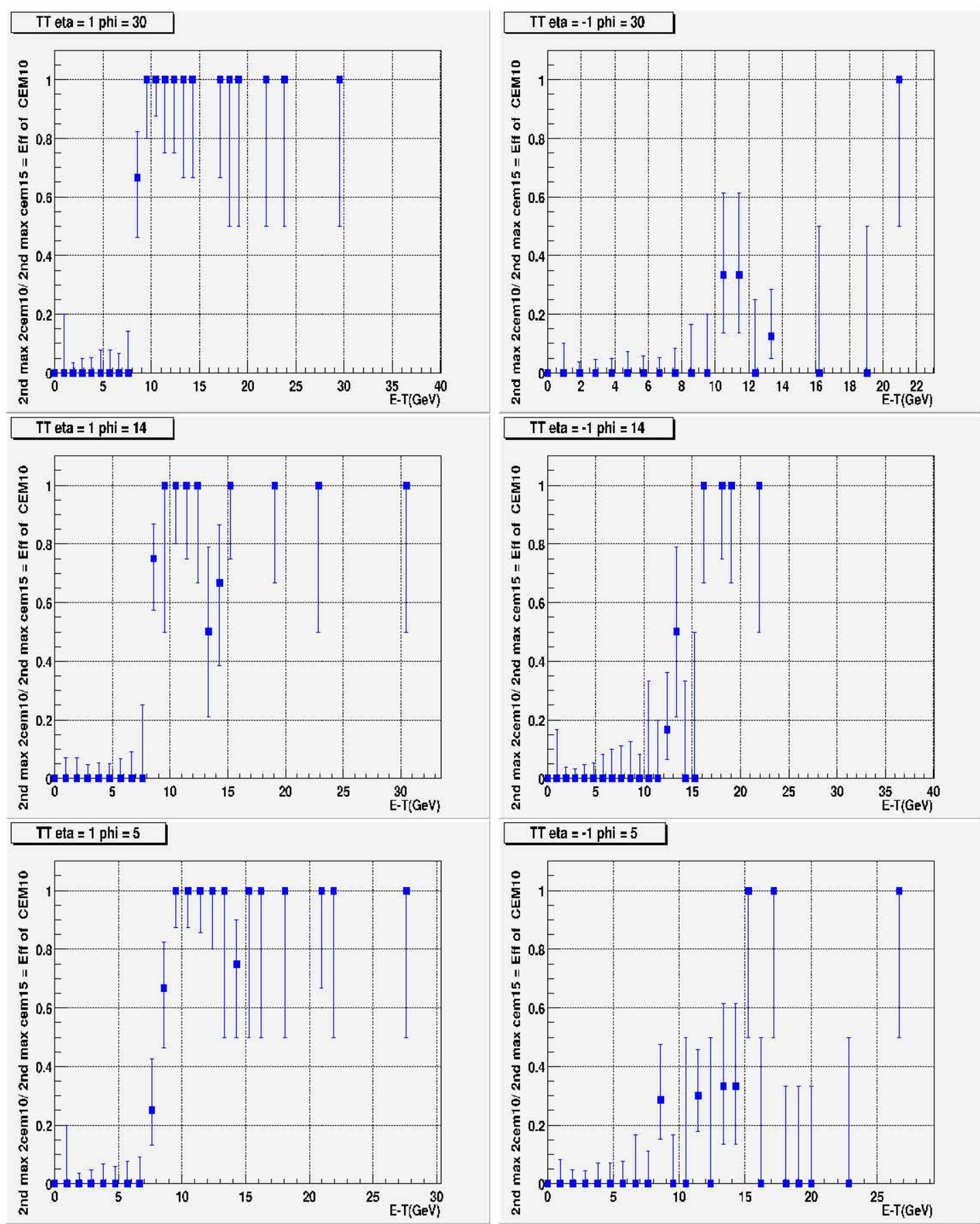

Figure E.9: The turn-on curves for some individual T'Ts. These measurements form the basis of the overall measurement in Figure E.8. The plots on the right are some of the delective T'T's in the runs. 

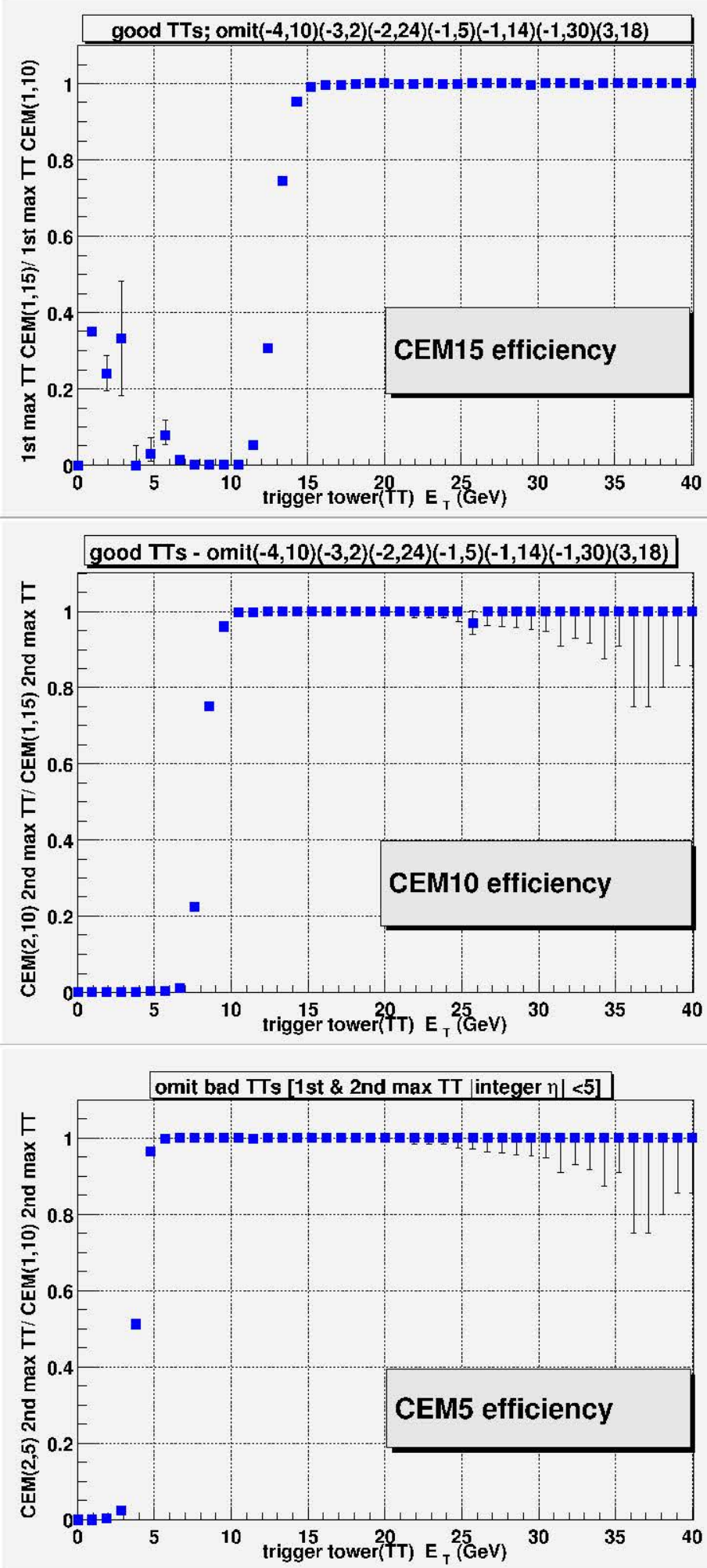

Figure E.10: The turn-on curves for CEM15, CEM10 and CEM5 triggers after the removal of delective TTs. 

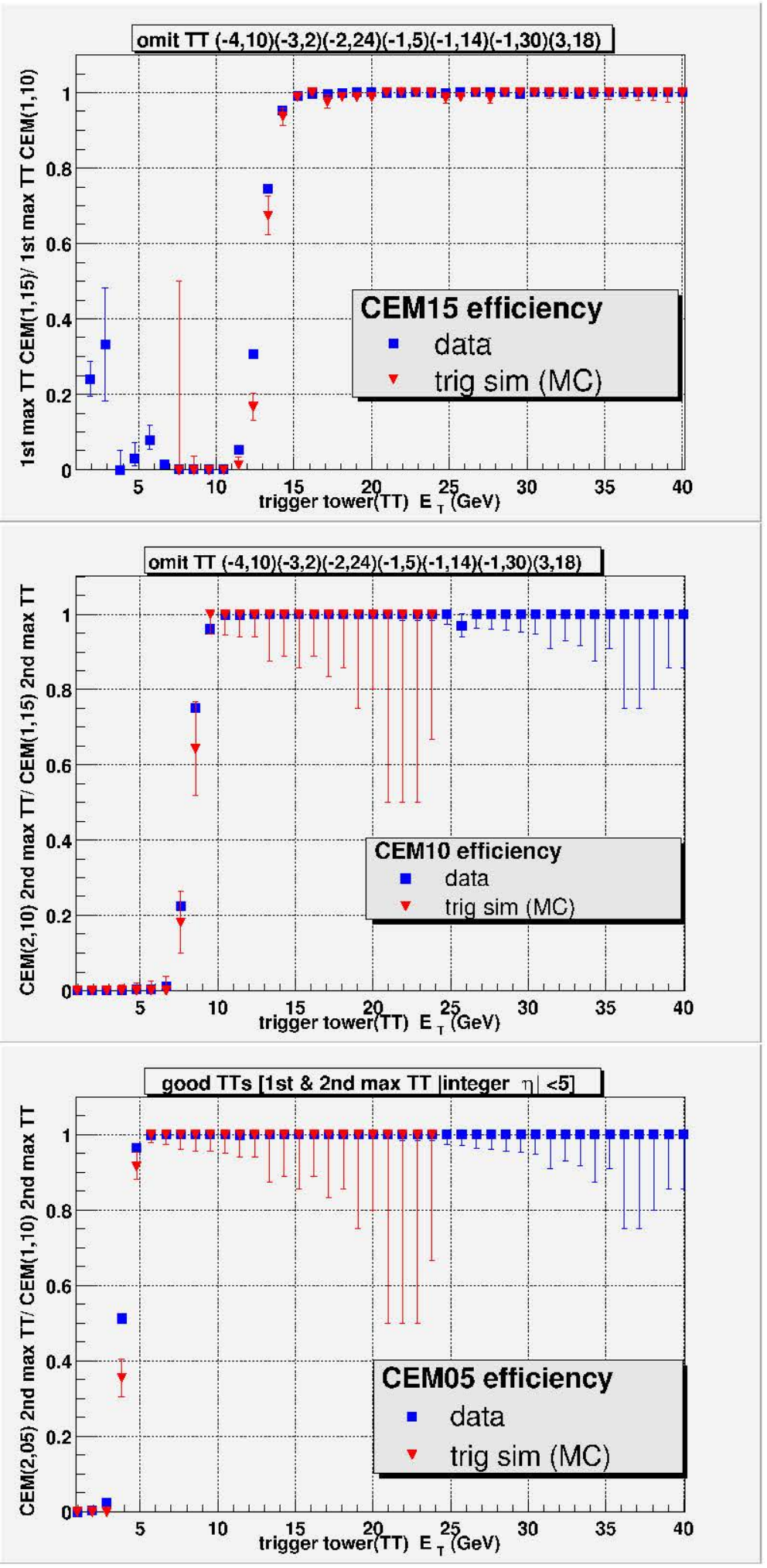

Figure E.11: The corrected turn-on curve for CEM10 and CEM5 triggers overlaid with results from the trigger-simulator. 


\section{Appendix F}

\section{Additional information regarding average corrections to the jet 4 -vectors for representing parton 4 -vectors}

For the case of jets from simple cone algorithm, the mean reconstructed energy

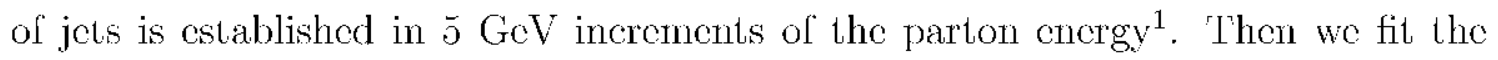
function $F_{j e t}=p_{0}+p_{1} F_{\text {parton }}+p_{2} F_{\text {parton }}^{2}$ to the mean reconstructed jet energy $\left(E_{j e t}\right)$ as a function of parton energy $\left(E_{\text {parton }}\right)$, which is illustrated in Figure 7.9 (left). Figure 7.9 (right) represents its profile (average). To extract the cnergy of a jet corrected to its parton level, we use the inverse function, obtained from solving the quadratic equation, using the solution that gives physical values of $E_{\text {parton}}$, for a. range of $F_{j e t}$ values:

$$
F_{\text {parton }}=\frac{-p_{1}+\sqrt{p_{1}^{2}-4 p_{2}\left(p_{0}-E_{j e t}\right)}}{2 p_{2}} .
$$

After obtaining the corrections, wo use them to rcconstruct the invariant mass of physical quantities of greatest interest using the same Monte Carlo events used to extract the corrections. In a series of plots we represent the reconstructed mass of

\footnotetext{
${ }^{1}$ This is established using the profile averaging functionality in the ROO'T[84] package.
} 
the $W$ boson (Figure F.1), and the mass of the $\ell$ quark (Figure F.2), alter applying the parton-level corrections to reconstructed jets. 

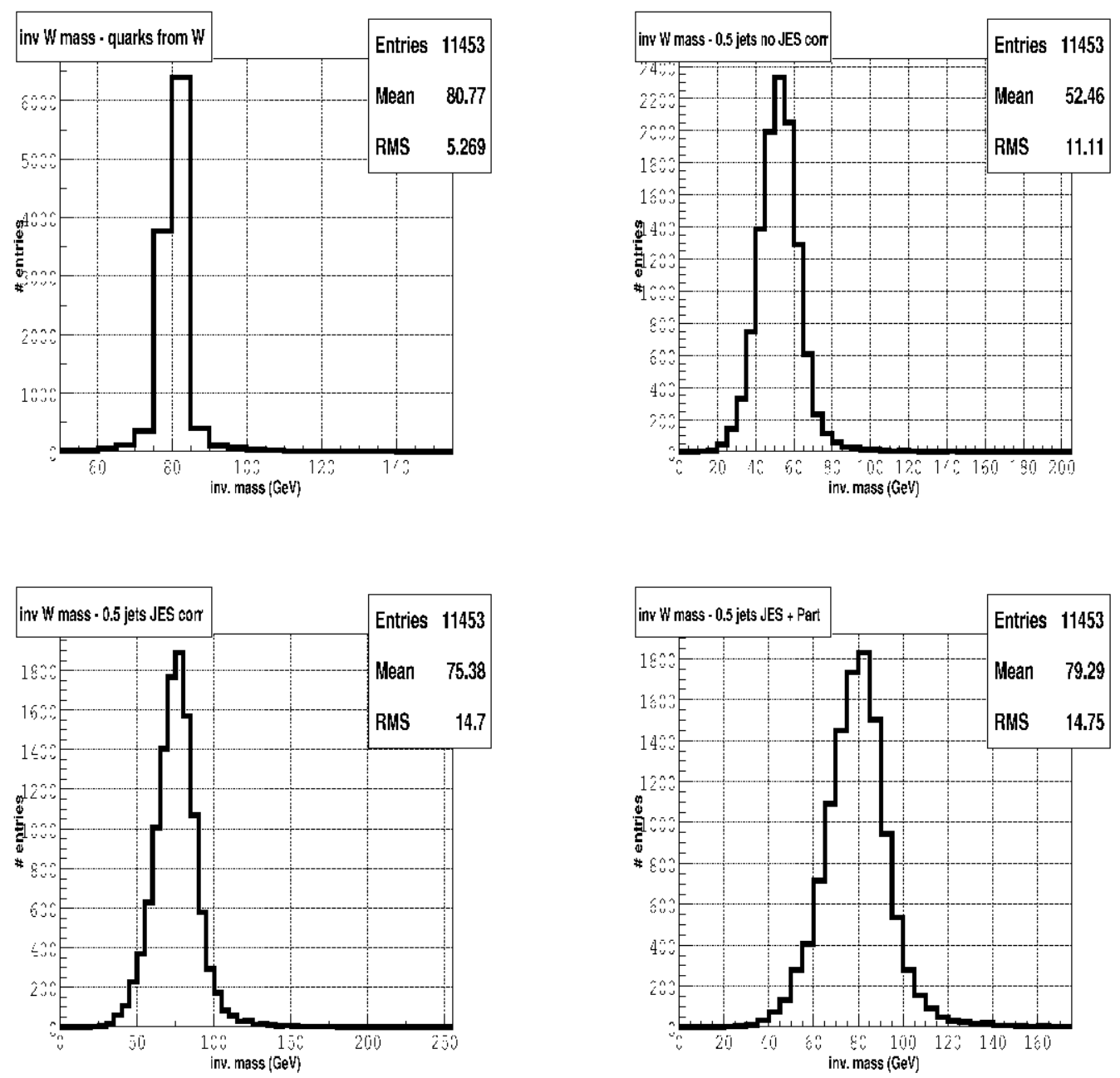

Figure F.1: The reconstructed mass of the $W$ boson from simulated events. Starting clock-wise from the upper left plot: the $W$ boson mass is reconstructed using the quarks; in the next plot the reconstructed jets are used, but without any Jet Fnergy Scale corrections $[69]$; using the energy scale corrections, as well as the parton level corrections, the $W$ is reconstructed; and in the bottom left plot the reconstructed jets arc only cncrgy scalc corrected. 

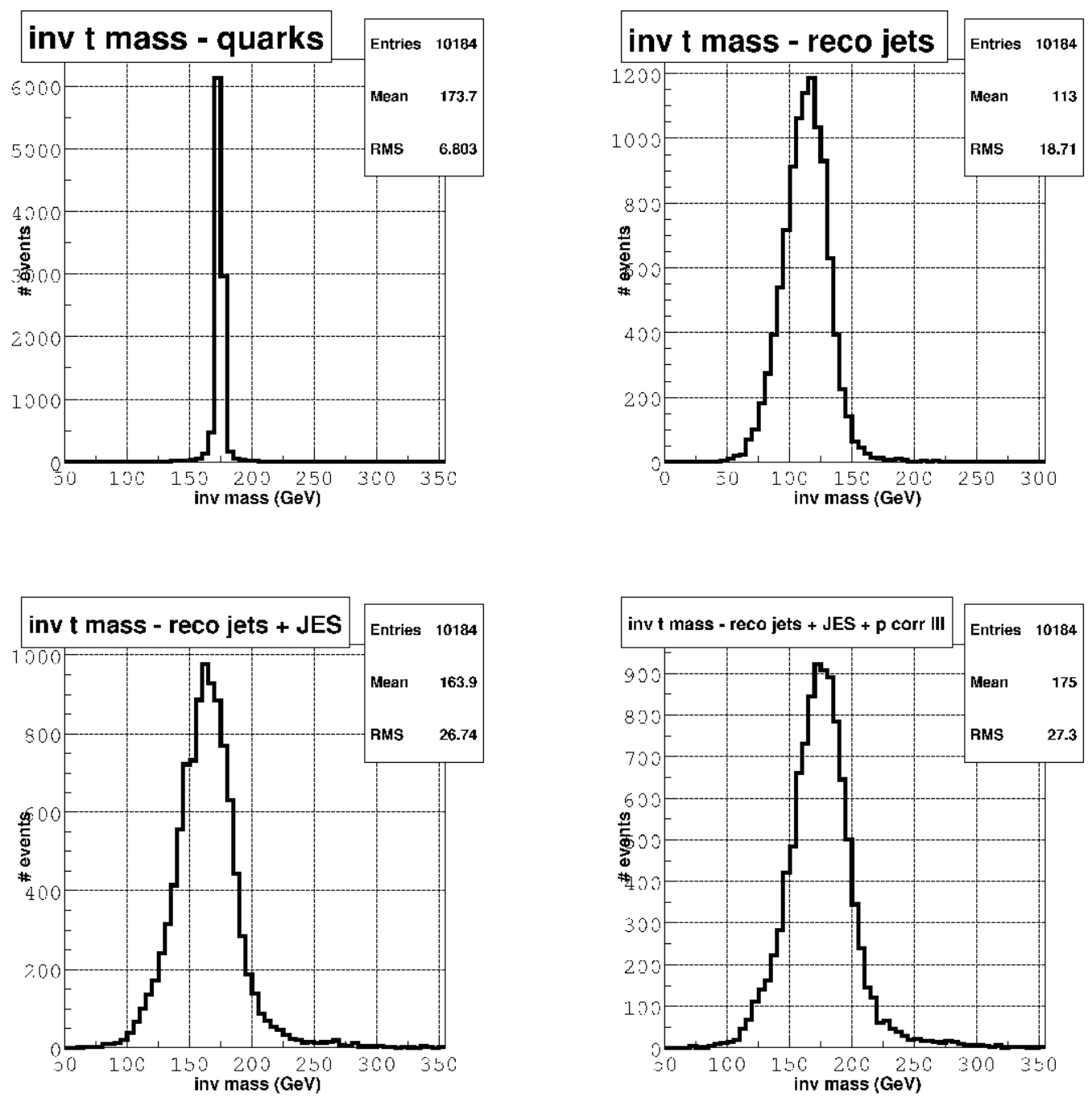

Figure F.2: Reconstructed mass of the $t$ quark from simulated events. Starting clock-wise from the upper left plot: the $t$ quark mass is reconstructed using the quarks; in the next plot the reconstructed jets are uscd, but without any Jet Encrgy Scale corrections $[69]$; using the energy scale correcitons, as well as the parton level corrections, the $t$ is reconstructed; and in the bottom left plot the reconstructed jets are only energy scale corrected. 


\section{Appendix G}

\section{Additional information regarding template distributions}

\begin{tabular}{|c|c|c|}
\hline process & $\begin{array}{l}\text { c } \mu \text { channel } \\
\# \text { entries }\end{array}$ & $\begin{array}{l}\text { ce channcl } \\
\# \text { entries }\end{array}$ \\
\hline \multicolumn{3}{|l|}{ Signal: } \\
\hline input MC $m_{t}=120 \mathrm{GeV}$ & 1033 & 590 \\
\hline input MC $m_{t}=140 \mathrm{GeV}$ & 1882 & 994 \\
\hline input $\mathrm{MC} m_{t}=160 \mathrm{GcV}$ & 1577 & 690 \\
\hline input MC $m_{t}=175 \mathrm{GcV}$ & 3675 & 1663 \\
\hline input MC $m_{t}=190 \mathrm{GcV}$ & 2231 & 1004 \\
\hline input $\mathrm{MC} m_{t}=210 \mathrm{GeV}$ & 3833 & 1774 \\
\hline input $\mathrm{MC} m_{t}=230 \mathrm{GeV}$ & 4979 & 2365 \\
\hline \multicolumn{3}{|l|}{ Physics background: } \\
\hline inclusive $Z / \gamma^{*}$ & 62 & 52 \\
\hline inclusive di-boson $\left(W^{+} W^{-}\right)$ & 157 & 61 \\
\hline \multicolumn{3}{|l|}{ Instrumental fakes: } \\
\hline$\mu+j j j$ & 42 & not applicable \\
\hline fake EMs & not applicable & 65 \\
\hline missing $E_{T}$ fakes & not applicable & 180 \\
\hline
\end{tabular}

Table G.1: Statistic of template distributions used in the analysis. These cvents are obtained after the application of event selection cuts. The selected events have $m_{\text {peak }}$ value within the range given by: $100 \mathrm{GeV}<m_{\text {peak }}<280 \mathrm{GeV}$. The initial number of events available for various templates is not the same in all cases. 
The event sclection critcria used for constructing template distributions are outlined here.

First, the criteria for the analysis in the e $\mu$ channel are highlighted.

\section{1.a Signal:}

In the $e \mu$ channel analysis, the signal events are selected with the following minimal charactcristics [52]:

1. An isolated electron matched to a track, having $p_{T}>15 \mathrm{GeV}$, within $|\eta|<1.1$ or $1.5<|\eta|<2.5$.

2. An isolated muon matched to a track, having $p_{T}>15 \mathrm{GeV}$.

3. A pair of isolated jets with $p_{T}>20 \mathrm{GeV}$.

1. The cvent missing $p_{T}>25 \mathrm{GeV}$.

5. $H_{T}=\max \left(p_{T}(e), p_{T}(\mu)\right)+\sum p_{T}(j)$, where sum is over all isolated jets with $p_{T}>15 \mathrm{GeV}$

\section{1.b Physics background:}

These are processes other than the signal process that yield a final-state resembling that of a signal process. Such events are are represented in this category. Therefore, for selecting the MC events from the physics background processes, the set of criteria in 1.a is applicd.

\section{1.c Instrumental fakes:}

In this category, an event which may have a mis-identified final-state object fakes the signal event signature. Therefore, data is used to obtain such events. For selecting the instrumental fakes from collider data ${ }^{1}$ the following criteria are applied:

\footnotetext{
${ }^{1}$ A subset of the collider data, the $K M U_{-}$extra-loose skim is used to obtain the events for the template distribution.
} 
1. An isolated EM cluster with $p_{T}>15 \mathrm{GcV}$, within $|\eta|<1.1$ or $1.5<|\eta|<2.5$.

2. An isolated muon matched to a track, with $p_{T}>15 \mathrm{GeV}$.

3. A pair of isolated jets with $p_{T}>20 \mathrm{GeV}$.

4. The event missing $p_{T}>25 \mathrm{GeV}$.

5. $H_{T}=\max \left(p_{T}(e), p_{T}(\mu)\right)+\sum p_{T}(j)$, where sum is over all isolated jets with $p_{T}>15 \mathrm{GcV}$.

Now, the ee channel cvent selcetion cirteria are outlined.

\section{2.a Signal:}

The following minimal characteristics are applicable for signal MC event selection.

1. $\Lambda$ pair of isolated clcetrons with matched tracks, having $p_{T}>15 \mathrm{GeV}$. Both clectrons are required to be within $|\eta|<1.1$ or $1.5<|\eta|<2.5$.

2. An invariant mass $\left(M_{\epsilon \epsilon}\right)$ value of the above pair not consistent with that from a $Z$ boson decay, i.e., $M_{e \epsilon}<80 \mathrm{GeV}$, or, $M_{e e}>100 \mathrm{GeV}$.

3. A pair ol isolated jets having $p_{T}>20 \mathrm{GcV}$.

4. 'The cvent missing $p_{T}>40 \mathrm{GcV}$, if $M_{e e}<80 \mathrm{GcV}$, or missing $p_{T}>35 \mathrm{GcV}$, if $M_{e e}>100 \mathrm{GcV}$.

5. The event sphericity $>0.15 \mathrm{GeV}$.

\section{2.b Physics background:}

These are processes other than the signal process that may yicld final state objects 
resembling the cvent signature. Such events are represented in this category. Therefore, for selecting the MC events from the physics background processes, criteria idcntical to that in 2.a arc applicd.

\section{2.c Instrumental fakes:}

In the ee channel, there are two sources of instrumental fakes. In the first case, if a process satisfics the cvent sclection critcria by virtuc of a mis-identified clectron (at the very least), then it is categorized as a fake EM process. The events used in the template representing instrumental EM lakes in this channel is described ${ }^{2}$. The minimal set of criteria for such an event is:

1. One electron having identical characteristics to that of the signal process describcd in 2.a (This is a probe clcctron).

2. Another electron having characteristics of the clectron described above, with the exception that it has no spatial match with a reconstructed track ${ }^{3}$. (This is the tagged electron);

3. The clectron $|\eta|$ criteria described in 2.a.

1. A pair of isolated jet objects with $p_{T}>15 \mathrm{GcV}$.

5. 'The event missing $E_{T}<10 \mathrm{GeV}$.

In the sccond case, due to detector resolution elfects of various final-state objects, the missing transverse energy may be incorrectly estimated. The primary source of such events are the inclusive $Z / \gamma^{*} \rightarrow e e+$ lake missing $E_{T}$. Dircet $Z / \gamma^{*}$ decay into a pair of electrons but no neutrinos. Such events may qualify the signal selection

\footnotetext{
${ }^{2}$ A subset of collider data, the DIEM_extra loose skim is used in this case.

${ }^{3}$ Therefore, it is devoid of the likelihood criterion as well.
} 
critcria due to missing $E_{T}$ mis-rcconstruction ${ }^{4}$. Collider data ${ }^{5}$ is used to obtain the relevant template distribution. The selection criteria used is:

1. A pair of isolated electrons with matched tracks, having $p_{T}>15 \mathrm{GeV}$. Both clectrons are required to be within $|\eta|<1.1$ or $1.5<|\eta|<2.5$.

2. An invariant mass $\left(M_{e \epsilon}\right)$ value of the above pair not consistent with that from a. $Z$ boson decay, i.e., $M_{e \epsilon}<80 \mathrm{GeV}$, or, $M_{e e}>100 \mathrm{GeV}$.

3. A pair of isolated jets having $p_{T^{\prime}}>20 \mathrm{GeV}$.

4. The event missing $p_{T}<40 \mathrm{GeV}$, if $M_{e \epsilon}<80 \mathrm{GeV}$, or missing $p_{T}<35 \mathrm{GeV}$, if $M_{e e}>100 \mathrm{GcV}$. This is opposite of the signal criterion.

5. The event sphericity $>0.15 \mathrm{GeV}$.

\footnotetext{
${ }^{4}$ More details are available from the studies by A. Kumar, et. al in the reference[52] (page 30).

${ }^{5}$ A subset of the collider data, which consists of events with at least an electron and a jet object $(e+j e t s k i m)$.
} 


\section{Appendix $\mathbf{H}$}

\section{Additional information regarding the Maximum Likelihood Estimates using the negative log-likelihood fits to event ensembles}

Numerous ensemble tests were done using ensembles with large number statistics. This was performed to ensure that there was no bias due to small statistics or oversight in the developed algorithm. It was observed that a numerical fit to the log-likelihood distributions depended on the range used by the fitting algorithm. This effect is pronounced when the ensemble size is large, (more than 100 events per ensemble).

The scrics of plots that follow represent the log-likelihood distributions of 10 distinct ensembles. Every ensemble has 500 simulated events processed without detcetor resolution effects (cvery cnscmble has a unique cvent). The numerical fits in the distributions highlight the fact that the MLEs obtained from a numerical fit over the range from $120 \mathrm{GeV}$ to $230 \mathrm{GeV}$ are different from the ones obtained from a. narrow range of $160 \mathrm{GeV}$ to $230 \mathrm{GeV}$ (the pair of input MC mass points closest to the nominal $190 \mathrm{GeV}$ point). IIere, the input value of the mass of the top quark in the signal process is $190 \mathrm{GeV}$. 
enscmble \# 0001

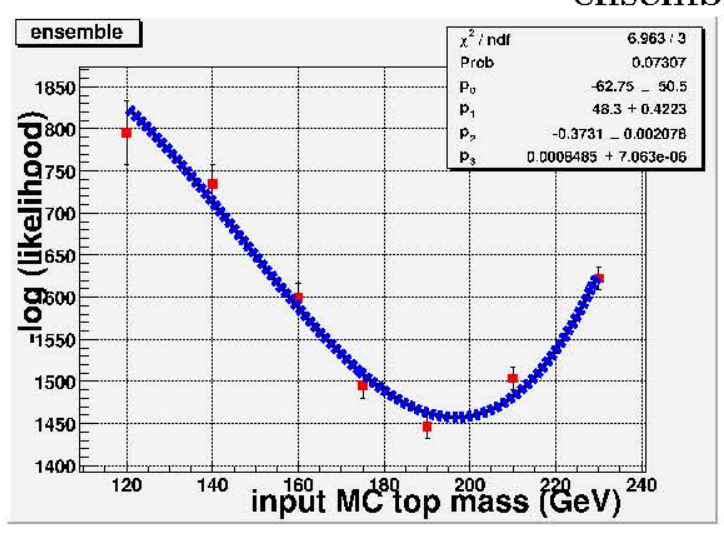

fitted $m_{t}=196.74_{+2.15}^{-2.18} \mathrm{GeV}$

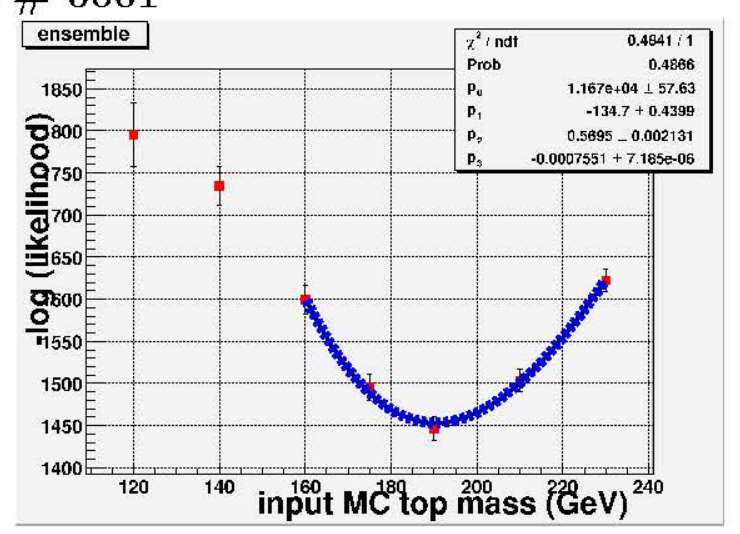

fitted $m_{t}=190.36_{+2.10}^{-2.07} \mathrm{GeV}$

ensemble \# 0002

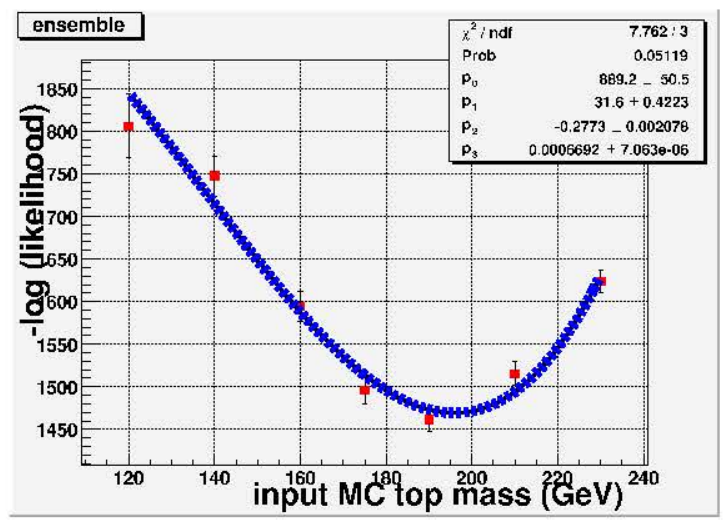

fitted $m_{t}=195.88_{+2.26}^{-2.29} \mathrm{GeV}$

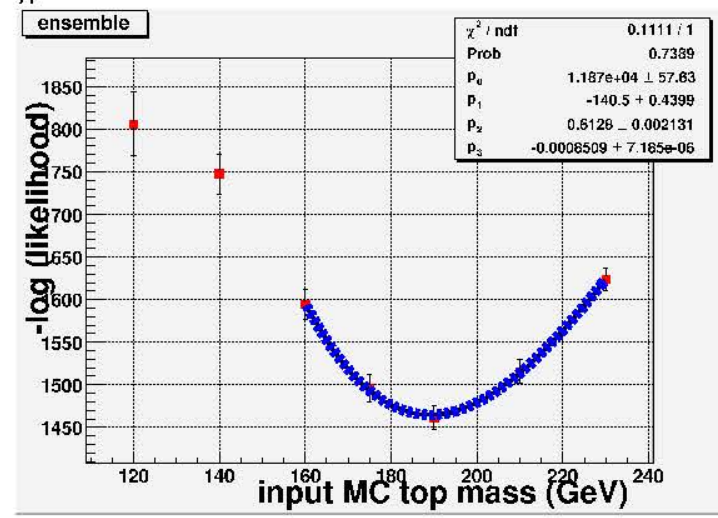

fitted $m_{t}=189.09_{+2.16}^{-2.13} \mathrm{GeV}$ 

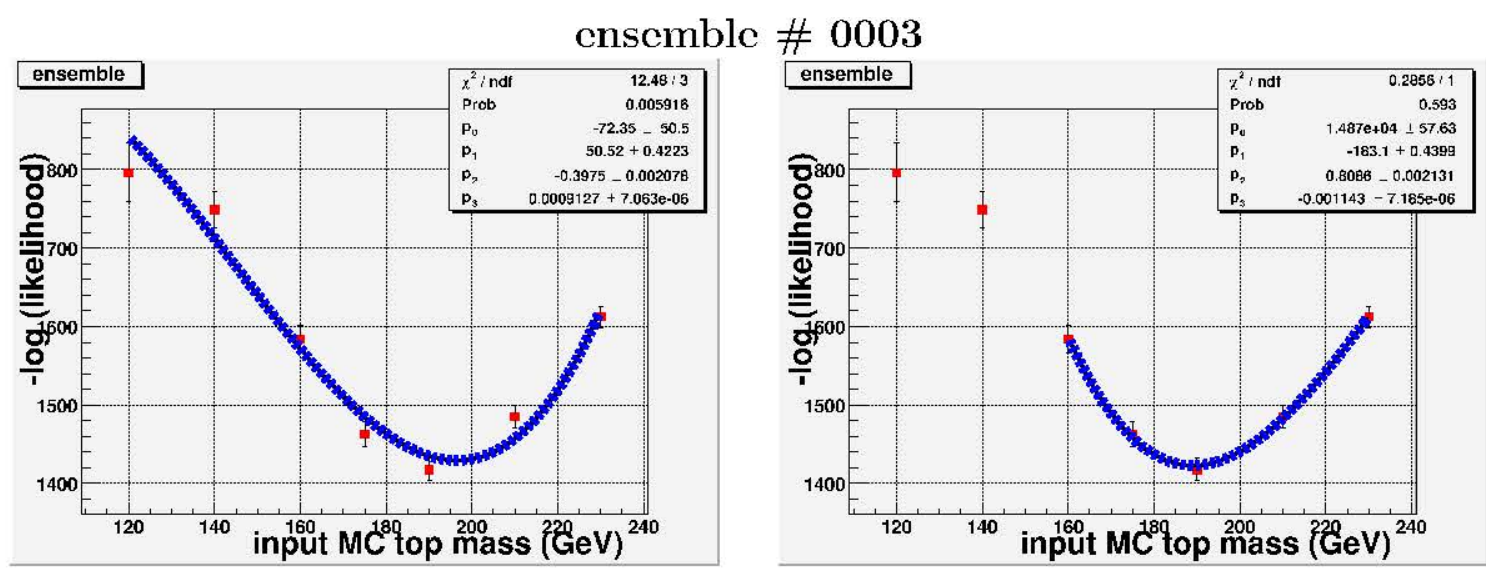

fitted $m_{t}=196.46_{+2.05}^{-2.08} \mathrm{GeV}$
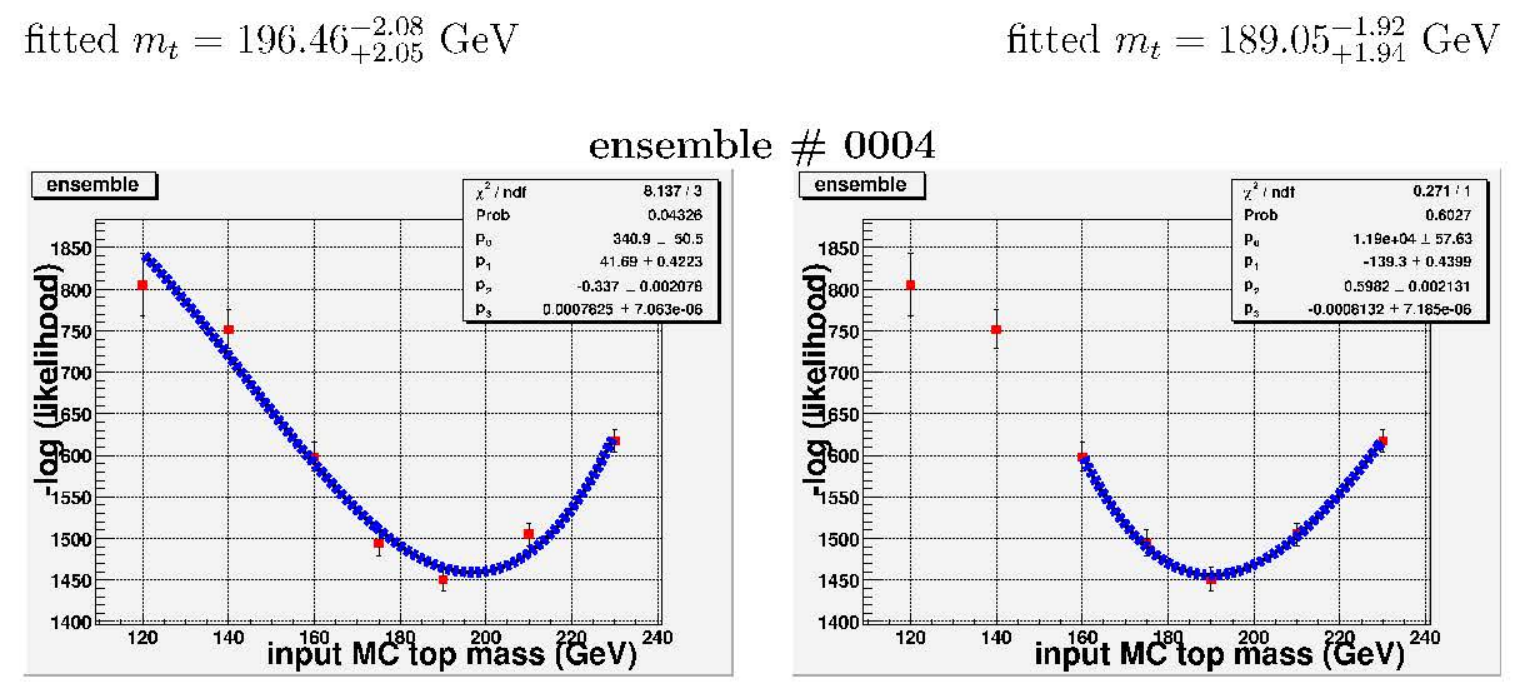

fitted $m_{t}=196.86_{+2.17}^{-2.20} \mathrm{GeV}$

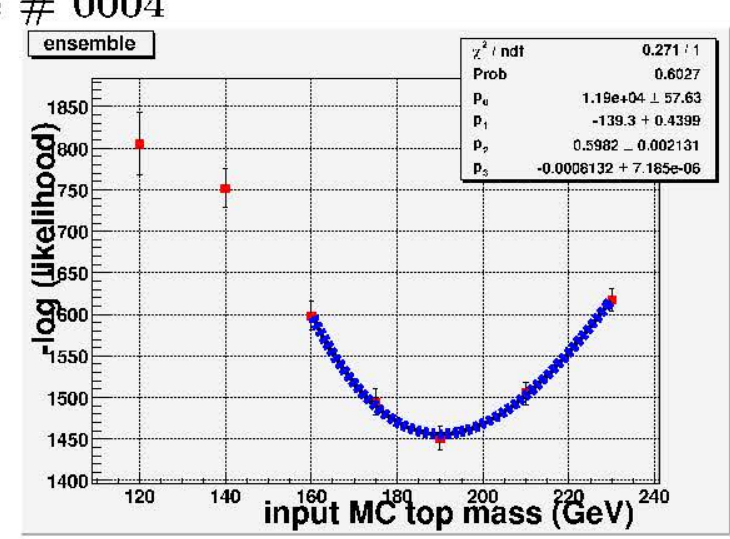

fitted $m_{t}=190.11_{+2.12}^{-2.10} \mathrm{GeV}$

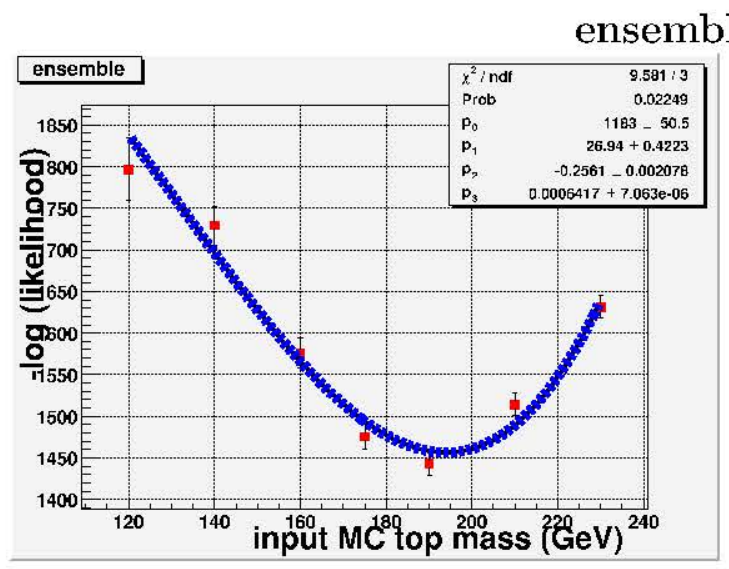

fit.ted $m_{t}=193.90_{+2.25}^{-2.27} \mathrm{GcV}$

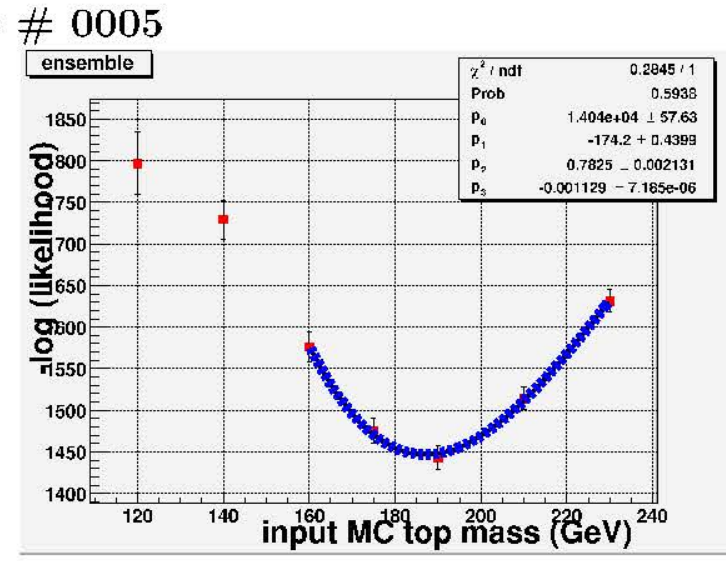

fitted $m_{t}=187.04_{+2.02}^{-1.99} \mathrm{GcV}$ 

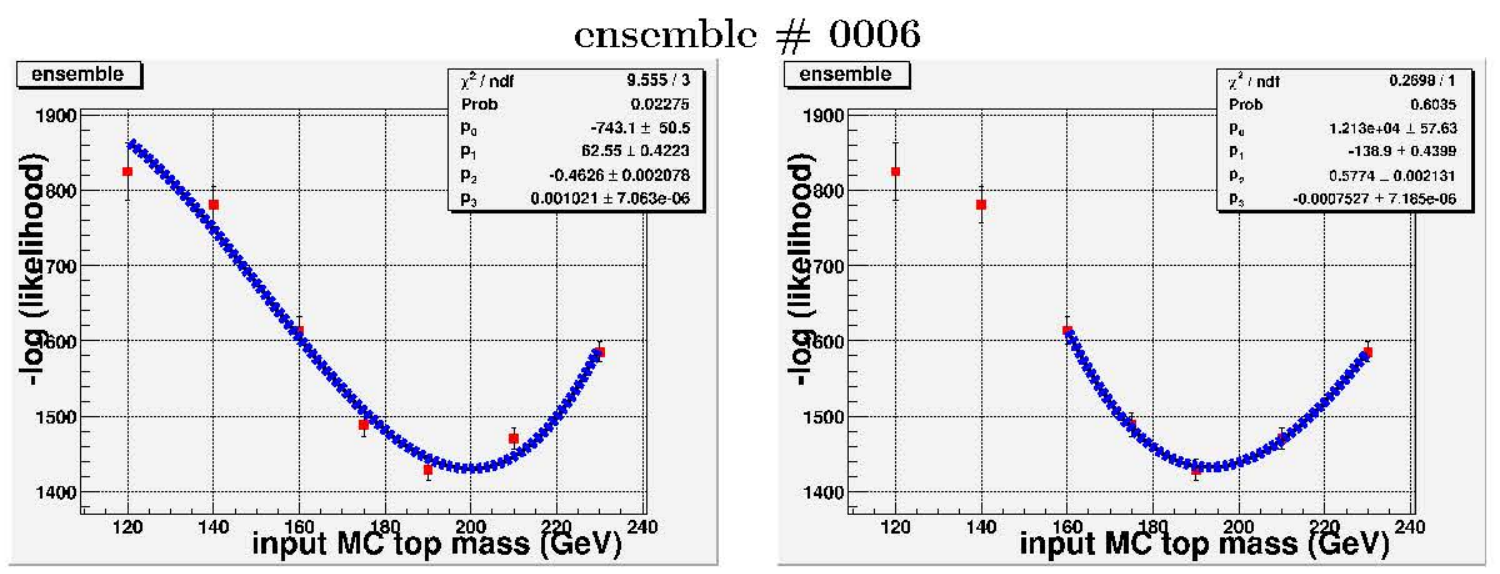

fitted $m_{t}=199.94_{+1.98}^{-2.02} \mathrm{GeV}$
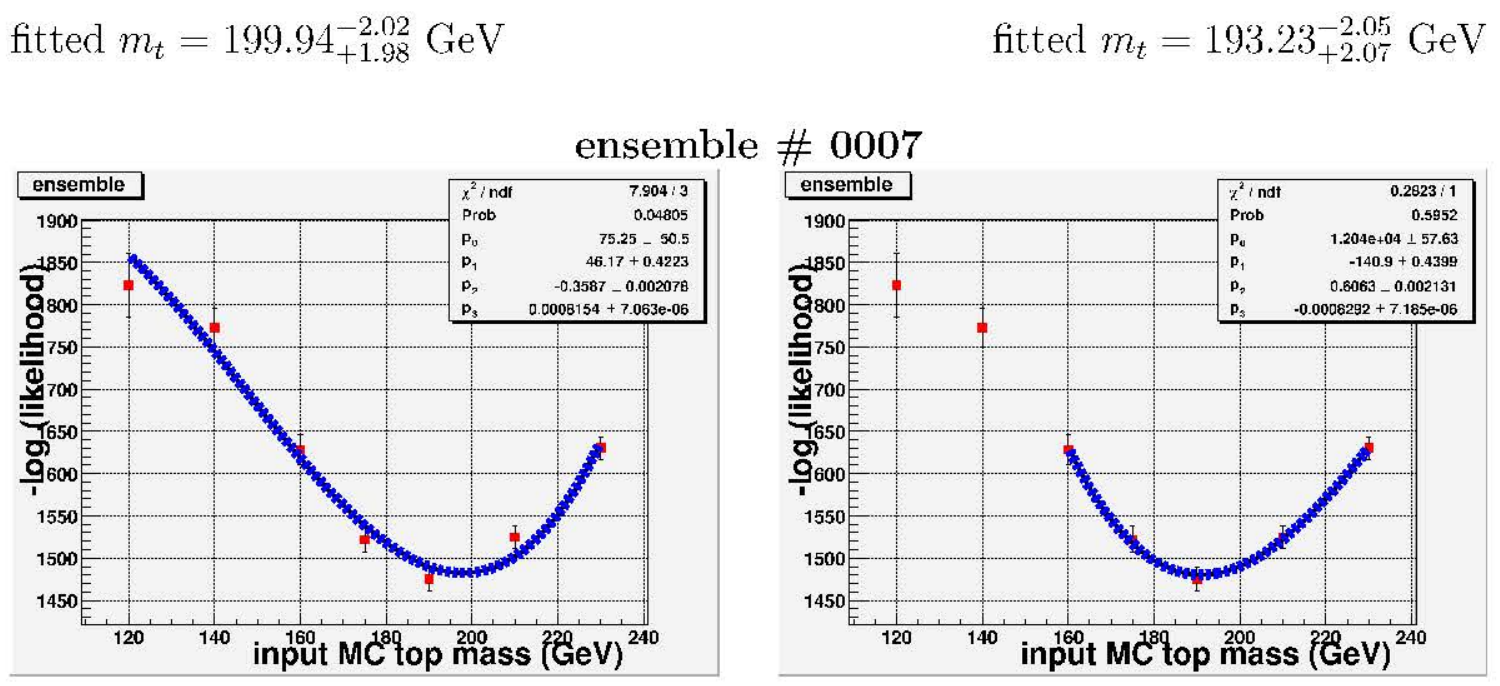

fitted $m_{t}=197.96_{+2.17}^{-2.20} \mathrm{GeV}$

fitted $m_{t}=190.95_{+2.15}^{-2.12} \mathrm{GeV}$
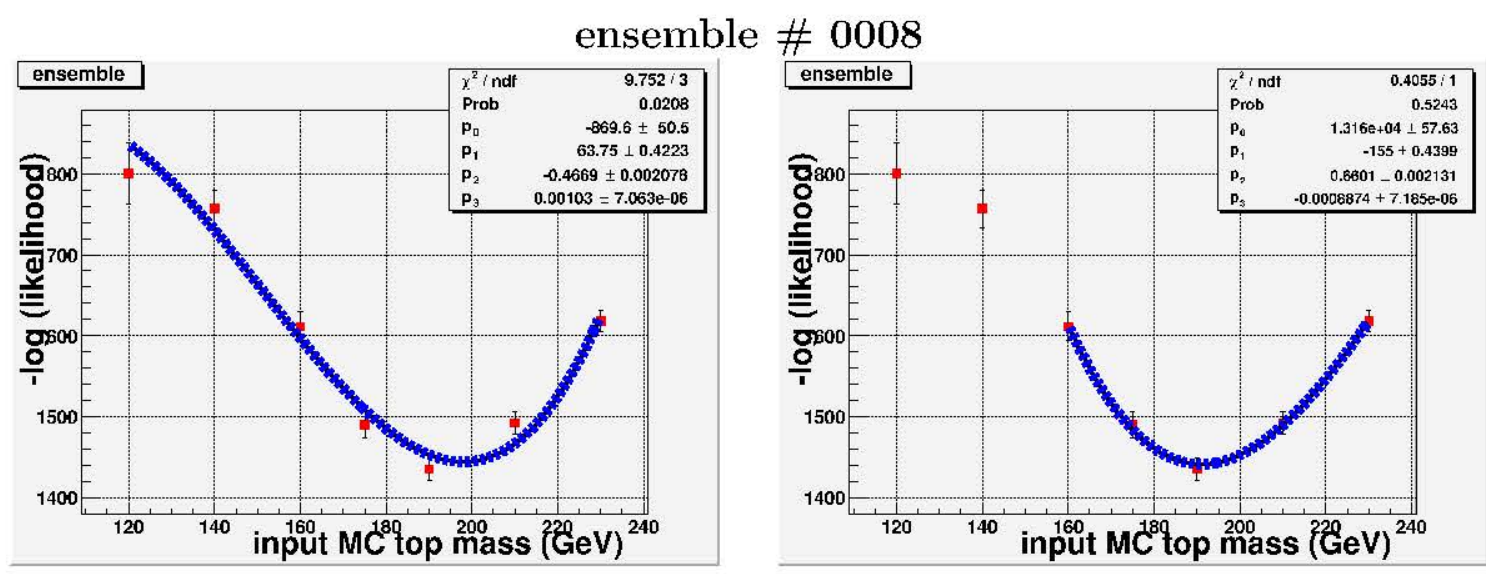

fit.ted $m_{t}=198.00_{+2.02}^{-2.05} \mathrm{GcV}$

fitted $m_{t}=191.04_{+2.00}^{-1.98} \mathrm{GeV}$ 


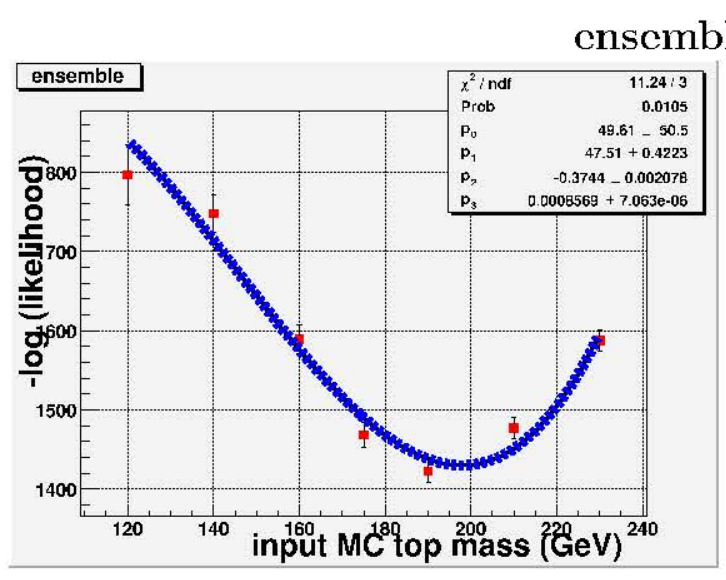

fitted $m_{t}=197.90_{+2.10}^{-2.13} \mathrm{GeV}$

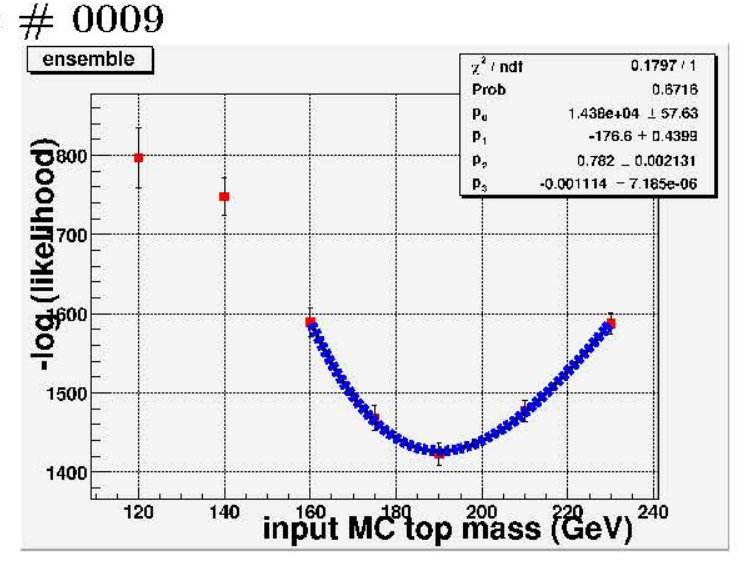

fitted $m_{t}=190.17_{+2.01}^{-2.01} \mathrm{GeV}$

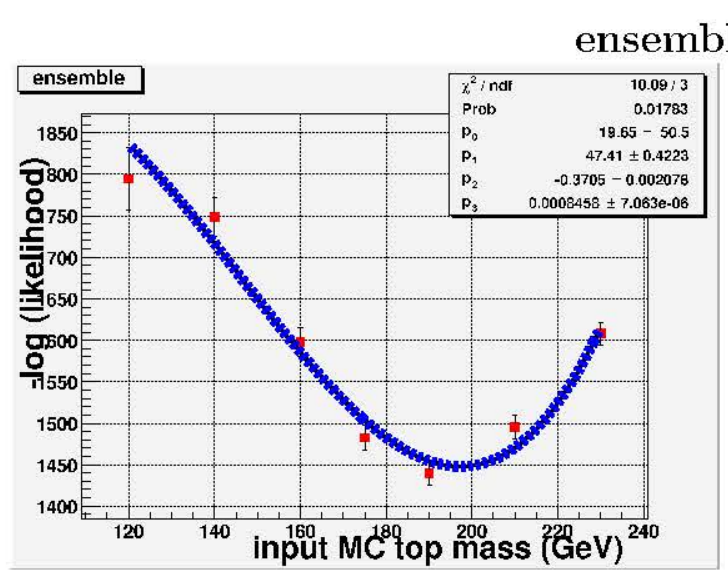

fitted $m_{t}=197.34_{+2.13}^{-2.16} \mathrm{GeV}$

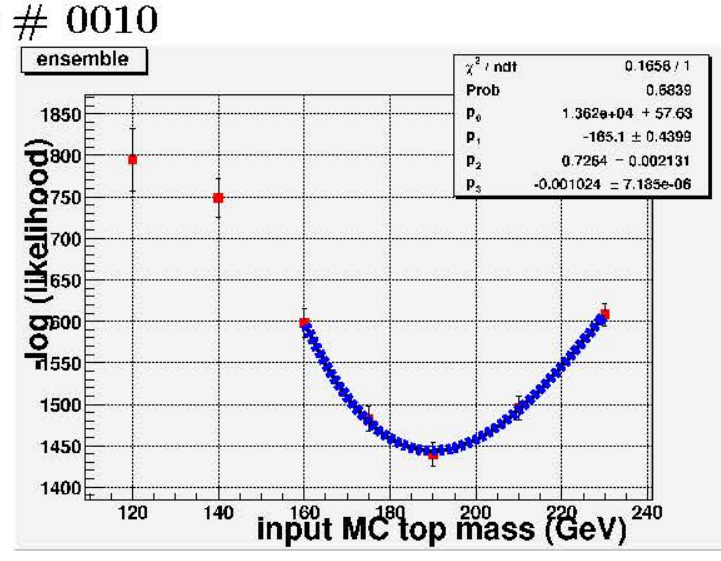

fitted $m_{t}=189.866_{+2.06}^{-2.03} \mathrm{GeV}$

Although these tests are using events having an input value of the top quark mass at $190 \mathrm{GcV}$, a similar discrepancy has been noticed for all other input valucs of the mass of the top quark. It is evident from the plots that for ensembles with large number statistics, a numerical fit within a narrow region yields a reasonable value of the MIF. When the ensemble has large number statistics, the response due to possible background events resembling the signal events of a specific input top quark mass is averaged out. However, for ensembles with small number statistics, this is not the casc. 


\section{Appendix I}

\section{Additional information from simulated ensemble studies:the MLE and pull distributions}

In this section, information about the MLE distributions and the corresponding pull distributions from unique cnscmbles are presented. Every cnsemble has signal and background processes multinomially distributed. The mean values of the purity (and contamination) is obtained from the cross-section measurement[52] in the respective channcls.

The pull of the distribution is defined as:

$$
\text { pull }=\left(\frac{\text { fitted } m_{t}-\mathrm{VC} m_{t}}{\sigma}\right)
$$

where $\sigma$ is the statistical uncertainty obtained from the log-likelihood fit. For this analysis the greater of the left statistical uncertainty and the right statistical uncertainty is used

$$
\sigma=\max \left(\sigma_{\text {stat. }}^{\text {left }}, \sigma_{\text {stat. }}^{\text {right }}\right)
$$

Figures I.1 through I.j represent the MLE and pull distributions from tests with events having the full detector simulation, in the e $\mu$ channel. Figures I.6 through 
I.10 correspond to similar tests in the ee channel. 

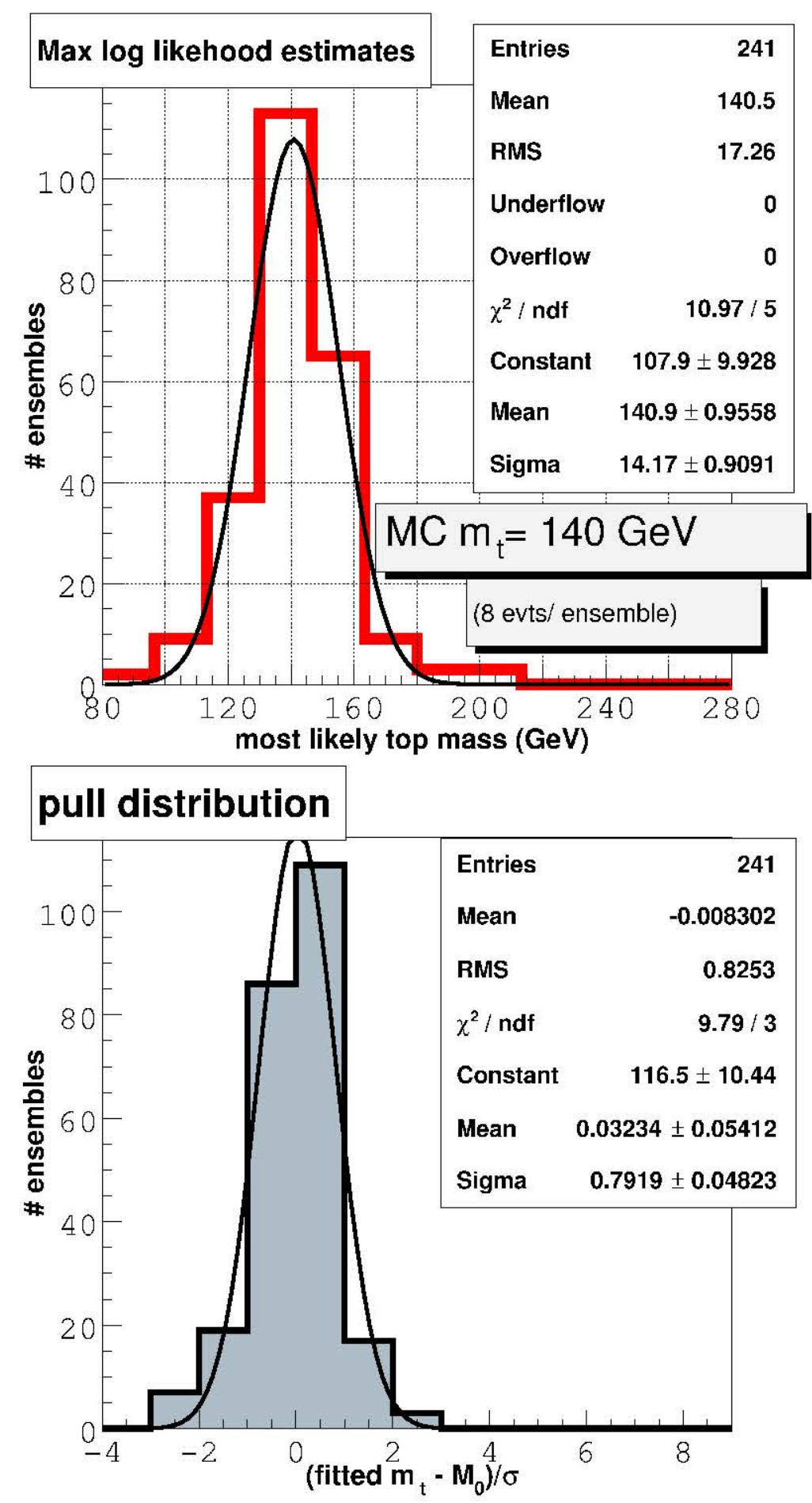

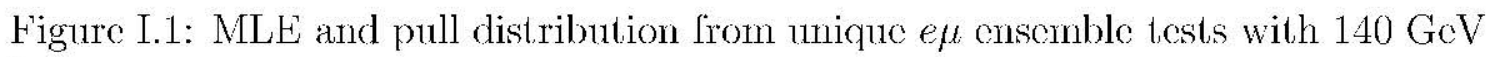
inpul $m_{t}$. 

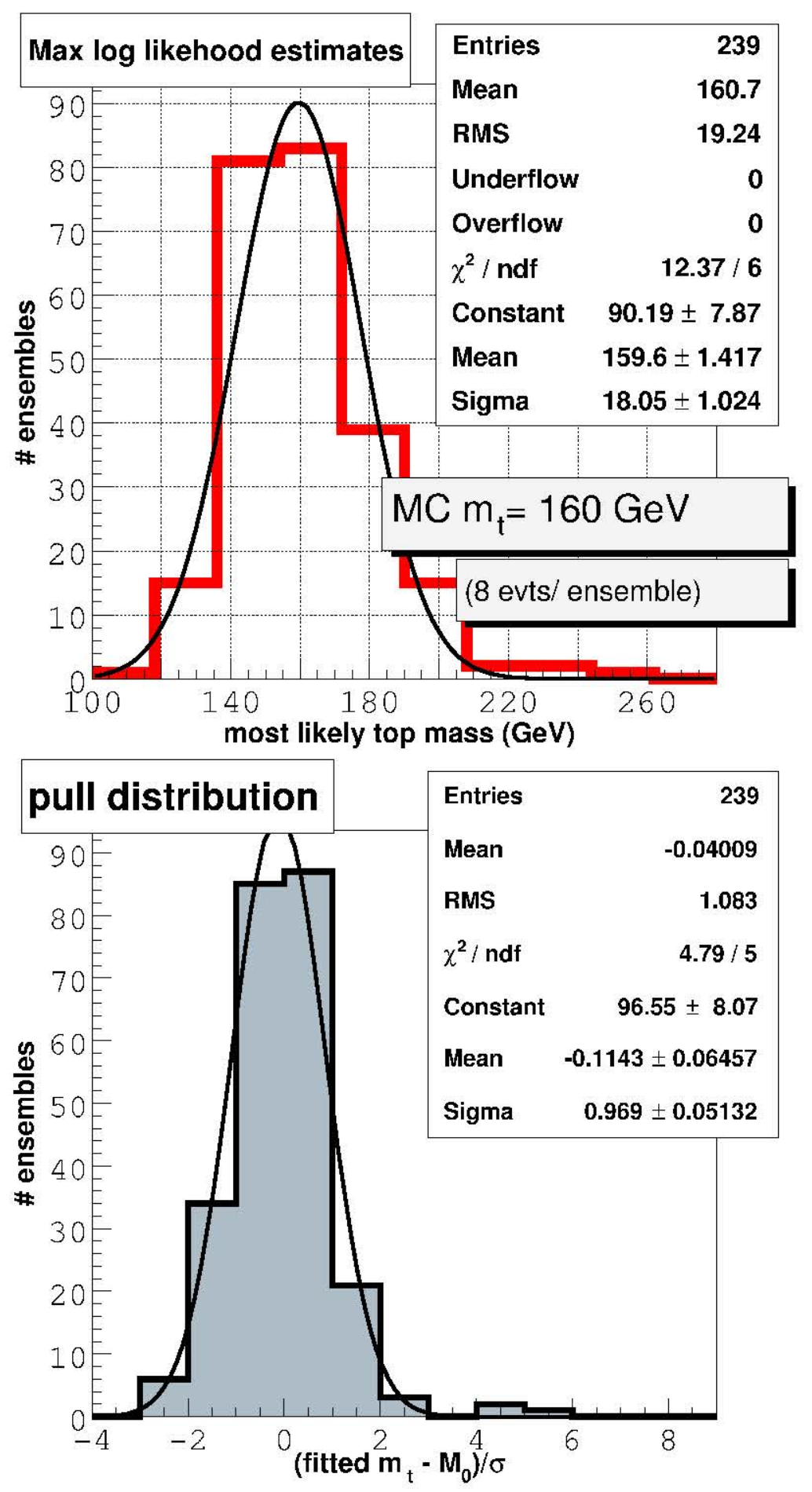

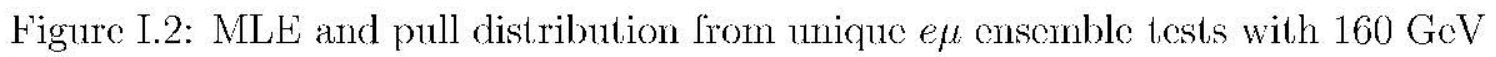
inpul $m_{t}$. 

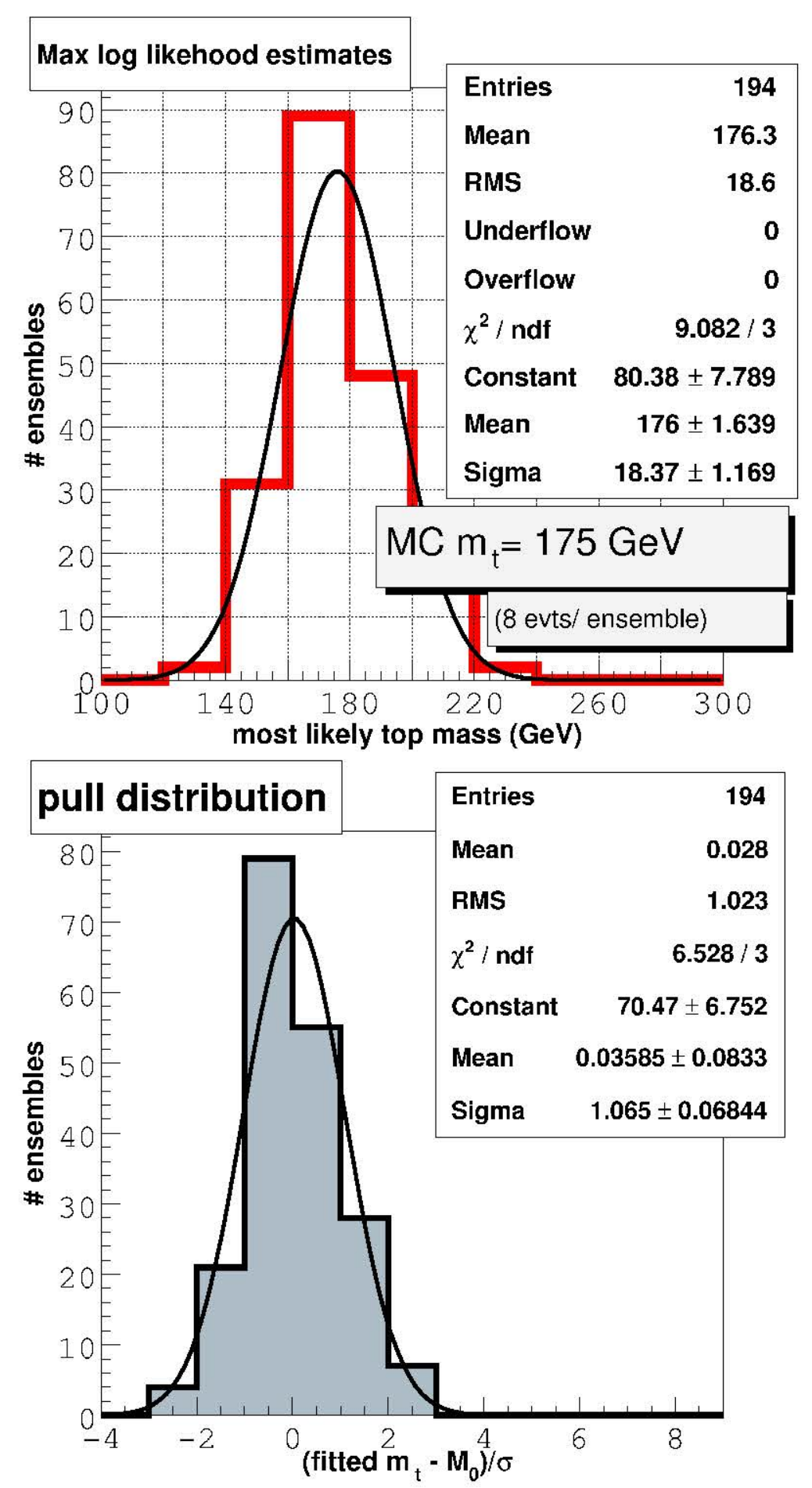

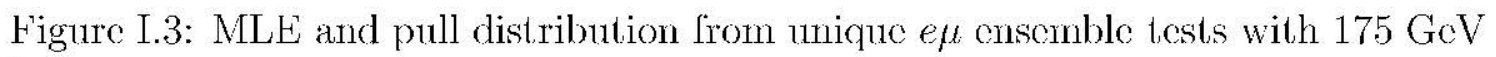
inpul $m_{t}$. 

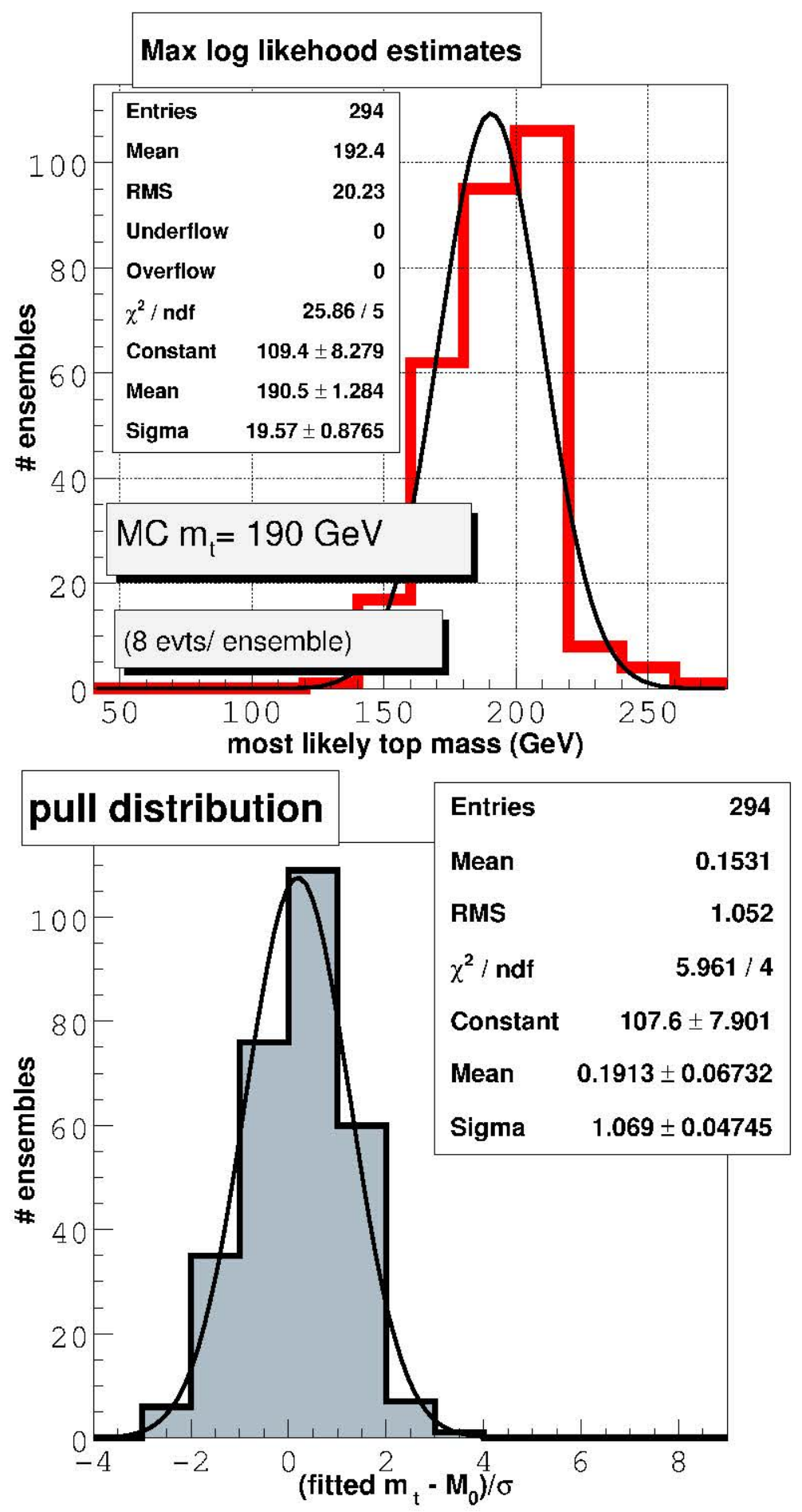

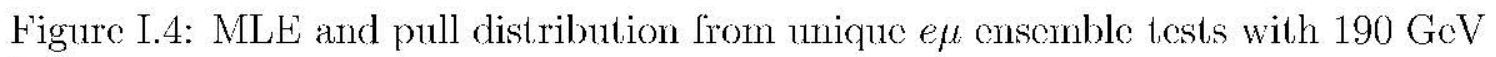
inpul $m_{t}$. 

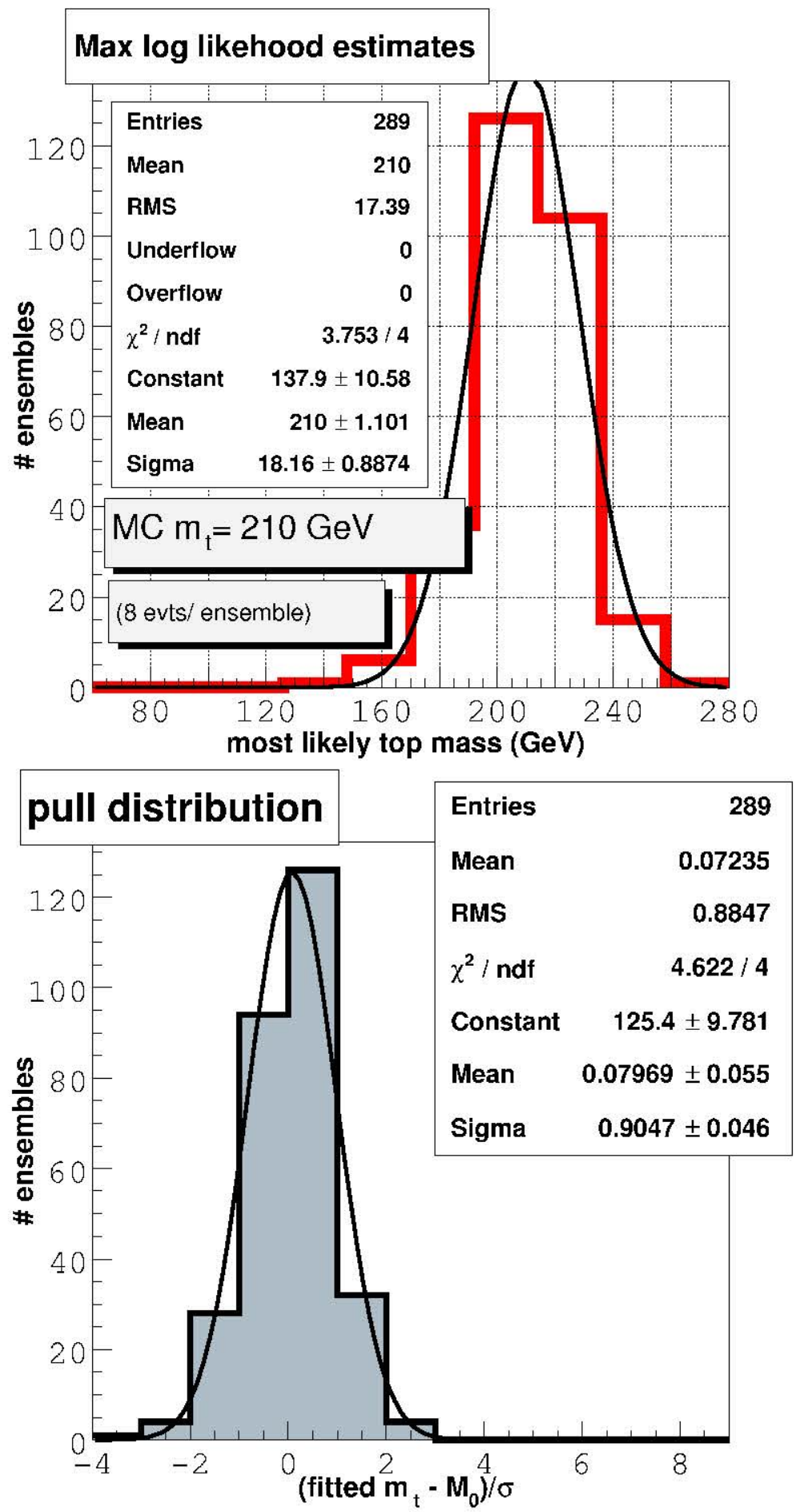

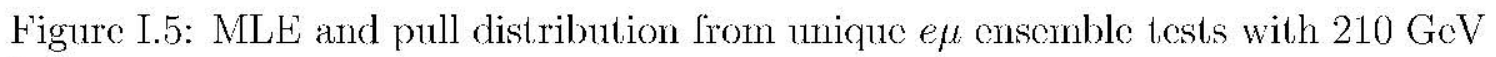
inpul $m_{t}$. 

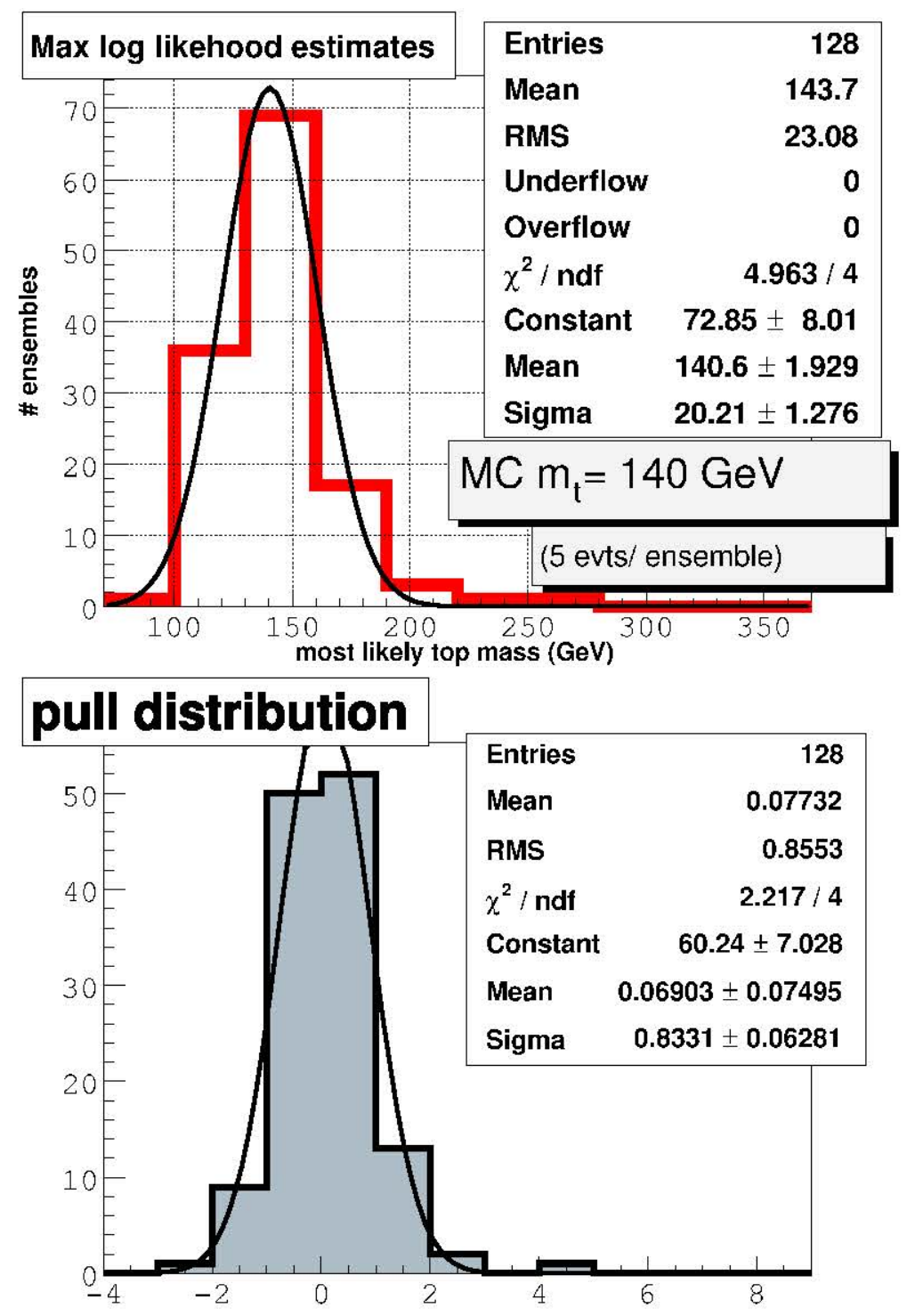

Figure I.6: MLE and pull distribution from unique ee ensemble tests with $140 \mathrm{GeV}$ input $m_{t}$. 

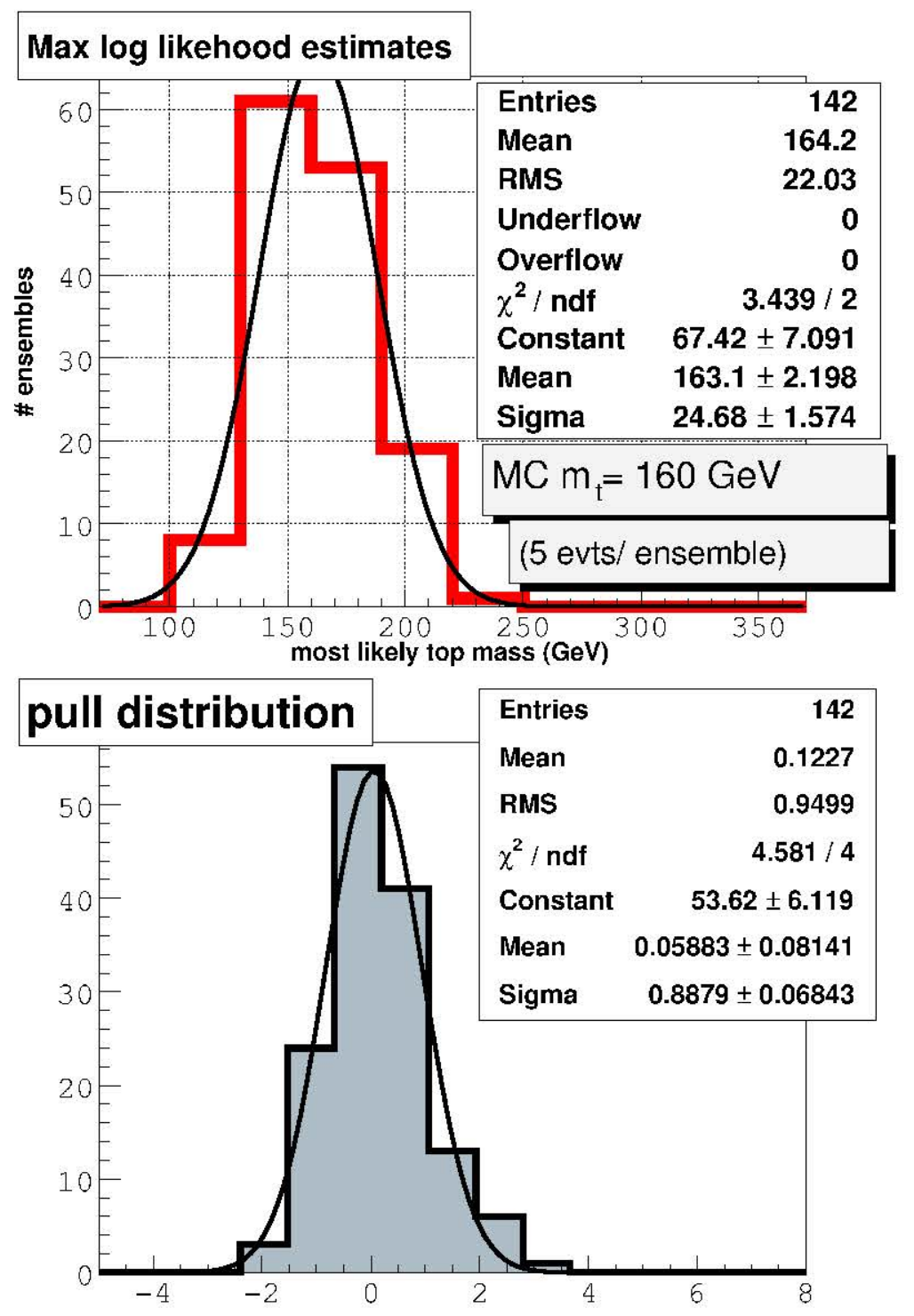

Figure I.7: MLE and pull distribution from unique ee ensemble tests with $160 \mathrm{GeV}$ input $m_{t}$. 

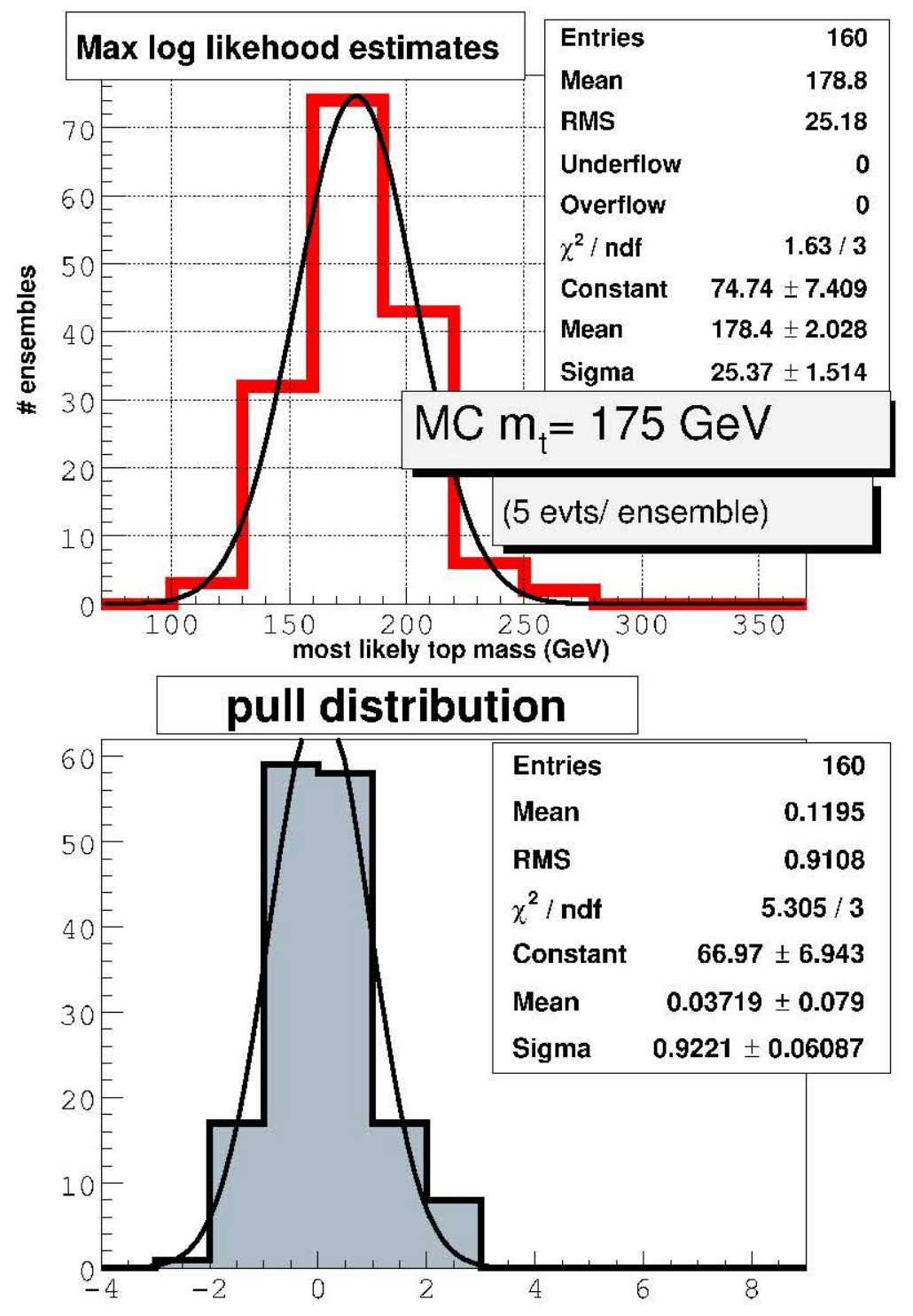

Figure I.8: MLE and pull distribution from unique ee ensemble tests with $175 \mathrm{GeV}$ input $m_{t}$. 

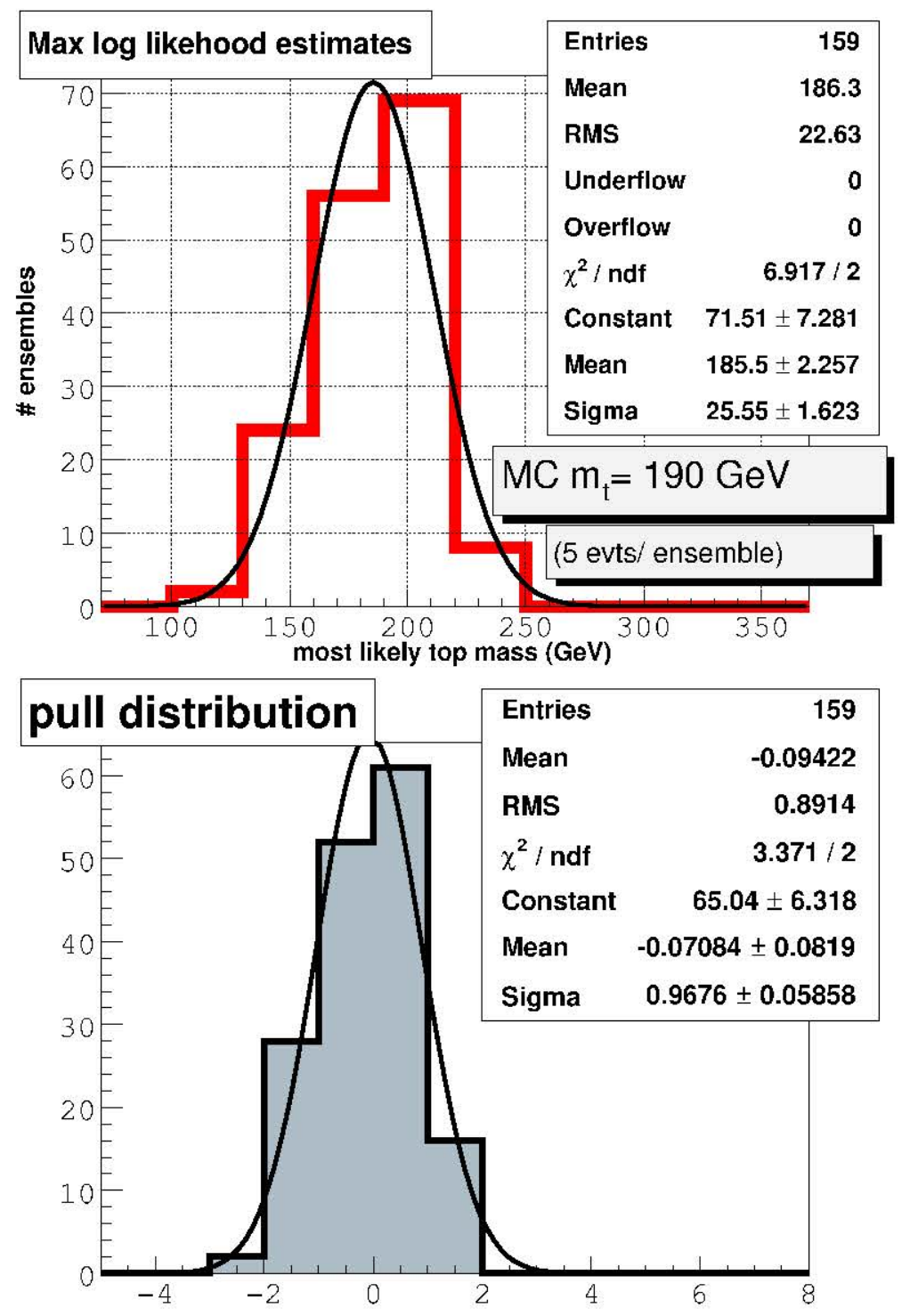

Figure I.9: MLE and pull distribution from unique ee ensemble tests with $190 \mathrm{GeV}$ input $m_{t}$. 

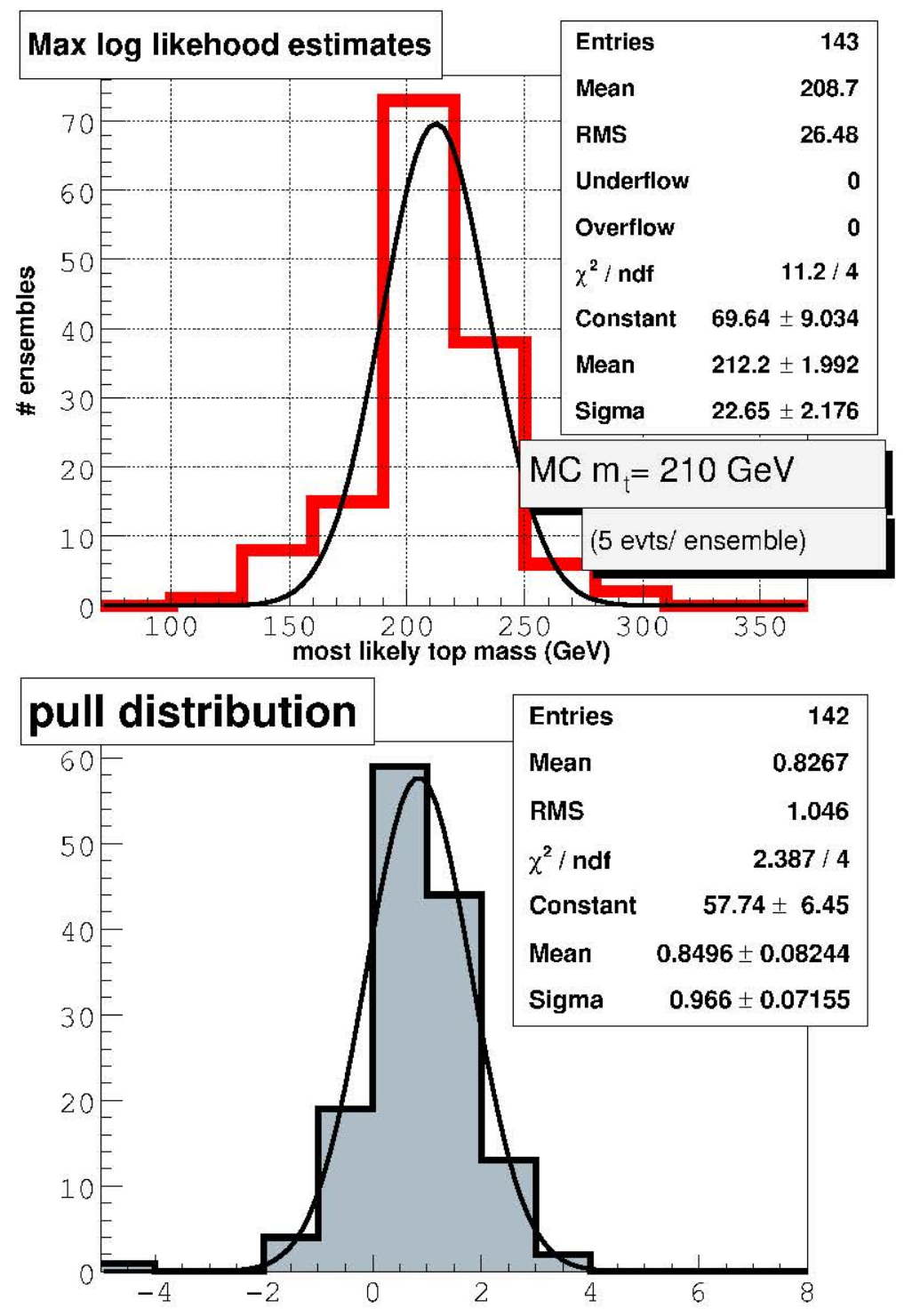

Figure I.10: MLE and pull distribution from unique ee ensemble tests with $210 \mathrm{GeV}$ input $m_{t}$. 


\section{Appendix $\mathbf{J}$}

\section{A study of the bias in ensemble calibration}

In this section, we study the effects when the composition of background processes is steadily increased in ensembles. The composition of various background processes in every cnscmble is multinomially varied and the mean background composition is kept constant. All results to follow are using di-electron ensembles with 5 events per cnscmble. Before maximal optimization of the di-clectron sclection cuts, the background contamination was $\sim 46 \%$. These tests contain $20 \%, 40 \%$, $60 \%$, and $80 \%$ of the nominal background contamination.

As the background contamination is increased in ensembles as well as templates, the calibration curve for the system deviates from that of the ideal curve having a unit slope and a null ollsct. Since the template method by definition must yicld an ideal calibration curve ${ }^{1}$, it is evident from these studies that using small number statistics in background templates is instrumental in producing larger point to point deviations. This results in a calibration curve which deviates from the nominal fitted curve of unit slope and a null offset.

1. Results using signal and $9.26 \%$ background cvents multinomially combined are

\footnotetext{
${ }^{1}$ This is verificd from the toy simulation studies, as well as signal only studies mentioned in Chapter 8 .
} 
shown in Table J.1 and Figure J.1.

\begin{tabular}{|l|l|l|l|l|l|}
\hline input MC $m_{t}$ & $\mathbf{1 4 0}$ & $\mathbf{1 6 0}$ & $\mathbf{1 7 5}$ & $\mathbf{1 9 0}$ & $\mathbf{2 1 0}$ \\
\hline$\left\langle m_{t}\right\rangle$ & 142.043 & 159.129 & 176.418 & 192.494 & 211.79 \\
\hline$\langle R M S$ of mean $>$ & 18.6365 & 17.7903 & 19.9562 & 21.5522 & 17.1098 \\
\hline$\left\langle\frac{R M S}{\sqrt{N_{\text {expt. }}}}\right\rangle$ & 1.89556 & 1.79888 & 2.01725 & 2.18134 & 1.73014 \\
\hline$\langle$ pull $>$ & -0.08 & -0.09 & -0.02 & +0.05 & +0.11 \\
\hline$\langle$ RMS of pull $>$ & 0.99 & 0.90 & 1.07 & 1.15 & 0.91 \\
\hline
\end{tabular}

Table J.1: Results from simulated cnsembles with $\sim 10 \%$ background contamination.

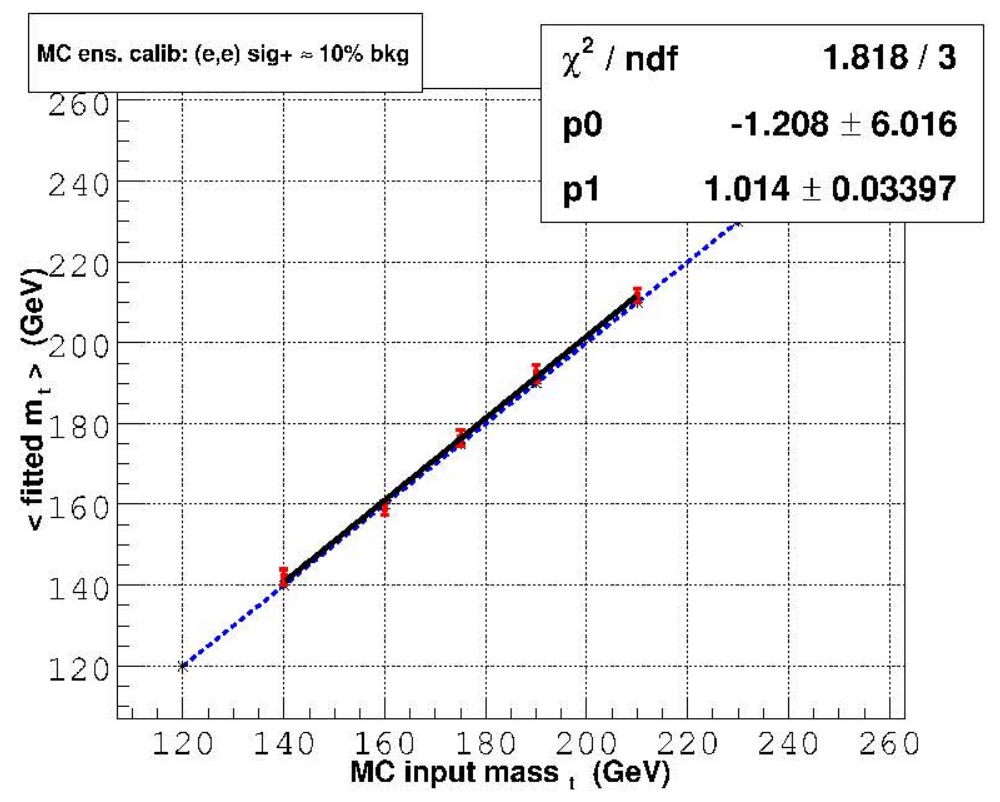

Figure J.1: Calibration curve for simulated cnscmbles with $\sim 10 \%$ background contamination.

2. Results using signal and $18.52 \%$ background cvents multinomially combined are shown in Table J.2 and Figure J.2.

3. Results using signal and $27.78 \%$ background events multinomially combined are shown in Table J.3 and Figure J.3. 


\begin{tabular}{|l|l|l|l|l|l|}
\hline input, MC $m_{t}$ & 140 & $\mathbf{1 6 0}$ & $\mathbf{1 7 5}$ & $\mathbf{1 9 0}$ & $\mathbf{2 1 0}$ \\
\hline$\left\langle m_{t}\right\rangle$ & 143.445 & 159.511 & 175.557 & 189.722 & 210.212 \\
\hline$\langle$ RMS of mean $\rangle$ & 18.6004 & 19.886 & 20.6076 & 23.6402 & 20.8660 \\
\hline $\begin{array}{l}\text { RMS } \\
\left.\sqrt{N_{\text {cxpt. }}}\right\rangle\end{array}$ & 1.93229 & 2.03012 & 2.09062 & 2.11023 & 2.12545 \\
\hline$\langle$ pull $>$ & -0.123 & -0.176. & -0.039 & +0.041 & +0.108 \\
\hline$\langle$ RMS of pull $\rangle$ & 0.97124 & 0.954 & 1.07498 & 1.1863 & 1.017 \\
\hline
\end{tabular}

Table J.2: Results from simulated ensembles with $\sim 19 \%$ background contamination.

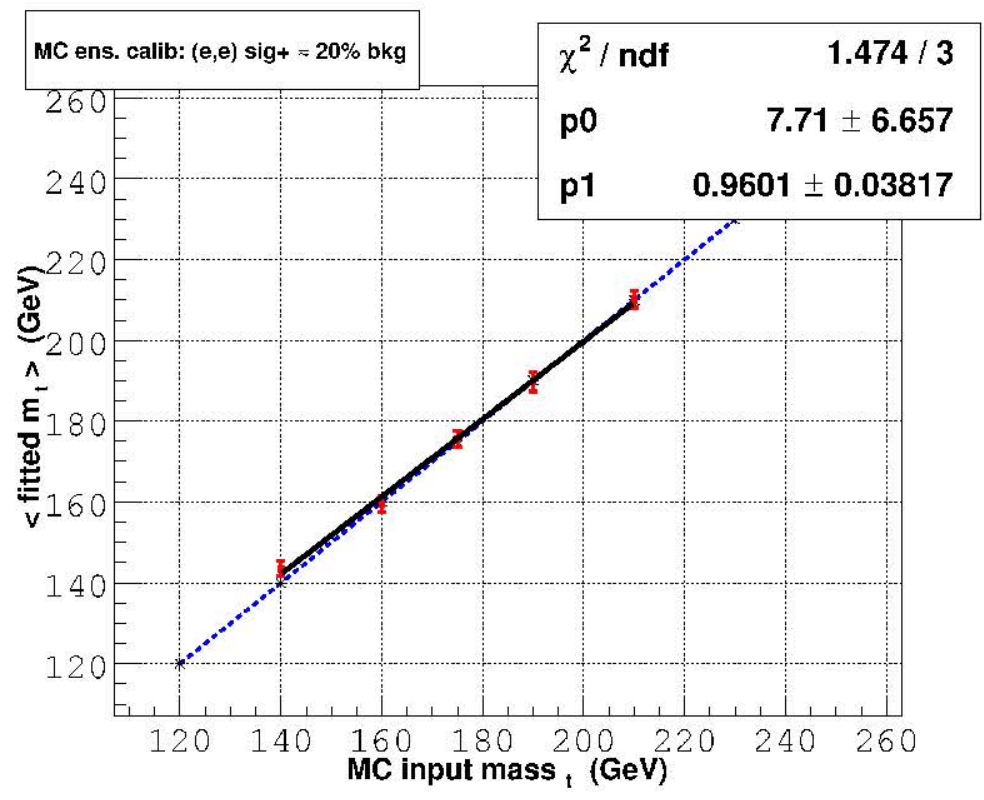

Figure J.2: Calibration curve for simulated ensembles with $\sim 19 \%$ background contamination.

4. Results using signal and $37.04 \%$ background events multinomially combined are shown in Table J.4 and Figure J.4. 


\begin{tabular}{|l|l|l|l|l|l|}
\hline input MC $m_{t}$ & $\mathbf{1 4 0}$ & $\mathbf{1 6 0}$ & $\mathbf{1 7 5}$ & $\mathbf{1 9 0}$ & $\mathbf{2 1 0}$ \\
\hline$\left.<m_{l}\right\rangle$ & 116.89 & 159.83 & 174.797 & 189.81 & 209.323 \\
\hline$<R M S$ of mean $>$ & 21.1991 & 20.648 & 22.7066 & 25.3922 & 24.0048 \\
\hline$<\frac{R M S}{\left.\sqrt{N_{\text {expt. }}}\right\rangle}$ & 2.21847 & 2.13546 & 2.32161 & 2.61497 & 2.4843 \\
\hline$<$ pull $>$ & -0.2057 & -0.1328 & -0.0497 & +0.0274 & +0.0981 \\
\hline$<$ RMS of pull $>$ & 1.08555 & 0.9890 & 1.11345 & 1.2123 & 1.0968 \\
\hline
\end{tabular}

Table J.3: Results from simulated ensembles with $\sim 28 \%$ background contamination.

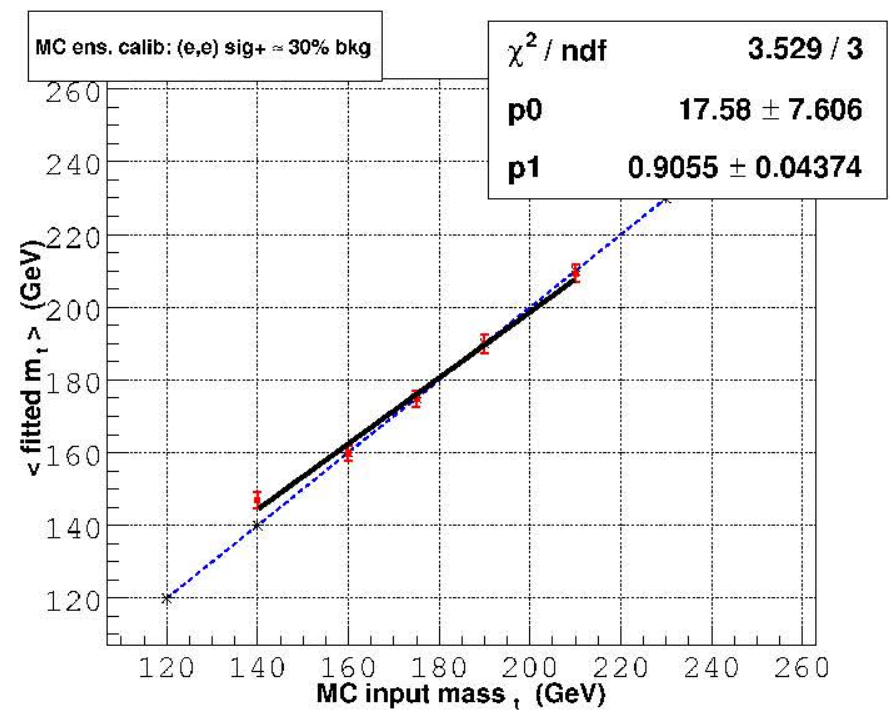

Figure J.3: Calibration curve for simulated ensembles with $\sim 28 \%$ background contamination.

\begin{tabular}{|l|l|l|l|l|l|}
\hline inpul MC $m_{l}$ & $\mathbf{1 4 0}$ & $\mathbf{1 6 0}$ & $\mathbf{1 7 5}$ & $\mathbf{1 9 0}$ & $\mathbf{2 1 0}$ \\
\hline$\left.<m_{t}\right\rangle$ & 148.784 & 160.949 & 175.083 & 188.456 & 205.926 \\
\hline$<$ RMS of mean $>$ & 23.7513 & 22.9397 & 23.0111 & 26.5395 & 26.5679 \\
\hline$<\frac{\text { RMS }}{\sqrt{N_{\text {expt. }}}>}$ & 2.53969 & 2.38601 & 2.3644 & 2.75919 & 2.77282 \\
\hline$<$ pull $>$ & -0.168 & -0.1298 & -0.049 & +0.0117 & +0.1089 \\
\hline$<$ RMS of pull $>$ & 1.08151 & 1.02953 & 1.08215 & 1.2161 & 1.15357 \\
\hline
\end{tabular}

Table J.4: Results from simulated ensembles with $\sim 37 \%$ background contamination. 


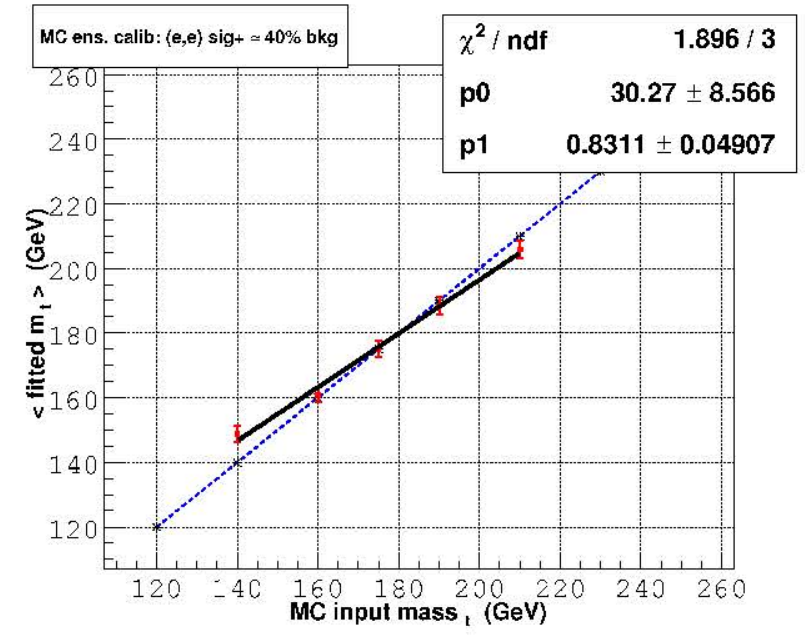

Figure J.4: Calibration curve for simulated ensembles with $\sim 37 \%$ background contamination. 
In the next two sets of tests, the number of background events have been kept fixed for each ensemble, and the effects of bias in the calibration are studied. It is observed that the nature of bias when the background composition is fluctuated is different from when the background composition is kept fixed. In the former, the slope of the calibration curve deviates about ${ }^{2} \sim 175 \mathrm{GeV}$, whereas it is different in the latter.

5. Results from ensemble tests with signal and a fixed background combination of 20\% are shown in Table J.5 and Figure J.5, while the results from cnscmbles with a fixed background contamination of $40 \%$ are shown in Table J.6 and Figure J.6.

\begin{tabular}{|l|l|l|l|l|l|}
\hline input MC $m_{t}$ & $\mathbf{1 4 0}$ & $\mathbf{1 6 0}$ & $\mathbf{1 7 5}$ & $\mathbf{1 9 0}$ & $\mathbf{2 1 0}$ \\
\hline$\left\langle m_{t}\right\rangle$ & 142.418 & 158.359 & 175.962 & 189.59 & 209.281 \\
\hline$\langle$ RMS of mean $>$ & 20.7757 & 18.2517 & 21.3377 & 23.7459 & 20.666 \\
\hline$\left\langle\frac{\text { RMS }}{\sqrt{N_{\text {expt. }}}}\right\rangle$ & 2.13514 & 1.86779 & 2.16331 & 2.42229 & 2.10341 \\
\hline$\langle$ pull $>$ & -0.0663 & -0.0966 & -0.0603 & +0.0278 & 0.0923 \\
\hline$\langle$ RMS of pull $\rangle$ & 0.9878 & 0.889904 & 1.10687 & 1.18627 & 1.028 \\
\hline
\end{tabular}

Table J.5: Results from simulated ensembles with (fixed) $20 \%$ background contarnination.

\begin{tabular}{|l|l|l|l|l|l|}
\hline input MC $m_{t}$ & $\mathbf{1 4 0}$ & $\mathbf{1 6 0}$ & $\mathbf{1 7 5}$ & $\mathbf{1 9 0}$ & $\mathbf{2 1 0}$ \\
\hline$\left\langle m_{t}\right\rangle$ & 149.364 & 162.855 & 179.763 & 193.972 & 209.97 \\
\hline$\langle$ RMS of mean $>$ & 30.4177 & 27.5155 & 25.1868 & 27.9157 & 23.172 \\
\hline $\begin{array}{l}\text { RMS } \\
\left.\sqrt{N_{\text {c.xpt. }}}\right\rangle\end{array}$ & 3.32732 & 2.93149 & 2.63378 & 2.91491 & 2.15823 \\
\hline$\langle$ pull $>$ & -0.1265 & -0.068725 & +0.033253 & +0.0168 & +0.0974 \\
\hline$<$ RMS of pull $\rangle$ & 1.05924 & 1.02692 & 1.00943 & 1.12753 & 0.932 \\
\hline
\end{tabular}

Table J.6: Results from simulated ensembles with (fixcd) 10\% background contamination.

\footnotetext{
${ }^{2}$ Within statistical fluctuations.
} 


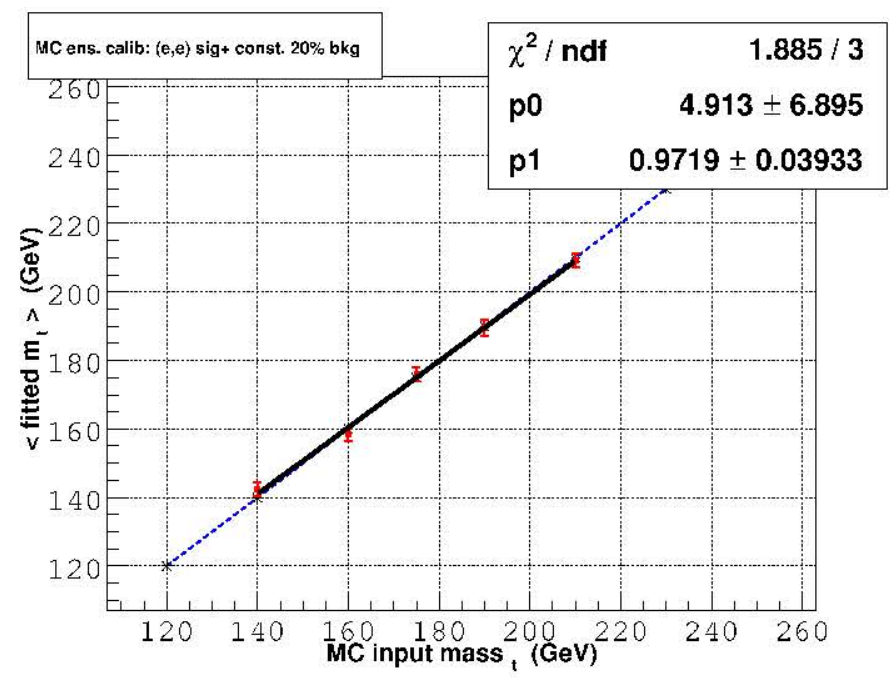

Figure J.5: Calibration curve for simulated ensembles with fixed $20 \%$ background contamination.

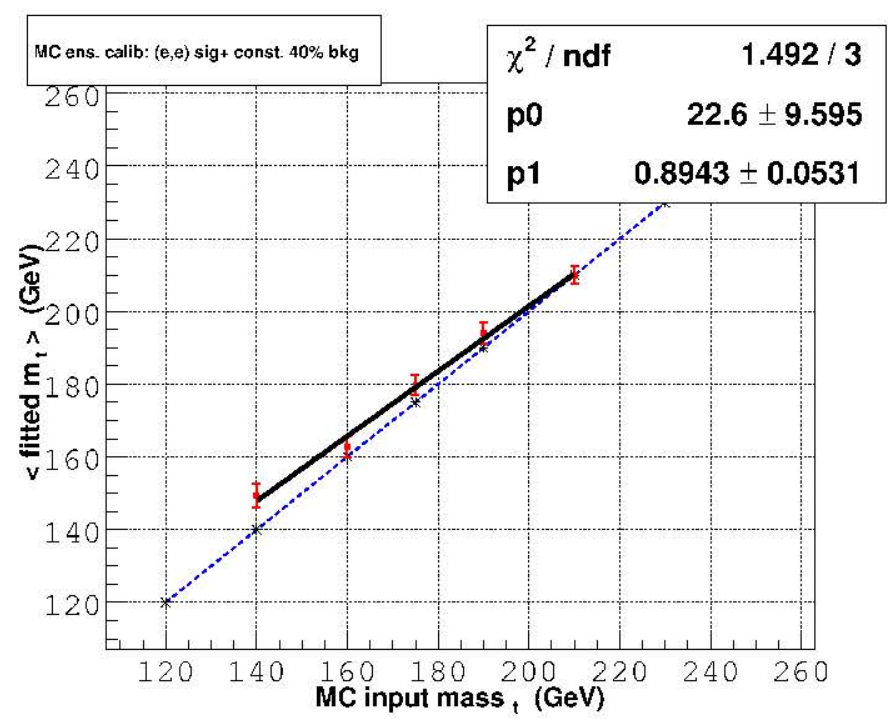

Figure J.6: Calibration curve for simulated ensembles with fixed $10 \%$ background contamination. 


\section{Appendix $\mathrm{K}$}

\section{Kinematic information of candidate events}

This section contains the 4-vectors of the objects from the candidate events. The 4-vectors of the jets are obtained after the $\eta$ dependent scale corrections, and the parton level corrections have becn applicd. The $m_{p e a k}$ values obtained from the event kinematics are depicted in the captions. The listed 4 -vectors have been smeared using the relevant resolution parameters listed in Chapter 7, Tables 7.11, 7.12 and 7.13 to extract the $m_{p e a k}$ value for each event. First the kinematics of the eight $e \mu$ events are presented in the Tables K.1 through K.8. The kinematics of the five candidate events selected in the ee channel are now listed in Tables K.9 through K.13.

\begin{tabular}{l|r|r|r|r}
\hline objcct & $p_{x}$ & $p_{y}$ & $p_{z}$ & $|\vec{p}|$ \\
\hline electron & -10.52 & 11.97 & 20.42 & 25.90 \\
\hline muon & 8.43 & 55.96 & -21.29 & 60.47 \\
\hline jct 1 & 11.52 & -76.28 & 30.07 & 83.31 \\
\hline jct 2 & -48.31 & 21.48 & -49.82 & 73.1476 \\
\hline$\phi_{T}$ & 37.64 & -7.68 & $N A$ & $N A$ \\
\hline
\end{tabular}

Table K.1: Four vectors of objects: cvent 1997007 in run 168393 . The $m_{\text {peak }}$ value obtained from the event is $145 \mathrm{GeV}$. 


\begin{tabular}{l|r|r|r|r}
\hline objcct & $p_{x}$ & $p_{y}$ & $p_{z}$ & $|\vec{p}|$ \\
\hline electron & -43.84 & 129.29 & -5.66 & 136.64 \\
\hline muon & -2.62 & 29.47 & 15.18 & 33.25 \\
\hline jet 1 & 39.17 & -79.31 & 77.93 & 118.44 \\
\hline jot 2 & -3.15 & -87.81 & 189.97 & 209.71 \\
\hline jet 3 & -39.42 & -25.74 & 36.96 & 60.38 \\
\hline$\not_{T}$ & 84.24 & 4.42 & $N A$ & $N A$ \\
\hline
\end{tabular}

Table K.2: Four vectors ol objects: cvent 8710859 in run 171901 . The $m_{\text {peak value }}$ obtained from the event is $269 \mathrm{GeV}$.

\begin{tabular}{l|r|r|r|r}
\hline object & $p_{x}$ & $p_{y}$ & $p_{z}$ & $|\vec{p}|$ \\
\hline electron & -25.26 & 44.67 & -67.80 & 85.04 \\
\hline muon & 61.66 & -51.29 & -41.16 & 90.15 \\
\hline jet 1 & 52.24 & -148.75 & -15.55 & 159.01 \\
\hline jet 2 & -82.47 & 79.21 & -12.11 & 122.31 \\
\hline$\not_{T}$ & -3.46 & 77.84 & $N \Lambda$ & $N \Lambda$ \\
\hline
\end{tabular}

Table K.3: Four vectors of objects: event 15259654 in run 177826 . The $m_{\text {peak }}$ value obtained from the event is $140 \mathrm{GeV}$. 


\begin{tabular}{l|r|r|r|r}
\hline object & $p_{x}$ & $p_{y}$ & $p_{z}$ & $|\vec{p}|$ \\
\hline electron & -66.75 & -86.52 & -75.01 & 132.54 \\
\hline muon & 70.46 & 101.28 & 21.55 & 125.25 \\
\hline jct 1 & 61.12 & 23.46 & 24.55 & 70.71 \\
\hline jet 2 & -45.96 & -2.05 & 15.99 & 49.63 \\
\hline$\not_{T}$ & -15.53 & -37.61 & $N A$ & $N A$ \\
\hline
\end{tabular}

Table K.4: Four vectors of objects: event 37315438 in run 178159 . The $m_{\text {peak }}$ value obtained from the event is $133 \mathrm{GeV}$.

\begin{tabular}{l|r|r|r|r}
\hline object & $p_{x}$ & $p_{y}$ & $p_{z}$ & $|\vec{p}|$ \\
\hline clcctron & 15.56 & -2.80 & 5.53 & 16.75 \\
\hline mulon & -23.57 & -46.33 & 79.71 & 95.16 \\
\hline jet 1 & 33.11 & -105.86 & -5.39 & 111.92 \\
\hline jet 2 & 44.84 & 21.32 & -9.49 & 50.98 \\
\hline$p_{T}$ & -62.47 & 139.01 & $N A$ & $N A$ \\
\hline
\end{tabular}

Table K.5: Four vectors of objects: event 8735139 in run 178733 . The $m_{\text {pcak }}$ value obtained from the event is $162 \mathrm{GeV}$.

\begin{tabular}{l|r|r|r|r}
\hline object & $p_{x}$ & $p_{y}$ & $p_{z}$ & $|\vec{p}|$ \\
\hline clectron & 18.64 & -24.12 & -37.44 & 48.29 \\
\hline muon & 4.98 & -52.30 & -102.48 & 115.17 \\
\hline jet 1 & 32.39 & 47.30 & 20.91 & 61.56 \\
\hline jet 2 & -24.54 & 33.41 & -6.60 & 42.57 \\
\hline$\not_{T}$ & -27.50 & -11.84 & $N A$ & $N A$ \\
\hline
\end{tabular}

Table K.6: Four vectors of objects: event 11709332 in run 179141 . The $m_{\text {peak }}$ value obtained from the event is $161 \mathrm{GeV}$.

\begin{tabular}{l|r|r|r|r}
\hline object & $p_{x}$ & $p_{y}$ & $p_{z}$ & $|\vec{p}|$ \\
\hline clcctron & 72.87 & -6.49 & -51.73 & 89.60 \\
\hline muon & -70.11 & -31.30 & -19.55 & 79.23 \\
\hline jct 1 & 98.72 & 11.25 & -16.55 & 109.91 \\
\hline jet 2 & -85.37 & 63.62 & -47.45 & 117.10 \\
\hline$\phi_{T}$ & -23.18 & -65.32 & $N \Lambda$ & $N \Lambda$ \\
\hline
\end{tabular}

Table K.7: Four vectors of objects: cvent 26386170 in run 179195 . The $m_{\text {peak }}$ valuc obtained from the event is $164 \mathrm{GeV}$. 


\begin{tabular}{l|r|r|r|r}
\hline object & $p_{x}$ & $p_{y}$ & $p_{z}$ & $|\vec{p}|$ \\
\hline electron & -36.72 & -13.31 & -6.85 & 39.66 \\
\hline muon & -38.99 & -5.06 & 32.17 & 50.80 \\
\hline jct 1 & 122.95 & 19.39 & -13.18 & 126.12 \\
\hline jet 2 & -78.44 & 9.01 & 147.68 & 167.89 \\
\hline$\not p_{T}$ & 27.25 & -10.27 & $N A$ & $N A$ \\
\hline
\end{tabular}

Table K.8: Four vectors of objects: event 19617819 in run 179331. The $m_{\text {peak }}$ value obtained from the event is $214 \mathrm{GeV}$.

\begin{tabular}{l|r|r|r|r}
\hline object & $p_{x}$ & $p_{y}$ & $p_{z}$ & $|\vec{p}|$ \\
\hline clectron & -19.16 & 51.92 & -2.41 & 55.50 \\
\hline cloctron & -18.68 & -6.97 & 9.30 & 22.01 \\
\hline jet 1 & -102.73 & 11.50 & -39.61 & 112.23 \\
\hline jet 2 & 39.61 & -12.49 & 56.37 & 70.41 \\
\hline$\not_{T}$ & 100.89 & -45.16 & $N A$ & $N A$ \\
\hline
\end{tabular}

Table K.9: Four vectors of objects: event 121971122 in run 166779. The $m_{\text {peak }}$ value obtained from the event is $150 \mathrm{GeV}$.

\begin{tabular}{l|r|r|r|r}
\hline object & $p_{x}$ & $p_{y}$ & $p_{z}$ & $|\vec{p}|$ \\
\hline clectron & -10.3578 & 66.63 & 7.15 & 67.81 \\
\hline electron & 42.27 & -40.69 & 71.60 & 92.57 \\
\hline jet 1 & -75.16 & 34.39 & 43.68 & 94.11 \\
\hline jct 2 & -11.25 & -32.4303 & -17.89 & 39.42 \\
\hline$\not p_{T}$ & 10.61 & -16.69 & $N A$ & $N A$ \\
\hline
\end{tabular}

Table K.10: Four vectors of objects: event 13869716 in run 177681 . The $m_{\text {peak }}$ value obtained from the event is $114 \mathrm{GcV}$.

\begin{tabular}{l|r|r|r|r}
\hline object & $p_{x}$ & $p_{y}$ & $p_{z}$ & $|\vec{p}|$ \\
\hline clectron & 19.89 & -58.52 & -11.78 & 62.92 \\
\hline electron & -15.47 & -9.17 & -4.38 & 18.51 \\
\hline jct 1 & 73.21 & 32.65 & 88.88 & 120.07 \\
\hline jet 2 & -3.62 & 21.93 & -96.35 & 99.12 \\
\hline$\not_{T}$ & -78.67 & 12.66 & $N \Lambda$ & $N \Lambda$ \\
\hline
\end{tabular}

Table K.11: Four vectors of objects: cvent 26229011 in run 178152. The $m_{p e a k}$ valuc obtained from the event is $183 \mathrm{GeV}$. 


\begin{tabular}{l|r|r|r|r}
\hline object & $p_{x}$ & $p_{y}$ & $p_{z}$ & $|\vec{p}|$ \\
\hline electron & 14.98 & 96.48 & 28.50 & 101.71 \\
\hline electron & 13.79 & 12.94 & -3.23 & 19.18 \\
\hline jet 1 & 99.59 & -81.19 & 173.16 & 216.01 \\
\hline jct 2 & -40.88 & -32.54 & 35.73 & 63.82 \\
\hline$\not_{T}$ & -98.47 & 7.38 & $N A$ & $N A$ \\
\hline
\end{tabular}

Table K.12: Four vectors of objects: cvent 13511001 in run 178177 . The $m_{\text {peak }}$ value obtained from the event is $192 \mathrm{GeV}$.

\begin{tabular}{l|r|r|r|r}
\hline object & $p_{x}$ & $p_{y}$ & $p_{z}$ & $|\vec{p}|$ \\
\hline electron & -63.76 & 82.76 & -150.36 & 183.10 \\
\hline electron & -11.92 & -40.98 & -44.27 & 61.50 \\
\hline jct 1 & 65.81 & -52.54 & -149.72 & 172.58 \\
\hline jet 2 & -12.91 & 67.16 & -18.73 & 72.14 \\
\hline jet 3 & 15.58 & 24.32 & -58.98 & 66.02 \\
\hline$\not \phi_{T}$ & -27.17 & -69.98 & $N A$ & $N A$ \\
\hline
\end{tabular}

Table K.13: Four vectors of objects: event 14448436 in run 180326 . The $m_{\text {peak }}$ value obtained from the event is $162 \mathrm{GeV}$. 


\section{Appendix L}

\section{Normalized weight distribution of candidate events}

The weight distributions of the five di-electron candidate events are illustrated in the Figures L.1 and L.2.

Run, Event 178152, 26229014

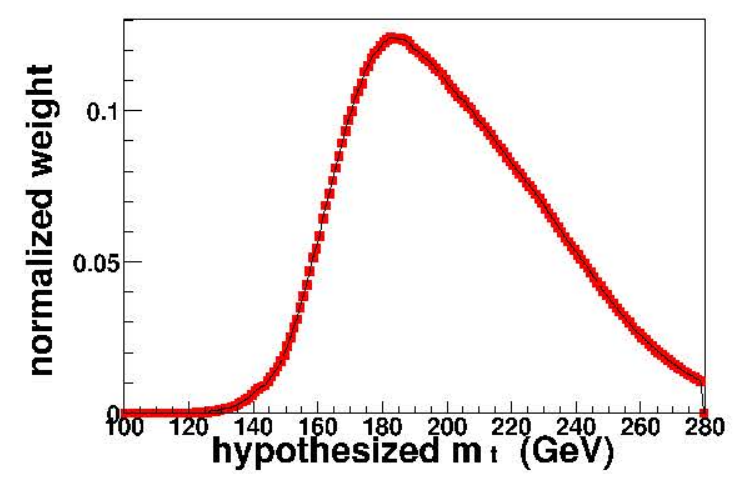

Run, Event 180326, 14448436

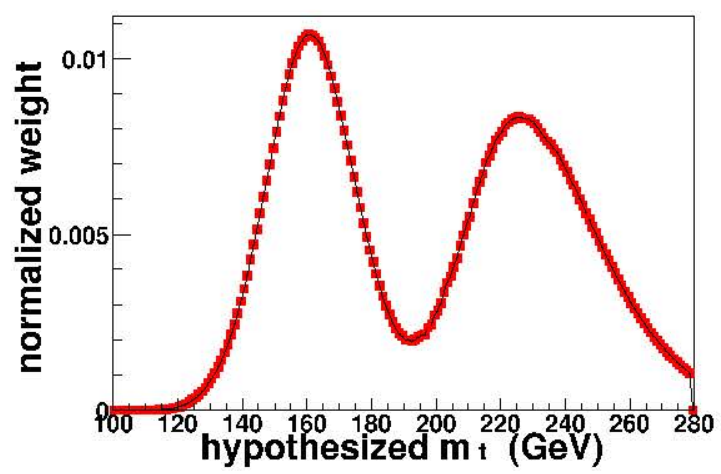

Figure L.1: Weight distributions of the candidate events in the di-clectron channcl. 

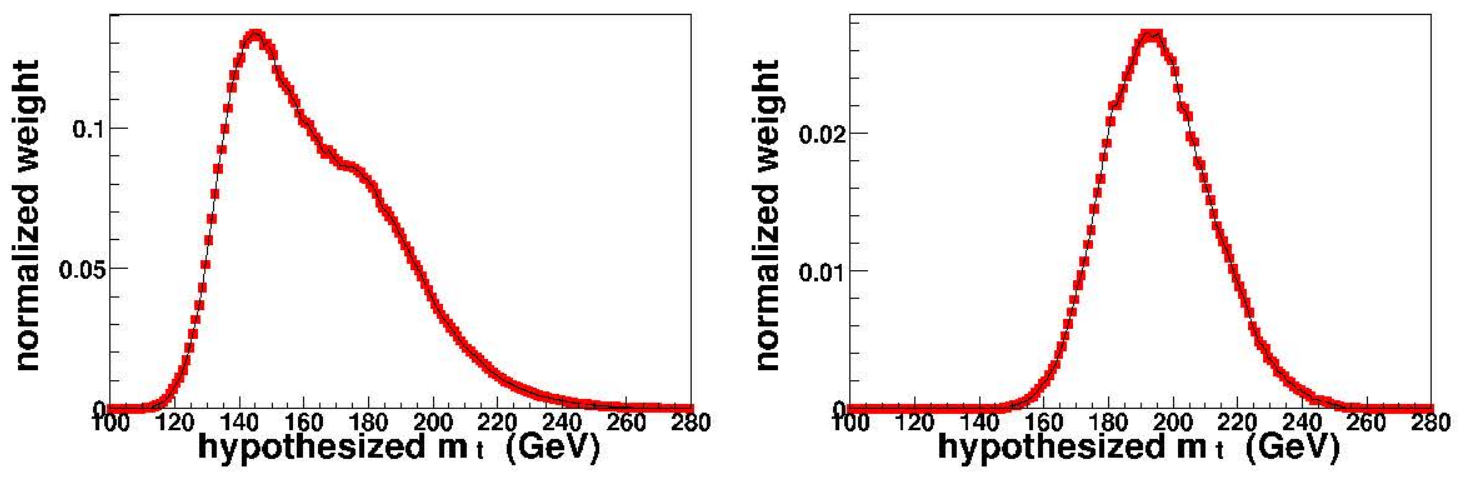

run 166779 run 121971122

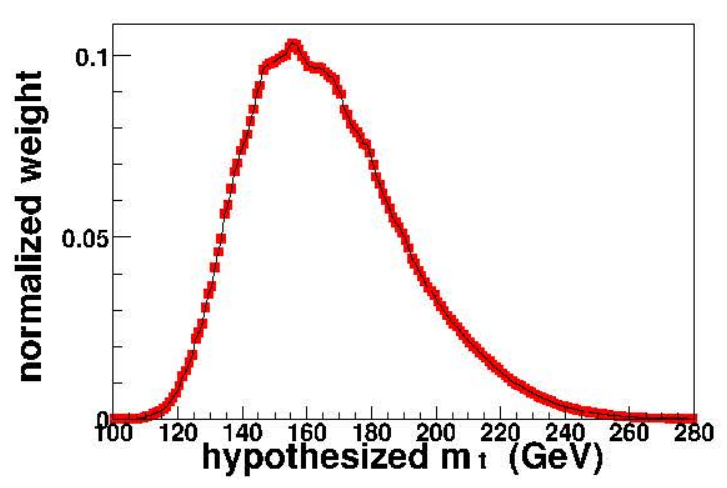

Figure 1.2: Weight distributions of the remaining candidate events selected in the di-electron channel. 
The weight distributions of the cight $e \mu$ candidate events are illustrated in the Figures L.3 and L.4.

Run, Event 168393, 1997007

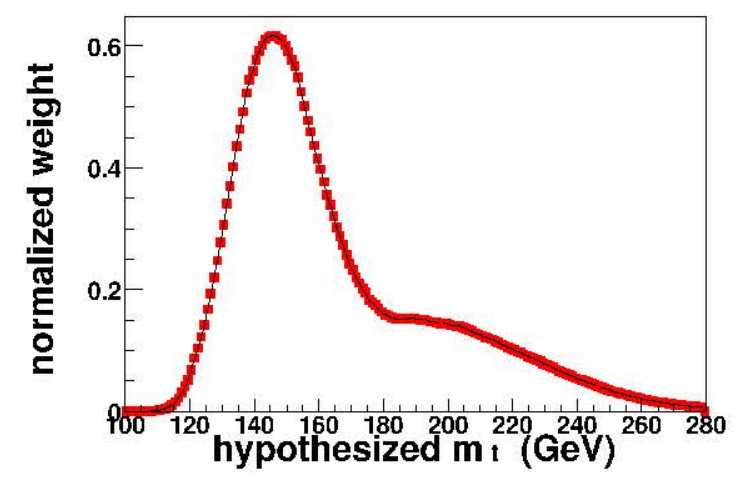

Run, Evont 177826, 15259654

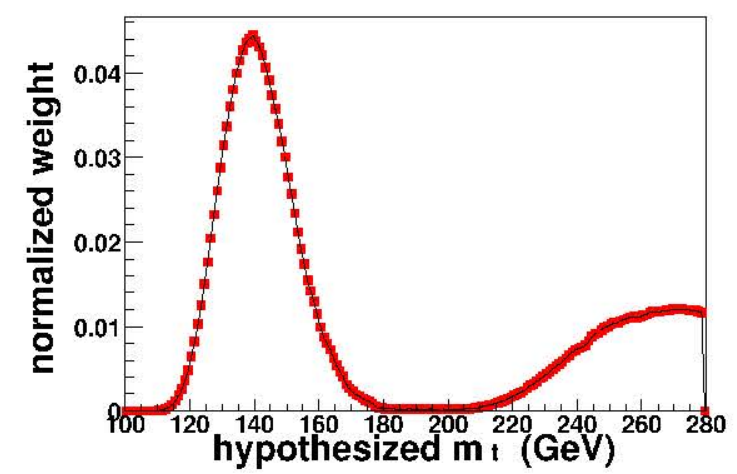

Run, Evont 174901. 8710859

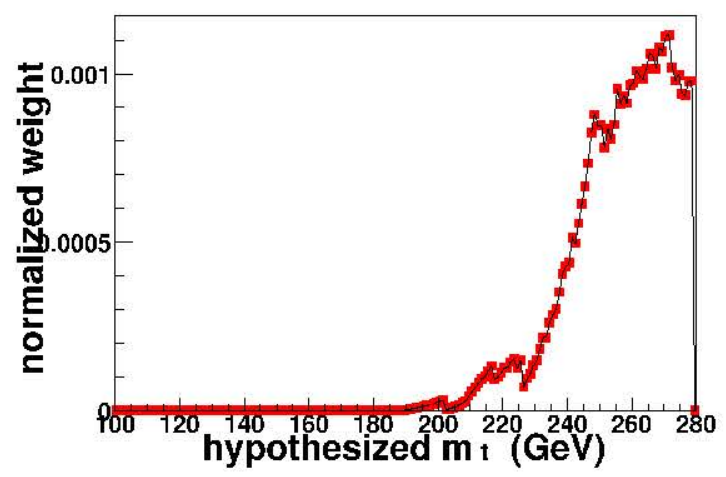

Run, Event 178159, 37315438

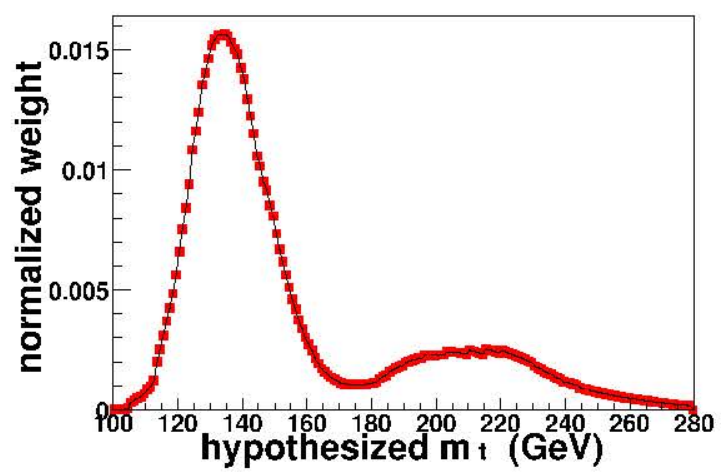

Figure L.3: Weight distributions of candidate events selected in the $e \mu$ channel. 
Run, Event 179141, 11709332

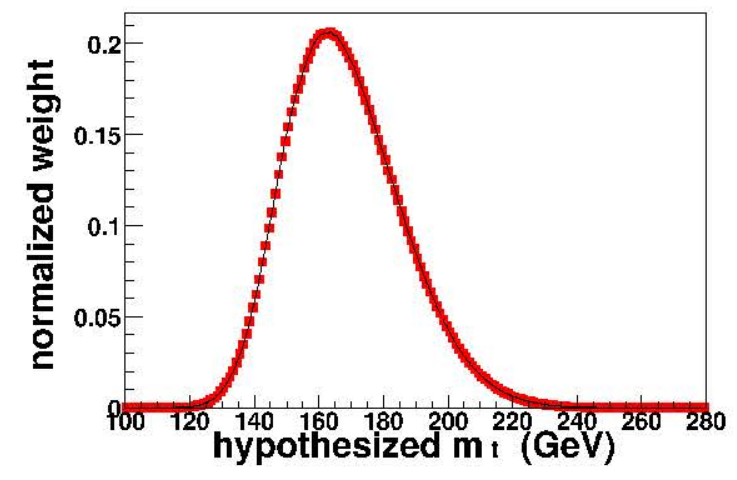

Run, Event 179195, 26386170

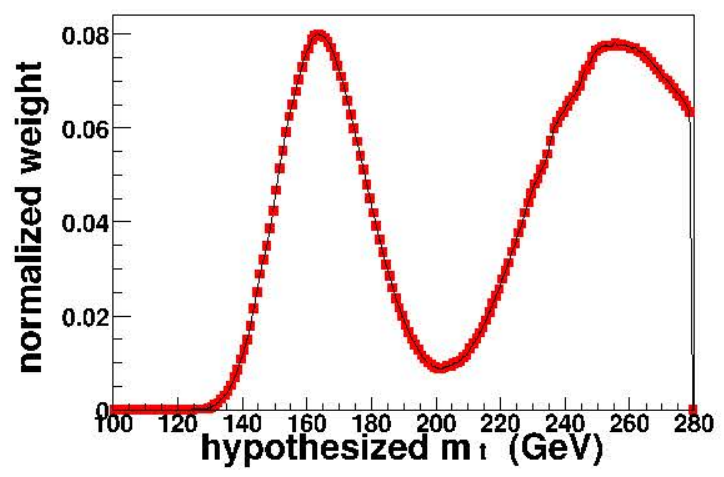

Run, Event 178733. 8735139

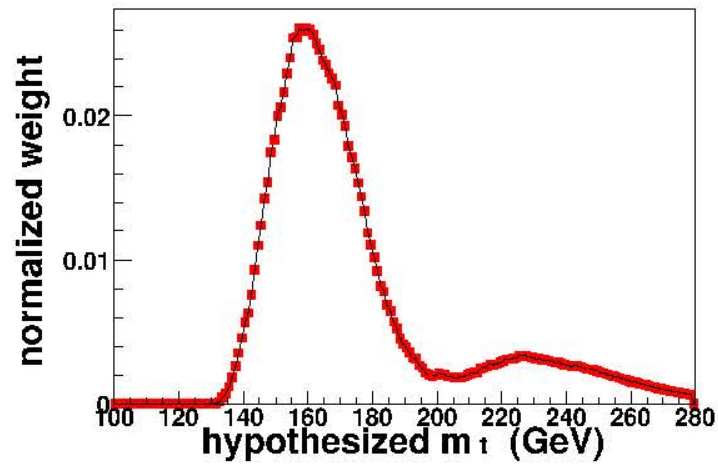

Run, Event 179331, 19617819

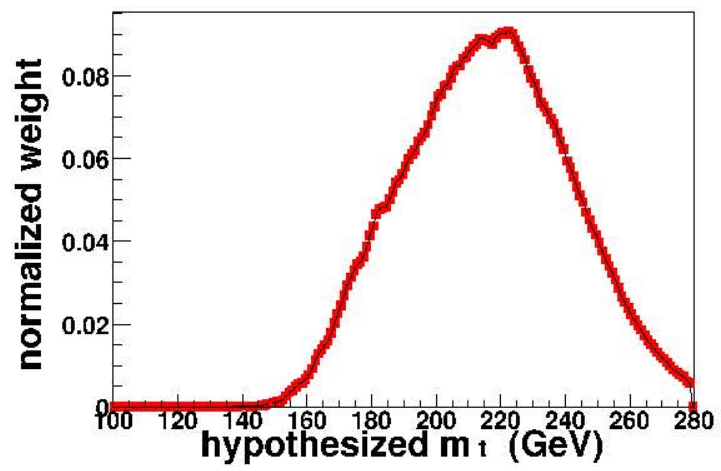

Figure L.4: Weight distributions of remaining candidate events selected in the $e \mu$ channel. 


\section{Bibliography}

[1] J. Dalton, "On the Absorption of Gases by Water and Other Liquids". Dalton read the paper before the Literary and Philosophical Society of Manchester on October 21, 1803.

"John Dalton and the Atomic Theory: The Biography of a Natural Philosopher", E. C. Patterson, Doubleday, New York, 1970. (With notes for a lecture Dalton delivered in 1810 belore the Royal Institution in London.)

Selected Classic Papers from the History of Chemistry, Carmen Giunta, Lemoyne College (1999). URL:

http://MAPLE.lemoyne.edu/ giunta/papers.html

http://web.lemoyne. edu/ giunta/dalton.html

http://web.lemoyne.edu/ giunta/dalton52.html

[2] F. Rutherford, "The Scattering of $\alpha$ and $\beta$ Particles by Matter and the Structure of the Atom", The Philosophical Magazine 21, 669 (1911).

E. Rutherford, "I'he Structure of the Atom", Nature 92, 423 (1913); The Philosophical Magazine 27, 488 (1913).

[3] ‥ Bohr, "On the Quantum Theory of Line-Spectra", Kongelige I)anske Videnskabernes Sclskabs Skrilter, Naturvidenskabelig og Mathematisk Afdeling 
(Royal Danish Acadcmy of Sciences and Letters, Papcrs, Natural Scicnces and Mathematics Section), 8 Raekke (Series), 4, \#1, pages. 1-3 (1918)

[4] H. Geiger, and F. Marsden, The Philosophical Magazine, 25, 606 (1913).

[5] J. Chadwick, and F.S. Bieler, "Collision of $\alpha$ Particles with Hydrogen Nuclei", The Philosophical Magazine 42, 923 (1921).

[6] A sense of contemporary work and discoveries can be had from: Particle Physics, One Hundred Years of Discoveries: An Annotated Chronological Bibliography. Edited by V.V. Ezhela, B.B. Filimonov, S.B. Lugovsky, B. V. Polishchuk, S. I. Striganov, Y. G. Stroganov, B. Armstrong, R. M. Barnett, D. F. Groom, P. S. Gee, T. G. Trippe, C. G. Wohl, J.D. Jackson, AIP Press and Springer publications.

[7] L. de Broglie, "Ondes et Quanta" (Waves and Quanta), Comptes Rendus 177, 507 (1923); "Quanta de Lumiere, Diffraction et Interferences" (Light Quanta, Diffraction and Interferences), Comptes Rendus (Comptes Rendus Hebdomadaires des Séances de l'Académic des Sciences) 177, 548 (1923).

[8] R. H. Kraus, Jr. "The overvicw and history of permanant magnet devices in accelerator technology", available at the URL: http://ieeexplore.ieee.org/xpl/abs_free.jsp?arNumber=305560 and references there in.

[9] S. I. Glashow, "Partial-Symmetries of Weak Interactions", Nuclear Physics 22, 579 (1961).

S. Weinberg, " $\Lambda$ Model of Leptons", Physical Revicw Letters, 19, 1264 (1967). A. Salam, "Elcmentary Particle Theory: Relativistic Groups and Analyticity" 
(Nobcl Symposium \#8), cdited by N. Svartholm, Almqvist and Wiksell, Stockholm, page 367 (1968).

[10] C. Quigg, "The State of the Standard Model", Lecture at Conference on the Physics Potential and Developement of Muon Colliders and Neutrino Factorics, San Francisco, December 15-17, 1999, available at hep-ph/0001145.

C. Quigg, "The Electroweak Theory", Flavour Physics for the Millicnium: TSAI 2000, edited by J. L. Rosner (World Scientific, Singapore, 2001), page 3-67.

R. N. Cahn, "The IIiggs Boson in the Minimal Non-Supersymmetric Standard Model", Lectrures at l'Ecole de GIF, Scptember 2001.

R. S. Chivukula, "Models of Elcetrowcak Symmetry Breaking", NATO Advanced Study Institute on Quantum Ficld Theory Perspective and Prospective, Les Houches, France, 16-26 June, 1998;

(also available at hep-ph/9803219).

C. 'T'. Hill and E. H. Simmmons, "Strong Dynamics and Electroweak Symmetry Breaking", Physics Reports (February 2002)

K. Lane, "Iechnicolor 2000", Lectures at the LNF Spring School in Nuclear, Subnuclcar and $\Lambda$ stroparticle Physics, Frascati (Romc), Italy, May 15-20, 2000; (also available at hep-ph/0007304).

K. Lane, "Two Lectures on Technicolor", available at:

hep-ph/0202255, FERMILAB-PUB-02/040-T, or BUHEP-02-15.

[11] http://www.fnal.gov/pub/inquiring/physics/discoveries/pr /top_news_release.html

CIJF Collaboration, "Observation of Top Quark Production in $p \bar{p}$ Collisions", Physical Rovicw Letters, 74, 2626 (1995). 
DØ Collaboration, "Observation of the Top Quark", Physical Revicw Letters 74, $2632(1995)$.

[12] DØ collaboration, "An Improved Measurement of the Top Quark mass", Nature, $\mathbf{4 2 9}, 638$ (2004). 1

[13] Y. Nambu, "A Systcmatics of Hadrons in Subnuclear Physics", Preludes in Theoretical Physics in IIonor of V. F. Weisskopf, edited by A. De-Shalit, II. Feshbach, and L. Van Hove, North-Holland, Amstcrdam 133 (1966).

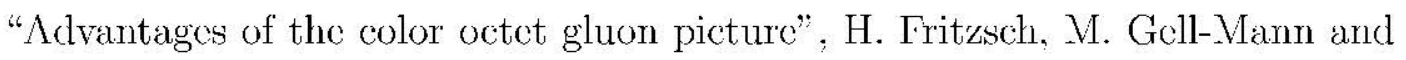
H. Leutwyler, Physics Letters, B47, 365 (1973).

"Lltraviolet Bchavior of Non-Abclian Gauge Theorics", D. Gross and F. Wilczek, Physical Review Letters, 30, 1343 (1973).

"Reliable Perturbative Results for Strong Interactions?", H. D. Politzer, Physical Review Letters, 30, 1346 (1973).

[14] P. W. Higgs, "Broken Symmetries, Massless Particles and Gauge Fields", Physics Letters 12, 132 (1964).

P. W. Higgs, "Broken Symmetries, and Masses of Gauge Bosons", Physical Review Letters 13, 508 (1964).

P. W. Higgs, "Spontancous Symmetry Brcakdown without Massless Bosons", Plyysical Review 145, 1156 (1966).

[15] S. L. Glashow, "Towards a unified theory; threads in tapestry", Review of Modcrn Physics, 52 (1980).

I. J. Aitchison and $\Lambda$. J. Hcy, Gaugc Theories in Particle Physics (Bristol: Adam Hilger 1981). 
C. Quigg, Gauge Theorics of the Strong, Weak, and Elcetromagnetic Intcractions (Addison-Wesley 1983).

M. E. Peskin, and D. V. Schrocder, An Introduction to Quantum Ficld 'Theory (Addison-Wesley 1995).

[16] Argus collaboration, "Observation of $B^{0}-B^{0}$ Mixing", Physics Letters, 192B, 245 (1987).

ALEPH collaboration, "Measurement of $B-B$ mixing at the $Z^{0}$ ", Physics Letters, 258B, 236 (1991).

[17] P. J. Franzini, "B $\bar{B}$ mixing: A review of recent progress", Physics Reports, 173, 1-62 (1989).

[18] G. L. Kane, "Top quark topics", from Gauge Bosons and Heavy Quarks: Proceedings of the Eighteenth SLAC Summer Institute on Particle Physics edited by J. Hawthome.

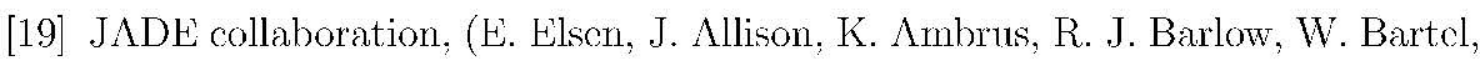
S. Bethke, et. al.), "A Measurement of the Weak Axial Couplings of the $b$ and c quark", Zcitschrift fur Physik, C46, 349-359 (1990); DESY-89-127 CELLO collaboration, (H.J. Behrend et. al.) "Heavy Quark Charge Assymetries with the CELLO Detector", Zeitschrift fur Physik, C47, 333-342 (1990). TOPA\% collaboration, "Measurements of $b$ quark forward-backward charge asymmetry and axial-vector coupling using inclusive muons in $e^{+} e^{-}$annihilation at $\sqrt{s}="$, Physics Letters, B268, 457-464 (1991).

[20] http://xxx.lanl.gov hep-cx/0104010. 
[21] K. G. Wilson, unpublished; quoted in L. Susskind, Physical Revicw Letters, 50, 1897 (1983).

G. 't Hoolt, Recent Developements in Gauge Theories, edited by G. 't Hoolt, et. al. (Plenum, New York, 1980).

P. Langacker, Electroweak model and constraints on new physics, Physics Letters B, Revicw of Particle Physics, 592 (114) (2004) and Ref \#12 thercin, Precision Tests of the Standard Electroweak Model, edited by P. Langacker.

[22] S. L. Glashow, J. Iliopoulous and L. Maiani, "Weak Interaction with LeptonHadron Symmetry", Physical Rovicw, D2, 1285 (1970).

[23] N. Kidonakis, R. Vogt, "Next-to-next-to leading order solt-gluon corrections in top quark hadronproduction", Physical Review D68 114014 (2003) http://xxx.lanl.gov hep-ph/0308222.

[21] http://lepewwg.web.cern.ch/LEPEWwG/

[25] http://tevewwg.fnal.gov/top/

[26] http://lepewwg.web.cern.ch/LEPEWWG/plots/summer/2004/

[27] DØ collaboration, "Measurement of the Top Quark Mass Using Dilepton Events", Physical Review Letters, 80, 2063 (1998).

DØ collaboration, "Mcasurement of the top quark mass in the dilepton channel", Physical Review D, 60, 052001 (1999).

[28] M.W. Gruneweald, "Experimental Tests of the Electroweak Standard Model at High Encrgics", Physics Rcports, 322, 125-316 (1999). 
[29] G. Altarelli, M.W. Gruncwald, "Precision Elcetroweak Tests of the Standard Model", CERN-PH-TH/2004-067, http://xxx.lanl.gov hep-pl//0404165

[30] The ALEPII, DELPIII, L3, OPAL, SLD Collaborations and the LEP Electrowcak Working Group, "A Combination of Prcliminary Elcctrowcak Mcasurements and Constraints on the Standard Model", http://xxx.lanl.gov hep-cx/0312023, and reforences therein.

[31] http://www. borg.umn.edu/topaz/

[32] http://xxx.lanl.gov hep-ph/9908433;

http://www-zeuthen.desy.de/ riemann/Zfitter/zf .html

[33] H. Burkhard, B. Pietrzyk, "Update of the Hadronic Contribution to the QED Vacuum Polarization", Physics Letters B 513, 16 (2001).

[34] http://www.fnal.gov

[35] http://www-do.fnal.gov

[36] http://www-bd.fnal.gov

[37] http://www-bd.fnal.gov/public/antiproton.html

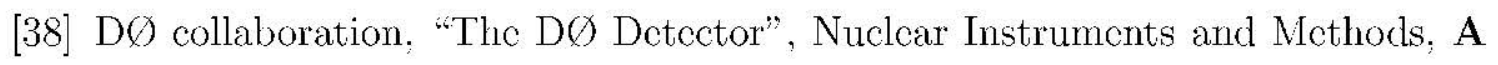
338, 185 (1994).

DØ collaboration's Run II Nuclear Instruments and Mcthods paper in preparation.

[39] R. Dower and U. Heintz, "High Energy Neutron Effects on DØ Silicon Micorstrip Detcctor Components", Intcrnal DØnote \#3761 
[10] T. Behnkc, ct. al. "Radiation Hardness and Lincarity Studics of CVD Diamonds", hep-ex/0212018 T. Bchnkc, ct. al., "Elcctromagnctic Radiation Hardness of Diamond Detectors", Nuclear Instruments and Methods A489, 230-240 (2002)

[41] http://xxx.lanl.gov, hep-ph/0403045

[42] http://www.phys.psu.edu/ cteq

[43] Alpgen http://xxx.lanl.gov, hep-ph/0206293

[44] http://www.thep.lu.se/ torbjorn/Pythia.html

[45] http://hepwww.rl.ac.uk/theroy/seymour/herwig/

[46] http://www.phy.bnl.gov/ isajet/

[17] http://ucsb.edu/people/lange/EvtGen

[18] http://wwwasd.web.cern.ch/wwwasd/geant/

[49] http://www-d0.fnal.gov/computing/MonteCarlo/MonteCarlo.html http://www-d0.fnal.gov/computing/MonteCarlo/simulation/dogstar.html

[50] http://www-d0.fnal.gov/computing/MonteCarlo/simulation/d0sim.html

[51] DØ collaboration, "Measurement of the $t \bar{t}$ Production Cross-section at $\sqrt{s}=$ $1.96 \mathrm{TeV}$ in the Di-lepton Final States", DØ Internal Note \#1421.(Moriond 2004)

[52] S. Anderson, S. Burke, S. Banerjee, B. Choudhary, C.Clement, K. Johns, R.

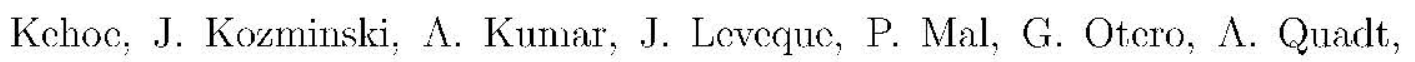


K. Ranjan, E. Shabalina, "Veasurement of the tī Production Cross-section at $\sqrt{s}=1.96 \mathrm{TeV}$ in Dilepton Final States", Internal Dø Note \#4683.

http://www-d0.fnal.gov/Run2Physics/top/private /internal_PubsReview.html version v1.5.1 (I)ecember 29, 2004)

J. Kozminsky, Ph.D. dissertation (in preparation), P. K. Mal, Ph.D. dissertation (in preparation), S. Burkc, Ph.D. disscrtation (in preparation),

[53] F. Varnes, "Track reconstruction efficiency measurement with single muons", DØ Internal Note \#4317.

[54] A. Kumar, R. Kehoe, K. Ranjan and B. C. Choudhary, "Oversmearing of Missing Transverse Encrgy in $Z \rightarrow e e+X$ Monte Carlo Events", Internal D $\varnothing$ Note \# 4551 .

[55] S. N. Fatakia and U. Heintz, "Measurement of Efficiencies of Central EM Triggers from Online Trigger Towers", Internal Dø Note \#4105.

[56] Kalman, " $\Lambda$ New $\Lambda$ pproach to Lincar Filtcring and Prediction Problems", Journal of Basic Engineering, 82 series D 35-45 (1960).

[57] M. Klute, "A Measurement of the $t \bar{t}$ Production Cross Section in Muon + Jets at $\sqrt{s}=1.96$ ' $\mathrm{lcV}^{\prime}$ ", Ph.D. disscrtation, University of Bonn, page 58, Fobruary 2004.

[58] D.Whiteson, "Muon Tracking in the Calorimeter", Internal Dø Note \#3996

[59] L. Duflot, "MC studies of jet algorithms in RUN II framework", Internal DQ Note \#3716,

L. Duflot, "Simple cone preclustering for cone jets", Internal DØ Note \#3749, 
G. Blazcy, J. R. Dittmann, S. D. Ellis, V. Danicl Elvira, K. Frame, S. Grinstcin, R. Hirosky, R. Peigaia, H. Schellman, R. Snihur, V. Sorin, D. Zeppenfeld, "Run II Jet Physics", Internal DØ Notc \#3750.

[60] A. Garcia-Bcllido, S. Lager, F. Rizatdinova, A. Schwartzman and G. Watts, "Primary Vertex certification in p14", Internal DØ Note \#4320.

[61] http://www.fnal.gov/pub/presspass/press_releases/selex_6-17.html

[62] S. J. Wimpenny, et. al., "The Hadron and Electron response of uranium/liquid argon calorimeter modules for the D-Zero detcetor", Nuclear Instruments and Methods, A279, 107 (1989).

Paolo Franzini et. al., "Performance of the DØ Lranium-liquid Argon calorimeter modules", Nuclear Instruments and Methods, A289, 438 (1990).

A. L. Spadafora, et. al., "Liquid argon calorimetry at the SSC", Nuclear Instruments and Methods, A315, 279 (1992).

[63] J. Zhu, "Detcrminnation of Electron Energy Resolution using P14 $Z \rightarrow e^{+} e-$ data", Internal DØ Note 4323.

J. Zhu1, "Direct Mcasurement of the $W$ Boson Decay Width in ProtonAntiproton Collisions at $\sqrt{s}=1.96 \mathrm{TeV}$ ", Ph.D. dissertation, University of Maryland, page 74, October 2004.

[64] http://www.pdg.lbl.gov/pdg.html

[65] ALEPH, DELPHI, L3, OPAL, LEP, Electrowcak Working group, and SLD Heavy Flavor Group, hep-ex/0212036, http://www.cern.ch/LEPEWWG/ 
[66] S. Jain, "Scalc and Over-smcaring lor MC Elcctron", Intcrnal DØ Notc \#1402.

[67] C. Clement, F. Deliot, T. Golling, K. Haganaki, B. Leonhardt, M. Mulders, E. Nurse, S. Soldner-Remboldt, J. Stark, "Muon ID certification for p14", Internal DQ Note \#4350.

[68] M. Zdrazil, "Understanding the di-muon data", Internal DØ Note \#4218.

[69] http://www-d0.fnal.gov/phys_id/jes/do_private/certified /v5.1/links.html

Older versions of the Jet Energy Scale Group's notes are:

F. Canelli, J. Coss, A. Goussiou, I lashvili, R. Kehoe, A. Kupco, S. Muanza, V. O'Dell, N. Parua, M. Petteni, A. Schwartzman, "First Jet Energy Scale at D0 in Run II (for p10 Data and Monte Carlo)" Internal DØ Note \#4110.

J. Coss, A. Goussiou, I. Iashvili, S. Muanza, V. O'Dell, N. Parua, P. Perea, A. Schwartzman, "Jet Encrgy Scalc for p11 Data", Intcrnal DØ Notc \#1112 J. Coss, S. N. Fatakia, A. Goussiou, U. Heintz, I. lashvili, M. Kopal, A. Kupco, S. Muanza, V. O’Dell, N. Parua, P. Perca, "Jct Energy Scalc and Resolution for p13 Iata and Monte Carlo", Internal I) Ø Note \#4115.

DØ collaboration, "Jet Energy Scale at DØ", (Run I) Internal DØ Note \#3287.

[70] S. N. Fatakid and U. Heint\%, "Average out-of-cone showering corrections for fixed size cone jet algorithms", Internal D $\varnothing$ Note \#4300 and references therein.

[71] S. N. Fatakia and U. Heintz, "Correcting the reconstructed jet's 4-vector to the parton level", Internal D $\varnothing$ Note \#1247, and references thercin. 
[72] R. H. Dalitz, G.R. Goldstcin, "Analysis of top-antitop production and dilepton decay events and the top quark mass", Physics Letters B, 287, 225 (1992).

[73] R. II. Dalitz, G.R. Goldstein, "Decay and polarization properties of the top quark", Physical Revicw D, 45, 1531 (1992).

[74] R. A. Fisher, "On the Mathematical Foundations of Theoretical Statistics", (1922): "Theory of Statistical Estimation", (1925);

"Inverse Probability", (1930);

"Two New Properties of Mathematical Likelihood", (1934).

the papers mentioned above are also presented on thw world wide web at the LRL:

http://www. economics.soton.ac.uk/staff/aldrich

/fisherguide/prob+lik.htm

[75] R. P. Feynman, "Space-Time approach to Quantum Elcctrodynamics", Physical Review 76, 769 (1949).

[76] K. Kondo, "Dynamical Likelihood Method for Reconstruction of Events with Missing Momentum. I. Method and Toy Models", Journal of the Physical Society of Japan 57, 4126 (1988). K. Kondo, "Dynamical Likelihood Method for Reconstruction of Events with Missing Momentum. II. Mass Spectra for $2 \rightarrow 2$ Processes", 60, 836 (1991).

[77] J. H. Kuhn, "How to measure the polarization of top quarks", Nuclcar Physics B 237, 77 (1984).

A. Ciarnecki, M. Jczabek, J. H. Kuhn, "Lepton spectra from decays of polarized top quarks", Nuclear Physics B 351, 70 (1991). 
[78] S. N. Fatakia, U. Heintz, L. Sonnenschcin, "Top Mass Measurement in the Dilepton Channel", Internal DØ Note \#4677.

[79] L. IIeintz, "Top Mass Analysis of Dilepton Events", Internal DØ Note \#2658 U. Heintz and J. Kotcher, "A Measurement of the Top Quark Mass with Dilepton Events", Internal DØ Note \#2659

[80] http://budoe.bu.edu/ sfatakia/TALKS/APS_2002.pdf

[81] http://www-d0.hef.kun.nl///fullAgenda.php?ida=a04770

[82] R. Raja, "Measuring the top quark mass usingthe dilepton decay modes", Physical Rovicw, D 55, 2902 (1997);

R. Raja, "Remarks on the error's associated with the Dalitz-Goldstein method", Physical Rovicw, D 56, 7165 (1997).

[83] R. Barlow and C. Becston, "Fitting using finite Monte Carlo samples", Computcr Physics Communications, 77, 219-228 (1993).

[84] http://www.root.cern.ch

[85] G. Cowan, Statistical Data Analysis, Oxford University Press, 1998

[86] A. G. Frodesen, O. Skjeggstad, and II. Tofhte, Probability and Statistics in Particle Physics, Universitetsforlaget (1979)

[87] D. Scott, "On optimal and data-bascd histograms", Biomctrika, 66, 605-610 (1979).

[88] A. J. Izenman, "Recent developements in non-parametric density estimation", Journal of the Amcrican Statistical Association, 84 (413), 205-224 (1991). 
[89] P. Bevington and K. D. Robinson, Data Reduction and Error Analysis for the Plysical Sciences (Mc-Graw Hill),

L. Lyons, Statistics for Nuclear and Particle Physicists; (Oxford University Press)

$[90]$ S. N. Fatakia, for the $D \varnothing$ collaboration "A method of extracting the mass of the top quark in the di-lepton channel using the DØ Detector", http: //xxx. lanl.gov hep-ex/0409013

[91] http://www-d0.fnal.gov/Run2Physics/top/private/winter04/ winter04_general_ljets_mass_note_v0_0.ps

[92] S. N. Fatakia, An update at tho Top Quark Propertics Mecting, Fobruary 25, 2004. Talk available at the URI:

http://www-d0.hef .kun.nl///fullAgenda.php?ida=a04371

[93] http://www-d0.fnal.gov/Run2Physics/WWW/results/top.htm

[94] K. Black and M. Narain, "Veasurement of the Top Mass: The Low Bias Template Method", Internal D $\varnothing$ Note\# 4464.

M. Mulders, "Top Mass Mcasurement in the Lepton+Jets Channel using the Ideogram Method in Rum II", Internal I) Ø Note\# 4429.

[95] http://www-cdf.fnal.gov/physics/new/top/top.html http://www-cdf.fnal.gov/physics/new/top/summaryplots /masssummary.txt

[96] "Future of Electroweak Physics at the Fermilab Tevatron", Report of the I'ev_2000 Study Group, edited by D. Amidei and R. Brock, Fermilab-Pub$96 / 082$. 
[97] B. Schwarzschild, "Rc-cvaluation of Top Quark Data Raises Estimate of Higgs Boson's Mass", Physics Today, 57 \#9 26-27, (August, 2004), URL: http: //www physicstoday . org

[98] R. K. Bock and A. Vasilescu, The Particle Detector BricfBook, Springer publications. Also available from the URL: http://www.cern.ch/Physics/ParticleDetector/BriefBook/

[99] http://www-do.fnal.gov/trigger/stt/

[100] http://joule.bu. edu/ hazen/sarosh/ http://ohm.bu.edu/ hazen/my_do/

[101] R. Fernow, Introduction to experimental particle physics, Cambridge Universily Press, 1986.

[102] G. F. Knoll, Radiation Detection and Mcasurcment, John Wilcy \& Sons, Incorporated, third edition (1999).

[103] 1). Green, Physics of Particle Detectors, Cambridge University Press (2000).

[104] R. Wigmans, "On the energy resolution of of uranium and other hadron calorimcters", Nuclear Instruments and Mcthods, A259, 389 (1987). R. Wigmans, Calorimetry: Fnergy Measurement in Particle Physics, Oxford University Press (2000). 


\section{SAROSH NOSHIR FATAKIA}

e-mail: sfatakia@bu.cdu

web: http://people.bu.edu/ sfatakia

\section{Education:}

M.S. Syracuse University, Syracuse, USA (1999).

Post M.Sc. Saha Institute of Nuclear Physics, Calcutta, India (1997).

M.Sc. University of Calcutta, Calcutta, India(1996).

B.Sc. University of Calcutta, Calcutta, India (1994).

\section{Fellowships:}

Department of Physics, Boston University

- Research Assistant, Spring 2000-Fall 2004.

- Teaching Fellow, Fall 1999.

Department of Physics, Syracuse University

- Research Assistant, Summer 1998-Spring 1999.

- Teaching Assistant, Summer 1999, Fall 1997 - Fall 1998.

Saha Institute of Nuclear Physics, University of Calcutta

- Research Fellow, Fall 1996-Summer 1997.

\section{Contributions to conferences}

- APS April Mceting, Albuqucrquc, NM, 2002. Conlribuled lalk.

- APS April Meeting, Denver, CO, 2004. Contributed talk.

- "Physics in Collisions 2004", Boston, MA, 2004. Poster Presentation. Proceedings of the conference available as hep-ex/0409013. 